Stratigraphic Sections of the

Phosphoria Formation

in Idaho,

1947-48, Part I

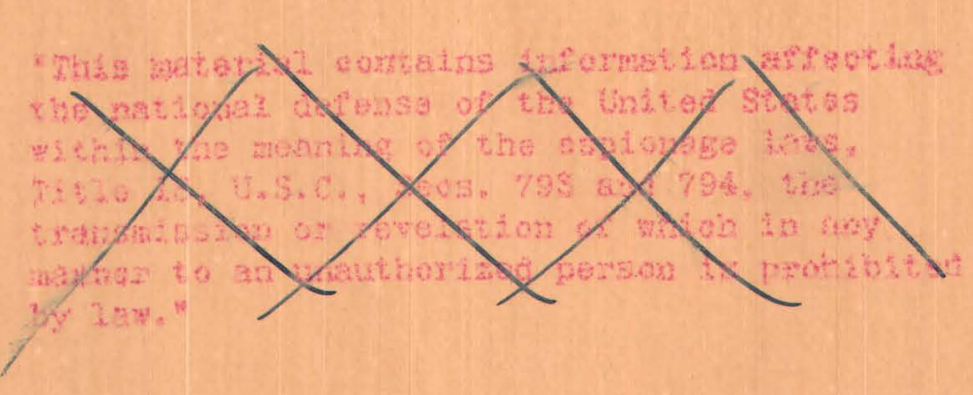

Trace Elements Investigations Report 183

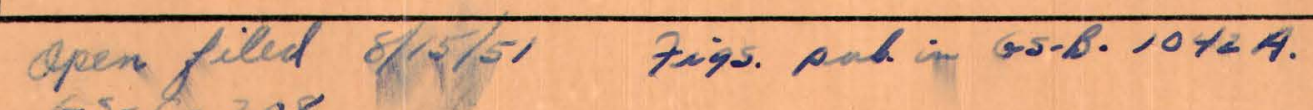

-S- -208 .

UNITED STATES DEPARTMENT OF THE INTERIOR GEOLOGICAL SURVEY 


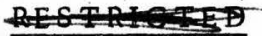

USGS - TEI Report 183

GEOLOGY - MINERALOGY

Distribution (Series A)

No. of copies

American Cyanamid Company, Winchester . . . . . . . 1

Argonne National Laboratory . . . . . . . . . . 1

Atomic Energy Commission, Washington _. . . . . . . . 1

Battelle Memorial Institute, Columbus。 . . . . . . . . . 1

Carbide and Carbon Chemicals Company, Y-12 Area . . . . . 1

Division of Raw Materials, Grants . . . . . . . . . 1

Division of Raw Materials, Denver ............ 1

Division of Raw Materials, Hot Springs, . . . . . . . . 1

Division of Raw Materials, New York 。 . 。 . . . . . 6

Division of Raw Materials, Salt Lake City _. . . . . . . 1

Division of Raw Materials, Richfield . . . . . . . . 1

Division of Raw Materials, Butte . . . . . . . . . . 1

Division of Raw Materials, Washington, . . . . . . . 3

Dow Chemical Company, Pittsburg .. . . . . . . . . 1

Exploration Division, Grand Junction Operations Office。 . . . . 1

Grand Junction Operations Office . . . . . . . . . . . . 1

Technical Information Service, Oak Ridge 。 . . . 。 . . 6

Tennessee Valley Authority, Wilson Dam. . . . . . . . 1

U。 S。 Geological Survey:

Mineral Deposits Branch, Washington . . . . . . . . 2

Geochemistry and Petrology Branch, Washington ........ 1

Geophysics Branch, Washington ............ . . 1

Alaskan Geology Branch, Washington 。 。 . 。 。 . . 1

Fuels Branch, Washington . . . . . . . . . . 1

L。 R。 Page, Denver。 . . . . . . . . . . . . 2

R。 P。 Fischer, Grand Junction 。. . . . . . . . . . 1

A。 E. Weissenborn, Spokane . . . . . . . . . . . 1

C。 B. Hunt, Plant City ............... 1

N. M. Denson, Denver 。. . . . . . . ..... 1

R。 W Swanson, Spokane 。 . . . . . . . . . . . 2

A $H_{\circ}$ Koschmann, Denver 。. . . . . . . . . . 1

E. Ho Bailey, San Francisco 。. . . . . . . ... 1

C. E. Dutton, Madison _. . . . . . . . . . . 1

$\mathrm{R}_{\circ} \mathrm{A}$ 。 Laurence, Knoxville . . . . . . . . . . . 1

$\mathrm{R}_{\text {。 J }}$ Roberts, Salt Lake City _.............. . 1

TEPCO, Washington:

Resource Compilation Section . . . . . . . . . . . . 2

Reports Processing Section . . . . . . . . . . . . 2

(Including master) 


\section{OFFliALL USE BWH \\ RDOCDOC \\ Sक्य $20 \mathrm{~N}$}

\section{Grassification changed to OFF!CIAL USE OALY \\ Geology - Mineralogy} by authority of P.L. Merritt bignature of peison making change, and date thereof

UNITED STATES DEPARTMENT OF THE INTERIOR

GEOLOGICAL SURVEY

STRATIGRAPHIC SECTIONS OF THE PHOSPHORIA FORMATION

IN IDAHO $1947-48$, PART I**

V.E. McKelvey, D。F。 Davidson, $E_{0}$, W。 O'Malley,

$L_{0} E_{0}$. Smith, $F_{0}$. $C_{0}$ Armstrong, and $R_{0} P_{0}$. Sheldon

December 1952

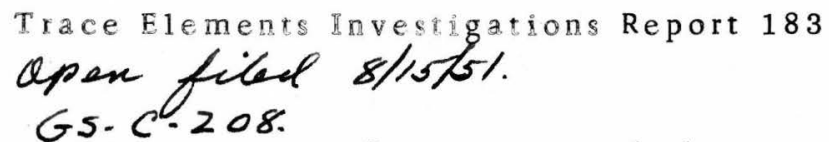

This preliminary report is qustributed in GS-B- $1042 A$. without editorial and technical review for conformity with official standards and nomenclature. It is not for public inspection or quotation.

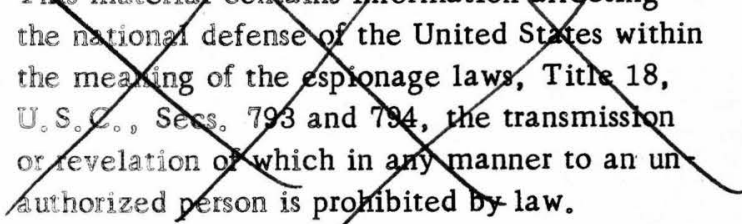

This report concerns work done on behalf of the Division of Raw Materials of the U. S. Atomic Energy Commission 


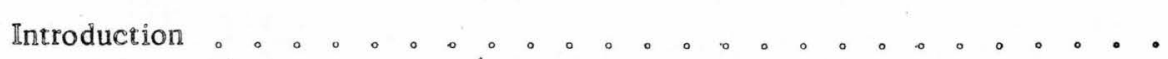

Lot no. Page

Acknowledgments

Field and laboratory procedures

Preparation of sections for description and sampling

Measurement and description of strata

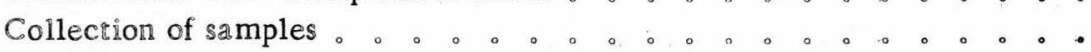

Treatment and analysis of samples $0.0,0.0 .0 .0 .0$.

Interpretation of chemical data $0^{\circ} \circ 0^{\circ} \circ 0^{\circ} \circ 0^{\circ} \circ 0^{\circ} \circ 0^{\circ} \circ 0^{-}$.

Classification and nomenclature of rocks of the Phosphoria formation . . .

Stratigraphy of the Phosphoria formation in southeastern $\mathrm{Id}$ ho 。 。 。 . .

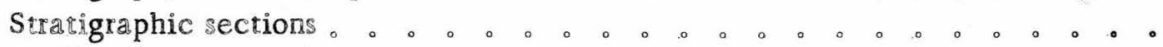

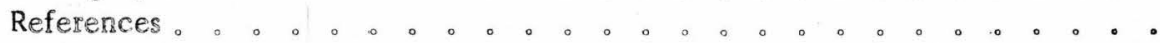

Tables of stratigraphic sections

Diamond drill hole 8, Fort Hall mine 。

Diamond drill hole 9, Fort Hall mine 。 o 。 。 。 。 。 。 。 。 128013

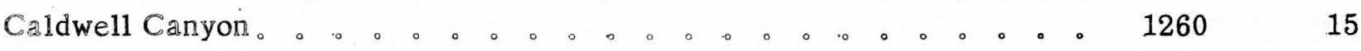

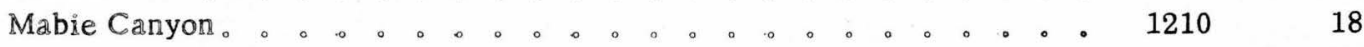

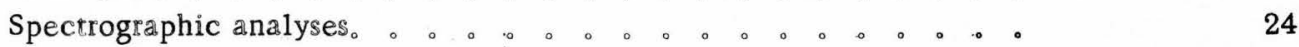

Conda 。 . . . . . . . . . . . . . . . . . . . . . . $1200 \quad 29$

Spectrographic analyses, . . . . . .......... . . . . 34

Diamond drill hole 6, Slug Creek Valley 。 。 。 。 。 。 。 . . . $1277 \quad 38$

Diamond drill hole 3, Middle Sulphur Canyon 。 。 。 . 。 。 。 . . . $1274 \quad 40$

Diamond drill hole 4, Middle Sulphur Canyon 。 。 。 。 。 。 127541

Diamond drill hole 5, South Fork of Johnson Creek 。 。 。 。 。 . 127642

North Dairy。 。 。 。 。 。 。 。 。 。 。 。 。 121244

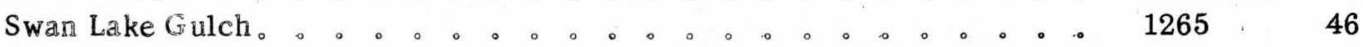

Deer Creek 。 . ० 。

IL LUSTRATIONS

Plate 1。Permian phosphate deposits of Idaho showing localities samples . . .

Figure 1. Phosphate deposits and sample localities of inset area on plate 1 .

2. Generalized typical section of Phosphoria formation in southeastern Idaho。 


\title{
STRATIGRAPHIC SECTIONS OF THE PHOSPHORIA FORMATION
}

\author{
IN IDAHO, 1947-48, PART I
}

\section{INTRODUCTION}

The Permian Phosphoria formation of the western states contains one of the world's largest reserves of phosphate. Although previous investigations (see especially Mansfield, 1927), including reconnaissance geologic mapping and sampling, established the location of most of the important deposits and their quality at scattered localities, they were not sufficiently detailed to permit a comparison of the merits of individual deposits or an appraisal of the reserves of phosphate rock that might be available under present economic conditions. Because the growing importance of the western phosphate deposits requires a better, more detailed understanding of their distribution and quality, the Geological Survey began in 1947 a comprehensive investigation, including (1) reconnaissance geologic mapping, mostly in Montana, of areas in which the Phosphoria formation could occur but where it had not previously been looked for or found; (2) geologic mapping, mostly in Montana, at a scale no smaller than $1: 62,500$, of several areas known to contain the Phosphoria formation but not previously mapped except in reconnaissance fashion; (3) geologic and topographic mapping, at a scale of $1: 12,000$, of some of the richest, thickest, and most accessible deposits; (4) measuring, describing, and sampling all beds of the phosphatic and shaly parts, and in some places the full thickness, of the Phosphoria formation and its stratigraphic equivalents at one or two localities per township over the entire field; (5) chemical and spectrographic analysis of the samples for phosphate, fluorine, minor metals, oil, and rock-forming constituents; and (6) petrologic and geochemical studies of the rocks and minerals of the formation.

These studies are designed to define the regional and local geologic structures in which the phosphate beds lie, to provide a basis for the estimation of reserves of the inferred class over the entire region, and to determine the origin of the rocks and the elements contained in them. The data collected are not of the detail required to plan actual mining operations, but it is hoped they will guide industry in the selection of individual deposits worthy of further exploration.

Most of the field work contemplated as a part of this investigation is now completed. Although the data will not be compiled or published in final form for some years to come, segments of the data, accompanied by little or no interpretation, will be published as preliminary maps or reports as they are assembled. The present report is the first of a series presenting in abbreviated form the descriptions and analyses of the beds measured and sampled at various localities in southeastern Idaho (pl. 1). Companion reports presenting segments of the data from Montana, Wyoming, and Utah (Swanson and others, 1952, and McKelvey and others, $1952 \mathrm{a}$ and $1952 \mathrm{~b}$ ) are being released at the same time as this report, and others are in preparation.
A large number of people have taken part in this investigation. In addition to the authors, R. M. Campbell, R. A. Gulbrandsen, R. A. Hoppin, D. M. Larrabee, O. A. Payne, R. S. Sears, R. A. Smart, R. G. Waring, and R. A. Weeks participated in the description of the strata and collection of the samples referred to in this report. D. B. Dimick, Jack George, W. S. Hunziker, J. E. Jones, H. A. Larsen, and T. K. Rigby assisted in the preparation of trenches and collection, crushing, and splitting of samples in the field. The diamond drilling described in this report was undertaken for the Geological Survey as a cooperative project by the U. S. Bureau of Mines. This drilling was under the supervision of A. E. Long (1949).

The laboratory preparation of samples for chemical analysis was done under the direction of W. P. Huleatt (1950), who also designed a crushing and splitting apparatus for field use. The analyses for $\mathrm{P}_{2} \mathrm{O}_{5}$ and acid insoluble and most of those for $\mathrm{Al}_{2} \mathrm{O}_{3}, \mathrm{Fe}_{2} \mathrm{O}_{3}$ Bureau of Mines at the Northwest Electrodevelopment Laboratory, Albany, Oreg., under the direction of S. M. Shelton and M. L. Wright. The spectrographic analyses in the Bureau of Mines Laboratory were prepared by D. M. Mortimer. Analytical work by the Geochemistry and Petrology Branch of the Geological Survey has been done under the direction of J. C. Rabbitt by chemists I. Barlow, A. Caemmerer, J. Greene, N. Guttag, and E. H. Humphrey.

Compilation of the data has been largely by R. P. Sheldon and F. D. Frieske under the supervision of R. W. Swanson. Organization of the tabular data has been largely by Anita Cozzetto.

\section{Acknowledgments}

Special thanks are due W. W. Rubey, J. S. Williams, and A. E. Weissenborn who have given much advice in planning and organizing the field program.

The cost of both the field and laboratory investigations has been partly borne by the Division of Raw Materials of the Atomic Energy Commission. This support is gratefully acknowledged.

It is a pleasure to acknowledge the fine cooperation extended to the field party by the local residents, property owners, and operating phosphate companies, who furnished information and services and gave access to property. A. J. Winters, Superintendent of the Montpelier schools; E. M. Norris, C. T. Russell, and L. E. Traeger of the Anaconda Copper Mining Company; D. L. King of the San Francisco Chemical Company; and G. A. McHugh and H. B. Fowler of the Simplot Fertilizer Company have been especially helpful in this connection. 


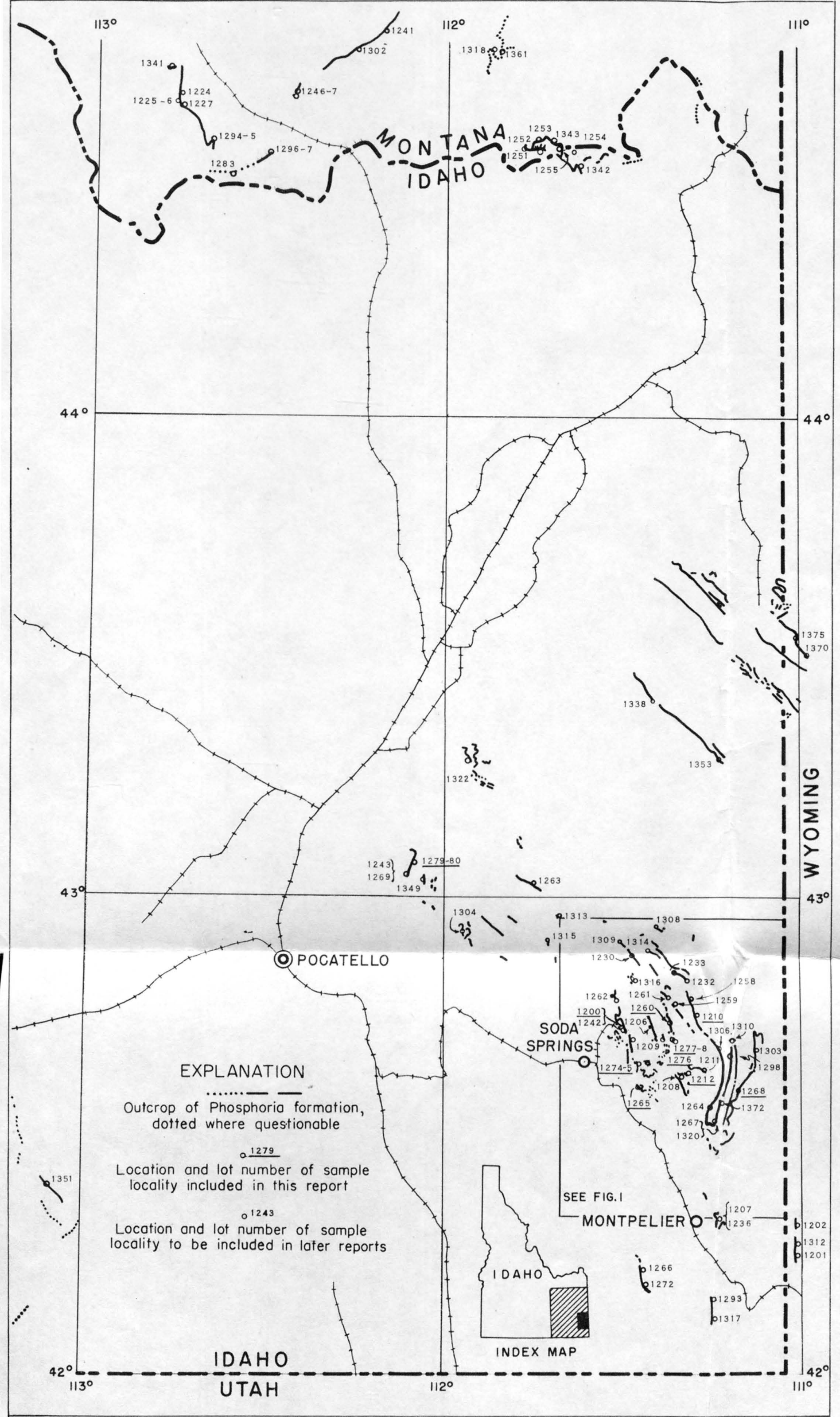

PERMIAN PHOSPHATE DEPOSITS OF IDAHO SHOWING LOCALITIES SAMPLED 


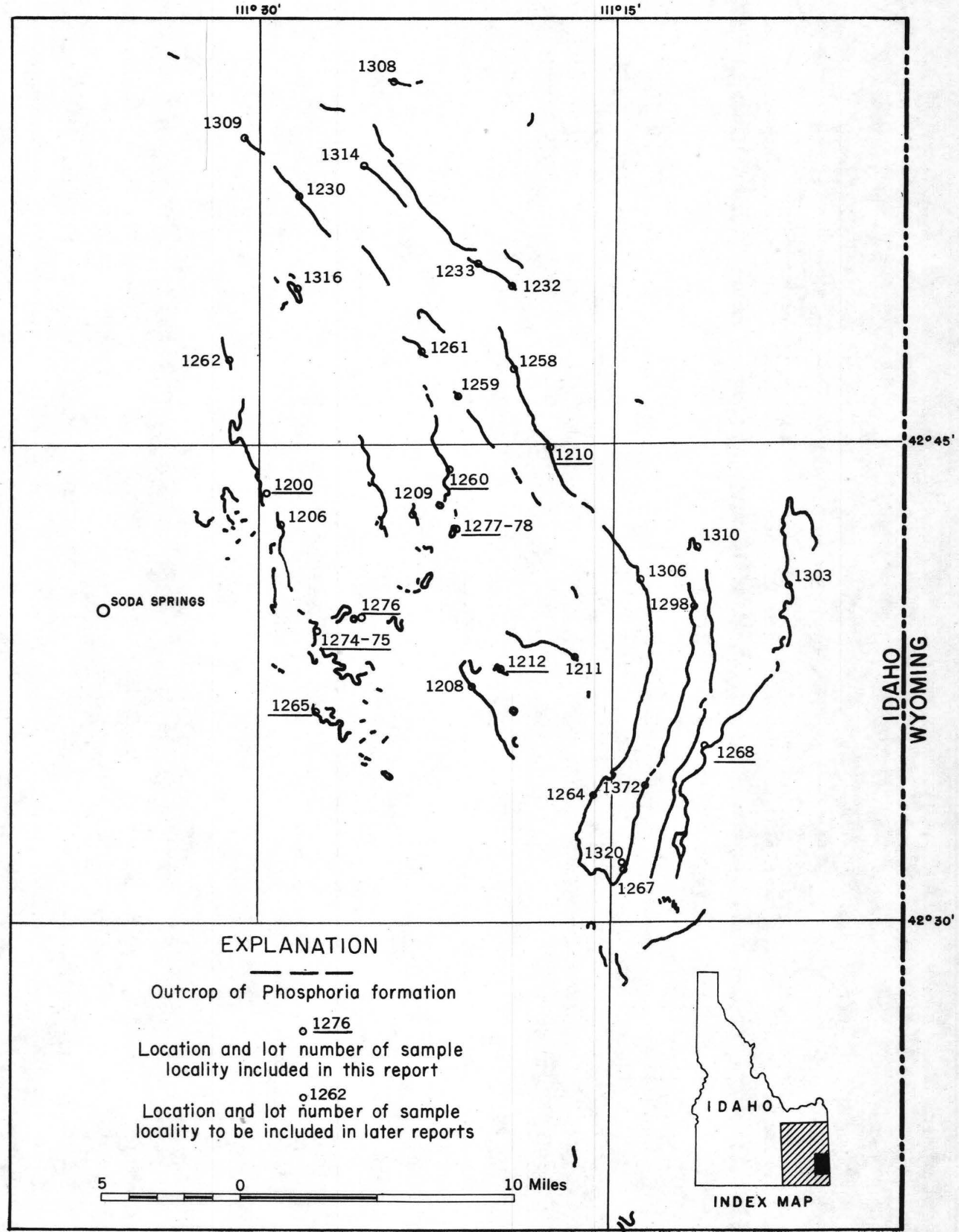

Figure 1.--Phosphate deposits and sample localities of inset area on plate 1 


\section{FIELD AND LABORATORY PROCEDURES}

The field and laboratory procedures followed in these investigations and the definitions of the lithologic terms adopted throughout the work, particularly insofar as they bear upon the interpretation of the data presented, are described in some detail in this report but will be omitted or only summarized in subsequent ones.

\section{Preparation of Sections for Description and Sampling}

Natural exposures of the phosphatic shale member of the Phosphoria formation are rare. Most of the sections measured, therefore, have been exposed artificially, generally by bulldozer but in some places by hand-trenching. The trenches, which range from 5 to as much as 30 feet in depth and from 200 to 500 feet in length, have been located mostly on steep slopes containing a minimum of foreign talus and abundant fragments of phosphate rock and in areas where the regularity of outcrops of adjacent formations and the covered interval between them indicated little or no structural complication. Despite these precautions, part if not much of the phosphatic shale proved too deeply buried to expose at several localities; the rocks exposed at most localities are weathered and in some places physically distorted or displaced by creep; and at some localities parts of the section are repeated or omitted by faults not recognizable at the surface. For these reasons the thicknesses measured do not everywhere represent the true thickness of the rocks, and the samples collected and analyzed are not wholly representative of the rocks at depth.

A few sections were obtained by diamond drilling undertaken by the Bureau of Mines in cooperation with the Geological Survey in an effort to develop a means of coring the Phosphoria formation. The engineering results of this experimental drilling have been described in another report (Long, 1949).

\section{Measurement and Description of Strata}

The full thickness, or such of it as could be exposed, of the phosphatic member of the Phosphoria formation has been described and sampled at each locality. In addition the entire formation has been described, and in some places sampled as well, at selected localities to provide information on other constituents besides the phosphate and to provide stratigraphic information for correlation purposes.

Each lithologic unit greater than 0.5 foot in thickness has been described and sampled separately (a few thinner units have been described and sampled as well), and units greater than 5 feet in thickness have been divided into two or more parts, except in nonphosphatic parts of the formation where some thicker units have not been subdivided. For identification purposes, each locality has been assigned a lot number, each bed described a bed number, and each bed sampled a sample number.

Because many people have participated in the measurement and description of the beds, it has been necessary to standardize the terminology used in the work in order to achieve uniform results. This has been achieved mainly through the use of prepared forms or tables with spaces in which to record for each unit its bed number, sample number field name, thickness, texture, grain size, hardness, thickness of bedding, presence of fossils, mineralogy, reaction to hydrochloric acid, color, jointing, and nature of contact with the underlying unit. In order that field descriptions could be augmented by laboratory petrographic studies, one or more chip samples have been collected from each unit. Only a brief abstract of this field description-bed and sample numbers, rock name, and bed thickness- is presented here, but most of the remaining data will be presented later.

\section{Collection of Samples}

Channel or bench samples have been collected from each unit over the full thickness of the phosphatic member of the Phosphoria formation and, in a few places, over the entire formation. The minimum weight of the samples is 12 pounds, but the average weight of those collected from the sections described in this report is about 35 pounds.

\section{Treatment and Analysis of Samples}

All samples have been crushed to minus $\frac{1}{2}$-inch mesh and reduced to 10 pounds in a field laboratory established at Montpelier, Idaho, using a combination jaw crusher and Vezin-type splitter designed by W. P. Huleatt (1950). The crushed samples have been split into two parts at the Geological Survey samplepreparation laboratory in Denver-one stored with no further treatment and the other ground to minus 20mesh. From the latter, two 4-ounce splits have been cut and ground to minus 80-mesh for chemical analysis. Powders for spectrographic analysis also have been prepared in the Survey's Denver laboratory.

All samples have been analyzed for $\mathrm{P}_{2} \mathrm{O}_{5}$ and acid insoluble by methods essentially the same as those described by Hoffman and Lundell (1938). In addition all samples from a few localities have been analyzed for $\mathrm{Al}_{2} \mathrm{O}_{3}, \mathrm{Fe}_{2} \mathrm{O}_{3}, \mathrm{~V}_{2} \mathrm{O}_{5}$, and loss on ignition, and semiquantitatively spectrographed for $\mathrm{Al}, \mathrm{Ba}, \mathrm{B}$, $\mathrm{Ca}, \mathrm{Cr}, \mathrm{Co}, \mathrm{Cu}, \mathrm{Fe}, \mathrm{Pb}, \mathrm{Mg}, \mathrm{Mn}, \mathrm{Mo}, \mathrm{Ni}, \mathrm{Si}, \mathrm{Ag}$, $\mathrm{Na}, \mathrm{Sr}, \mathrm{Ti}, \mathrm{V}, \mathrm{Zn}$, and $\mathrm{Zr}$ and, in a large percentage of the samples, for nearly 20 additional elements including $\mathrm{Sb}, \mathrm{As}, \mathrm{Be}, \mathrm{Bi}, \mathrm{Cd}, \mathrm{Cb}, \mathrm{Ga}, \mathrm{Ge}, \mathrm{Au}, \mathrm{In}, \mathrm{Li}$, $\mathrm{Hg}, \mathrm{Pt}, \mathrm{Ta}, \mathrm{Sn}$, and $\mathrm{W}$. Most samples representing a thickness of more than 3 feet and a $\mathrm{P}_{2} \mathrm{O}_{5}$ content in excess of 30 percent or a thickness of more than 10 feet and a $\mathrm{P}_{2} \mathrm{O}_{5}$ content in excess of 20 percent have been analyzed for $\mathrm{Al}_{2} \mathrm{O}_{3}, \mathrm{Fe}_{2} \mathrm{O}_{3}$, and loss on ignition, and most of these same samples have been spectrographed for the constituents listed above. Some of these samples have also been analyzed for $\mathrm{F}$ or $\mathrm{V}_{2} \mathrm{O}_{5}$.

A small percentage of the analyses of the samples reported here are not yet available. They will be presented in later reports.

\section{INTERPRETATION OF CHEMICAL DATA}

Nearly all the phosphate is in carbonatefluorapatite, which has a composition of about $9 \mathrm{CaO} .3 \mathrm{P}_{2} \mathrm{O}_{5} \cdot \mathrm{CaF}_{2} \cdot \mathrm{CO}_{2} \cdot \mathrm{H}_{2} \mathrm{O}$. The percentage of $\mathrm{P}_{2} \mathrm{O}_{5}$ multiplied by 2.56 , therefore, gives the approximate percentage of the phosphate minera. The percentage of tricalcium phosphate, $3 \mathrm{CaO} . \mathrm{P}_{2} \mathrm{O}_{5}$, or bone phosphate of lime (B.P.L.) as it is called in the phosphate industry, may be calculated by multiplying the percentage of $\mathrm{P}_{2} \mathrm{O}_{5}$ by 2.18 .

Acid-insoluble content may be determired by a variety of procedures that yield widely different results. In the analyses reported here, the acid-insoluble content represents that portion of the rock not soluble in aqua regia and not ignitable or volatile at about $1000^{\circ} \mathrm{C}$. The acid-insoluble fraction consists principally of silica, but it generally also includes 10-30 percent of the total iron oxide, alumina, and titania and a generally negligible percentage of phosphate, magnesia, lime, and several minor metals such as zirconium. Mineralogically it may be taken as an approximate index of the total amount of detrital minerals in the rock; the fact that some of the detritals may have been partially dissolved by the acid treatment is counterbalanced by the fact that many rocks contain some secondary chert. Attention is called to 
the fact that the acid-insoluble residue determined in this fashion includes little if any carbonaceous matter.

That portion of the rock not in carbonatefluorapatite or acid insoluble, that is, A. I. $+\left(\mathrm{P}_{2} \mathrm{O}_{5} \mathbf{x}\right.$ 2. 56), includes the $\mathrm{CaO}$ and $\mathrm{CO}_{2}$ not in fluorapatite as well as nearly all the $\mathrm{MgO}, \mathrm{Na}{ }_{2} \mathrm{O}, \mathrm{K}_{2} \mathrm{O}, \mathrm{SO}_{3}$, organic matter, and minor metals such as vanadium, chromium, nickel, zinc, etc. (even some of the zirconium is in acid-soluble form); it also includes all the $\mathrm{Fe}_{2} \mathrm{O}_{3}$, $\mathrm{Al}_{2} \mathrm{O}_{3}$, and $\mathrm{TiO}_{2}$ that would be reported in separate analy'ses for these constituents (only soluble iron, alumina, and titania are generally reported). Mineralogically this portion of the rock consits mainly of calcite and dolomite; pyrite, marcasite, and other iron sulfides; limonite, hematite, and other iron oxides; iron sulfates and gypsum; and organic matter. The minerals containing the soluble alumina, alkalis, and minor metals are as yet poorly defined, but they seem to be mainly of the hydromica type.

Loss on ignition includes chiefly carbonate $\mathrm{CO}_{2}$ organic matter, and water. It may also include some sulfur, depending upon the minerals present in the rock and the temperature reached during ignition. Loss on ignition may be taken as a rough measure of the organic-matter content if the rock contains no carbonates and little or no clay or if $\mathrm{CO}_{2}$ and water have been determined and can be subtracted from the loss on ignition.

An accurate total of all constituents is extremely difficult to obtain, even when all the constituents present, and this may include 30 or 40 , are determined. This partly because the accurate determination of many constituents in phosphate rock is extremely difficult and partly because the state of oxidation of many of the elements is not known. Thus, iron is generally reported as $\mathrm{Fe}_{2} \mathrm{O}_{3}$ but it may actually be present as $\mathrm{FeO}, \mathrm{FeS}_{2}$, etc. ; vanadium is reported as $\mathrm{V}_{2} \mathrm{O}_{5}$ but may be present as $\mathrm{v}_{2} \mathrm{O}_{3}$; and sulfur is reported as $\mathrm{SO}_{3}$ but is known to be present as organic, sulfide, and sulfate compounds. The same uncertainty applies to several other elements, particularly metals.

Because nearly all stratigraphic sections were measured and sampled at or near the surface, the analyses reported here are of partly weathered rocks. Aside from physical disintegration, the principal effects of weathering of Phosphoria formation rocks are progressive oxidation of organic matter and leaching of more soluble constituents.

In advanced stages of weathering, such as are found where the rocks immediately underlie the weathering surface developed prior to the deposition of the Tertiary Wasatch formation, the organic matter is so completely oxidized and removed that the rocks are white, light gray, or pink in color. Relatively soluble constituents, including calcium carbonate and minor metals like vanadium, are leached out, so that only the more insoluble constituents remain.

In less-advanced stages of weathering, such as those characteristic of the present weathering cycle and of most of the rocks described here, the rocks are medium to dark shades of brown or gray and some of the more soluble materials mentioned above have been leached out. Judging from the rocks exposed on various levels in the Anaconda Copper Mining Company mine at Conda, the $\mathrm{P}_{2} \mathrm{O}_{5}$ content of such moderately weathered rocks may be one or two percentage points higher than in unweathered rocks at depth.

\section{CLASSIFICATION AND NOMENCLATURE OF ROCKS OF THE PHOSPHORIA FORMATION}

Rocks of the Phosphoria and adjacent formations consist of mixtures of three types of materials: (1) detrital silicates-principally quartz, clay, mica, and feldspar; (2) chemical precipitates-dominantly the isotropic carbonate-fluorapatite (collophane), calcite, dolomite, and chert but locally including minor amounts of pyrite or marcasite, gypsum, and glauconite; and (3) carbonaceous matter. Most of the detrital materials are silt or clay size in southeastern Idaho, but sand-size particles occur in other parts of the field. Many of the chemical precipitates, particularly the phosphates, are aggregated into pellets or nodules which give a coarse-textured appearance. Because the rocks are mixtures and because they were deposited under similar physical conditions, they are remarkably uniform in appearance and they are difficult to differentiate, especially in the field. For this reason, the majority of the names applied to the rocks in the field have been revised or modified on receipt of chemical or petrographic analyses.

The most significant property of the rocks is their composition, and this has been used as the principal basis in naming them. As the rocks are mixtures, their composition seldom can be described by the use of a noun alone. The rock names reported here, therefore, generally consist of a noun, chosen to represent the dominant constituent, supplemented by adjectives representing each constituent that makes up more than 20 percent of the rock, listed in order of decreasing abundance.

Nouns describing nonsilicate rocks include phosphate rock, limestone, and dolomite. The choice of these names is not influenced by texture, whether colloform, crystalline, or fragmental. Silicate rocks, that is, those rocks that contain a predominance, either individually or collectively, of silicate minerals such as chert, quartz, clay, mica, and feldspar, are subdivided into sandstone or mudstone, depending upon whether the larger percentage of these materials is sand size $(1 / 16-2 \mathrm{~mm})$ or is finer-grained, or into chert. Because it is seldom possible to differentiate silt-size from clay-size particles in the field, and frequently not possible to do it in thin sections, in carbonaceous rocks such as these, the term mudstone has been used to include all detrital rocks containing particles smaller than sand size. Quartzite has been used to describe a sandstone so well cemented that the rock breaks through, rather than around, most of the grains.

Derivatives of all these nouns are used as adjectives_phosphatic, calcareous, dolomitic, cherty, sandy, argillaceous (silt and clay not differentiated)if the respective constituents make up 20 percent or more of the rock. Other constituents that generally do not make up 20 percent or more of the rocks, such as gypsum, glauconite, limonite, and fluorite, are mentioned, if they seem unusually abundant, by preceding the constituent name with "contains". Because nearly all rocks of the phosphatic shale member contain carbonaceous matter, though rarely in excess of or even approaching 20 percent, this constituent is generally not recognized in the rock name.

Attention should be called to the fact that certain rock names-shale (which, where rigorously defined, is a fine-grained fissile detrital rock), oolite, and pisolite-are not applied here to rocks of the Phosphoria formation. These names, which are based upon structural features, have been abandoned with some misgivings, for they are easy to use in the field. 


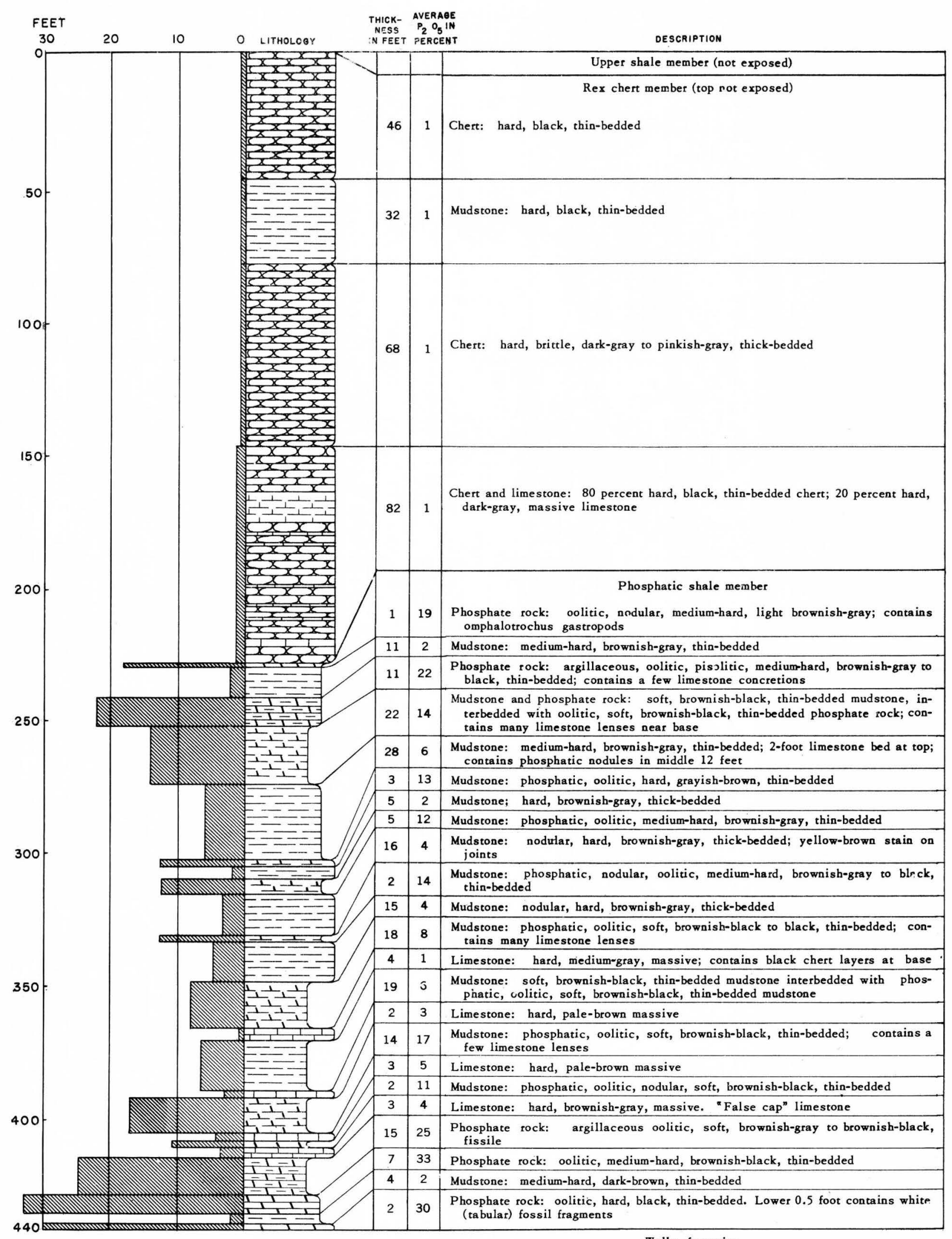

Wells formation

Figure 2.--Generalized typical section of Phosphoria formation in southeastern Idaho 
Though the term shale is discarded as a rock name, it will continue to be used as a formation name, as the "phosphatic shale member". The usage of the term in this sense for a group of fine-grained thin-bedded, but not necessarily fissile, detrital rocks is so firmly entrenched in stratigraphic nomenclature that it would be useless to try to displace it, even if it were desirable to do so.

\section{STRATIGRAPHY OF THE PHOSPHORIA FORMATION IN SOUTHEASTERN IDAHO}

At its type locality in southeastern Idaho (Richards and Mansfield, 1912), the Phosphoria formation consists of a lower member, the phosphatic shale, about 180 feet thick and an upper member, the Rex chert, about 240 feet thick; another member, a thin-bedded cherty mudstone 15 to 75 feet thick, overlies the Rex chert in most of southeastern Idaho and western Wyoming, though it is not well defined at the type locality.

The Phosphoria formation overlies the Pennsylvanian Wells formation and underlies the Triassic Dinwoody formation. The upper 50 to 75 feet of the Wells formation consists of gray fossiliferous cherty limestone that contains some thin phosphatic layers. It may be the correlative of the lowermost member ( $A$ member) of the Phosphoria formation in Montana and the lower limestone member of the Park City formation in Utah (McKelvey, 1949).

In southeastern Idaho most of the phosphatic beds are in the phosphatic shale member, and it is on this member that most of our studies have been focused. It consists of many thin layers, some of which persist over the whole area. They may be grouped into several broad units, as yet unnamed, as shown in figure 1.

\section{STRATIGRAPHIC SECTIONS}

Abstracts of stratigraphic sections measured at 12 localities, and the available analytical data, are presented in the following pages. Their locations, as well as the locations of others to be reported later, are shown in plate 1 .

The semiquantitative spectrographic analyses are based upon comparisons with a standard plate representing known quantities of the elements tested for and made at the same exposure. Greater sensitivities for many elements can be obtained by additional exposures. The standard sensitivites for the elements noted in this report are as follows:

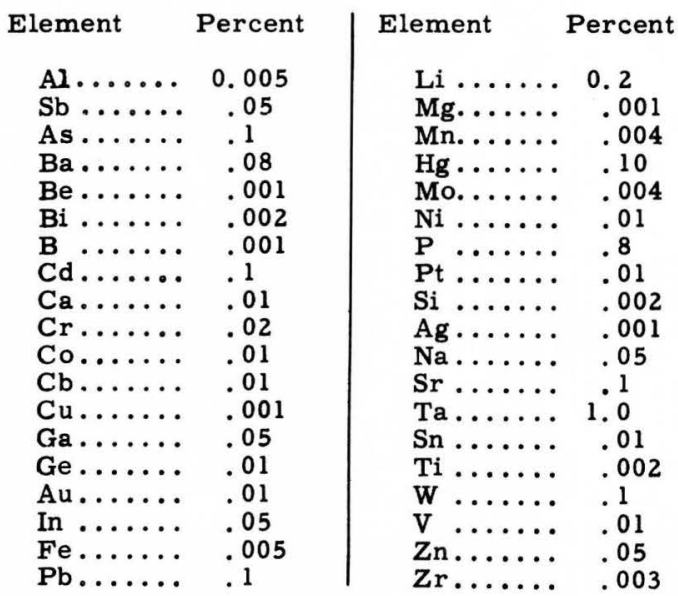

\section{REFERENCES}

Clabaugh, P. S., 1946, Permian phosphate deposits of Montana, Idaho, Wyoming, and Utah: U. S. Geol. Survey, Strategic Minerals Investigations Preliminary Map 3-198.

Hoffman, James J., and Lundell, G. E., 1938, Analysis of phosphate rock: U. S. Bur. Standards Research Paper 1095.

Huleatt, William P., 1950, Automatic sample preparation saves time, money for U.S.G.S.: Eng. and Min. Jour., vol. 151, pp. 62-67.

Long, A. E., 1949, Experimental diamond core-drilling in the Phosphoria formation in southeastern Idaho: U. S. Bur. Mines Rept. Inv. 4597.

Mansfield, G. R., 1927, Geography, geology, and mineral resources of part of southeastern Idaho: U. S. Geol. Survey Prof. Paper 152. References to other areas are listed by Clabaugh, 1946.

McKelvey, V. E., 1949, Geological studies of the western phosphate field: Am. Inst. Min. Met. Eng. Mining Trans., vol. 184, pp. 270-479.

McKelvey, V. E., Smith, L. E., Hoppin, R. A., and Armstrong, F. C., 1952a; Stratigraphic sections of the Phosphoria formation in Wyoming, 1947-48: U. S. Geol. Survey Gireular 210 . TE 184

McKelvey, V. E., Smith, L. E., Kinney, D. M. Huddle, J. W., Hosford, G. F., Sears, R. S. Sprouse, D. P., and Stewart, M. D., 1952b, Stratigraphic sections of the Phosphoria formation in Utah, 1947-48: U. S. Geol. Survey Giroular 2t1TE/-18

Richards, R. W., and Mansfield, G. R., 1912, The Bannock overthrust: a major fault in southeastern Idaho and northeastern Utah: Jour. Geology, vol. 20 , p. 684.

Swanson, R. W., Klepper, M. R., Lowell, W. R., Honkala, F. S., Cressman, E. R., Bostwick, D. A., Payne, O. A., and Ruppel, E. T., 1952 Stratigraphic sections of the Phosphoria formation in Montana, 1947-48: U. S. Geol. Survey Gireular 209. TEI-186 
DIAMOND DRILL HOLE 8, FORT HALL MINE, IDAHO. LOT NO. 1279.

Part of phosphatic shele member of Phosphoria formation cored in diamond drill hole 8 , near diamond drill hole 237 at Fort Hall Mine of J. R. Simplot Company, sec. 14, T. 4 S., R. 37 E., Bingham County, Idaho. Hole drilled in October 1948 by U. S. Bureau of Mines, chem. U by the U. S. Geological A. E. Long in charge, and core measured and sampled by D. F. Davidson. Samples analyzed by U. S. Bureau of Mines Laboratory, Albany, Survey Laboratory, Geochemistry
Oregon.

\begin{tabular}{|c|c|c|c|c|c|c|c|c|c|c|c|}
\hline \multirow{2}{*}{$\begin{array}{c}\text { Bed } \\
\text { no. }\end{array}$} & \multirow[t]{2}{*}{ Rock description } & \multirow{2}{*}{$\begin{array}{l}\text { Sample } \\
\text { no. }\end{array}$} & \multirow{2}{*}{$\begin{array}{c}\text { Thickness } \\
\text { (feet) }\end{array}$} & \multicolumn{2}{|c|}{$\begin{array}{l}\text { Chemical analyses } \\
\text { (percent) }\end{array}$} & \multirow{2}{*}{$\begin{array}{l}\text { Cumulative } \\
\text { thickness } \\
\text { (feet) }\end{array}$} & \multirow{2}{*}{$\begin{array}{l}\text { Thickness } \mathbf{x} \\
\text { percent } \mathrm{P}_{2} \mathrm{O}_{5} \\
\text { (cumulative) }\end{array}$} & \multicolumn{2}{|c|}{$\begin{array}{c}\text { Uranium content } \\
\text { (percent) }\end{array}$} & \multirow{2}{*}{$\begin{array}{l}\text { Thickness } \mathbf{x} \\
\text { percent eU } \\
\text { (cumulative) }\end{array}$} & $=$ \\
\hline & & & & $\mathrm{P}_{2} \mathrm{O}_{5}$ & $\begin{array}{c}\text { Acid } \\
\text { insoluble }\end{array}$ & & & eU & Chem. U & & \\
\hline \multicolumn{11}{|c|}{ Phosphatic shale member of Phosphoria formation-lower beds only } & \\
\hline $\begin{array}{ll}P & -35 \\
P & -34 \\
P & -33 \\
P & -32\end{array}$ & $\begin{array}{l}\text { Mudstone and phssphaie rock } \\
\text { Mudstone and phosphate rock } \\
\text { Limestone, argillaceous }\end{array}$ & $\begin{array}{l}\text { DFD-1144 } \\
\text { DFD-1145 } \\
\text { DFD-1146 }\end{array}$ & $\begin{array}{l}2.5 \\
0.9 \\
1.6\end{array}$ & $\begin{array}{r}16.1 \\
15.8 \\
5.2\end{array}$ & $\begin{array}{l}40.2 \\
41.5 \\
23.2\end{array}$ & $\begin{array}{l}2.5 \\
3.4 \\
5.0\end{array}$ & $\begin{array}{l}40.2 .5 \\
54.47 \\
62.79\end{array}$ & $\begin{array}{l}.003 \\
.004 \\
.002\end{array}$ & $\ddot{--}$ & $\begin{array}{l}.008 \\
.011 \\
.014\end{array}$ & \\
\hline $\begin{array}{ll}P & -32 \\
P & -31\end{array}$ & $\begin{array}{l}\text { Phosphate rock, argillaceous, } \\
\text { calcareous } \\
\text { Mudstone, phosphatic, calcareous }\end{array}$ & $\begin{array}{l}\text { DFD-1147 } \\
\text { DFD-1148 }\end{array}$ & $\begin{array}{l}0.7 \\
1.8\end{array}$ & $\begin{array}{r}15.4 \\
9.5\end{array}$ & $\begin{array}{l}33.8 \\
51.6\end{array}$ & $\begin{array}{l}5.7 \\
7.5\end{array}$ & $\begin{array}{l}73.57 \\
90.67\end{array}$ & $\begin{array}{l}.005 \\
.004\end{array}$ & .005 & $\cdot \begin{array}{l}.018 \\
.025\end{array}$ & 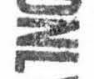 \\
\hline $\begin{array}{ll}P & -30 \\
P & -29 \\
P & -28 \\
P & -27 \\
P & -26\end{array}$ & $\begin{array}{l}\text { Limestane } \\
\text { Core missing } \\
\text { Mudstone, phosphatic } \\
\text { Mudstone, phosphatic } \\
\text { Phosphate fock, calcareous, argillaceous }\end{array}$ & $\begin{array}{l}\text { DFD-1149 } \\
-- \\
\text { DFD-1150 } \\
\text { DFD-1206 } \\
\text { DFD-1207 }\end{array}$ & $\begin{array}{l}0.8 \\
1.4 \\
0.3 \\
3.2 \\
1.5\end{array}$ & $\begin{array}{r}2.3 \\
-- \\
13.8 \\
13.3 \\
18.4\end{array}$ & $\begin{array}{l}10.2 \\
--. \\
40.5 \\
49.4 \\
24.4\end{array}$ & $\begin{array}{r}8.3 \\
9.7 \\
10.0 \\
13.2 \\
14.7\end{array}$ & $\begin{array}{l}\frac{92.51}{-. .14 *} \\
4.14 * \\
46.70 \\
74.30\end{array}$ & $\begin{array}{l}.0005 \\
.007 \\
.004 \\
.003\end{array}$ & $\begin{array}{l}-- \\
.006 \\
-- \\
--\end{array}$ & $\begin{array}{l}\frac{.025}{---} \\
.002 * \\
.015 \\
.019\end{array}$ & 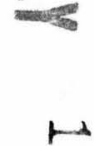 \\
\hline $\begin{array}{ll}P & -25 \\
P & -24 \\
P & -23 \\
P & -22 \\
P & -21\end{array}$ & $\begin{array}{l}\text { Phosphate rock, calcareous, argillaceous } \\
\text { Phosphiate rock, calcareous } \\
\text { Limestone, phosphatic } \\
\text { Phosphate rock } \\
\text { Phosphate rock, argillaceous }\end{array}$ & $\begin{array}{l}\text { DFD-1208 } \\
\text { DFD- } 1209 \\
\text { DFD-3336 } \\
\text { DFD-1248 } \\
\text { DFD-1249 }\end{array}$ & $\begin{array}{l}1.2 \\
1.0 \\
1.2 \\
1.8 \\
1.6\end{array}$ & $\begin{array}{l}14.8 \\
22.9 \\
15.6 \\
26.7 \\
16.2\end{array}$ & $\begin{array}{l}25.8 \\
19.6 \\
11.6 \\
19.6 \\
39.1\end{array}$ & $\begin{array}{l}15.9 \\
16.9 \\
18.1 \\
19.9 \\
21.5\end{array}$ & $\begin{array}{r}92.06 \\
114.96 \\
133.68 \\
181.74 \\
207.66\end{array}$ & $\begin{array}{l}.004 \\
.004 \\
.002 \\
.009 \\
.007\end{array}$ & $\begin{array}{l}-- \\
-- \\
-008 \\
.005\end{array}$ & $\begin{array}{l}.024 \\
.028 \\
.031 \\
.047 \\
.058\end{array}$ & \\
\hline $\begin{array}{ll}P & -20 \\
P & -19 \\
P & -18 \\
P & -17 \\
P & -16\end{array}$ & $\begin{array}{l}\text { Mudstone, phosphatic } \\
\text { Phosphate rock, argillaceous, calcareous } \\
\text { Phosphate rock, arglllaceous } \\
\text { Phosphate rock, argillaceous } \\
\text { Phosphate rock, argillaceous }\end{array}$ & $\begin{array}{l}\text { DFD-1250 } \\
\text { DFD-1251 } \\
\text { DFD-1252 } \\
\text { DFD-1253 } \\
\text { DFD }-1254\end{array}$ & $\begin{array}{l}1.2 \\
1.7 \\
0.6 \\
1.5 \\
1.8\end{array}$ & $\begin{array}{l}15.6 \\
17.9 \\
25.8 \\
27.0 \\
26.3\end{array}$ & $\begin{array}{l}43.4 \\
32.3 \\
21.9 \\
21.0 \\
22.8\end{array}$ & $\begin{array}{l}22.7 \\
24.4 \\
25.0 \\
26.5 \\
28.3\end{array}$ & $\begin{array}{l}226.38 \\
256.81 \\
272.29 \\
312.79 \\
360.13 \\
\end{array}$ & $\begin{array}{l}.007 \\
.007 \\
.005 \\
.008 \\
.010\end{array}$ & $\begin{array}{l}.006 \\
.006 \\
.005 \\
.007 \\
.009\end{array}$ & $\begin{array}{l}.066 \\
.078 \\
.081 \\
.093 \\
.111 \\
\end{array}$ & \\
\hline $\begin{array}{ll}P & -15 \\
P & -14 \\
P & -13 \\
P & -12 \\
P & -11\end{array}$ & $\begin{array}{l}\text { Core missing } \\
\text { Mudstone, phosphatic } \\
\text { Mudstune, phosphatic } \\
\text { Phosphate rock } \\
\text { Phc.sphate rock, argillaceous }\end{array}$ & $\begin{array}{l}\text { DFD- } 1255 \\
\text { DFD-1256 } \\
\text { DFD-1257 } \\
\text { DFD-1258 }\end{array}$ & $\begin{array}{l}2.8 \\
0.5 \\
0.6 \\
1.3 \\
2.8\end{array}$ & $\begin{array}{l}10.6 \\
15.7 \\
30.7 \\
22.2\end{array}$ & $\begin{array}{l}45.6 \\
46.5 \\
13.8 \\
33.1\end{array}$ & $\begin{array}{l}31.1 \\
31.6 \\
32.2 \\
33.5 \\
36.3\end{array}$ & $\begin{array}{r}-- \\
8.30 * \\
17.72 \\
57.63 \\
119.79\end{array}$ & $\begin{array}{l}.006 \\
.007 \\
.008 \\
.007\end{array}$ & $\begin{array}{l}.0 \\
.005 \\
.005 \\
.009 \\
.005\end{array}$ & $\begin{array}{l}-. \\
.003 * \\
.007 \\
.018 \\
.037\end{array}$ & \\
\hline $\begin{array}{l}P=10 \\
P=9 \\
P=8 \\
P=7\end{array}$ & $\begin{array}{l}\text { Phosphate rock and mudstone } \\
\text { Phosphate rock and mudstone } \\
\text { Phosphate rock and mudstone } \\
\text { Limestone }\end{array}$ & $\begin{array}{l}\text { DFD-1259 } \\
\text { DFD-1827 } \\
\text { DFD-1828 } \\
\text { DFD-1976 }\end{array}$ & $\begin{array}{l}2.0 \\
2.0 \\
1.0 \\
1.0\end{array}$ & $\begin{array}{r}18.5 \\
30.8 \\
24.6 \\
4.9\end{array}$ & $\begin{array}{l}28.1 \\
12.6 \\
20.2 \\
17.4\end{array}$ & $\begin{array}{l}38.3 \\
40.3 \\
41.3 \\
42.3\end{array}$ & $\begin{array}{l}156.79 \\
218.39 \\
242.99 \\
247.89\end{array}$ & $\begin{array}{l}.008 \\
.011 \\
.008 \\
.003\end{array}$ & $\begin{array}{l}.007 \\
.009 \\
.006\end{array}$ & $\begin{array}{l}.053 \\
.075 \\
.083 \\
.086\end{array}$ & \\
\hline
\end{tabular}




\begin{tabular}{|c|c|c|c|c|c|c|c|c|c|c|}
\hline \multirow{2}{*}{$\begin{array}{c}\text { Bed } \\
\text { no. }\end{array}$} & \multirow{2}{*}{ Rock doscription } & \multirow{2}{*}{$\begin{array}{c}\text { Sample } \\
\text { no. }\end{array}$} & \multirow{2}{*}{$\begin{array}{l}\text { Thickness } \\
\text { (feet) }\end{array}$} & \multicolumn{2}{|c|}{$\begin{array}{c}\text { Chemical analyses } \\
\text { (percent) }\end{array}$} & \multirow{2}{*}{$\begin{array}{l}\text { Cumulative } \\
\text { thickness } \\
\text { (feet) }\end{array}$} & \multirow{2}{*}{$\begin{array}{l}\text { Thickness } x \\
\text { percent } \mathbf{P}_{2} \mathbf{O}_{5} \\
\text { (cumulative) }\end{array}$} & \multicolumn{2}{|c|}{$\begin{array}{l}\text { Uranium content } \\
\text { (porcent) }\end{array}$} & \multirow{2}{*}{$\begin{array}{l}\text { Thickness } \mathbf{x} \\
\text { percent eU } \\
\text { (cumulative) }\end{array}$} \\
\hline & & & & $\mathrm{P}_{2} \mathrm{O}_{5}$ & $\begin{array}{c}\text { Acd } \\
\text { insoluble }\end{array}$ & & & eu & Chem. V & \\
\hline $\begin{array}{l}P=6 \\
P=5 \\
P=-4 \\
P=3 \\
P=2\end{array}$ & $\begin{array}{l}\text { Phosphate rock } \\
\text { Limestone, phosphatic } \\
\text { Phosphate rock, argillaceous } \\
\text { Phosphate rock } \\
\text { Limestone, argillaceous, phosphatic }\end{array}$ & $\begin{array}{l}\text { DFD-1977 } \\
\text { DFD-1978 } \\
\text { DFD-1979 } \\
\text { DFD-1980 } \\
\text { DFD-2170 }\end{array}$ & $\begin{array}{l}1.1 \\
1.8 \\
2.0 \\
2.6 \\
0.5\end{array}$ & $\begin{array}{l}28.0 \\
11.8 \\
24.0 \\
34.7 \\
11.8\end{array}$ & $\begin{array}{r}15.9 \\
13.1 \\
23.9 \\
3.1 \\
29.4\end{array}$ & $\begin{array}{l}43.4 \\
45.2 \\
47.2 \\
49.8 \\
50.3\end{array}$ & $\begin{array}{l}278.69 \\
299.93 \\
347.93 \\
438.15 \\
444.05\end{array}$ & $\begin{array}{l}.007 \\
.004 \\
.007 \\
.008 \\
.005\end{array}$ & $\begin{array}{l}.008 \\
.005 \\
.000 \\
.004\end{array}$ & $\begin{array}{l}.094 \\
.101 \\
.115 \\
.136 \\
.138\end{array}$ \\
\hline $\mathbf{P}-\mathbf{1}$ & Limestone, argillaceous & DFD-2295 & 0.8 & 5.4 & 33.3 & 51.1 & $448.37 * *$ & .002 & $-\cdot$ & $.140 * *$ \\
\hline \multicolumn{11}{|c|}{ Wells formation } \\
\hline $\mathrm{Cw}-1$ & Limestone, arglllaceous & DFD-2296 & 0.2 & 2.5 & 23.0 & 0.2 & 0.50 & $\cdots$ & -- & -. \\
\hline
\end{tabular}


DIAMOND DRILL hOLE 9, FORT HALL MINE, IDAHO. LOT NO. 1280.

Part of phosphatic shale momber of Phosphoria formation sampled from diamond drill hole 9 near diamond drill hole 237 at For Mines, A. F. Lone in charge, and measured and eampled by D. F. Davidson. Samples analyzed by U. S. Bureau of Mines Laboratory, Albany, Orogon.

\begin{tabular}{|c|c|c|c|c|c|c|c|c|c|}
\hline \multirow{2}{*}{$\begin{array}{l}\text { Bed } \\
\text { no. }\end{array}$} & \multirow{2}{*}{ Rock description } & \multirow{2}{*}{$\begin{array}{l}\text { Sample } \\
\text { no. }\end{array}$} & \multirow{2}{*}{$\begin{array}{c}\text { Thicknesss } \\
\text { (foet) }\end{array}$} & \multicolumn{2}{|c|}{$\begin{array}{c}\text { Chemical analyses } \\
\text { (percent) }\end{array}$} & \multirow{2}{*}{$\begin{array}{l}\text { Cumulative } \\
\text { thickness } \\
\text { (feot) }\end{array}$} & \multirow{2}{*}{$\begin{array}{l}\text { Thickness } x \\
\text { percent } \mathbf{P}_{2} \mathrm{O}_{5} \\
\text { (cumulativo) }\end{array}$} & $\begin{array}{l}\text { Uranium content } \\
\text { (percent) }\end{array}$ & \multirow{2}{*}{$\begin{array}{l}\text { Thickness } \mathbf{x} \\
\text { percent eU } \\
\text { (cumulative) }\end{array}$} \\
\hline & & & & $\mathrm{P}_{2} \mathrm{O}_{5}$ & $\begin{array}{c}\text { Acid } \\
\text { insoluble }\end{array}$ & & & of |Chem. U & \\
\hline
\end{tabular}

Phosphatic shale member of Phosphoria formation-lower beds only

\begin{tabular}{|c|c|}
\hline $\begin{array}{ll}P & -34 \\
P & -33 \\
P & -32 \\
P & -31 \\
P & -30\end{array}$ & $\begin{array}{l}\text { Mudatone, calcareous } \\
\text { Mudstone, phosphatic } \\
\text { Mudatone; phosphatic, calcareous } \\
\text { Limestone } \\
\text { Mudetone, phosphatic, calcareous }\end{array}$ \\
\hline $\begin{array}{l}-29 \\
-28 \\
-27 \\
-26 \\
-25\end{array}$ & $\begin{array}{l}\text { Core misaing } \\
\text { Limestone } \\
\text { Mudstone, phosphatic } \\
\text { Phosphate rock, argillaceous } \\
\text { Phosphate rock, calcareous, argilleceous }\end{array}$ \\
\hline $\begin{array}{l}-24 \\
-23 \\
-22 \\
-21 \\
-20\end{array}$ & 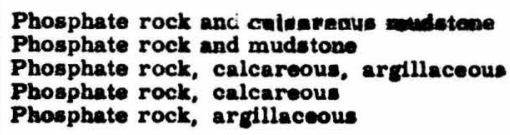 \\
\hline $\begin{array}{l}-19 \\
-18 \\
-17 \\
-16 \\
-15\end{array}$ & $\begin{array}{l}\text { Phosphate rock, argillaceous } \\
\text { Limiastee } \\
\text { Mudetone, phosphatic, calcareous } \\
\text { Phos phate rock and mudstone } \\
\text { Phosphate rock and mudatone }\end{array}$ \\
\hline $\begin{array}{l}-14 \\
-13 \\
-12 \\
-11 \\
-10\end{array}$ & $\begin{array}{l}\text { Phosphate rock and mudstone } \\
\text { Limestone } \\
\text { Core missing } \\
\text { Mudstone, phosphatic, calcareous } \\
\text { Phosphate rock and mudatone }\end{array}$ \\
\hline $\begin{array}{l}: 9 \\
: 8 \\
: 7 \\
:-6 \\
-5\end{array}$ & $\begin{array}{l}\text { Phosphate rock and mudstone } \\
\text { Phosphate rock } \\
\text { Phosphate rock and mudetono } \\
\text { Limestone, argillaceous } \\
\text { Phosphate rock, calcareous }\end{array}$ \\
\hline
\end{tabular}

\begin{tabular}{|l|} 
DFD-2497 \\
DFD-2298 \\
DFD-2299 \\
DFD-2300 \\
DFD-3035 \\
-- \\
DFD-2468 \\
DFD-2469 \\
DFD-2470 \\
DFD-2471 \\
DFD-2472 \\
DFD-2173 \\
DFD-2474 \\
DFD-2475 \\
DFD-2531 \\
DFD-2532 \\
DFD-2533 \\
DFD-2534 \\
DFD-2535 \\
DFD-2536 \\
DFD-2537 \\
DFD-2538 \\
DFD-2539 \\
DFD-2540 \\
DFD-2541 \\
DFD-2542 \\
DFD-2543 \\
DFD-2544 \\
DFD-2545
\end{tabular}

\begin{tabular}{r|r|r|}
0.5 & 7.7 & 43.8 \\
2.0 & 13.5 & 44.0 \\
0.6 & 13.7 & 36.5 \\
0.7 & 1.9 & 8.0 \\
3.4 & 11.5 & 46.2 \\
1.0 &.- &.- \\
0.8 & 0.7 & 8.6 \\
2.2 & 12.1 & 49.4 \\
1.1 & 16.0 & 39.7 \\
1.0 & 14.6 & 30.6 \\
0.9 & 19.3 & 21.7 \\
1.0 & 18.2 & 22.9 \\
1.5 & 17.0 & 23.5 \\
1.2 & 24.4 & 16.9 \\
1.7 & 26.1 & 20.4 \\
1.6 & 17.2 & 38.6 \\
0.9 & 3.2 & 4.5 \\
2.9 & 13.4 & 36.5 \\
1.6 & 29.1 & 13.1 \\
1.9 & 22.7 & 29.6 \\
1.3 & 22.6 & 27.3 \\
0.5 & 2.6 & 14.5 \\
0.4 & $1 .$. &.- .7 \\
2.5 & 13.9 & 40.7 \\
2.7 & 27.6 & 19.8 \\
2.0 & 22.8 & 31.6 \\
2.0 & 31.7 & 9.4 \\
2.2 & 27.6 & 16.3 \\
0.6 & 0.9 & 22.4 \\
1.6 & 22.0 & 19.0 \\
\hline
\end{tabular}

\begin{tabular}{|l|}
\hline 0.5 \\
2.5 \\
3.1 \\
3.8 \\
7.2 \\
8.2 \\
9.0 \\
11.2 \\
12.3 \\
13.3 \\
14.2 \\
15.2 \\
16.7 \\
17.9 \\
19.6 \\
21.2 \\
22.1 \\
25.0 \\
26.6 \\
28.5 \\
29.8 \\
30.3 \\
30.7 \\
33.2 \\
35.9 \\
37.9 \\
39.9 \\
42.1 \\
42.7 \\
44.3
\end{tabular}

\begin{tabular}{r}
3.85 \\
30.85 \\
39.07 \\
40.40 \\
79.50 \\
\hline $0.76 *$ \\
$0.56 *$ \\
27.18 \\
4.78 \\
59.38 \\
76.75 \\
94.95 \\
120.45 \\
149.73 \\
194.10 \\
221.62 \\
224.50 \\
263.36 \\
309.92 \\
353.05 \\
382.43 \\
383.73 \\
\hline $34.75 *$ \\
109.27 \\
154.87 \\
218.27 \\
288.99 \\
279.53 \\
314.73
\end{tabular}

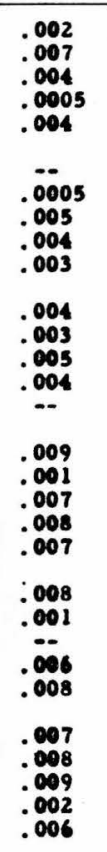

\begin{tabular}{|c|c|}
\hline \begin{tabular}{c}
$\ddot{003}$ \\
\hdashline- \\
--
\end{tabular} & $\begin{array}{l}.001 \\
.015 \\
.017 \\
.018 \\
.031\end{array}$ \\
\hline $\begin{array}{c}\because \\
.004 \\
\because \therefore \\
\therefore\end{array}$ & $\begin{array}{l}.0 \\
.000 * \\
.011 \\
.016 \\
.019\end{array}$ \\
\hline $\begin{array}{c}\because \\
\ddot{0003} \\
\because-\end{array}$ & $\begin{array}{l}.022 \\
.025 \\
.033 \\
.038 \\
--\end{array}$ \\
\hline $\begin{array}{l}.009 \\
.005 \\
.006 \\
.006 \\
.006\end{array}$ & $\begin{array}{l}.014 * \\
.015 \\
.036 \\
.048 \\
.062\end{array}$ \\
\hline & $\begin{array}{r}.072 \\
.073 \\
\end{array}$ \\
\hline $\begin{array}{l}.005 \\
.006\end{array}$ & $\begin{array}{l}.015 * \\
.037\end{array}$ \\
\hline $\begin{array}{l}.005 \\
.006 \\
.007 \\
.005\end{array}$ & $\begin{array}{l}.051 \\
.067 \\
.086 \\
.088 \\
.097\end{array}$ \\
\hline
\end{tabular}

Samples analyzed for $\mathrm{eU}$ and che S. Geological

- Cumulative deta incomplote due to misaing information. Computations start from zero after intermuption. 


\begin{tabular}{|c|c|c|c|c|c|c|c|c|c|c|}
\hline \multirow{2}{*}{$\begin{array}{c}\text { Bed } \\
\text { no. }\end{array}$} & \multirow[t]{2}{*}{ Rock description } & \multirow{2}{*}{$\begin{array}{c}\text { Sample } \\
\text { no. }\end{array}$} & \multirow{2}{*}{$\begin{array}{c}\text { Thickness } \\
\text { (feet) }\end{array}$} & \multicolumn{2}{|c|}{$\begin{array}{c}\text { Chemical analyses } \\
\text { (percent) }\end{array}$} & \multirow{2}{*}{$\begin{array}{l}\text { Cumulative } \\
\text { thickness } \\
\text { (feet) }\end{array}$} & \multirow{2}{*}{$\begin{array}{l}\text { Thickness } x \\
\text { percent } \mathbf{P}_{2} \mathrm{O}_{5} \\
\text { (cumulative) }\end{array}$} & \multicolumn{2}{|c|}{$\begin{array}{l}\text { Uranium content } \\
\text { (percent) }\end{array}$} & \multirow{2}{*}{$\begin{array}{l}\text { Thickness X } \\
\text { percent eU } \\
\text { (cumulative) }\end{array}$} \\
\hline & & & & $\mathrm{P}_{2} \mathrm{O}_{5}$ & $\begin{array}{c}\text { Acid } \\
\text { insoluble }\end{array}$ & & & बर्u & Chom. U & \\
\hline $\begin{array}{l}P=4 \\
P=3 \\
P=2 \\
P=1\end{array}$ & $\begin{array}{l}\text { Limestone } \\
\text { Core missing } \\
\text { Phosphate rock } \\
\text { Limestone, argillaceous }\end{array}$ & $\begin{array}{l}\text { DFD-2546 } \\
-- \\
\text { DFD }-2547 \\
\text { DFD }-2548\end{array}$ & $\begin{array}{l}0.8 \\
2.9 \\
1.4 \\
1.4\end{array}$ & $\begin{array}{r}6.4 \\
27.5 \\
0.8\end{array}$ & $\begin{array}{r}8.6 \\
11.2 \\
36.0\end{array}$ & $\begin{array}{l}45.1 \\
48.1 \\
49.4 \\
50.8\end{array}$ & $\begin{array}{l}\frac{319.85}{--} \\
35.75 * \\
36.87 * *\end{array}$ & $\begin{array}{l}.003 \\
.005 \\
.001\end{array}$ & $\begin{array}{l}\ddot{-} \\
.004\end{array}$ & $\begin{array}{l}\frac{.100}{--} \\
.006 * \\
.008 * *\end{array}$ \\
\hline \multicolumn{11}{|c|}{ Wells formation } \\
\hline $\begin{array}{l}\text { Cw- 3 } \\
\text { Cw-2 } \\
\text { Cw- 1 }\end{array}$ & $\begin{array}{l}\text { Limestone, arglllaceous } \\
\text { Core missing } \\
\text { Limestone, argillaceous }\end{array}$ & $\begin{array}{l}\text { DFD }-2549 \\
-- \\
\text { DFD }-2550\end{array}$ & $\begin{array}{l}0.7 \\
3.0 \\
1.4\end{array}$ & $\begin{array}{l}2.7 \\
-\because \\
2.1\end{array}$ & $\begin{array}{l}21.2 \\
31.7\end{array}$ & $\begin{array}{l}0.7 \\
3.7 \\
5.1\end{array}$ & $\frac{1.89}{4.83 *}$ & $\begin{array}{l}.002 \\
.0005\end{array}$ & $\begin{array}{l}\because- \\
\because-\end{array}$ & $\frac{.001}{.002 *}$ \\
\hline
\end{tabular}

* Cumulative data incomplete due to missing information. Computations start from zero after interruption. 
CALDWELl CANYON, IDAHO. LOT NO. 1260.

Phosphatic shale member of Phosphorla formation sampled in bulldozer trench on north side of Caldwell Canyon, sec. 1, T. $8 \mathrm{~S}$., R. 43 E., Caribou County, Idaho, on east limb of Slug Creek syncline. Beds strike N. $10^{\circ} \mathrm{W}$. and dip $40^{\circ} \mathrm{E}$. Section measured by R. A. Hoppin, D. F. Davidson, insoluble by U. S. Bureau of Mines Laboratory, Albany, Oregon, and for other constituents by Trace Elements Section Laboratory, U. S. Geological Survey, Washington, D. C.

\begin{tabular}{|c|c|c|c|c|c|c|c|c|c|c|c|c|c|}
\hline \multirow{2}{*}{$\begin{array}{l}\text { Bed } \\
\text { no. }\end{array}$} & \multirow{2}{*}{ Rock description } & \multirow{2}{*}{$\begin{array}{l}\text { Sample } \\
\text { no. }\end{array}$} & \multirow{2}{*}{$\begin{array}{c}\text { Thickness } \\
\text { (feet) }\end{array}$} & \multicolumn{5}{|c|}{ Chemical analyses (percent) } & \multirow{2}{*}{$\begin{array}{l}\text { Cumulative } \\
\text { thlckness } \\
\text { (foet) }\end{array}$} & \multirow{2}{*}{$\begin{array}{l}\text { Thickness } x \\
\text { percent } P_{2} O_{5} \\
\text { (cumulative) }\end{array}$} & \multirow{2}{*}{\multicolumn{2}{|c|}{$\begin{array}{c}\text { Uranium content } \\
\text { (percent) }\end{array}$}} & \multirow{2}{*}{$\begin{array}{l}\text { Thickness } x \\
\text { percent eU } \\
\text { (cumulative) }\end{array}$} \\
\hline & & & & $\mathbf{P}_{\mathbf{2}} \mathrm{O}_{\mathbf{5}}$ & $\mathrm{Al}_{2} \mathrm{O}_{3}$ & $\mathrm{Fe}_{2} \mathrm{O}_{3}$ & $\begin{array}{l}\text { Loss on } \\
\text { Ignition }\end{array}$ & $\begin{array}{c}\text { Acid } \\
\text { insoluble }\end{array}$ & & & & & \\
\hline \multicolumn{14}{|c|}{ Rex member of Phosphoria formation-basal bed only } \\
\hline \multicolumn{14}{|c|}{ Phosphatic shale member of Phosphoria formation ${ }^{1}$} \\
\hline $\begin{array}{l}\text { P-84 } \\
\text { P-83 } \\
\text { P-82 } \\
\text { P-81 } \\
\text { P-80 }\end{array}$ & $\begin{array}{l}\text { Mudatone } \\
\text { Mudstone } \\
\text { Mudatone } \\
\text { Mudotone, foo. col. no. } 4 \text {-JES-1892 } \\
\text { Mudatoon, calcaroous }\end{array}$ & $\begin{array}{l}\text { WOM-1749 } \\
\text { WOM-1748 } \\
\text { WOM-1747 } \\
\text { WOM-1746 } \\
\text { WOM-1745 }\end{array}$ & $\begin{array}{l}1.1 \\
1.2 \\
1.4 \\
2.7 \\
0.6\end{array}$ & $\begin{array}{l}5.2 \\
0.7 \\
4.9 \\
0.5 \\
2.4\end{array}$ & $\begin{array}{l}-- \\
-- \\
-- \\
--\end{array}$ & $\begin{array}{l}-- \\
-- \\
--\end{array}$ & $\begin{array}{l}-- \\
-- \\
--\end{array}$ & $\begin{array}{l}57.7 \\
70.0 \\
59.0 \\
64.4 \\
63.1\end{array}$ & $\begin{array}{l}1.1 \\
2.3 \\
3.7 \\
6.4 \\
7.0\end{array}$ & $\begin{array}{r}5.70 \\
6.56 \\
13.42 \\
14.77 \\
16.21\end{array}$ & $\begin{array}{l}.004 \\
.002 \\
.005 \\
.002 \\
.003\end{array}$ & $\begin{array}{l}-- \\
\ddot{0} \\
005 \\
--\end{array}$ & $\begin{array}{l}.004 \\
.007 \\
.014 \\
.019 \\
.021\end{array}$ \\
\hline$P-79$ & \multirow{2}{*}{$\begin{array}{l}\text { Limestone, argillacoous; fos. col. } \\
\text { no. 18-JEs-188 } \\
\text { Mudetone, calcareous, fos. col. no. } \\
\text { 48-JES-187 } \\
\text { Mudetone, phoephatic } \\
\text { Mudetone } \\
\text { Phosphate rock, argillaoeous }\end{array}$} & WOM-1744 & 1.1 & 0.6 & -- & -- & -- & 38.0 & 8.1 & 16.87 & .002 & -- & .023 \\
\hline $\begin{array}{l}P-78 \\
P-77 \\
P-76 \\
P-75\end{array}$ & & $\begin{array}{l}\text { WOM-1743 } \\
\text { WOM-1742 } \\
\text { WOM-1741 } \\
\text { WOM-1740 }\end{array}$ & $\begin{array}{l}3.3 \\
0.3 \\
1.3 \\
0.5\end{array}$ & $\begin{array}{r}2.7 \\
14.8 \\
2.3 \\
24.1\end{array}$ & $\begin{array}{l}\because- \\
\because- \\
-\end{array}$ & $\begin{array}{l}\because \\
- \\
-\end{array}$ & $\begin{array}{l}- \\
- \\
--\end{array}$ & $\begin{array}{l}68.3 \\
49.0 \\
78.5 \\
21.2\end{array}$ & $\begin{array}{l}11.4 \\
11.7 \\
13.0 \\
13.5\end{array}$ & $\begin{array}{l}25.78 \\
30.22 \\
33.21 \\
45.26\end{array}$ & $\begin{array}{l}.003 \\
.005 \\
.003 \\
.009\end{array}$ & $\begin{array}{l}.003 \\
.004 \\
.008\end{array}$ & $\begin{array}{l}.033 \\
.035 \\
.038 \\
.043\end{array}$ \\
\hline $\begin{array}{l}P-74 \\
P-73\end{array}$ & \multirow{2}{*}{$\begin{array}{l}\text { Mudatono; fos. col. no. 48-JES-186 } \\
\text { Mudatone; ealcareous; fos. col. no. } \\
\text { 48-JES-185 } \\
\text { Phouphate rook } \\
\text { Phouphate rock; fos. col. no. 48-JEs-184 } \\
\text { Mudotone }\end{array}$} & WOM-1780 & 1.9 & 2.5 & -- & $\cdots$ & $\cdots$ & 69.5 & 15.4 & 50.01 & .004 & -- & .051 \\
\hline $\begin{array}{l}P-72 \\
P-71 \\
P-70\end{array}$ & & $\begin{array}{l}\text { WOM-1779 } \\
\text { WOM } 1778 \\
\text { WOM-1777 } \\
\text { WOM-1776 }\end{array}$ & $\begin{array}{l}1.3 \\
1.9 \\
0.7 \\
0.6\end{array}$ & $\begin{array}{r}0.2 \\
32.9 \\
29.1 \\
7.5\end{array}$ & $\begin{array}{l}-- \\
-- \\
--\end{array}$ & $\begin{array}{l}-- \\
-- \\
--\end{array}$ & $\begin{array}{l}-- \\
-- \\
--\end{array}$ & $\begin{array}{l}52.0 \\
11.3 \\
17.7 \\
58.2\end{array}$ & $\begin{array}{l}16.7 \\
18.6 \\
19.3 \\
19.9\end{array}$ & $\begin{array}{r}50.27 \\
112.78 \\
133.15 \\
137.65\end{array}$ & $\begin{array}{l}.001 \\
.012 \\
.010 \\
.005\end{array}$ & $\begin{array}{r}\ddot{-0} \\
.010 \\
.008 \\
.004\end{array}$ & $\begin{array}{l}.052 \\
.075 \\
.082 \\
.085\end{array}$ \\
\hline $\begin{array}{l}\text { P-69 } \\
\text { P-68 } \\
--\end{array}$ & \multirow{3}{*}{$\begin{array}{l}\text { Phoophate rock, calcareous } \\
\text { Phoophate rock } \\
\text { Limestone concretion; fos. col. no. } \\
\text { 48-JEs-183 } \\
\text { Phosphate rock, celcareous and phosphatic } \\
\text { calcareous mudatones fos. col. no. } \\
\text { 48-JES-182 } \\
\text { Mudatone, phosphatic }\end{array}$} & $\begin{array}{l}\text { WOM-1775 } \\
\text { WOM-1774 }\end{array}$ & $\begin{array}{l}0.9 \\
0.8\end{array}$ & $\begin{array}{l}24.9 \\
33.3\end{array}$ & $\ddot{-}$ & $\ddot{-}$ & $\because$ & $\begin{array}{l}9.5 \\
5.5\end{array}$ & $\begin{array}{l}20.8 \\
21.6\end{array}$ & $\begin{array}{l}160.06 \\
186.70\end{array}$ & $\begin{array}{l}.008 \\
.012\end{array}$ & .006 & .092 \\
\hline$P-67$ & & WOM-1773 & $(0.9)$ & 5.3 & -- & -- & $\cdots$ & 2.2 & -- & -- & .002 & -- & -- \\
\hline P-66 & & $\begin{array}{l}\text { WOM-1772 } \\
\text { WOM-1771 }\end{array}$ & $\begin{array}{l}3.0 \\
1.0\end{array}$ & $\begin{array}{l}19.7 \\
12.7\end{array}$ & $\begin{array}{l}-- \\
--\end{array}$ & $\begin{array}{l}-- \\
--\end{array}$ & -- & $\begin{array}{l}25.0 \\
45.0\end{array}$ & $\begin{array}{l}24.6 \\
25.6\end{array}$ & $\begin{array}{l}245.80 \\
258.50\end{array}$ & $\begin{array}{l}.008 \\
.007\end{array}$ & .006 & .126 \\
\hline \multirow[t]{2}{*}{$\begin{array}{l}P-65 \\
P-64\end{array}$} & $\begin{array}{l}\text { Phosphate rock, arrillaceous } \\
\text { Mudatone; los. col. no. 48-JES-181 }\end{array}$ & $\begin{array}{l}\text { DFD- } 1790 \\
\text { DFD- } 1789\end{array}$ & 1.3 & $\begin{array}{r}18.0 \\
7.6\end{array}$ & $\because$ & 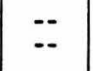 & $=$ & $\begin{array}{l}37.3 \\
63.3\end{array}$ & $\begin{array}{l}26.9 \\
28.2\end{array}$ & $\begin{array}{l}281.90 \\
291.78\end{array}$ & $\begin{array}{l}.007 \\
.005\end{array}$ & $\begin{array}{l}.005 \\
.004\end{array}$ & .142 \\
\hline & $\begin{array}{l}1 \text { The thinness of the Phosphatic shale m } \\
2 \text { Foasil collection by J. E. Smedley, Pa }\end{array}$ & ser is prob & due to & lting. & U. s. c & 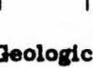 & 10 & & & & & & \\
\hline
\end{tabular}




\begin{tabular}{|c|c|c|c|c|c|c|c|c|c|c|c|c|c|}
\hline \multirow{2}{*}{$\begin{array}{c}\text { Bed } \\
\text { no. }\end{array}$} & \multirow{2}{*}{ Rock description } & \multirow{2}{*}{$\begin{array}{l}\text { Sample } \\
\text { no. }\end{array}$} & \multirow{2}{*}{$\begin{array}{c}\text { Thickness } \\
\text { (feet) }\end{array}$} & \multicolumn{5}{|c|}{ Chemical analyses (percent) } & \multirow{2}{*}{$\begin{array}{l}\text { Cumulative } \\
\text { thickness } \\
\text { (feet) }\end{array}$} & \multirow{2}{*}{$\begin{array}{l}\text { Thickness } x \\
\text { porcent } \mathbf{P}_{2} \mathrm{O}_{5} \\
\text { (cumulative) }\end{array}$} & \multicolumn{2}{|c|}{$\begin{array}{c}\text { Uranium content } \\
\text { (percent) }\end{array}$} & \multirow{2}{*}{$\begin{array}{l}\text { Thickness } x \\
\text { percent eU } \\
\text { (cumulative) }\end{array}$} \\
\hline & & & & $\mathrm{P}_{2} \mathrm{O}_{5}$ & $\mathrm{Al}_{2} \mathrm{O}_{3}$ & $\mathrm{~F} \bullet_{2} \mathrm{O}_{3}$ & $\begin{array}{l}\text { Loss on } \\
\text { ignition }\end{array}$ & $\begin{array}{l}\text { Acid } \\
\text { insoluble }\end{array}$ & & & ever & Chem. U & \\
\hline$P-63$ & 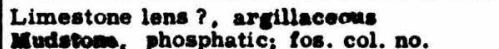 & DFD- 1788 & $(0.9)$ & 0.5 & -- & -- & -- & 29.8 & -- & -- & .0005 &.- & -- \\
\hline $\begin{array}{l}P-62 \\
P-61\end{array}$ & $\begin{array}{l}\text { 4t-JES-180 } \\
\text { Mudstone, phosphatic } \\
\text { Phosphate rock, argillaceous, fos. col. }\end{array}$ & $\begin{array}{l}\text { DFD- } 1787 \\
\text { DFD- } 1786\end{array}$ & $\begin{array}{l}3.2 \\
0.7\end{array}$ & $\begin{array}{r}8.5 \\
10.6\end{array}$ & $\because-$ & $\begin{array}{l}-- \\
--\end{array}$ & $\begin{array}{l}-- \\
--\end{array}$ & $\begin{array}{l}61.7 \\
59.8\end{array}$ & $\begin{array}{l}31.40 \\
32.10\end{array}$ & $\begin{array}{l}318.98 \\
326.40\end{array}$ & $\begin{array}{l}.005 \\
.005\end{array}$ & $\begin{array}{l}.003 \\
.003\end{array}$ & $\begin{array}{l}.164 \\
.168\end{array}$ \\
\hline P-60 & $\begin{array}{l}\text { no. 48-JES-179 } \\
\text { Mudstone, phosphatic }\end{array}$ & $\begin{array}{l}\text { DFD- } 1785 \\
\text { DFD- } 1784\end{array}$ & $\begin{array}{l}2.7 \\
0.5\end{array}$ & $\begin{array}{r}19.4 \\
8.9\end{array}$ & $\because-$ & $\begin{array}{l}-- \\
--\end{array}$ & -: & $\begin{array}{l}44.0 \\
67.3\end{array}$ & $\begin{array}{l}34.80 \\
35.30\end{array}$ & $\begin{array}{l}378.78 \\
383.23\end{array}$ & $\begin{array}{l}.004 \\
.004\end{array}$ & $\begin{array}{l}-- \\
--\end{array}$ & $\begin{array}{l}.178 \\
.180\end{array}$ \\
\hline $\begin{array}{l}P-58 \\
P-57 \\
P-56 \\
P-55\end{array}$ & $\begin{array}{l}\text { Mudstone, phosphatic, fos. col. no. } \\
\text { 48-JES-178 } \\
\text { Mudstone, phosphatic } \\
\text { Mudstone, Pos. col. no. 48-JES-177 } \\
\text { Mudstone } \\
\text { Mudstone }\end{array}$ & $\begin{array}{l}\text { DFD- } 1783 \\
\text { DFD- } 1782 \\
\text { DFD- } 1781 \\
\text { DFD- } 1760 \\
\text { DFD- } 1759\end{array}$ & $\begin{array}{l}0.9 \\
0.4 \\
3.6 \\
0.8 \\
0.5\end{array}$ & $\begin{array}{l}8.7 \\
9.6 \\
6.4 \\
1.6 \\
6.4\end{array}$ & $\begin{array}{l}-- \\
\because- \\
\because-\end{array}$ & $\begin{array}{l}-- \\
- \\
\because- \\
-\end{array}$ & $\begin{array}{l}-- \\
- \\
- \\
-\end{array}$ & $\begin{array}{l}66.3 \\
59.7 \\
70.6 \\
83.0 \\
74.2\end{array}$ & $\begin{array}{l}36.20 \\
36.60 \\
40.20 \\
41.00 \\
41.50\end{array}$ & $\begin{array}{l}391.06 \\
394.90 \\
417.94 \\
419.22 \\
422.42\end{array}$ & $\begin{array}{l}.005 \\
.004 \\
.003 \\
.002 \\
.003\end{array}$ & $\begin{array}{l}.003 \\
-- \\
-- \\
--\end{array}$ & $\begin{array}{l}.185 \\
.187 \\
.197 \\
.199 \\
.200\end{array}$ \\
\hline $\begin{array}{l}\text { P-54 } \\
\text { P-53 } \\
\text { P-52 } \\
\text { P-51 } \\
\text { P-50 }\end{array}$ & $\begin{array}{l}\text { Mudstone, phosphatic } \\
\text { Phosphate rock, arglliaceous } \\
\text { Mudstone, phosphatic } \\
\text { Mudstone, phosphatic } \\
\text { Mudstone }\end{array}$ & $\begin{array}{l}\text { DFD- } 1758 \\
\text { DFD- } 1757 \\
\text { DFD- } 1756 \\
\text { DFD- } 1755 \\
\text { DFD- } 1754\end{array}$ & $\begin{array}{l}1.2 \\
0.6 \\
0.8 \\
0.6 \\
0.7\end{array}$ & $\begin{array}{r}9.1 \\
17.5 \\
15.4 \\
9.8 \\
6.4\end{array}$ & $\begin{array}{l}-- \\
- \\
-- \\
-\end{array}$ & $\begin{array}{l}-- \\
- \\
\because- \\
-\end{array}$ & $\begin{array}{l}\because- \\
\because \\
\because \\
-\end{array}$ & $\begin{array}{l}64.0 \\
43.2 \\
45.8 \\
57.8 \\
62.5\end{array}$ & $\begin{array}{l}42.70 \\
43.30 \\
44.10 \\
44.70 \\
45.40\end{array}$ & $\begin{array}{l}433.34 \\
443.84 \\
456.16 \\
462.04 \\
466.52\end{array}$ & $\begin{array}{l}.003 \\
.004 \\
.004 \\
.004 \\
.003\end{array}$ & $\begin{array}{l}-- \\
-- \\
-- \\
--\end{array}$ & $\begin{array}{l}.204 \\
.206 \\
.210 \\
.212 \\
.214\end{array}$ \\
\hline $\begin{array}{l}P-49 \\
P-48 \\
P-47 \\
P-46 \\
P-45\end{array}$ & $\begin{array}{l}\text { Mudstone, phosphatic } \\
\text { Mudstone, } \\
\text { Mudstone, fos. col. no. 48-JES-i } 76 \\
\text { Phosphate rock and phosphatic mudstone } \\
\text { Mudstone, phosphatic }\end{array}$ & $\begin{array}{l}\text { DFD- } 1753 \\
\text { DFD- } 1752 \\
\text { DFD- } 1751 \\
\text { WOM-1810 } \\
\text { WOM-1809 }\end{array}$ & $\begin{array}{l}1.0 \\
1.1 \\
0.7 \\
0.7 \\
0.7\end{array}$ & $\begin{array}{r}7.9 \\
5.1 \\
5.5 \\
24.6 \\
8.4\end{array}$ & $\begin{array}{l}\because- \\
\because- \\
\because- \\
-\end{array}$ & $\begin{array}{l}-- \\
\because \\
\because \\
-\end{array}$ & $\begin{array}{l}- \\
- \\
- \\
-\end{array}$ & $\begin{array}{l}64.5 \\
74.8 \\
72.8 \\
26.0 \\
55.6\end{array}$ & $\begin{array}{l}46.40 \\
47.50 \\
48.20 \\
48.90 \\
49.60\end{array}$ & $\begin{array}{l}474.42 \\
480.03 \\
483.88 \\
501.10 \\
506.98\end{array}$ & $\begin{array}{l}.004 \\
.002 \\
.003 \\
.005 \\
.005\end{array}$ & $\begin{array}{l}-- \\
-- \\
-- \\
.004 \\
.003\end{array}$ & $\begin{array}{l}.218 \\
.220 \\
.222 \\
.226 \\
.229\end{array}$ \\
\hline \multirow[t]{2}{*}{$P-44$} & Mudstone & WOM-1808 & 0.7 & 5.1 & -- & -- & -- & 71.1 & 50.30 & 510.55 & .003 & -- & .232 \\
\hline & \multicolumn{12}{|c|}{$\begin{array}{l}\text { Many of the beds from P-27 to P-43 show prominent shearing that probably represents faulting and accounts for the abnormally small } \\
\text { thickness of the phosphatic shale member at this locality. }\end{array}$} & \\
\hline $\begin{array}{l}P-43 \\
P-42 \\
P-41 \\
P-40\end{array}$ & $\begin{array}{l}\text { Phosphate rock } \\
\text { Mudstone, phosphatic } \\
\text { Mudstone } \\
\text { Mudstone }\end{array}$ & $\begin{array}{l}\text { WOM-1807 } \\
\text { WOM-1806 } \\
\text { WOM-1805 } \\
\text { WOM-1804 }\end{array}$ & $\begin{array}{l}0.4 \\
0.7 \\
0.8 \\
2.2\end{array}$ & $\begin{aligned} 25.8 \\
13.7 \\
1.2 \\
1.6\end{aligned}$ & $\begin{array}{l}\because- \\
\because \\
\because\end{array}$ & $\begin{array}{l}-- \\
- \\
--\end{array}$ & $\begin{array}{l}\because- \\
\because- \\
-\end{array}$ & $\begin{array}{l}19.5 \\
53.1 \\
84.3 \\
83.8\end{array}$ & $\begin{array}{l}50.70 \\
51.40 \\
52.20 \\
54.40\end{array}$ & $\begin{array}{l}520.87 \\
530.46 \\
531.42 \\
534.94\end{array}$ & $\begin{array}{l}.005 \\
.004 \\
.002 \\
.002\end{array}$ & $\begin{array}{l}.004 \\
-- \\
--\end{array}$ & $\begin{array}{l}.234 \\
.236 \\
.238 \\
.242\end{array}$ \\
\hline $\begin{array}{l}P-39 \\
P-38 \\
P-37 \\
P-36 \\
P-35\end{array}$ & $\begin{array}{l}\text { Mudstone } \\
\text { Mudstone, phosphatic } \\
\text { Mudstone } \\
\text { Mudstone, calcareous } \\
\text { Phosphate rock }\end{array}$ & $\begin{array}{l}\text { WOM-1803 } \\
\text { WOM-1802 } \\
\text { WOM-1801 } \\
\text { WOM-1800 } \\
\text { WOM-1799 }\end{array}$ & $\begin{array}{l}0.6 \\
1.5 \\
2.8 ? \\
0.7 \\
0.5\end{array}$ & $\begin{array}{r}0.9 \\
11.9 \\
4.7 \\
7.3 \\
27.4\end{array}$ & $\begin{array}{l}-- \\
\because- \\
\because-\end{array}$ & $\begin{array}{l}-- \\
-- \\
-- \\
--\end{array}$ & $\begin{array}{l}- \\
\because- \\
-- \\
-\end{array}$ & $\begin{array}{l}83.3 \\
60.3 \\
73.0 \\
58.2 \\
19.8\end{array}$ & $\begin{array}{l}55.00 \\
56.50 \\
59.30 \\
60.00 \\
60.50\end{array}$ & $\begin{array}{l}535.48 \\
552.13 \\
565.29 \\
570.40 \\
584.10\end{array}$ & $\begin{array}{l}.003 \\
.003 \\
.003 \\
.003 \\
.005\end{array}$ & $\begin{array}{l}-- \\
-- \\
-- \\
.004\end{array}$ & $\begin{array}{l}.244 \\
.249 \\
.257 \\
.259 \\
.262\end{array}$ \\
\hline \multirow[t]{2}{*}{$\begin{array}{l}P-34 \\
P-33 \\
P-32 \\
P-31 \\
P-30\end{array}$} & \multirow[t]{2}{*}{$\begin{array}{l}\text { Mudstone, phosphatic } \\
\text { Phosphate, rock, argilaceous } \\
\text { Mudstone, calcarrous } \\
\text { Mudstone, phosphatic } \\
\text { Mudatone, phosphatic, tos. col. no. } \\
\text { 18-JES-175? }\end{array}$} & $\begin{array}{l}\text { WOM-1798 } \\
\text { WOM-1797 } \\
\text { WOM-1796 } \\
\text { WOM-1795 }\end{array}$ & $\begin{array}{l}1.0 \\
0.6 \\
1.0 \\
0.5\end{array}$ & $\begin{array}{r}9.5 \\
18.1 \\
2.8 \\
11.1\end{array}$ & $\begin{array}{l}\because- \\
\because \\
\because\end{array}$ & $\begin{array}{l}-- \\
\because \\
\because-\end{array}$ & $\begin{array}{l}-: \\
\because- \\
z\end{array}$ & $\begin{array}{l}61.3 \\
37.7 \\
58.5 \\
51.9\end{array}$ & $\begin{array}{l}61.50 \\
62.10 \\
63.10 \\
63.60\end{array}$ & $\begin{array}{l}593.60 \\
604.46 \\
607.26 \\
612.81\end{array}$ & $\begin{array}{l}.003 \\
.005 \\
.002 \\
.004\end{array}$ & $\begin{array}{l}-- \\
.003 \\
\cdots \\
--\end{array}$ & $\begin{array}{l}.265 \\
.268 \\
.270 \\
.272\end{array}$ \\
\hline & & WOM-1794 & 1.3 & 10.4 & -- & -- & -- & 49.7 & 64.90 & 626.33 & .004 & -- & .277 \\
\hline
\end{tabular}

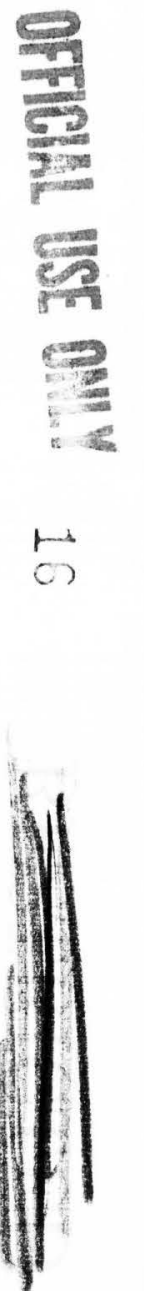




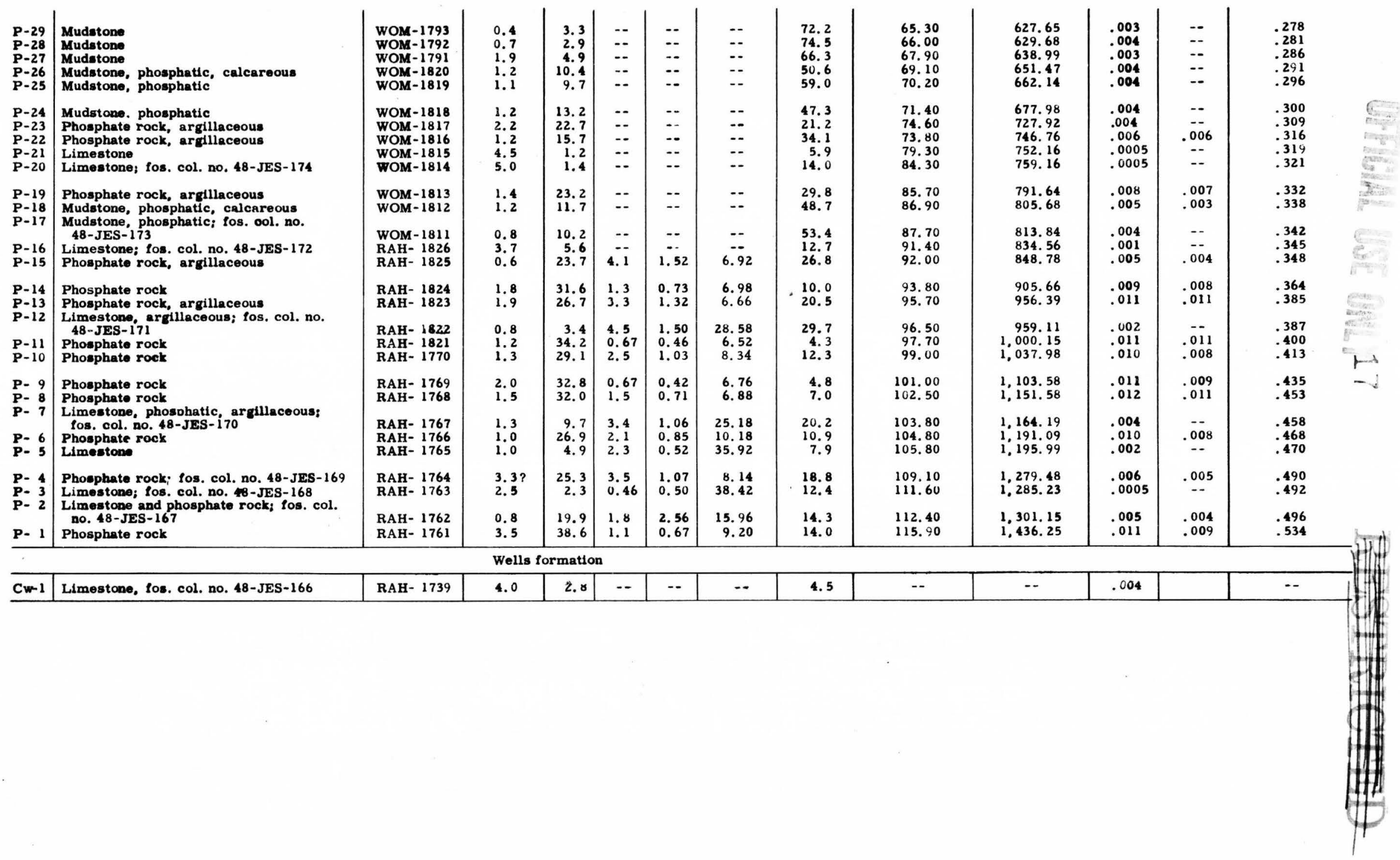


MABIE CANYON, DAHO. LOT NO. 1210.

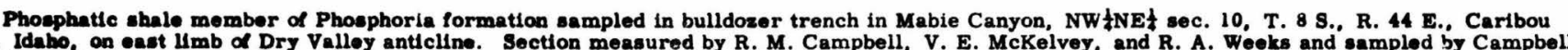
and wooke in Soptember and October 1947. Samples analyzeu by U. S. Bureau of Mines Laboratory, Albany, Oregon.

\begin{tabular}{|c|c|}
\hline $\begin{array}{l}\text { Bod } \\
\text { no. }\end{array}$ & Rook description \\
\hline $\begin{array}{l}\mathbf{R}: \\
\mathbf{R}: \\
\mathbf{R}: \\
\mathbf{R}: \\
\mathbf{R}:\end{array}$ & $\begin{array}{l}\text { Chort } \\
\text { Limestone, argillaceous } \\
\text { Chert } \\
\text { Mudstone, calcafeous; fos. col. } \\
\text { no. 17-HW-259l } \\
\text { Mudetono, calcareous and chert }\end{array}$ \\
\hline
\end{tabular}

\begin{tabular}{c|c}
$\begin{array}{c}\text { Sample } \\
\text { no. }\end{array}$ & This \\
\hline
\end{tabular}

\begin{tabular}{|c|c|c|c|c|c|c|}
\multirow{2}{*}{$\begin{array}{c}\text { Thickness } \\
\text { (feet) }\end{array}$} & \multicolumn{5}{|c|}{ Chemical analyses (percent) } \\
\cline { 2 - 7 } & $\mathrm{P}_{2} \mathrm{O}_{5}$ & $\mathrm{Al}_{2} \mathrm{O}_{3}$ & $\mathrm{Fe}_{2} \mathrm{O}_{3}$ & $\mathrm{~F}$ & $\begin{array}{c}\text { Loss on } \\
\text { Ignition }\end{array}$ & $\begin{array}{c}\text { Acdd } \\
\text { insoluble }\end{array}$ \\
\cline { 2 - 7 }
\end{tabular}

Cumulative
thickness thickness
(feet)

Rex momber of Phosphoria formation-basal bods oaly

\begin{tabular}{|c|c|c|c|c|c|c|c|c|c|c|c|c|}
\hline $\begin{array}{l}\text { JEM-470-47 } \\
\text { VMM-469-47 } \\
\mathrm{VEM}-468-47\end{array}$ & $\begin{array}{l}3.8 \\
1.2 \\
1.7\end{array}$ & $\begin{array}{l}0.5 \\
0.3 \\
0.6\end{array}$ & $\begin{array}{l}1.1 \\
0.7 \\
0.79\end{array}$ & $\begin{array}{l}3.5 \\
1.8 \\
3.8\end{array}$ & $\begin{array}{l}-- \\
\because-\end{array}$ & $\begin{array}{r}9.6 \\
32.0 \\
6.7\end{array}$ & $\begin{array}{l}73.3 \\
25.9 \\
78.6\end{array}$ & $\begin{array}{l}3.8 \\
5.0 \\
6.7\end{array}$ & $\begin{array}{l}1.90 \\
2.26 \\
3.28\end{array}$ & $\begin{array}{l}.0005 \\
.0005 \\
.0005\end{array}$ & $\begin{array}{l}-- \\
--\end{array}$ & $\begin{array}{l}.002 \\
.002 \\
.003\end{array}$ \\
\hline $\begin{array}{l}\text { IEM-467-47 } \\
\text { EMM-466-472 }\end{array}$ & $\begin{array}{l}0.35 \\
1.0\end{array}$ & 0.5 & 1.3 & 1.5 & $=$ & 21.2 & 51. 1 & $\begin{array}{l}7.05 \\
8.05\end{array}$ & $\begin{array}{l}3.46 \\
-.\end{array}$ & .0005 & $=$ & .004 \\
\hline
\end{tabular}

$$
\text { Phosphatic shale member of Phosphoria formation }
$$

\begin{tabular}{|c|c|c|}
\hline & $\begin{array}{l}-170 \\
-169\end{array}$ & $\begin{array}{l}\text { Mudatone, calcareous } \\
\text { Phosphate rock, argelllaceous, } \\
\text { calcareous; fos. col. no. } \\
\text { 47-HW-258 }\end{array}$ \\
\hline$P$ & -168 & $\begin{array}{l}\text { mudstone, calcareous; fos. col. } \\
\text { no. } 47-\mathrm{HW}-257\end{array}$ \\
\hline & $\begin{array}{l}-167 \\
-166\end{array}$ & $\begin{array}{l}\text { Mudstone, calcareous } \\
\text { Mudatean }\end{array}$ \\
\hline & -- & $\begin{array}{l}\text { Limestone lons, argillaceous, } \\
\text { in top of bed } P-165\end{array}$ \\
\hline P & $\begin{array}{l}-165 \\
-164 \\
-163 \\
-162\end{array}$ & $\begin{array}{l}\text { Mudstone, calcareous } \\
\text { Mudatone, calcareous } \\
\text { Mudetone, celcareous } \\
\text { Mudatone, calcareous }\end{array}$ \\
\hline $\mathbf{P}$ & -161 & Limpotono; los. col. no. \\
\hline $\mathbf{P}$ & -160 & Mudatone, calcareous; fos. col. \\
\hline & -159 & $\begin{array}{l}\text { Mudstons, calcareous; fos. col. } \\
\text { no. 47-EW-254 }\end{array}$ \\
\hline $\boldsymbol{P}$ & -158 & arefllanosous, conta \\
\hline $\mathbf{P}$ & -157 & Mudatone, ealcareous \\
\hline & $\begin{array}{l}-156 \\
-155\end{array}$ & $\begin{array}{l}\text { Phosphate roek, arglllaceous, } \\
\text { madewoos; fos. col, na } \\
\text { 17-tW-253 }\end{array}$ \\
\hline
\end{tabular}

\begin{tabular}{|c|c|c|c|c|c|}
\hline VEM $-465-47$ & 0.7 & 5.1 & 4.0 & 1.5 & 0.53 \\
\hline VEM $-464-47$ & 0.6 & 20.1 & 4.0 & 1.3 & -. \\
\hline $\begin{array}{l}\text { VEM-463-47 } \\
\text { VEM-462-47 } \\
\text { VEM }-46]-47\end{array}$ & $\begin{array}{l}1.9 \\
2.1\end{array}$ & $\begin{array}{l}2.9 \\
4.7 \\
0.6\end{array}$ & $\begin{array}{r}9.9 \\
9.7 \\
11.7\end{array}$ & $\begin{array}{l}\text { 3. } 0 \\
\text { 3. } 2 \\
\text { 3.1 }\end{array}$ & $=$ \\
\hline VEM-461-47 & 1.3 & 0.6 & 11.1 & 3.1 & \\
\hline $\begin{array}{l}\text { VEM-460-47 } \\
\text { VEM-459-47 } \\
\text { VEM-458-47 } \\
\text { VEM-457-47 } \\
\text { VEM-456-47 }\end{array}$ & $\begin{array}{c}(0.0-0.5) \\
1.2 \\
2.4 \\
1.9 \\
2.4\end{array}$ & $\begin{array}{l}0.7 \\
2.6 \\
0.4 \\
0.4 \\
1.3\end{array}$ & $\begin{array}{r}3.6 \\
11.6 \\
7.4 \\
9.4 \\
11.0\end{array}$ & $\begin{array}{l}1.3 \\
3.7 \\
2.4 \\
3.1 \\
3.6\end{array}$ & $\begin{array}{l}- \\
\cdots \\
\cdots \\
\cdots\end{array}$ \\
\hline VEM $-455-47$ & 0.7 & 0.6 & 2.2 & 0.8 & -- \\
\hline VEM $-454-47$ & 1.8 & 4.0 & 10.6 & 3.0 & -- \\
\hline VEM-453-47 & 1.8 & 1.5 & 9.2 & 2.7 & $\cdots$ \\
\hline $\begin{array}{l}\text { VEM-452-47 } \\
\text { VEM-451-47 }\end{array}$ & $\begin{array}{l}0.9 \\
0.7\end{array}$ & $\begin{array}{l}6.3 \\
3.0\end{array}$ & $\begin{array}{l}1.3 \\
9.2\end{array}$ & $\begin{array}{l}3.3 \\
3.2\end{array}$ & $=$ \\
\hline VEM $-450-47$ & 0.5 & 19.9 & 3.1 & 1.8 & - \\
\hline VEM-449-47 & 2.4 & 3.1 & 10.7 & 3.1 & \\
\hline
\end{tabular}

\begin{tabular}{r|r|}
13.8 & 60.1 \\
12.2 & 26.6 \\
9.9 & 69.4 \\
12.7 & 61.7 \\
8.1 & 76.6 \\
& \\
34.5 & 22.5 \\
8.7 & 69.0 \\
19.8 & 52.5 \\
12.0 & 67.5 \\
11.0 & 66.0 \\
& \\
37.7 & 14.0 \\
10.7 & 62.4 \\
12.7 & 64.8 \\
27.5 & 24.3 \\
8.3 & 68.7 \\
9.0 & 28.5 \\
8.9 & 69.9 \\
&
\end{tabular}

\begin{tabular}{|l|}
\hline 0.7 \\
1.3 \\
3.2 \\
5.3 \\
6.6 \\
\hline.- \\
7.8 \\
10.2 \\
12.1 \\
14.5 \\
15.2 \\
17.0 \\
18.8 \\
19.7 \\
20.4 \\
20.9 \\
23.3
\end{tabular}

3.57
15.63
21.14
31.01
31.79

34.91
35.87
36.63
39.75
40.17
47.37
50.07
55.73
57.84
67.79
75.23

\begin{tabular}{c|c|}
.004 & -- \\
.009 & .007 \\
.003 & -- \\
.005 & .004 \\
.002 & -- \\
.001 & -- \\
.003 & -- \\
.001 & - \\
.002 & - \\
& -- \\
.001 & -- \\
.003 & -- \\
.002 & -- \\
.002 & -- \\
.002 & -- \\
.007 & .003 \\
.003 & -- \\
&
\end{tabular}

.003

.008

.014

.024

031

34. 91

35.87

39.75

40.17

47. 37

57. 8

67.79

75.23

Branch.

rcent eU 


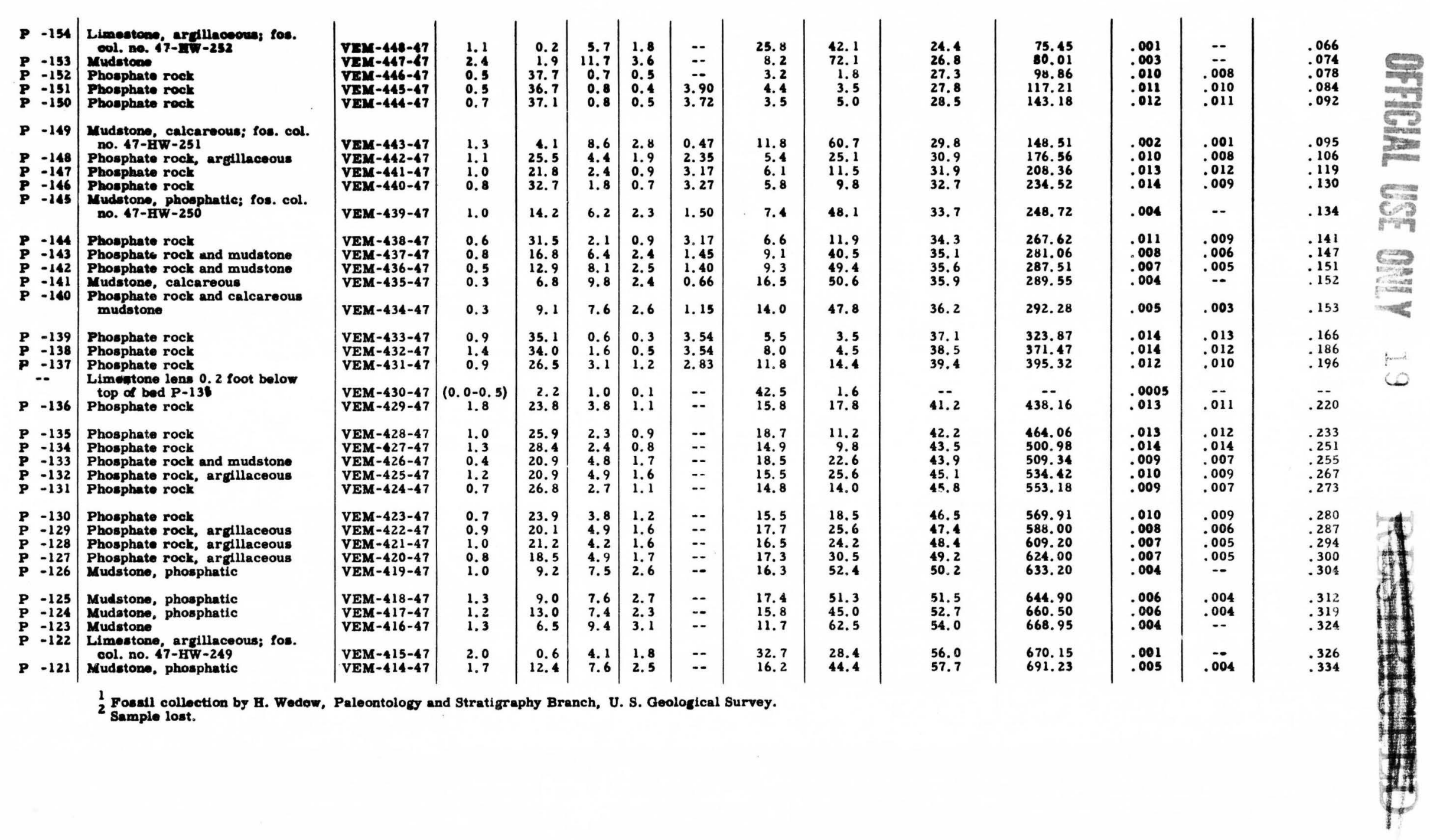




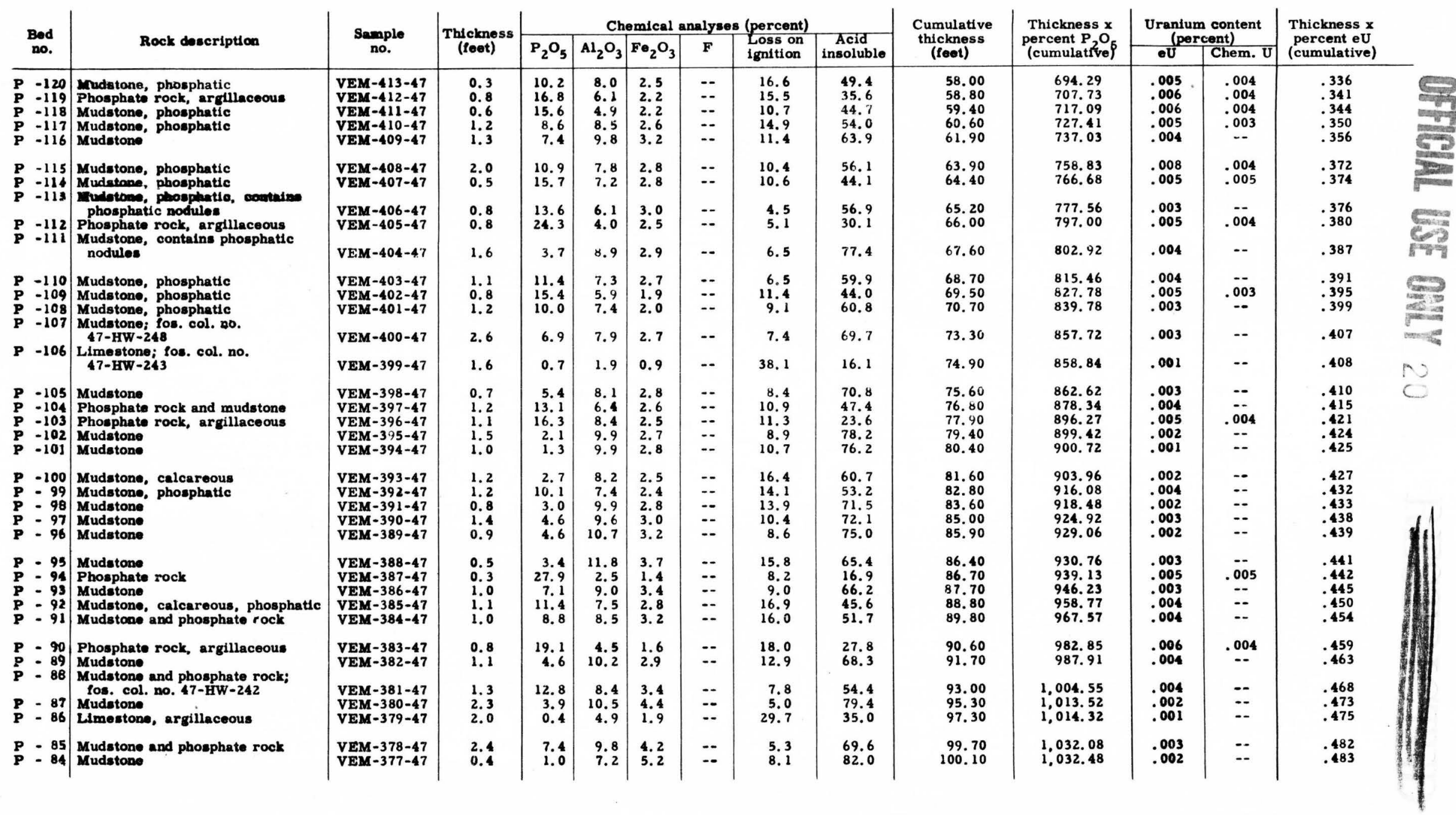




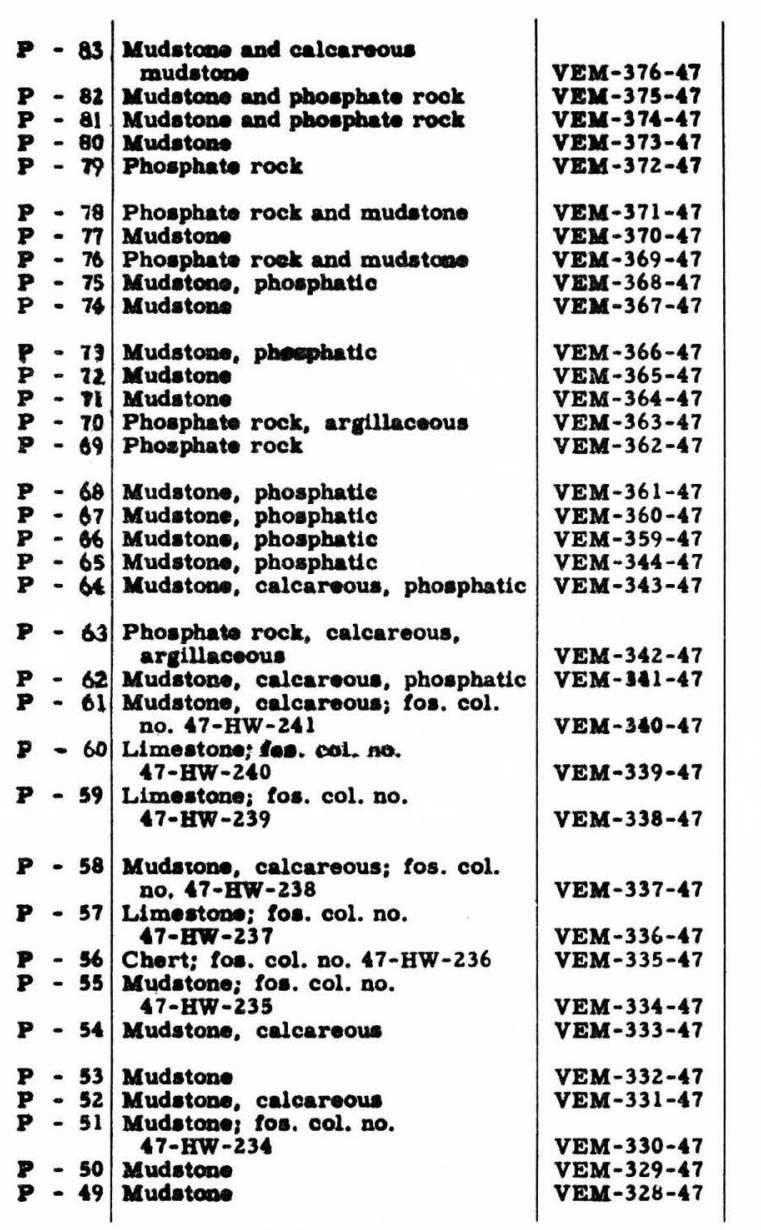

\begin{tabular}{|c|c|c|c|c|c|}
\hline $\begin{array}{l}2.8 \\
0.5 \\
2.9 \\
1.0 \\
1.0\end{array}$ & $\begin{array}{r}0.4 \\
14.7 \\
9.8 \\
1.7 \\
32.2\end{array}$ & $\begin{array}{r}6.8 \\
7.9 \\
9.9 \\
12.0 \\
2.4\end{array}$ & $\begin{array}{l}2.5 \\
3.3 \\
4.7 \\
3.8 \\
2.4\end{array}$ & $\begin{array}{l}\because- \\
\therefore \\
\therefore\end{array}$ & $\begin{array}{r}24.2 \\
5.5 \\
5.3 \\
5.4 \\
3.3\end{array}$ \\
\hline $\begin{array}{l}0.7 \\
1.4 \\
0.9 \\
0.4 \\
1.1\end{array}$ & $\begin{array}{r}21.4 \\
1.6 \\
22.5 \\
9.6 \\
3.5\end{array}$ & $\begin{array}{r}5.4 \\
11.5 \\
5.5 \\
10.5 \\
11.7\end{array}$ & $\begin{array}{l}4.9 \\
5.2 \\
3.5 \\
3.6 \\
3.5\end{array}$ & $\begin{array}{l}\because \\
\therefore \\
\therefore \\
\therefore\end{array}$ & $\begin{array}{r}5.2 \\
6.7 \\
6.4 \\
6.8 \\
6.7\end{array}$ \\
\hline $\begin{array}{l}0.9 \\
1.3 \\
0.8 \\
0.4 \\
0.7\end{array}$ & $\begin{array}{r}14.0 \\
0.9 \\
4.8 \\
17.1 \\
24.3\end{array}$ & $\begin{array}{r}8.0 \\
4.0 \\
11.0 \\
5.3 \\
3.3\end{array}$ & $\begin{array}{l}3.0 \\
3.6 \\
3.5 \\
2.4 \\
1.4\end{array}$ & $\begin{array}{l}-- \\
\therefore- \\
\therefore-\end{array}$ & $\begin{array}{r}11.8 \\
8.6 \\
8.2 \\
19.7 \\
17.9\end{array}$ \\
\hline $\begin{array}{l}0.8 \\
1.0 \\
0.8 \\
0.1 \\
1.8\end{array}$ & $\begin{array}{r}11.1 \\
13.3 \\
8.2 \\
9.1 \\
10.1\end{array}$ & $\begin{array}{r}8.7 \\
7.9 \\
10.6 \\
9.6 \\
7.7\end{array}$ & $\begin{array}{l}2.7 \\
2.7 \\
3.5 \\
3.3 \\
2.9\end{array}$ & 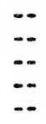 & $\begin{array}{l}14.0 \\
17.2 \\
14.5 \\
25.2 \\
21.5\end{array}$ \\
\hline $\begin{array}{l}1.2 \\
1.6\end{array}$ & $\begin{array}{r}13.7 \\
7.9\end{array}$ & $\begin{array}{l}5.3 \\
8.0\end{array}$ & $\begin{array}{l}2.2 \\
2.7\end{array}$ & -- & $\begin{array}{l}19.2 \\
18.9\end{array}$ \\
\hline 0.4 & 5.7 & 10.6 & 3.3 & - & 15.2 \\
\hline 0.5 & 0.3 & 2.0 & 0.8 & -- & 40.6 \\
\hline 1.5 & 0.3 & 1.4 & 0.4 & -- & 40.8 \\
\hline 1.1 & 1.8 & 11.9 & 3.4 & $\cdots$ & 13.7 \\
\hline $\begin{array}{l}3.6 \\
0.3\end{array}$ & $\begin{array}{l}0.3 \\
1.0\end{array}$ & $\begin{array}{l}1.9 \\
2.4\end{array}$ & $\begin{array}{l}0.5 \\
3.5\end{array}$ & 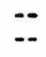 & $\begin{array}{r}38.5 \\
5.0\end{array}$ \\
\hline $\begin{array}{l}1.0 \\
1.5\end{array}$ & $\begin{array}{l}1.8 \\
2.9\end{array}$ & $\begin{array}{r}7.6 \\
11.7\end{array}$ & $\begin{array}{l}2.6 \\
3.3\end{array}$ & 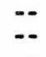 & $\begin{array}{r}6.9 \\
12.1\end{array}$ \\
\hline $\begin{array}{l}1.7 \\
1.6\end{array}$ & $\begin{array}{l}1.7 \\
4.1\end{array}$ & $\begin{array}{r}11.7 \\
9.2\end{array}$ & $\begin{array}{l}3.5 \\
2.9\end{array}$ & $=$ & $\begin{array}{l}10.0 \\
19.0\end{array}$ \\
\hline $\begin{array}{l}1.2 \\
1.6 \\
1.7\end{array}$ & $\begin{array}{l}3.7 \\
6.8 \\
1.6\end{array}$ & $\begin{array}{l}10.3 \\
11.1 \\
11.4\end{array}$ & $\begin{array}{l}3.1 \\
3.4 \\
3.6\end{array}$ & $\begin{array}{l}\because- \\
\because-\end{array}$ & $\begin{array}{l}9.1 \\
9.2 \\
8.5\end{array}$ \\
\hline
\end{tabular}

\begin{tabular}{l|l} 
& \\
47.4 & 102.90 \\
30.9 & 103.40 \\
63.9 & 106.30 \\
84.7 & 107.30 \\
12.0 & 108.30 \\
33.3 & 109.00 \\
81.8 & 110.40 \\
32.0 & 111.30 \\
59.6 & 11.70 \\
78.7 & 112.80 \\
45.9 & 113.70 \\
84.1 & 115.00 \\
74.6 & 115.80 \\
26.7 & 116.20 \\
14.9 & 116.90 \\
50.7 & 117.70 \\
40.7 & 118.70 \\
55.0 & 119.90 \\
36.8 & 119.90 \\
36.7 & 121.70 \\
& \\
27.6 & 122.90 \\
40.7 & 124.50 \\
52.3 & 124.90 \\
11.9 & 125.40 \\
11.3 & 126.90 \\
& \\
65.6 & 128.00 \\
17.4 & 131.60 \\
85.1 & 131.90 \\
81.1 & 132.90 \\
68.9 & 134.40 \\
76.6 & 136.10 \\
48.6 & 137.70 \\
72.6 & 138.90 \\
64.3 & 140.50 \\
81.0 & 142.20 \\
& \\
& \\
\hline 1.9 &
\end{tabular}

\begin{tabular}{|c|c|c|}
\hline 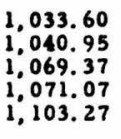 & $\begin{array}{l}: 002 \\
: 004 \\
: 003 \\
: 002 \\
0.005\end{array}$ & $\begin{array}{l}\because: \\
\because \ddot{0} \\
.004\end{array}$ \\
\hline 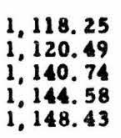 & $\begin{array}{l}.005 \\
.002 \\
0.005 \\
.005 \\
.002\end{array}$ & $\begin{array}{l}.004 \\
.004 \\
.004 \\
.04\end{array}$ \\
\hline 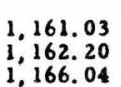 & $\begin{array}{l}.005 \\
: 003 \\
.003\end{array}$ & .004 \\
\hline $\begin{array}{l}1,172.88 \\
1,189.89\end{array}$ & : & $\begin{array}{l}.003 \\
.012\end{array}$ \\
\hline $\begin{array}{l}1,198.77 \\
1,212.07 \\
1,212.03\end{array}$ & $\begin{array}{l}.008 \\
: 0.068 \\
.004\end{array}$ & $\begin{array}{r}.006 \\
.004\end{array}$ \\
\hline $\begin{array}{l}1,222.27 \\
1,240.45\end{array}$ & :005 & $\begin{array}{r}.003 \\
.001\end{array}$ \\
\hline $\begin{array}{l}1,256.89 \\
1,269.53\end{array}$ & :0033 & $\because$ \\
\hline $1,271.81$ & . 003 & .001 \\
\hline 1.271 .96 & .0005 & -- \\
\hline $1,272.41$ & .0005 & -- \\
\hline 1.274. 39 & .002 & $-\cdot$ \\
\hline $\begin{array}{l}1,2755.47 \\
1,275.77\end{array}$ & : 00005 & $\because$ \\
\hline $\begin{array}{l}1,277.57 \\
1,281.92\end{array}$ & :002 & $\because$ \\
\hline $\begin{array}{l}1,284.81 \\
1 ; 291.37\end{array}$ & $\begin{array}{l}.0022 \\
.002\end{array}$ & $\because$ \\
\hline $\begin{array}{l}1,295.81 \\
1 \\
1,306 \\
1,309.41\end{array}$ & $\begin{array}{l}.002 \\
: 0003 \\
.001\end{array}$ & $\because$ \\
\hline
\end{tabular}

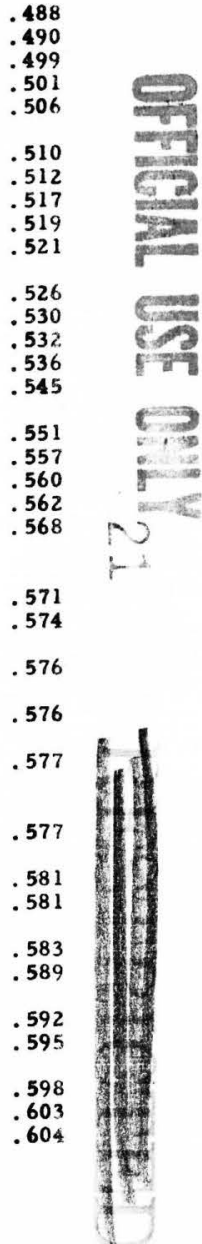




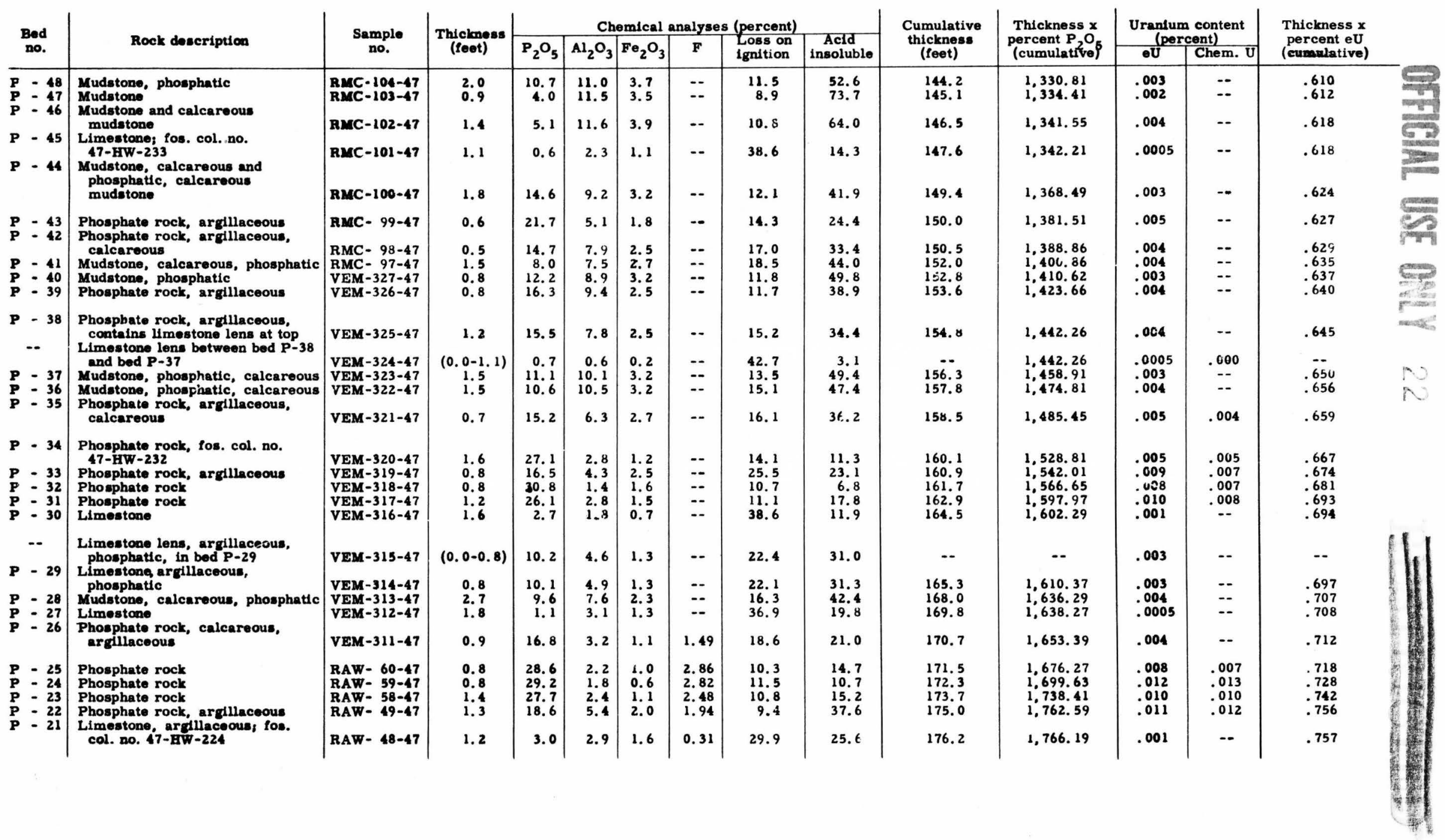




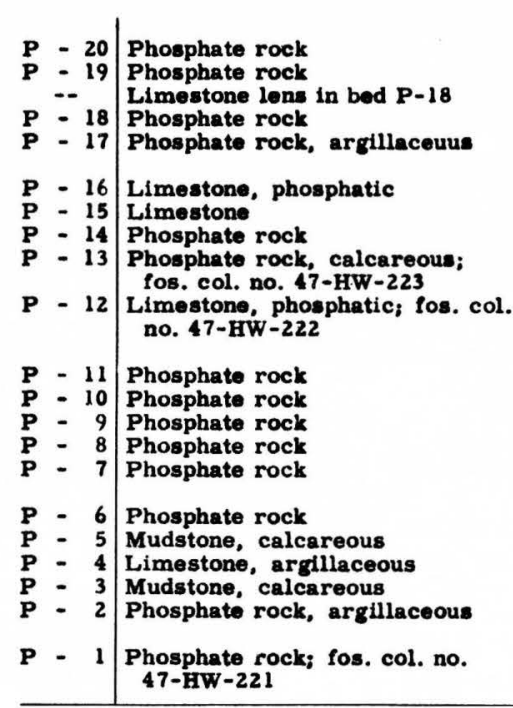

\begin{tabular}{|l|} 
RAW- 47-47 \\
RAW- 46-47 \\
RAW- 45-47 \\
RAW- $44-47$ \\
RAW- 43-47 \\
RAW- 42-47 \\
RAW- 41-47 \\
RAW- 40-47 \\
RAW- 39-47 \\
RAW- 38-47 \\
RAW- 37-47 \\
RAW- 36-37 \\
RAW- 35-47 \\
RAW- 34-47 \\
RAW- 33-47 \\
RAW- 32-47 \\
RAW- 31-47 \\
RAW- 30-47 \\
RAW- 29-47 \\
RAW- 28-47 \\
RAW- 61-47 \\
RAW-
\end{tabular}

\begin{tabular}{|c|c|c|c|c|}
\hline $\begin{array}{c}0.9 \\
2.1 \\
(0.5) \\
2.0 \\
1.7\end{array}$ & $\begin{array}{r}32.5 \\
28.7 \\
1.3 \\
26.2 \\
23.1\end{array}$ & $\begin{array}{l}1.3 \\
2.5 \\
0.4 \\
2.5 \\
4.1\end{array}$ & $\begin{array}{l}0.8 \\
0.9 \\
0.2 \\
1.3 \\
1.3\end{array}$ & $\begin{array}{l}3.30 \\
2.47 \\
.2 .50 \\
2.16\end{array}$ \\
\hline $\begin{array}{l}0.9 \\
0.8 \\
1.5\end{array}$ & $\begin{array}{r}8.9 \\
2.0 \\
26.9\end{array}$ & $\begin{array}{l}1.3 \\
2.5 \\
3.2\end{array}$ & $\begin{array}{l}0.7 \\
0.7 \\
0.7\end{array}$ & $\begin{array}{l}0.86 \\
2.55\end{array}$ \\
\hline 1.4 & 20.2 & 2.8 & 1.0 & 2.04 \\
\hline 1.5 & 10.5 & 0.8 & 1.2 & 1.06 \\
\hline $\begin{array}{l}1.0 \\
1.1 \\
1.2 \\
1.1 \\
0.6\end{array}$ & $\begin{array}{l}32.6 \\
32.4 \\
32.0 \\
32.4 \\
30.1\end{array}$ & $\begin{array}{l}1.2 \\
0.94 \\
1.1 \\
0.98 \\
1.4\end{array}$ & $\begin{array}{l}0.4 \\
0.5 \\
0.8 \\
0.6 \\
0.9\end{array}$ & $\begin{array}{l}2.67 \\
3.21 \\
3.17 \\
3.24 \\
3.07\end{array}$ \\
\hline $\begin{array}{l}1.4 \\
1.2 \\
2.6 \\
2.3 \\
0.2\end{array}$ & $\begin{array}{r}31.0 \\
3.8 \\
0.4 \\
0.7 \\
20.8\end{array}$ & $\begin{array}{l}1.4 \\
9.8 \\
4.2 \\
8.0 \\
5.6\end{array}$ & $\begin{array}{l}0.8 \\
3.5 \\
2.0 \\
2.3 \\
1.8\end{array}$ & $\begin{array}{c}3.17 \\
-\therefore \\
-- \\
--\end{array}$ \\
\hline 0.4 & 29.6 & 1.1 & 0.6 & 2. 90 \\
\hline
\end{tabular}

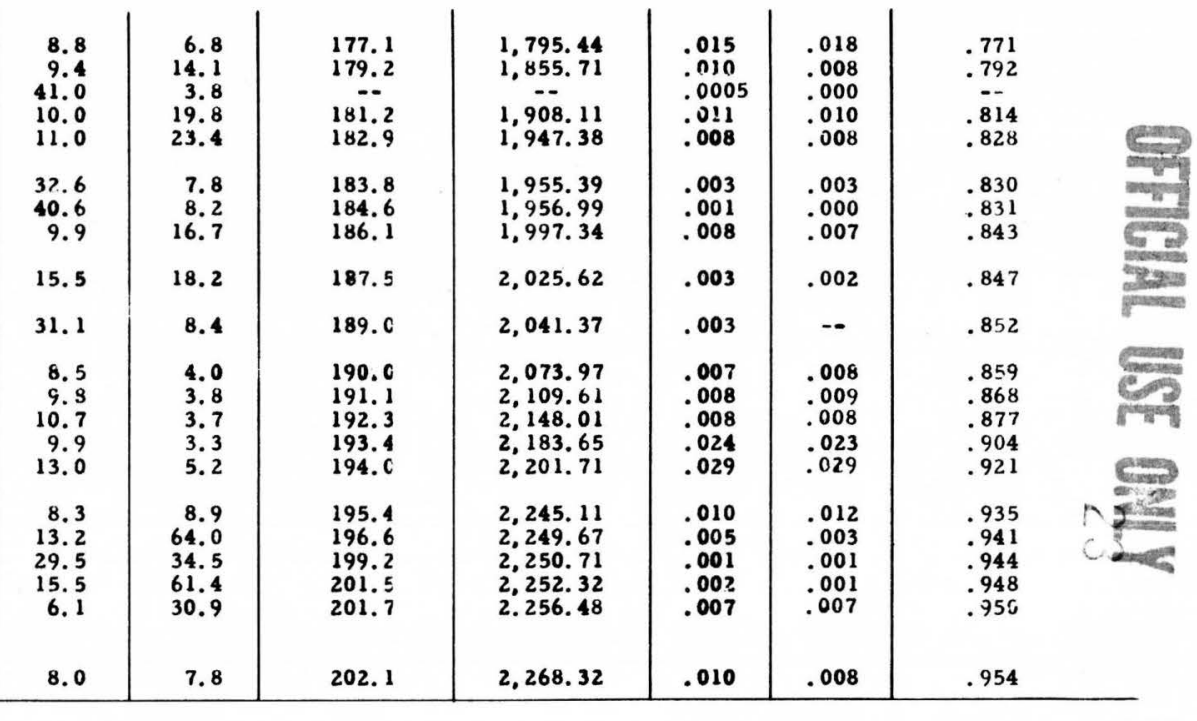

CW- 1 Limestone; fos. col. no.

Cw- 2 Phosphate rock

\begin{tabular}{l|l|l|l|l|l}
-- & 4.6 & -- & -- & -- & -- \\
-- & 0.2 & -- & - & -- & -
\end{tabular}

$-$

$-\cdot$

-
--

$--$

\begin{tabular}{|c|c|c} 
& & - \\
-- & -- & -- \\
\hline
\end{tabular}


SPECTROGRAPHIC ANALYSES-MABIE CANYON, IDAHO. LOT NO. 1210.

Semi-quantitative analyses of samples of the Phosphoria formation, Mabie Canyon, Idaho (see immediately preceding pages for location of section, thickness and description of strata, and chemical analyses of samples), made by U. S. Bureau of Mines Laboratory, Albany, Oregon. In addition to the elements listed in the table below, $\mathrm{Sb}, \mathrm{As}, \mathrm{Ba}, \mathrm{Be}, \mathrm{Bi}, \mathrm{Cd}, \mathrm{Cb}, \mathrm{Ga}, \mathrm{Ge}, \mathrm{Au}, \mathrm{In}, \mathrm{Pb}, \mathrm{Li}, \mathrm{Hg}, \mathrm{Pt}, \mathrm{Ta}, \mathrm{Sn}, \mathrm{and} \mathrm{W}$ were looked for in all samples but were not detected.

Explanation of symbols

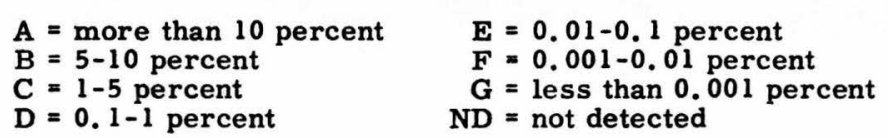

\begin{tabular}{|c|c|c|c|c|c|c|c|c|c|c|c|c|c|c|c|c|c|c|c|c|c|}
\hline & $\begin{array}{l}\text { ed } \\
\text { ao. }\end{array}$ & Sample no. & Al & B & $\mathrm{Ca}$ & $\mathrm{Cr}$ & Co & $\mathrm{Cu}$ & $\mathrm{Fe}$ & $\mathbf{M g}$ & $\mathbf{M n}$ & Mo & $\mathrm{Ni}$ & $\mathbf{S i}$ & Ag & $\mathrm{Na}$ & $\mathbf{S r}$ & $\mathbf{T i}$ & V & $\mathrm{Zn}_{\mathrm{n}}$ & $\mathbf{Z r}$ \\
\hline $\begin{array}{l}\mathbf{R} \\
\mathbf{R} \\
\mathbf{R} \\
\mathbf{R} \\
\mathbf{R}\end{array}$ & $\begin{array}{l}-\quad 5 \\
-\quad 4 \\
-\quad 3 \\
-\quad 2 \\
-\quad 1\end{array}$ & $\begin{array}{l}\text { VEM-470-47 } \\
\text { VEM-469-47 } \\
\text { VEM-468-47 } \\
\text { VEM-467-47 } \\
\text { VEM-466-47 }\end{array}$ & $\begin{array}{l}\mathbf{C} \\
\mathbf{C} \\
\mathbf{C} \\
\mathbf{C} \\
--\end{array}$ & $\begin{array}{l}\mathbf{F} \\
\mathbf{F} \\
\mathbf{F} \\
\mathbf{F} \\
--\end{array}$ & $\begin{array}{c}\text { C } \\
\mathbf{A} \\
\mathbf{C} \\
\mathbf{A} \\
--\end{array}$ & $\begin{array}{l}\mathbf{E} \\
\mathbf{E} \\
\mathbf{E} \\
\mathbf{E} \\
--\end{array}$ & $\begin{array}{l}\text { ND } \\
\text { ND } \\
\text { ND } \\
\text { ND } \\
--\end{array}$ & $\begin{array}{l}\mathbf{G} \\
\mathbf{G} \\
\mathbf{G} \\
\mathbf{G} \\
--\end{array}$ & $\begin{array}{l}\text { C } \\
\text { C } \\
\text { C } \\
\text { C } \\
--\end{array}$ & $\begin{array}{l}\text { C } \\
\text { C } \\
\text { D } \\
\text { C } \\
--\end{array}$ & $\begin{array}{l}\mathbf{E} \\
\mathbf{E} \\
\mathbf{E} \\
\mathbf{E} \\
--\end{array}$ & $\begin{array}{l}\mathbf{F} \\
\mathbf{F} \\
\mathbf{F} \\
\mathbf{F} \\
--\end{array}$ & $\begin{array}{l}\mathbf{E} \\
\mathbf{E} \\
\mathbf{E} \\
\mathbf{E} \\
--\end{array}$ & $\begin{array}{l}\text { A } \\
\text { A } \\
\text { A } \\
\text { A } \\
--\end{array}$ & $\begin{array}{c}\mathbf{G} \\
\mathbf{G} \\
\mathbf{G} \\
\mathbf{G} \\
--\end{array}$ & $\begin{array}{l}\mathbf{E} \\
\mathbf{E} \\
\mathbf{E} \\
\mathbf{E} \\
--\end{array}$ & $\begin{array}{l}\mathbf{F} \\
\mathbf{F} \\
\mathbf{F} \\
\mathbf{F} \\
--\end{array}$ & $\begin{array}{l}\mathbf{E} \\
\mathbf{E} \\
\mathbf{E} \\
\mathbf{E} \\
--\end{array}$ & $\begin{array}{l}\mathbf{E} \\
\mathbf{E} \\
\mathbf{E} \\
\mathbf{E} \\
--\end{array}$ & $\begin{array}{c}\text { ND } \\
\text { ND } \\
\text { ND } \\
\text { E } \\
--\end{array}$ & $\begin{array}{l}\mathbf{E} \\
\mathbf{E} \\
\mathbf{E} \\
\mathbf{E} \\
--\end{array}$ \\
\hline $\begin{array}{l}\mathbf{P} \\
\mathbf{P} \\
\mathbf{P} \\
\mathbf{P} \\
\mathbf{P}\end{array}$ & $\begin{array}{l}-170 \\
-169 \\
-168 \\
-167 \\
-166\end{array}$ & $\begin{array}{l}\text { VEM-465-47 } \\
\text { VEM-464-47 } \\
\text { VEM-463-47 } \\
\text { VEM-462-47 } \\
\text { VEM-461-47 }\end{array}$ & $\begin{array}{l}\text { C } \\
\text { C } \\
\text { C } \\
\text { C } \\
\text { C }\end{array}$ & $\begin{array}{l}\mathbf{F} \\
\mathbf{F} \\
\mathbf{F} \\
\mathbf{F} \\
\mathbf{F}\end{array}$ & $\begin{array}{l}\text { A } \\
\text { A } \\
\text { C } \\
\text { C } \\
\text { C }\end{array}$ & $\begin{array}{l}\mathbf{E} \\
\mathbf{E} \\
\mathbf{E} \\
\mathbf{E} \\
\mathbf{E}\end{array}$ & $\begin{array}{l}\text { ND } \\
\text { ND } \\
\text { ND } \\
\text { ND } \\
\text { ND }\end{array}$ & $\begin{array}{l}\mathbf{G} \\
\mathbf{G} \\
\mathbf{G} \\
\mathbf{G} \\
\mathbf{G}\end{array}$ & $\begin{array}{l}\text { C } \\
\text { C } \\
\text { C } \\
\text { C } \\
\text { C }\end{array}$ & $\begin{array}{l}\mathrm{D} \\
\mathrm{D} \\
\mathrm{D} \\
\mathrm{D} \\
\mathrm{D}\end{array}$ & $\begin{array}{l}\mathbf{E} \\
\mathbf{E} \\
\mathbf{E} \\
\mathbf{E} \\
\mathbf{E}\end{array}$ & $\begin{array}{l}\mathbf{F} \\
\mathbf{F} \\
\mathbf{F} \\
\mathbf{F} \\
\mathbf{F}\end{array}$ & $\begin{array}{l}\mathbf{E} \\
\mathbf{E} \\
\mathbf{E} \\
\mathbf{E} \\
\mathbf{E}\end{array}$ & $\begin{array}{l}\text { A } \\
\mathbf{A} \\
\mathbf{A} \\
\mathbf{A} \\
\mathbf{A}\end{array}$ & $\begin{array}{l}\mathbf{G} \\
\mathbf{G} \\
\mathbf{G} \\
\mathbf{G} \\
\mathbf{G}\end{array}$ & $\begin{array}{l}\mathbf{E} \\
\mathbf{E} \\
\mathbf{E} \\
\mathbf{D} \\
\mathbf{D}\end{array}$ & $\begin{array}{l}\mathbf{F} \\
\mathbf{E} \\
\mathbf{F} \\
\mathbf{F} \\
\mathbf{F}\end{array}$ & $\begin{array}{l}\mathbf{E} \\
\mathbf{E} \\
\mathbf{E} \\
\mathbf{E} \\
\mathbf{E}\end{array}$ & $\begin{array}{l}\mathbf{E} \\
\mathbf{E} \\
\mathbf{E} \\
\mathbf{D} \\
\mathbf{E}\end{array}$ & $\begin{array}{l}\mathbf{E} \\
\mathbf{E} \\
\mathbf{E} \\
\mathbf{E} \\
\mathbf{E}\end{array}$ & $\begin{array}{l}\mathbf{E} \\
\mathbf{E} \\
\mathbf{E} \\
\mathbf{E} \\
\mathbf{E}\end{array}$ \\
\hline $\begin{array}{l}\mathbf{P} \\
\mathbf{P} \\
\mathbf{P} \\
\mathbf{P} \\
\mathbf{P}\end{array}$ & $\begin{array}{l}-165 \\
-164 \\
-164 \\
-162\end{array}$ & $\begin{array}{l}\text { VEM-460-47 } \\
\text { VEM-459-47 } \\
\text { VEM-458-47 } \\
\text { VEM-457-47 } \\
\text { VEM-456-47 }\end{array}$ & $\begin{array}{l}\mathbf{C} \\
\mathbf{C} \\
\mathbf{C} \\
\mathbf{C} \\
\mathbf{C}\end{array}$ & $\begin{array}{l}\mathbf{F} \\
\mathbf{F} \\
\mathbf{F} \\
\mathbf{F} \\
\mathbf{F}\end{array}$ & $\begin{array}{l}\text { A } \\
\text { C } \\
\text { C } \\
\text { C } \\
\text { C }\end{array}$ & $\begin{array}{l}\mathbf{E} \\
\mathbf{E} \\
\mathbf{E} \\
\mathbf{E} \\
\mathbf{E}\end{array}$ & $\begin{array}{l}\text { ND } \\
\text { ND } \\
\text { ND } \\
\text { ND } \\
\text { ND }\end{array}$ & $\begin{array}{l}\mathbf{G} \\
\mathbf{G} \\
\mathbf{G} \\
\mathbf{G} \\
\mathbf{G}\end{array}$ & $\begin{array}{l}\text { C } \\
\text { C } \\
\text { C } \\
\text { C } \\
\text { C }\end{array}$ & $\begin{array}{l}\text { C } \\
\text { D } \\
\text { C } \\
\text { D } \\
\text { D }\end{array}$ & $\begin{array}{l}\mathbf{E} \\
\mathbf{E} \\
\mathbf{E} \\
\mathbf{E} \\
\mathbf{E}\end{array}$ & $\begin{array}{l}\mathbf{F} \\
\mathbf{F} \\
\mathbf{F} \\
\mathbf{F} \\
\mathbf{F}\end{array}$ & $\begin{array}{l}\mathbf{E} \\
\mathbf{E} \\
\mathbf{E} \\
\mathbf{E} \\
\mathbf{E}\end{array}$ & $\begin{array}{l}\text { B } \\
\mathbf{A} \\
\mathbf{A} \\
\mathbf{A} \\
\mathbf{A}\end{array}$ & $\begin{array}{l}\mathbf{G} \\
\mathbf{G} \\
\mathbf{G} \\
\mathbf{G} \\
\mathbf{G}\end{array}$ & $\begin{array}{l}\mathbf{E} \\
\mathbf{E} \\
\mathbf{E} \\
\mathbf{D} \\
\mathbf{D}\end{array}$ & $\begin{array}{l}\mathbf{F} \\
\mathbf{F} \\
\mathbf{F} \\
\mathbf{F} \\
\mathbf{F}\end{array}$ & $\begin{array}{l}\mathbf{E} \\
\mathbf{E} \\
\mathbf{E} \\
\mathbf{E} \\
\mathbf{E}\end{array}$ & $\begin{array}{l}\mathbf{E} \\
\mathbf{E} \\
\mathbf{E} \\
\mathbf{E} \\
\mathbf{E}\end{array}$ & $\begin{array}{l}\mathbf{E} \\
\mathbf{E} \\
\mathbf{N D} \\
\text { ND } \\
\mathbf{N D}\end{array}$ & $\begin{array}{l}\mathbf{E} \\
\mathbf{E} \\
\mathbf{E} \\
\mathbf{E} \\
\mathbf{E}\end{array}$ \\
\hline $\begin{array}{l}\mathbf{P} \\
\mathbf{P} \\
\mathbf{P} \\
\mathbf{P} \\
\mathbf{P}\end{array}$ & $\begin{array}{l}-161 \\
-160 \\
-159 \\
-158 \\
-157\end{array}$ & $\begin{array}{l}\text { VEM-455-47 } \\
\text { VEM-454-47 } \\
\text { VEM-453-47 } \\
\text { VEM-452-47 } \\
\text { VEM-451-47 }\end{array}$ & $\begin{array}{l}\text { C } \\
\text { C } \\
\text { C } \\
\text { C } \\
\text { C }\end{array}$ & $\begin{array}{l}\mathbf{F} \\
\mathbf{F} \\
\mathbf{F} \\
\mathbf{F} \\
\mathbf{F}\end{array}$ & $\begin{array}{l}\text { A } \\
\text { C } \\
\text { C } \\
\text { A } \\
\text { C }\end{array}$ & $\begin{array}{l}\mathbf{E} \\
\mathbf{E} \\
\mathbf{E} \\
\mathbf{E} \\
\mathbf{E}\end{array}$ & $\begin{array}{l}\text { ND } \\
\text { ND } \\
\text { ND } \\
\text { ND } \\
\text { ND }\end{array}$ & $\begin{array}{l}\mathbf{G} \\
\mathbf{G} \\
\mathbf{G} \\
\mathbf{G} \\
\mathbf{G}\end{array}$ & $\begin{array}{l}\mathrm{C} \\
\mathrm{C} \\
\mathrm{C} \\
\mathrm{C} \\
\mathrm{C}\end{array}$ & $\begin{array}{l}\text { C } \\
\text { D } \\
\text { D } \\
\text { C } \\
\text { D }\end{array}$ & $\begin{array}{l}\mathbf{E} \\
\mathrm{E} \\
\mathrm{E} \\
\mathbf{E} \\
\mathbf{E}\end{array}$ & $\begin{array}{l}\mathbf{F} \\
\mathbf{F} \\
\mathbf{F} \\
\mathbf{F} \\
\mathbf{F}\end{array}$ & $\begin{array}{l}\mathbf{E} \\
\mathbf{E} \\
\mathbf{E} \\
\mathbf{E} \\
\mathbf{E}\end{array}$ & $\begin{array}{l}\text { C } \\
\text { A } \\
\text { A } \\
\text { A } \\
\text { A }\end{array}$ & $\begin{array}{l}\mathbf{G} \\
\mathbf{G} \\
\mathbf{G} \\
\mathbf{G} \\
\mathbf{G}\end{array}$ & $\begin{array}{l}\mathbf{E} \\
\mathbf{D} \\
\mathbf{D} \\
\mathbf{E} \\
\mathbf{D}\end{array}$ & $\begin{array}{l}\mathbf{F} \\
\mathbf{F} \\
\mathbf{F} \\
\mathbf{F} \\
\mathbf{F}\end{array}$ & $\begin{array}{l}\mathbf{E} \\
\mathbf{E} \\
\mathbf{E} \\
\mathbf{E} \\
\mathbf{E}\end{array}$ & $\begin{array}{l}\mathbf{E} \\
\mathbf{E} \\
\mathbf{E} \\
\mathbf{E} \\
\mathbf{E}\end{array}$ & $\begin{array}{l}\text { ND } \\
\text { ND } \\
\text { ND } \\
\text { ND } \\
\text { ND }\end{array}$ & $\begin{array}{l}\mathbf{E} \\
\mathbf{E} \\
\mathbf{E} \\
\mathbf{E} \\
\mathbf{E}\end{array}$ \\
\hline $\begin{array}{l}\mathbf{P} \\
\mathbf{P} \\
\mathbf{P} \\
\mathbf{P} \\
\mathbf{P}\end{array}$ & $\begin{array}{l}-156 \\
-155 \\
-154 \\
-153 \\
-152\end{array}$ & $\begin{array}{l}\text { VEM-450-47 } \\
\text { VEM-449-47 } \\
\text { VEM-448-47 } \\
\text { VEM-447-47 } \\
\text { VEM-446-47 }\end{array}$ & $\begin{array}{l}\text { C } \\
\text { C } \\
\text { D } \\
\text { C } \\
\text { C }\end{array}$ & $\begin{array}{l}\mathbf{F} \\
\mathbf{F} \\
\mathbf{F} \\
\mathbf{F} \\
\mathbf{F}\end{array}$ & $\begin{array}{l}\text { A } \\
\text { C } \\
\text { C } \\
\text { C } \\
\text { C }\end{array}$ & $\begin{array}{l}\mathbf{E} \\
\mathbf{E} \\
\mathbf{E} \\
\mathbf{E} \\
\mathbf{E}\end{array}$ & $\begin{array}{l}\text { ND } \\
\text { ND } \\
\text { ND } \\
\text { ND } \\
\text { ND }\end{array}$ & $\begin{array}{l}\mathbf{G} \\
\mathbf{G} \\
\mathbf{G} \\
\mathbf{G} \\
\mathbf{G}\end{array}$ & $\begin{array}{l}\mathrm{C} \\
\mathbf{C} \\
\mathbf{C} \\
\mathrm{C} \\
\mathrm{C}\end{array}$ & $\begin{array}{l}\mathrm{D} \\
\mathrm{D} \\
\mathrm{D} \\
\mathrm{D} \\
\mathrm{D}\end{array}$ & $\begin{array}{l}\mathbf{E} \\
\mathbf{E} \\
\mathbf{E} \\
\mathbf{E} \\
\mathbf{E}\end{array}$ & $\begin{array}{l}\mathbf{F} \\
\mathbf{F} \\
\mathbf{F} \\
\mathbf{F} \\
\mathbf{F}\end{array}$ & $\begin{array}{l}\mathbf{E} \\
\mathbf{E} \\
\mathbf{E} \\
\mathbf{E} \\
\mathbf{E}\end{array}$ & $\begin{array}{l}\text { A } \\
\text { A } \\
\text { C } \\
\text { A } \\
\text { A }\end{array}$ & $\begin{array}{c}\text { G } \\
\mathbf{G} \\
\text { ND } \\
\mathbf{G} \\
\mathbf{G}\end{array}$ & $\begin{array}{c}\text { E } \\
\text { D } \\
\text { ND } \\
\text { D } \\
\text { D }\end{array}$ & $\begin{array}{l}\mathbf{E} \\
\mathbf{F} \\
\mathbf{F} \\
\mathbf{F} \\
\mathbf{F}\end{array}$ & $\begin{array}{l}\mathbf{E} \\
\mathbf{E} \\
\mathbf{E} \\
\mathbf{E} \\
\mathbf{E}\end{array}$ & $\begin{array}{l}\mathbf{E} \\
\mathbf{E} \\
\mathbf{E} \\
\mathbf{E} \\
\mathbf{E}\end{array}$ & $\begin{array}{l}\mathbf{E} \\
\mathbf{E} \\
\mathbf{N} \\
\mathbf{E} \\
\mathbf{E}\end{array}$ & $\begin{array}{l}\mathbf{E} \\
\mathbf{E} \\
\mathbf{E} \\
\mathbf{E} \\
\mathbf{E}\end{array}$ \\
\hline $\begin{array}{l}\mathbf{P} \\
\mathbf{P} \\
\mathbf{P} \\
\mathbf{P}\end{array}$ & $\begin{array}{l}-151 \\
-150 \\
-149 \\
-148\end{array}$ & $\begin{array}{l}\text { VEM-445-47 } \\
\text { VEM-444-47 } \\
\text { VEM-443-47 } \\
\text { VEM-442-47 }\end{array}$ & $\begin{array}{l}\text { D } \\
\text { C } \\
\text { C } \\
\text { C }\end{array}$ & $\begin{array}{l}\mathbf{F} \\
\mathbf{F} \\
\mathbf{F} \\
\mathbf{F}\end{array}$ & $\begin{array}{l}\mathbf{A} \\
\mathbf{A} \\
\mathbf{C} \\
\mathbf{A}\end{array}$ & $\begin{array}{l}\mathbf{E} \\
\mathbf{E} \\
\mathbf{E} \\
\mathbf{E}\end{array}$ & $\begin{array}{l}\text { ND } \\
\text { ND } \\
\text { ND } \\
\text { ND }\end{array}$ & $\begin{array}{l}\mathbf{G} \\
\mathbf{G} \\
\mathbf{G} \\
\mathbf{G}\end{array}$ & $\begin{array}{l}\mathrm{C} \\
\mathrm{C} \\
\mathrm{C} \\
\mathrm{C}\end{array}$ & $\begin{array}{l}\text { D } \\
\text { D } \\
\text { D } \\
\text { D }\end{array}$ & $\begin{array}{l}\mathbf{F} \\
\mathbf{F} \\
\mathbf{F} \\
\mathbf{E}\end{array}$ & $\begin{array}{l}\mathbf{F} \\
\mathbf{F} \\
\mathbf{F} \\
\mathbf{F}\end{array}$ & $\begin{array}{l}\mathbf{E} \\
\mathbf{E} \\
\mathbf{E} \\
\mathbf{E}\end{array}$ & $\begin{array}{l}\text { C } \\
\text { C } \\
\text { A } \\
\text { B }\end{array}$ & $\begin{array}{c}\mathbf{G} \\
\mathbf{G} \\
\mathbf{G} \\
\mathbf{G}\end{array}$ & $\begin{array}{l}\mathbf{E} \\
\mathbf{E} \\
\mathbf{E} \\
\mathbf{E}\end{array}$ & $\begin{array}{l}\mathbf{E} \\
\mathbf{E} \\
\mathbf{F} \\
\mathbf{E}\end{array}$ & $\begin{array}{l}\mathbf{E} \\
\mathbf{E} \\
\mathbf{E} \\
\mathbf{E}\end{array}$ & $\begin{array}{l}\mathbf{E} \\
\mathbf{E} \\
\mathbf{E} \\
\mathbf{D}\end{array}$ & $\begin{array}{l}\mathbf{E} \\
\mathbf{E} \\
\mathbf{E} \\
\mathbf{E}\end{array}$ & $\begin{array}{l}\mathbf{E} \\
\mathbf{E} \\
\mathbf{E} \\
\mathbf{E}\end{array}$ \\
\hline
\end{tabular}




\begin{tabular}{|c|c|c|c|c|c|c|c|c|c|c|c|c|c|c|c|c|c|c|c|c|c|c|}
\hline $\begin{array}{l}\mathbf{P} \\
\mathbf{P} \\
\mathbf{P} \\
\mathbf{P} \\
\mathbf{P}\end{array}$ & $\begin{array}{l}-147 \\
-146 \\
-145 \\
-144 \\
-143\end{array}$ & $\begin{array}{l}\text { VEM-441-47 } \\
\text { VEM-440-47 } \\
\text { VEM-439-47 } \\
\text { VEM-438-47 } \\
\text { VEM-437-47 }\end{array}$ & $\begin{array}{l}\mathbf{C} \\
\mathbf{C} \\
\mathbf{C} \\
\mathbf{C} \\
\mathbf{C}\end{array}$ & $\begin{array}{l}\mathbf{F} \\
\mathbf{F} \\
\mathbf{F} \\
\mathbf{F} \\
\mathbf{F}\end{array}$ & $\begin{array}{l}\text { A } \\
\text { A } \\
\text { A } \\
\text { A } \\
\text { A }\end{array}$ & $\begin{array}{l}\mathbf{E} \\
\mathbf{E} \\
\mathbf{E} \\
\mathbf{E} \\
\mathbf{E}\end{array}$ & $\begin{array}{l}\text { ND } \\
\text { ND } \\
\text { ND } \\
\text { ND } \\
\text { ND }\end{array}$ & $\begin{array}{l}\mathbf{G} \\
\mathbf{G} \\
\mathbf{G} \\
\mathbf{G} \\
\mathbf{G}\end{array}$ & $\begin{array}{l}\mathbf{C} \\
\mathbf{C} \\
\mathbf{C} \\
\mathbf{C} \\
\mathbf{C}\end{array}$ & $\begin{array}{l}\text { D } \\
\text { D } \\
\text { D } \\
\text { D } \\
\text { D }\end{array}$ & $\begin{array}{l}\mathbf{E} \\
\mathbf{E} \\
\mathbf{E} \\
\mathbf{E} \\
\mathbf{E}\end{array}$ & $\begin{array}{l}\mathbf{F} \\
\mathbf{F} \\
\mathbf{F} \\
\mathbf{F} \\
\mathbf{F}\end{array}$ & $\begin{array}{l}\mathbf{E} \\
\mathbf{E} \\
\mathbf{E} \\
\mathbf{E} \\
\mathbf{E}\end{array}$ & $\begin{array}{l}\text { B } \\
\mathbf{C} \\
\mathbf{A} \\
\mathbf{C} \\
\mathbf{A}\end{array}$ & $\begin{array}{l}\mathbf{G} \\
\mathbf{G} \\
\mathbf{G} \\
\mathbf{G} \\
\mathbf{G}\end{array}$ & $\begin{array}{l}\mathbf{E} \\
\mathbf{E} \\
\mathbf{D} \\
\mathbf{E} \\
\mathbf{D}\end{array}$ & $\begin{array}{l}\mathbf{E} \\
\mathbf{E} \\
\mathbf{E} \\
\mathbf{E} \\
\mathbf{E}\end{array}$ & $\begin{array}{l}\mathbf{E} \\
\mathbf{E} \\
\mathbf{E} \\
\mathbf{E} \\
\mathbf{E}\end{array}$ & $\begin{array}{l}\text { D } \\
\text { D } \\
\text { D } \\
\text { D } \\
\text { D }\end{array}$ & $\begin{array}{l}\mathbf{E} \\
\mathbf{E} \\
\mathbf{E} \\
\mathbf{E} \\
\mathbf{E}\end{array}$ & $\begin{array}{l}\mathbf{E} \\
\mathbf{E} \\
\mathbf{E} \\
\mathbf{E} \\
\mathbf{E}\end{array}$ & \\
\hline $\begin{array}{l}\mathbf{P} \\
\mathbf{P} \\
\mathbf{P} \\
\mathbf{P} \\
\mathbf{P}\end{array}$ & $\begin{array}{l}-142 \\
-141 \\
-140 \\
-139 \\
-138\end{array}$ & $\begin{array}{l}\text { VEM-436-47 } \\
\text { VEM-435-47 } \\
\text { VEM-434-47 } \\
\text { VEM-433-47 } \\
\text { VEM-432-47 }\end{array}$ & $\begin{array}{l}\text { C } \\
\mathbf{C} \\
\mathbf{C} \\
\mathbf{C} \\
\mathbf{C}\end{array}$ & $\begin{array}{l}\mathbf{F} \\
\mathbf{F} \\
\mathbf{F} \\
\mathbf{F} \\
\mathbf{F}\end{array}$ & $\begin{array}{l}\text { A } \\
\text { A } \\
\text { B } \\
\text { A } \\
\text { A }\end{array}$ & $\begin{array}{l}\mathbf{E} \\
\mathbf{E} \\
\mathbf{E} \\
\mathbf{E} \\
\mathbf{E}\end{array}$ & $\begin{array}{l}\text { ND } \\
\text { ND } \\
\text { ND } \\
\text { ND } \\
\text { ND }\end{array}$ & $\begin{array}{l}\mathbf{G} \\
\mathbf{G} \\
\mathbf{G} \\
\mathbf{G} \\
\mathbf{G}\end{array}$ & $\begin{array}{l}\text { C } \\
\mathbf{C} \\
\mathbf{C} \\
\mathbf{C} \\
\mathbf{C}\end{array}$ & $\begin{array}{l}\text { D } \\
\text { D } \\
\text { D } \\
\text { D } \\
\text { D }\end{array}$ & $\begin{array}{l}\mathbf{E} \\
\mathbf{E} \\
\mathbf{E} \\
\mathbf{F} \\
\mathbf{F}\end{array}$ & $\begin{array}{l}\mathbf{F} \\
\mathbf{F} \\
\mathbf{F} \\
\mathbf{F} \\
\mathbf{F}\end{array}$ & $\begin{array}{l}\mathbf{E} \\
\mathbf{E} \\
\mathbf{E} \\
\mathbf{E} \\
\mathbf{E}\end{array}$ & $\begin{array}{l}\text { A } \\
\mathbf{A} \\
\mathbf{A} \\
\mathbf{C} \\
\mathbf{C}\end{array}$ & $\begin{array}{l}\mathbf{G} \\
\mathbf{G} \\
\mathbf{G} \\
\mathbf{G} \\
\mathbf{G}\end{array}$ & $\begin{array}{l}\mathbf{D} \\
\mathbf{E} \\
\mathbf{E} \\
\mathbf{E} \\
\mathbf{E}\end{array}$ & $\begin{array}{l}\mathbf{E} \\
\mathbf{E} \\
\mathbf{E} \\
\mathbf{E} \\
\mathbf{E}\end{array}$ & $\begin{array}{l}\mathbf{E} \\
\mathbf{E} \\
\mathbf{E} \\
\mathbf{E} \\
\mathbf{E}\end{array}$ & $\begin{array}{l}\mathbf{D} \\
\mathbf{D} \\
\mathbf{E} \\
\mathbf{D} \\
\mathbf{D}\end{array}$ & $\begin{array}{l}\mathbf{E} \\
\mathbf{E} \\
\mathbf{E} \\
\mathbf{E} \\
\mathbf{E}\end{array}$ & $\begin{array}{l}\mathbf{E} \\
\mathbf{E} \\
\mathbf{E} \\
\mathbf{E} \\
\mathbf{E}\end{array}$ & \\
\hline $\begin{array}{l}\mathbf{P} \\
\mathbf{P} \\
\mathbf{P} \\
\mathbf{P}\end{array}$ & $\begin{array}{l}-137 \\
-- \\
-136 \\
-135 \\
-134\end{array}$ & $\begin{array}{l}\text { VEM-431-47 } \\
\text { VEM-430-47 } \\
\text { VEM-429-47 } \\
\text { VEM-428-47 } \\
\text { VEM-427-47 }\end{array}$ & $\begin{array}{l}\mathbf{C} \\
\mathbf{C} \\
\mathbf{C} \\
\mathbf{C} \\
\mathbf{C}\end{array}$ & $\begin{array}{l}\mathbf{F} \\
\mathbf{F} \\
\mathbf{F} \\
\mathbf{F} \\
\mathbf{F}\end{array}$ & $\begin{array}{l}\mathbf{A} \\
\mathbf{A} \\
\mathbf{A} \\
\mathbf{A} \\
\mathbf{A}\end{array}$ & $\begin{array}{l}\mathbf{E} \\
\mathbf{E} \\
\mathbf{E} \\
\mathbf{E} \\
\mathbf{E}\end{array}$ & $\begin{array}{l}\text { ND } \\
\text { ND } \\
\text { ND } \\
\text { ND } \\
\text { ND }\end{array}$ & $\begin{array}{l}\mathbf{G} \\
\mathbf{G} \\
\mathbf{G} \\
\mathbf{G} \\
\mathbf{G}\end{array}$ & $\begin{array}{l}\text { C } \\
\text { D } \\
\text { C } \\
\text { C } \\
\text { C }\end{array}$ & $\begin{array}{l}\mathbf{D} \\
\mathbf{C} \\
\mathbf{D} \\
\mathbf{D} \\
\mathbf{D}\end{array}$ & $\begin{array}{l}\mathbf{F} \\
\mathbf{F} \\
\mathbf{E} \\
\mathbf{E} \\
\mathbf{E}\end{array}$ & $\begin{array}{l}\mathbf{F} \\
\mathbf{F} \\
\mathbf{F} \\
\mathbf{F} \\
\mathbf{F}\end{array}$ & $\begin{array}{l}\mathbf{E} \\
\mathbf{E} \\
\mathbf{E} \\
\mathbf{E} \\
\mathbf{E}\end{array}$ & $\begin{array}{l}\text { A } \\
\text { C } \\
\text { A } \\
\text { A } \\
\text { A }\end{array}$ & $\begin{array}{l}\mathbf{F} \\
\mathbf{G} \\
\mathbf{F} \\
\mathbf{F} \\
\mathbf{F}\end{array}$ & $\begin{array}{l}\mathbf{E} \\
\mathbf{E} \\
\mathbf{E} \\
\mathbf{E} \\
\mathbf{E}\end{array}$ & $\begin{array}{l}\mathbf{E} \\
\mathbf{E} \\
\mathbf{D} \\
\mathbf{D} \\
\mathbf{D}\end{array}$ & $\begin{array}{l}\mathbf{E} \\
\mathbf{E} \\
\mathbf{E} \\
\mathbf{E} \\
\mathbf{E}\end{array}$ & $\begin{array}{l}\text { D } \\
\text { D } \\
\text { D } \\
\text { D } \\
\text { D }\end{array}$ & $\begin{array}{l}\mathbf{E} \\
\mathbf{E} \\
\mathbf{E} \\
\mathbf{E} \\
\mathbf{E}\end{array}$ & $\begin{array}{l}\mathbf{E} \\
\mathbf{E} \\
\mathbf{E} \\
\mathbf{E} \\
\mathbf{E}\end{array}$ & \\
\hline $\begin{array}{l}\mathbf{P} \\
\mathbf{P} \\
\mathbf{P} \\
\mathbf{P} \\
\mathbf{P}\end{array}$ & $\begin{array}{l}-133 \\
-132 \\
-131 \\
-130 \\
-129\end{array}$ & $\begin{array}{l}\text { VEM-426-47 } \\
\text { VEM-425-47 } \\
\text { VEM-424-47 } \\
\text { VEM-423-47 } \\
\text { VEM-422-47 }\end{array}$ & $\begin{array}{l}\text { C } \\
\text { C } \\
\text { C } \\
\text { C } \\
\text { C }\end{array}$ & $\begin{array}{l}\mathbf{F} \\
\mathbf{F} \\
\mathbf{F} \\
\mathbf{F} \\
\mathbf{F}\end{array}$ & $\begin{array}{l}\text { A } \\
\text { A } \\
\text { A } \\
\text { A } \\
\text { A }\end{array}$ & $\begin{array}{l}\mathbf{E} \\
\mathbf{E} \\
\mathbf{E} \\
\mathbf{E} \\
\mathbf{E}\end{array}$ & $\begin{array}{l}\text { ND } \\
\text { ND } \\
\text { ND } \\
\text { ND } \\
\text { ND }\end{array}$ & $\begin{array}{l}\mathbf{G} \\
\mathbf{G} \\
\mathbf{G} \\
\mathbf{G} \\
\mathbf{G}\end{array}$ & $\begin{array}{l}\mathbf{C} \\
\mathbf{C} \\
\mathbf{C} \\
\mathbf{C} \\
\mathbf{C}\end{array}$ & $\begin{array}{l}\mathbf{D} \\
\mathbf{D} \\
\mathbf{D} \\
\mathbf{D} \\
\mathbf{D}\end{array}$ & $\begin{array}{l}\mathbf{E} \\
\mathbf{E} \\
\mathbf{E} \\
\mathbf{E} \\
\mathbf{E}\end{array}$ & $\begin{array}{l}\mathbf{F} \\
\mathbf{F} \\
\mathbf{F} \\
\mathbf{F} \\
\mathbf{F}\end{array}$ & $\begin{array}{l}\mathbf{E} \\
\mathbf{E} \\
\mathbf{E} \\
\mathbf{E} \\
\mathbf{E}\end{array}$ & $\begin{array}{l}\text { A } \\
\text { A } \\
\text { A } \\
\text { A } \\
\text { A }\end{array}$ & $\begin{array}{l}\mathbf{F} \\
\mathbf{F} \\
\mathbf{F} \\
\mathbf{F} \\
\mathbf{F}\end{array}$ & $\begin{array}{l}\mathbf{E} \\
\mathbf{D} \\
\mathbf{E} \\
\mathbf{E} \\
\mathbf{E}\end{array}$ & $\begin{array}{l}D \\
D \\
D \\
D \\
D\end{array}$ & $\begin{array}{l}\mathbf{E} \\
\mathbf{E} \\
\mathbf{E} \\
\mathbf{E} \\
\mathbf{E}\end{array}$ & $\begin{array}{l}\mathbf{D} \\
\mathbf{D} \\
\mathbf{D} \\
\mathbf{D} \\
\mathbf{D}\end{array}$ & $\begin{array}{l}\mathbf{E} \\
\mathbf{E} \\
\mathbf{E} \\
\mathbf{E} \\
\mathbf{E}\end{array}$ & $\begin{array}{l}\mathbf{E} \\
\mathbf{E} \\
\mathbf{E} \\
\mathbf{E} \\
\mathbf{E}\end{array}$ & ? \\
\hline $\begin{array}{l}\mathbf{P} \\
\mathbf{P} \\
\mathbf{P} \\
\mathbf{P} \\
\mathbf{P}\end{array}$ & $\begin{array}{l}-128 \\
-127 \\
-126 \\
-125 \\
-124\end{array}$ & $\begin{array}{l}\text { VEM-421-47 } \\
\text { VEM-420-47 } \\
\text { VEM-419-47 } \\
\text { VEM-418-47 } \\
\text { VEM-417-47 }\end{array}$ & $\begin{array}{l}\mathbf{C} \\
\mathbf{C} \\
\mathbf{C} \\
\mathbf{C} \\
\mathbf{C}\end{array}$ & $\begin{array}{l}\mathbf{F} \\
\mathbf{F} \\
\mathbf{F} \\
\mathbf{F} \\
\mathbf{F}\end{array}$ & $\begin{array}{l}\text { A } \\
\text { A } \\
\text { A } \\
\text { A } \\
\text { A }\end{array}$ & $\begin{array}{l}\mathbf{E} \\
\mathbf{E} \\
\mathbf{D} \\
\mathbf{D} \\
\mathbf{D}\end{array}$ & $\begin{array}{l}\text { ND } \\
\text { ND } \\
\text { ND } \\
\text { ND } \\
\text { ND }\end{array}$ & $\begin{array}{l}\mathbf{G} \\
\mathbf{G} \\
\mathbf{G} \\
\mathbf{G} \\
\mathbf{G}\end{array}$ & $\begin{array}{l}\text { C } \\
\mathbf{C} \\
\mathbf{C} \\
\mathbf{C} \\
\mathrm{C}\end{array}$ & $\begin{array}{l}\text { D } \\
\text { D } \\
\text { D } \\
\text { D } \\
\text { D }\end{array}$ & $\begin{array}{l}\mathbf{E} \\
\mathbf{E} \\
\mathbf{E} \\
\mathbf{E} \\
\mathbf{E}\end{array}$ & $\begin{array}{l}\mathbf{F} \\
\mathbf{F} \\
\mathbf{F} \\
\mathbf{F} \\
\mathbf{F}\end{array}$ & $\begin{array}{l}\mathbf{E} \\
\mathbf{F} \\
\mathbf{E} \\
\mathbf{E} \\
\mathbf{E}\end{array}$ & $\begin{array}{l}\text { A } \\
\text { A } \\
\text { A } \\
\text { A } \\
\text { A }\end{array}$ & $\begin{array}{l}\mathbf{F} \\
\mathbf{F} \\
\mathbf{F} \\
\mathbf{F} \\
\mathbf{F}\end{array}$ & $\begin{array}{l}\text { D } \\
\text { D } \\
\text { D } \\
\text { D } \\
\text { D }\end{array}$ & $\begin{array}{l}\mathbf{E} \\
\mathbf{E} \\
\mathbf{E} \\
\mathbf{E} \\
\mathbf{D}\end{array}$ & $\begin{array}{l}\mathbf{E} \\
\mathbf{E} \\
\mathbf{E} \\
\mathbf{E} \\
\mathbf{E}\end{array}$ & $\begin{array}{l}\text { D } \\
\text { D } \\
\text { D } \\
\text { D } \\
\text { D }\end{array}$ & $\begin{array}{l}\mathbf{E} \\
\mathbf{E} \\
\mathbf{E} \\
\mathbf{E} \\
\mathbf{E}\end{array}$ & $\begin{array}{l}\mathbf{E} \\
\mathbf{E} \\
\mathbf{E} \\
\mathbf{E} \\
\mathbf{E}\end{array}$ & \\
\hline $\begin{array}{l}\mathbf{P} \\
\mathbf{P} \\
\mathbf{P} \\
\mathbf{P} \\
\mathbf{P}\end{array}$ & $\begin{array}{l}-123 \\
-122 \\
-121 \\
-120 \\
-119\end{array}$ & $\begin{array}{l}\text { VEM-416-47 } \\
\text { VEM-415-47 } \\
\text { VEM-414-47 } \\
\text { VEM-413-47 } \\
\text { VEM-412-47 }\end{array}$ & $\begin{array}{l}\mathbf{C} \\
\mathbf{C} \\
\mathbf{C} \\
\mathbf{C} \\
\mathbf{C}\end{array}$ & $\begin{array}{l}\mathbf{F} \\
\mathbf{F} \\
\mathbf{F} \\
\mathbf{F} \\
\mathbf{F}\end{array}$ & $\begin{array}{l}\text { A } \\
\text { A } \\
\text { A } \\
\text { A } \\
\text { A }\end{array}$ & $\begin{array}{l}\mathbf{E} \\
\mathbf{E} \\
\mathbf{E} \\
\mathbf{D} \\
\mathbf{E}\end{array}$ & $\begin{array}{l}\text { ND } \\
\text { ND } \\
\text { ND } \\
\text { ND } \\
\text { ND }\end{array}$ & $\begin{array}{l}\mathbf{G} \\
\mathbf{G} \\
\mathbf{G} \\
\mathbf{G} \\
\mathbf{G}\end{array}$ & $\begin{array}{l}\text { C } \\
\text { C } \\
\text { C } \\
\text { C } \\
\text { C }\end{array}$ & $\begin{array}{l}\text { D } \\
\text { C } \\
\text { D } \\
\text { D } \\
\text { D }\end{array}$ & $\begin{array}{l}\mathbf{E} \\
\mathbf{E} \\
\mathbf{E} \\
\mathbf{E} \\
\mathbf{E}\end{array}$ & $\begin{array}{l}\mathbf{F} \\
\mathbf{F} \\
\mathbf{F} \\
\mathbf{F} \\
\mathbf{F}\end{array}$ & $\begin{array}{l}\mathbf{E} \\
\mathbf{E} \\
\mathbf{E} \\
\mathbf{E} \\
\mathbf{E}\end{array}$ & $\begin{array}{l}\text { A } \\
\text { A } \\
\text { A } \\
\text { A } \\
\text { A }\end{array}$ & $\begin{array}{l}\mathbf{G} \\
\mathbf{G} \\
\mathbf{G} \\
\mathbf{G} \\
\mathbf{G}\end{array}$ & $\begin{array}{l}\mathbf{D} \\
\mathbf{D} \\
\mathbf{D} \\
\mathbf{D} \\
\mathbf{D}\end{array}$ & $\begin{array}{l}\mathbf{E} \\
\mathbf{E} \\
\mathbf{D} \\
\mathbf{E} \\
\mathbf{D}\end{array}$ & $\begin{array}{l}\mathbf{E} \\
\mathbf{E} \\
\mathbf{E} \\
\mathbf{E} \\
\mathbf{E}\end{array}$ & $\begin{array}{l}\mathbf{D} \\
\mathbf{D} \\
\mathbf{D} \\
\mathbf{D} \\
\mathbf{D}\end{array}$ & $\begin{array}{l}\mathbf{E} \\
\mathbf{E} \\
\mathbf{E} \\
\mathbf{E} \\
\mathbf{E}\end{array}$ & $\begin{array}{l}\mathbf{E} \\
\mathbf{E} \\
\mathbf{E} \\
\mathbf{E} \\
\mathbf{E}\end{array}$ & $A$ \\
\hline $\begin{array}{l}\mathbf{P} \\
\mathbf{P} \\
\mathbf{P} \\
\mathbf{P} \\
\mathbf{P}\end{array}$ & $\begin{array}{l}-118 \\
-117 \\
-116 \\
-115 \\
-114\end{array}$ & $\begin{array}{l}\text { VEM-411-47 } \\
\text { VEM-410-47 } \\
\text { VEM-409-47 } \\
\text { VEM-408-47 } \\
\text { VEM-407-47 }\end{array}$ & $\begin{array}{l}\mathbf{C} \\
\mathbf{C} \\
\mathbf{C} \\
\mathbf{C} \\
\mathbf{C}\end{array}$ & $\begin{array}{l}\mathbf{F} \\
\mathbf{F} \\
\mathbf{F} \\
\mathbf{F} \\
\mathbf{F}\end{array}$ & $\begin{array}{l}\text { A } \\
\text { B } \\
\text { A } \\
\text { A } \\
\text { A }\end{array}$ & $\begin{array}{l}\mathbf{E} \\
\mathbf{E} \\
\mathbf{E} \\
\mathbf{E} \\
\mathbf{E}\end{array}$ & $\begin{array}{l}\text { ND } \\
\text { ND } \\
\text { ND } \\
\text { ND } \\
\text { ND }\end{array}$ & $\begin{array}{l}\mathbf{G} \\
\mathbf{G} \\
\mathbf{G} \\
\mathbf{G} \\
\mathbf{G}\end{array}$ & $\begin{array}{l}\mathbf{C} \\
\mathbf{C} \\
\mathbf{C} \\
\mathbf{C} \\
\mathbf{C}\end{array}$ & $\begin{array}{l}\text { D } \\
\text { D } \\
\text { D } \\
\text { D } \\
\text { D }\end{array}$ & $\begin{array}{l}\mathbf{E} \\
\mathbf{E} \\
\mathbf{E} \\
\mathbf{E} \\
\mathbf{E}\end{array}$ & $\begin{array}{l}\text { F } \\
\text { F } \\
\text { F } \\
\text { F }\end{array}$ & $\begin{array}{l}\mathbf{E} \\
\mathbf{E} \\
\mathbf{E} \\
\mathbf{E} \\
\mathbf{E}\end{array}$ & $\begin{array}{l}\text { A } \\
\text { A } \\
\text { A } \\
\text { A } \\
\text { A }\end{array}$ & $\begin{array}{l}\mathbf{G} \\
\mathbf{G} \\
\mathbf{G} \\
\mathbf{G} \\
\mathbf{G}\end{array}$ & $\begin{array}{l}\text { D } \\
\text { D } \\
\text { D } \\
\text { D } \\
\text { D }\end{array}$ & $\begin{array}{l}\mathbf{D} \\
\mathbf{E} \\
\mathbf{E} \\
\mathbf{E} \\
\mathbf{E}\end{array}$ & $\begin{array}{l}\mathbf{E} \\
\mathbf{E} \\
\mathbf{E} \\
\mathbf{E} \\
\mathbf{E}\end{array}$ & $\begin{array}{l}\mathbf{D} \\
\mathbf{D} \\
\mathbf{D} \\
\mathbf{D} \\
\mathbf{D}\end{array}$ & $\begin{array}{l}\mathbf{E} \\
\mathbf{E} \\
\mathbf{E} \\
\mathbf{E} \\
\mathbf{E}\end{array}$ & $\begin{array}{l}\mathbf{E} \\
\mathbf{E} \\
\mathbf{E} \\
\mathbf{E} \\
\mathbf{E}\end{array}$ & if \\
\hline $\begin{array}{l}\mathbf{P} \\
\mathbf{P} \\
\mathbf{P} \\
\mathbf{P} \\
\mathbf{P}\end{array}$ & $\begin{array}{l}-113 \\
-112 \\
-111 \\
-110 \\
-109\end{array}$ & $\begin{array}{l}\text { VEM-406-47 } \\
\text { VEM-405-47 } \\
\text { VEM-404-47 } \\
\text { VEM-403-47 } \\
\text { VEM-402-47 }\end{array}$ & $\begin{array}{l}\mathbf{C} \\
\mathbf{C} \\
\mathbf{C} \\
\mathbf{C} \\
\mathbf{C}\end{array}$ & $\begin{array}{l}\mathbf{F} \\
\mathbf{F} \\
\mathbf{F} \\
\mathbf{F} \\
\mathbf{F}\end{array}$ & $\begin{array}{l}\mathbf{A} \\
\mathbf{A} \\
\mathbf{C} \\
\mathbf{A} \\
\mathbf{A}\end{array}$ & $\begin{array}{l}\mathbf{E} \\
\mathbf{E} \\
\mathbf{E} \\
\mathbf{E} \\
\mathbf{E}\end{array}$ & $\begin{array}{l}\text { ND } \\
\text { ND } \\
\text { ND } \\
\text { ND } \\
\text { ND }\end{array}$ & $\begin{array}{l}\mathbf{G} \\
\mathbf{G} \\
\mathbf{G} \\
\mathbf{G} \\
\mathbf{G}\end{array}$ & $\begin{array}{l}\mathrm{C} \\
\mathrm{C} \\
\mathrm{C} \\
\mathrm{C} \\
\mathrm{C}\end{array}$ & $\begin{array}{l}D \\
D \\
D \\
D \\
D\end{array}$ & $\begin{array}{l}\mathbf{E} \\
\mathbf{E} \\
\mathrm{E} \\
\mathrm{E} \\
\mathrm{E}\end{array}$ & $\begin{array}{l}F \\
F \\
F \\
F \\
F\end{array}$ & $\begin{array}{l}\mathbf{E} \\
\mathbf{E} \\
\mathbf{E} \\
\mathbf{E} \\
\mathbf{E}\end{array}$ & $\begin{array}{l}\text { A } \\
\text { A } \\
\text { A } \\
\text { A } \\
\text { A }\end{array}$ & $\begin{array}{l}\mathbf{G} \\
\mathbf{G} \\
\mathbf{G} \\
\mathbf{G} \\
\mathbf{G}\end{array}$ & $\begin{array}{l}\text { D } \\
\text { D } \\
\text { D } \\
\text { D } \\
\text { D }\end{array}$ & $\begin{array}{l}\mathbf{E} \\
\mathbf{D} \\
\mathbf{E} \\
\mathbf{E} \\
\mathbf{E}\end{array}$ & $\begin{array}{l}\mathbf{E} \\
\mathbf{E} \\
\mathbf{E} \\
\mathbf{E} \\
\mathbf{E}\end{array}$ & $\begin{array}{l}\mathbf{E} \\
\mathbf{E} \\
\mathbf{E} \\
\mathbf{E} \\
\mathbf{E}\end{array}$ & $\begin{array}{l}\mathbf{E} \\
\mathbf{E} \\
\mathbf{E} \\
\mathbf{E} \\
\mathbf{E}\end{array}$ & $\begin{array}{l}\mathbf{E} \\
\mathbf{E} \\
\mathbf{E} \\
\mathbf{E} \\
\mathbf{E}\end{array}$ & (Ai) \\
\hline $\begin{array}{l}\mathbf{P} \\
\mathbf{P} \\
\mathbf{P} \\
\mathbf{P} \\
\mathbf{P}\end{array}$ & $\begin{array}{l}-108 \\
-107 \\
-106 \\
-105 \\
-104\end{array}$ & $\begin{array}{l}\text { VEM-401-47 } \\
\text { VEM-400-47 } \\
\text { VEM-399-47 } \\
\text { VEM-398-47 } \\
\text { VEM-397-47 }\end{array}$ & $\begin{array}{l}\mathbf{C} \\
\mathbf{C} \\
\mathbf{C} \\
\mathbf{C} \\
\mathbf{C}\end{array}$ & $\begin{array}{l}\mathbf{F} \\
\mathbf{F} \\
\mathbf{F} \\
\mathbf{F} \\
\mathbf{F}\end{array}$ & $\begin{array}{l}\text { A } \\
\mathbf{C} \\
\mathbf{A} \\
\mathbf{C} \\
\mathbf{A}\end{array}$ & $\begin{array}{l}\mathbf{E} \\
\mathbf{E} \\
\mathbf{E} \\
\mathbf{E} \\
\mathbf{D}\end{array}$ & $\begin{array}{l}\text { ND } \\
\text { ND } \\
\text { ND } \\
\text { ND } \\
\text { ND }\end{array}$ & $\begin{array}{l}\mathbf{G} \\
\mathbf{G} \\
\mathbf{G} \\
\mathbf{G} \\
\mathbf{G}\end{array}$ & $\begin{array}{l}\text { C } \\
\text { C } \\
\text { C } \\
\text { C } \\
\text { C }\end{array}$ & $\begin{array}{l}\text { D } \\
\text { D } \\
\text { B } \\
\text { D } \\
\text { D }\end{array}$ & $\begin{array}{l}\mathbf{E} \\
\mathbf{E} \\
\mathbf{E} \\
\mathbf{E} \\
\mathbf{E}\end{array}$ & $\begin{array}{l}F \\
F \\
F \\
F \\
F\end{array}$ & $\begin{array}{l}\mathbf{E} \\
\mathbf{E} \\
\mathbf{E} \\
\mathbf{E} \\
\mathbf{E}\end{array}$ & $\begin{array}{l}\text { A } \\
\text { A } \\
\text { B } \\
\text { A } \\
\text { A }\end{array}$ & $\begin{array}{l}\mathbf{G} \\
\mathbf{G} \\
\mathbf{G} \\
\mathbf{G} \\
\mathbf{F}\end{array}$ & $\begin{array}{l}\text { D } \\
\text { D } \\
\text { D } \\
\text { D } \\
\text { D }\end{array}$ & $\begin{array}{l}\mathbf{E} \\
\mathbf{E} \\
\mathbf{E} \\
\mathbf{E} \\
\mathbf{E}\end{array}$ & $\begin{array}{l}\mathbf{E} \\
\mathbf{E} \\
\mathbf{E} \\
\mathbf{E} \\
\mathbf{E}\end{array}$ & $\begin{array}{l}\mathbf{E} \\
\mathbf{E} \\
\mathbf{E} \\
\mathbf{E} \\
\mathbf{D}\end{array}$ & $\begin{array}{l}\mathbf{E} \\
\mathbf{N D} \\
\mathbf{E} \\
\mathbf{E} \\
\mathbf{E}\end{array}$ & $\begin{array}{l}\mathbf{E} \\
\mathbf{E} \\
\mathbf{E} \\
\mathbf{E} \\
\mathbf{E}\end{array}$ & \\
\hline
\end{tabular}




\begin{tabular}{|c|c|c|c|c|c|c|c|c|c|c|c|c|c|c|c|c|c|c|c|c|c|}
\hline $\begin{array}{c}\text { Bed } \\
\text { no. }\end{array}$ & Sample no. & Al & B & $\mathrm{Ca}$ & $\mathrm{Cr}$ & Co & $\mathrm{Cu}$ & $\mathrm{Fe}$ & Mg & $\mathbf{M n}$ & Mo & $\mathrm{Ni}$ & $\mathbf{S i}$ & Ag & $\mathrm{Na}$ & $\mathrm{Sr}$ & Ti & v & $\mathbf{Z n}$ & $\mathbf{Z r}$ & \\
\hline $\begin{array}{ll}P & -103 \\
P & -102 \\
P & -101 \\
P & -100 \\
P & -99\end{array}$ & $\begin{array}{l}\text { VEM-396-47 } \\
\text { VEM-395-47 } \\
\text { VEM-394-47 } \\
\text { VEM-393-47 } \\
\text { VEM-392-47 }\end{array}$ & $\begin{array}{l}\mathbf{C} \\
\mathbf{C} \\
\mathbf{C} \\
\mathbf{C} \\
\mathbf{C}\end{array}$ & $\begin{array}{l}\mathbf{F} \\
\mathbf{F} \\
\mathbf{F} \\
\mathbf{F}\end{array}$ & $\begin{array}{l}\text { B } \\
\text { C } \\
\text { C } \\
\text { B } \\
\text { A }\end{array}$ & $\begin{array}{l}\mathbf{D} \\
\mathbf{E} \\
\mathbf{E} \\
\mathbf{E} \\
\mathbf{E}\end{array}$ & $\begin{array}{l}\text { ND } \\
\text { ND } \\
\text { E } \\
\text { ND } \\
\text { ND }\end{array}$ & $\begin{array}{l}\mathbf{G} \\
\mathbf{G} \\
\mathbf{G} \\
\mathbf{G} \\
\mathbf{G}\end{array}$ & $\begin{array}{l}C \\
C \\
C \\
C \\
C\end{array}$ & $\begin{array}{l}\text { D } \\
\text { D } \\
\text { D } \\
\text { D } \\
\text { D }\end{array}$ & $\begin{array}{l}\mathbf{E} \\
\mathbf{E} \\
\mathbf{E} \\
\mathbf{E} \\
\mathbf{E}\end{array}$ & $\begin{array}{l}\mathbf{F} \\
\mathbf{F} \\
\mathbf{F} \\
\mathbf{F}\end{array}$ & $\begin{array}{l}\mathbf{E} \\
\mathbf{E} \\
\mathbf{E} \\
\mathbf{E} \\
\mathbf{E}\end{array}$ & $\begin{array}{l}\text { A } \\
\text { A } \\
\text { A } \\
\text { A } \\
\text { A }\end{array}$ & $\begin{array}{l}\mathbf{G} \\
\mathbf{G} \\
\mathbf{G} \\
\mathbf{G} \\
\mathbf{F}\end{array}$ & $\begin{array}{l}\mathbf{D} \\
\mathbf{D} \\
\mathbf{D} \\
\mathbf{D} \\
\mathbf{D}\end{array}$ & $\begin{array}{l}\mathbf{E} \\
\mathbf{F} \\
\mathbf{F} \\
\mathbf{F} \\
\mathbf{E}\end{array}$ & $\begin{array}{l}\mathbf{E} \\
\mathbf{E} \\
\mathbf{E} \\
\mathbf{E} \\
\mathbf{E}\end{array}$ & $\begin{array}{l}\mathbf{D} \\
\mathbf{E} \\
\mathbf{E} \\
\mathbf{D} \\
\mathbf{D}\end{array}$ & $\begin{array}{l}\mathbf{E} \\
\mathbf{N} \\
\mathbf{N} \\
\mathbf{E} \\
\mathbf{E}\end{array}$ & $\begin{array}{l}\mathbf{E} \\
\mathbf{E} \\
\mathbf{E} \\
\mathbf{E} \\
\mathbf{E}\end{array}$ & $\Rightarrow$ \\
\hline $\begin{array}{l}P=98 \\
P=97 \\
P=96 \\
P\end{array}$ & $\begin{array}{l}\text { VEM-391-47 } \\
\text { VEM-390-47 } \\
\text { VEM-389-47 } \\
\text { VEM-388-47 } \\
\text { VEM-387-47 }\end{array}$ & $\begin{array}{l}\mathbf{C} \\
\mathbf{C} \\
\mathbf{C} \\
\mathbf{C} \\
\mathbf{C}\end{array}$ & $\begin{array}{l}\mathbf{F} \\
\mathbf{F} \\
\mathbf{F} \\
\mathbf{F}\end{array}$ & $\begin{array}{l}\text { C } \\
\mathbf{C} \\
\mathbf{C} \\
\mathbf{C} \\
\mathbf{A}\end{array}$ & $\begin{array}{l}\mathbf{E} \\
\mathbf{E} \\
\mathbf{E} \\
\mathbf{D} \\
\mathbf{E}\end{array}$ & $\begin{array}{l}\text { ND } \\
\text { ND } \\
\text { ND } \\
\text { ND } \\
\text { ND }\end{array}$ & $\begin{array}{l}\mathbf{G} \\
\mathbf{G} \\
\mathbf{G} \\
\mathbf{G} \\
\mathbf{G}\end{array}$ & $\begin{array}{l}C \\
C \\
C \\
C \\
C\end{array}$ & $\begin{array}{l}\mathbf{D} \\
\mathbf{D} \\
\mathbf{D} \\
\mathbf{D} \\
\mathbf{D}\end{array}$ & $\begin{array}{l}\mathbf{E} \\
\mathbf{E} \\
\mathbf{E} \\
\mathbf{E} \\
\mathbf{E}\end{array}$ & $\begin{array}{l}\mathbf{F} \\
\mathbf{F} \\
\mathbf{F} \\
\mathbf{F} \\
\mathbf{F}\end{array}$ & $\begin{array}{l}\mathbf{E} \\
\mathbf{E} \\
\mathbf{E} \\
\mathbf{E} \\
\mathbf{E}\end{array}$ & $\begin{array}{l}\text { A } \\
\text { A } \\
\text { A } \\
\text { A } \\
\text { A }\end{array}$ & $\begin{array}{l}\mathbf{F} \\
\mathbf{G} \\
\mathbf{G} \\
\mathbf{G} \\
\mathbf{G}\end{array}$ & $\begin{array}{l}\text { D } \\
\text { D } \\
\text { D } \\
\text { D } \\
\text { D }\end{array}$ & $\begin{array}{l}\mathbf{E} \\
\mathbf{F} \\
\mathbf{E} \\
\mathbf{E} \\
\mathbf{D}\end{array}$ & $\begin{array}{l}\mathbf{E} \\
\mathbf{E} \\
\mathbf{E} \\
\mathbf{E} \\
\mathbf{E}\end{array}$ & $\begin{array}{l}\mathbf{D} \\
\mathbf{D} \\
\mathbf{D} \\
\mathbf{D} \\
\mathbf{D}\end{array}$ & $\begin{array}{l}\mathbf{E} \\
\mathbf{E} \\
\mathbf{E} \\
\mathbf{E} \\
\mathbf{E}\end{array}$ & $\begin{array}{l}\mathbf{E} \\
\mathbf{E} \\
\mathbf{E} \\
\mathbf{E} \\
\mathbf{E}\end{array}$ & $\frac{7}{5}$ \\
\hline $\begin{array}{l}P=93 \\
P=92 \\
P=91 \\
P=90 \\
P=89\end{array}$ & $\begin{array}{l}\text { VEM-386-47 } \\
\text { VEM-385-47 } \\
\text { VEM-384-47 } \\
\text { VEM-383-47 } \\
\text { VEM-382-47 }\end{array}$ & $\begin{array}{l}\mathbf{C} \\
\mathbf{C} \\
\mathbf{C} \\
\mathbf{C} \\
\mathbf{C}\end{array}$ & $\begin{array}{l}\mathbf{F} \\
\mathbf{F} \\
\mathbf{F} \\
\mathbf{F}\end{array}$ & $\begin{array}{l}\text { B } \\
\text { A. } \\
\text { B } \\
\text { A } \\
\text { C }\end{array}$ & $\begin{array}{l}\mathbf{E} \\
\mathbf{D} \\
\mathbf{D} \\
\mathbf{E} \\
\mathbf{E}\end{array}$ & $\begin{array}{l}\text { ND } \\
\text { ND } \\
\text { ND } \\
\text { ND } \\
\text { ND }\end{array}$ & $\begin{array}{l}\mathbf{G} \\
\mathbf{G} \\
\mathbf{G} \\
\mathbf{G} \\
\mathbf{G}\end{array}$ & $\begin{array}{l}C \\
C \\
C \\
C \\
C\end{array}$ & $\begin{array}{l}\mathbf{D} \\
\mathbf{D} \\
\mathbf{D} \\
\mathbf{D} \\
\mathbf{D}\end{array}$ & $\begin{array}{l}\mathbf{E} \\
\mathbf{E} \\
\mathbf{E} \\
\mathbf{E} \\
\mathbf{E}\end{array}$ & $\begin{array}{l}\mathbf{F} \\
\mathbf{F} \\
\mathbf{F} \\
\mathbf{F} \\
\mathbf{F}\end{array}$ & $\begin{array}{l}\mathbf{E} \\
\mathbf{E} \\
\mathbf{E} \\
\mathbf{E} \\
\mathbf{E}\end{array}$ & $\begin{array}{l}\text { A } \\
\text { A } \\
\text { A } \\
\text { A } \\
\text { A }\end{array}$ & $\begin{array}{l}\mathbf{G} \\
\mathbf{F} \\
\mathbf{F} \\
\mathbf{F} \\
\mathbf{F}\end{array}$ & $\begin{array}{l}\text { D } \\
\text { D } \\
\text { D } \\
\text { D } \\
\text { D }\end{array}$ & $\begin{array}{l}\mathbf{E} \\
\mathbf{E} \\
\mathbf{E} \\
\mathbf{D} \\
\mathbf{E}\end{array}$ & $\begin{array}{l}\mathbf{E} \\
\mathbf{E} \\
\mathbf{E} \\
\mathbf{E} \\
\mathbf{E}\end{array}$ & $\begin{array}{l}\text { D } \\
\text { D } \\
\text { D } \\
\text { D } \\
\text { D }\end{array}$ & $\begin{array}{l}\mathbf{E} \\
\mathbf{E} \\
\mathbf{E} \\
\mathbf{E} \\
\mathbf{E}\end{array}$ & $\begin{array}{l}\mathbf{E} \\
\mathbf{E} \\
\mathbf{E} \\
\mathbf{E} \\
\mathbf{E}\end{array}$ & $\sum^{\infty}$ \\
\hline 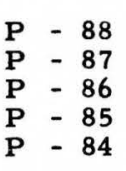 & $\begin{array}{l}\text { VEM-381-47 } \\
\text { VEM-380-47 } \\
\text { VEM-379-47 } \\
\text { VEM-378-47 } \\
\text { VEM-377-47 }\end{array}$ & $\begin{array}{l}\text { C } \\
\mathbf{C} \\
\mathbf{C} \\
\mathbf{C} \\
\mathbf{C}\end{array}$ & $\begin{array}{l}\mathbf{F} \\
\mathbf{F} \\
\mathbf{F} \\
\mathbf{F}\end{array}$ & $\begin{array}{l}\text { B } \\
\text { C } \\
\text { A } \\
\text { C } \\
\text { C }\end{array}$ & $\begin{array}{l}\mathbf{E} \\
\mathbf{E} \\
\mathbf{E} \\
\mathbf{E} \\
\mathbf{E}\end{array}$ & $\begin{array}{l}\text { ND } \\
\text { ND } \\
\text { ND } \\
\text { ND } \\
\text { ND }\end{array}$ & $\begin{array}{l}\mathbf{G} \\
\mathbf{G} \\
\mathbf{G} \\
\mathbf{G} \\
\mathbf{G}\end{array}$ & $\begin{array}{l}\mathrm{C} \\
\mathrm{C} \\
\mathbf{C} \\
\mathbf{C} \\
\mathrm{C}\end{array}$ & $\begin{array}{l}\text { D } \\
\text { D } \\
\text { C } \\
\text { D } \\
\text { D }\end{array}$ & $\begin{array}{l}\mathbf{E} \\
\mathbf{E} \\
\mathbf{E} \\
\mathbf{E} \\
\mathbf{E}\end{array}$ & $\begin{array}{l}\mathbf{F} \\
\mathbf{F} \\
\mathbf{F} \\
\mathbf{F} \\
\mathbf{F}\end{array}$ & $\begin{array}{l}\mathbf{E} \\
\mathbf{E} \\
\mathbf{E} \\
\mathbf{E} \\
\mathbf{E}\end{array}$ & $\begin{array}{l}\text { A } \\
\text { A } \\
\text { A } \\
\text { A } \\
\text { A }\end{array}$ & $\begin{array}{l}\mathbf{G} \\
\mathbf{G} \\
\mathbf{G} \\
\mathbf{G} \\
\mathbf{G}\end{array}$ & $\begin{array}{l}\text { D } \\
\text { D } \\
\text { D } \\
\text { D } \\
\text { D }\end{array}$ & $\begin{array}{l}\mathbf{E} \\
\mathbf{F} \\
\mathbf{F} \\
\mathbf{F} \\
\mathbf{F}\end{array}$ & $\begin{array}{l}\mathbf{E} \\
\mathbf{E} \\
\mathbf{E} \\
\mathbf{E} \\
\mathbf{D}\end{array}$ & $\begin{array}{l}\mathbf{E} \\
\mathbf{E} \\
\mathbf{E} \\
\mathbf{E} \\
\mathbf{E}\end{array}$ & $\begin{array}{l}\text { ND } \\
\text { ND } \\
\text { ND } \\
\text { ND } \\
\text { ND }\end{array}$ & $\begin{array}{l}\mathbf{E} \\
\mathbf{E} \\
\mathbf{E} \\
\mathbf{E} \\
\mathbf{E}\end{array}$ & 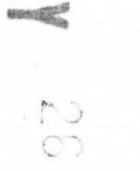 \\
\hline $\begin{array}{l}P=83 \\
P=82 \\
P\end{array}$ & $\begin{array}{l}\text { VEM-376-47 } \\
\text { VEM-375-47 } \\
\text { VEM-374-47 } \\
\text { VEM-373-47 } \\
\text { VEM-372-47 }\end{array}$ & $\begin{array}{l}\mathbf{C} \\
\mathbf{C} \\
\mathbf{C} \\
\mathbf{C} \\
\mathbf{C}\end{array}$ & $\begin{array}{l}\mathbf{F} \\
\mathbf{F} \\
\mathbf{F} \\
\mathbf{F}\end{array}$ & $\begin{array}{l}\text { A } \\
\text { A } \\
\text { B } \\
\text { C } \\
\text { A }\end{array}$ & $\begin{array}{l}\mathbf{E} \\
\mathbf{E} \\
\mathbf{E} \\
\mathbf{E} \\
\mathbf{E}\end{array}$ & $\begin{array}{l}\text { ND } \\
\text { ND } \\
\text { ND } \\
\text { ND } \\
\text { ND }\end{array}$ & $\begin{array}{l}\mathbf{G} \\
\mathbf{G} \\
\mathbf{G} \\
\mathbf{G} \\
\mathbf{G}\end{array}$ & $\begin{array}{l}\mathbf{C} \\
\mathbf{C} \\
\mathbf{C} \\
\mathbf{C} \\
\mathbf{C}\end{array}$ & $\begin{array}{l}\text { C } \\
\text { D } \\
\mathbf{D} \\
\mathbf{D} \\
\mathbf{D}\end{array}$ & $\begin{array}{l}\mathbf{E} \\
\mathbf{E} \\
\mathbf{E} \\
\mathbf{E} \\
\mathbf{E}\end{array}$ & $\begin{array}{l}\mathbf{F} \\
\mathbf{F} \\
\mathbf{F} \\
\mathbf{F} \\
\mathbf{F}\end{array}$ & $\begin{array}{l}\mathbf{E} \\
\mathbf{E} \\
\mathbf{E} \\
\mathbf{E} \\
\mathbf{E}\end{array}$ & $\begin{array}{l}\text { A } \\
\text { A } \\
\text { A } \\
\text { A } \\
\text { B }\end{array}$ & $\begin{array}{l}\mathbf{G} \\
\mathbf{G} \\
\mathbf{G} \\
\mathbf{G} \\
\mathbf{G}\end{array}$ & $\begin{array}{l}\mathbf{D} \\
\mathbf{D} \\
\mathbf{D} \\
\mathbf{D} \\
\mathbf{D}\end{array}$ & $\begin{array}{l}\mathbf{F} \\
\mathbf{E} \\
\mathbf{E} \\
\mathbf{F} \\
\mathbf{D}\end{array}$ & $\begin{array}{l}\mathbf{E} \\
\mathbf{E} \\
\mathbf{E} \\
\mathbf{D} \\
\mathbf{E}\end{array}$ & $\begin{array}{l}\mathbf{E} \\
\mathbf{E} \\
\mathbf{E} \\
\mathbf{E} \\
\mathbf{E}\end{array}$ & $\begin{array}{l}\text { ND } \\
\text { ND } \\
\text { ND } \\
\text { ND } \\
\text { ND }\end{array}$ & $\begin{array}{l}\mathbf{E} \\
\mathbf{E} \\
\mathbf{E} \\
\mathbf{E} \\
\mathbf{E}\end{array}$ & \\
\hline $\begin{array}{l}P=78 \\
P=77 \\
P=76 \\
P=75 \\
P=74\end{array}$ & $\begin{array}{l}\text { VEM-371-47 } \\
\text { VEM-370-47 } \\
\text { VEM-369-47 } \\
\text { VEM-368-47 } \\
\text { VEM-367-47 }\end{array}$ & $\begin{array}{l}\mathbf{C} \\
\mathbf{C} \\
\mathbf{C} \\
\mathbf{C} \\
\mathbf{C}\end{array}$ & $\begin{array}{l}\mathbf{F} \\
\mathbf{F} \\
\mathbf{F} \\
\mathbf{F} \\
\mathbf{F}\end{array}$ & $\begin{array}{l}\text { A } \\
\mathbf{C} \\
\mathbf{A} \\
\mathbf{C} \\
\mathbf{C}\end{array}$ & $\begin{array}{l}\mathbf{E} \\
\mathbf{E} \\
\mathbf{E} \\
\mathbf{E} \\
\mathbf{E}\end{array}$ & $\begin{array}{l}\text { ND } \\
\text { ND } \\
\text { ND } \\
\text { ND } \\
\text { ND }\end{array}$ & $\begin{array}{l}\mathbf{G} \\
\mathbf{G} \\
\mathbf{G} \\
\mathbf{G} \\
\mathbf{G}\end{array}$ & $\begin{array}{l}\mathbf{C} \\
\mathbf{C} \\
\mathbf{C} \\
\mathbf{C} \\
\mathbf{C}\end{array}$ & $\begin{array}{l}\mathbf{D} \\
\mathbf{D} \\
\mathbf{D} \\
\mathbf{D} \\
\mathbf{D}\end{array}$ & $\begin{array}{l}\mathbf{E} \\
\mathbf{E} \\
\mathbf{F} \\
\mathbf{F} \\
\mathbf{E}\end{array}$ & $\begin{array}{l}\mathbf{F} \\
\mathbf{F} \\
\mathbf{E} \\
\mathbf{F} \\
\mathbf{F}\end{array}$ & $\begin{array}{l}\mathbf{E} \\
\mathbf{E} \\
\mathbf{E} \\
\mathbf{E} \\
\mathbf{E}\end{array}$ & $\begin{array}{l}\mathbf{A} \\
\mathbf{A} \\
\mathbf{A} \\
\mathbf{A} \\
\mathbf{A}\end{array}$ & $\begin{array}{l}\mathbf{G} \\
\mathbf{G} \\
\mathbf{G} \\
\mathbf{G} \\
\mathbf{G}\end{array}$ & $\begin{array}{l}\text { D } \\
\text { D } \\
\text { D } \\
\text { D } \\
\text { D }\end{array}$ & $\begin{array}{l}\mathbf{D} \\
\mathbf{F} \\
\mathbf{E} \\
\mathbf{F} \\
\mathbf{F}\end{array}$ & $\begin{array}{l}\mathbf{E} \\
\mathbf{D} \\
\mathbf{E} \\
\mathbf{E} \\
\mathbf{E}\end{array}$ & $\begin{array}{l}\mathbf{E} \\
\mathbf{E} \\
\mathbf{E} \\
\mathbf{E} \\
\mathbf{D}\end{array}$ & $\begin{array}{l}\text { ND } \\
\text { ND } \\
\text { ND } \\
\text { ND } \\
\text { ND }\end{array}$ & $\begin{array}{l}\mathbf{E} \\
\mathbf{E} \\
\mathbf{E} \\
\mathbf{E} \\
\mathbf{E}\end{array}$ & \\
\hline $\begin{array}{l}P=73 \\
P=72 \\
P=71 \\
P=70 \\
P=69\end{array}$ & $\begin{array}{l}\text { VEM-366-47 } \\
\text { VEM-365-47 } \\
\text { VEM-364-47 } \\
\text { VEM-363-47 } \\
\text { VEM-362-47 }\end{array}$ & $\begin{array}{l}\mathbf{C} \\
\mathbf{C} \\
\mathbf{C} \\
\mathbf{C} \\
\mathbf{C}\end{array}$ & $\begin{array}{l}\mathbf{F} \\
\mathbf{F} \\
\mathbf{F} \\
\mathbf{F} \\
\mathbf{F}\end{array}$ & $\begin{array}{l}\mathbf{A} \\
\mathbf{C} \\
\mathbf{C} \\
\mathbf{A} \\
\mathbf{A}\end{array}$ & $\begin{array}{l}\mathbf{D} \\
\mathbf{E} \\
\mathbf{E} \\
\mathbf{D} \\
\mathbf{E}\end{array}$ & $\begin{array}{l}\text { ND } \\
\text { ND } \\
\text { ND } \\
\text { ND } \\
\text { ND }\end{array}$ & $\begin{array}{l}\mathbf{G} \\
\mathbf{G} \\
\mathbf{G} \\
\mathbf{G} \\
\mathbf{G}\end{array}$ & $\begin{array}{l}\mathbf{C} \\
\mathbf{C} \\
\mathbf{C} \\
\mathbf{C} \\
\mathbf{C}\end{array}$ & $\begin{array}{l}\text { D } \\
\text { D } \\
\text { D } \\
\text { D } \\
\text { D }\end{array}$ & $\begin{array}{l}\mathbf{F} \\
\mathbf{E} \\
\mathbf{E} \\
\mathbf{F} \\
\mathbf{F}\end{array}$ & $\begin{array}{l}\mathbf{F} \\
\mathbf{F} \\
\mathbf{F} \\
\mathbf{F} \\
\mathbf{F}\end{array}$ & $\begin{array}{l}\mathbf{E} \\
\mathbf{E} \\
\mathbf{E} \\
\mathbf{E} \\
\mathbf{E}\end{array}$ & $\begin{array}{l}\text { A } \\
\text { A } \\
\text { A } \\
\text { A } \\
\text { C }\end{array}$ & $\begin{array}{l}\mathbf{G} \\
\mathbf{G} \\
\mathbf{G} \\
\mathbf{G} \\
\mathbf{G}\end{array}$ & $\begin{array}{l}\mathbf{D} \\
\mathbf{D} \\
\mathbf{D} \\
\mathbf{E} \\
\mathbf{E}\end{array}$ & $\begin{array}{l}\mathbf{E} \\
\mathbf{F} \\
\mathbf{F} \\
\mathbf{E} \\
\mathbf{E}\end{array}$ & $\begin{array}{l}\mathbf{E} \\
\mathbf{D} \\
\mathbf{D} \\
\mathbf{E} \\
\mathbf{E}\end{array}$ & $\begin{array}{l}\mathbf{E} \\
\mathbf{E} \\
\mathbf{E} \\
\mathbf{E} \\
\mathbf{E}\end{array}$ & $\begin{array}{l}\text { ND } \\
\text { ND } \\
\text { ND } \\
\text { E } \\
\text { E }\end{array}$ & $\begin{array}{l}\mathbf{E} \\
\mathbf{E} \\
\mathbf{E} \\
\mathbf{E} \\
\mathbf{E}\end{array}$ & \\
\hline $\begin{array}{l}P=68 \\
P=67 \\
P=66 \\
P=65 \\
P=64\end{array}$ & $\begin{array}{l}\text { VEM-361-47 } \\
\text { VEM-360-47 } \\
\text { VEM-359-47 } \\
\text { VEM-344-47 } \\
\text { VEM-343-47 }\end{array}$ & $\begin{array}{l}\mathbf{C} \\
\mathbf{C} \\
\mathbf{C} \\
\mathbf{C} \\
--\end{array}$ & $\begin{array}{l}\mathbf{F} \\
\mathbf{F} \\
\mathbf{F} \\
\mathbf{F} \\
--\end{array}$ & $\begin{array}{l}\mathbf{A} \\
\mathbf{A} \\
\mathbf{B} \\
\mathbf{B} \\
--\end{array}$ & $\begin{array}{l}\mathbf{E} \\
\mathbf{E} \\
\mathbf{E} \\
\mathbf{D} \\
--\end{array}$ & $\begin{array}{l}\text { ND } \\
\text { ND } \\
\text { ND } \\
\text { ND } \\
--\end{array}$ & $\begin{array}{l}\mathbf{G} \\
\mathbf{G} \\
\mathbf{G} \\
\mathbf{G} \\
--\end{array}$ & $\begin{array}{l}\mathbf{C} \\
\mathbf{C} \\
\mathbf{C} \\
\mathbf{C} \\
--\end{array}$ & $\begin{array}{l}\mathbf{D} \\
\mathbf{D} \\
\mathbf{D} \\
\mathbf{D} \\
--\end{array}$ & $\begin{array}{l}\mathbf{E} \\
\mathbf{E} \\
\mathbf{E} \\
\mathbf{E} \\
--\end{array}$ & $\begin{array}{l}\mathbf{F} \\
\mathbf{F} \\
\mathbf{F} \\
\mathbf{F} \\
--\end{array}$ & $\begin{array}{l}\mathbf{E} \\
\mathbf{E} \\
\mathbf{E} \\
\mathbf{E} \\
--\end{array}$ & $\begin{array}{c}\mathbf{A} \\
\mathbf{A} \\
\mathbf{A} \\
\mathbf{A} \\
--\end{array}$ & $\begin{array}{l}\mathbf{G} \\
\mathbf{G} \\
\mathbf{F} \\
\mathbf{F} \\
-\end{array}$ & $\begin{array}{l}\mathbf{D} \\
\mathbf{D} \\
\mathbf{D} \\
\mathbf{E} \\
--\end{array}$ & $\begin{array}{l}\mathbf{E} \\
\mathbf{E} \\
\mathbf{D} \\
\mathbf{E} \\
--\end{array}$ & $\begin{array}{l}\mathbf{E} \\
\mathbf{E} \\
\mathbf{E} \\
\mathbf{E} \\
--\end{array}$ & $\begin{array}{l}\mathbf{E} \\
\mathbf{E} \\
\mathbf{E} \\
\mathbf{D} \\
--\end{array}$ & $\begin{array}{l}\mathbf{E} \\
\mathbf{E} \\
\mathbf{E} \\
\mathbf{E} \\
--\end{array}$ & $\begin{array}{l}\mathbf{E} \\
\mathbf{E} \\
\mathbf{E} \\
\mathbf{E} \\
\cdots-\end{array}$ & \\
\hline$P-63$ & VEM-342-47 & C & $\mathbf{F}$ & A & D & ND & G & C & C & $\mathbf{E}$ & $\mathbf{E}$ & $\mathbf{E}$ & A & $\mathbf{G}$ & $\mathbf{D}$ & $\mathbf{E}$ & $\mathbf{E}$ & D & $\mathbf{E}$ & E & \\
\hline
\end{tabular}




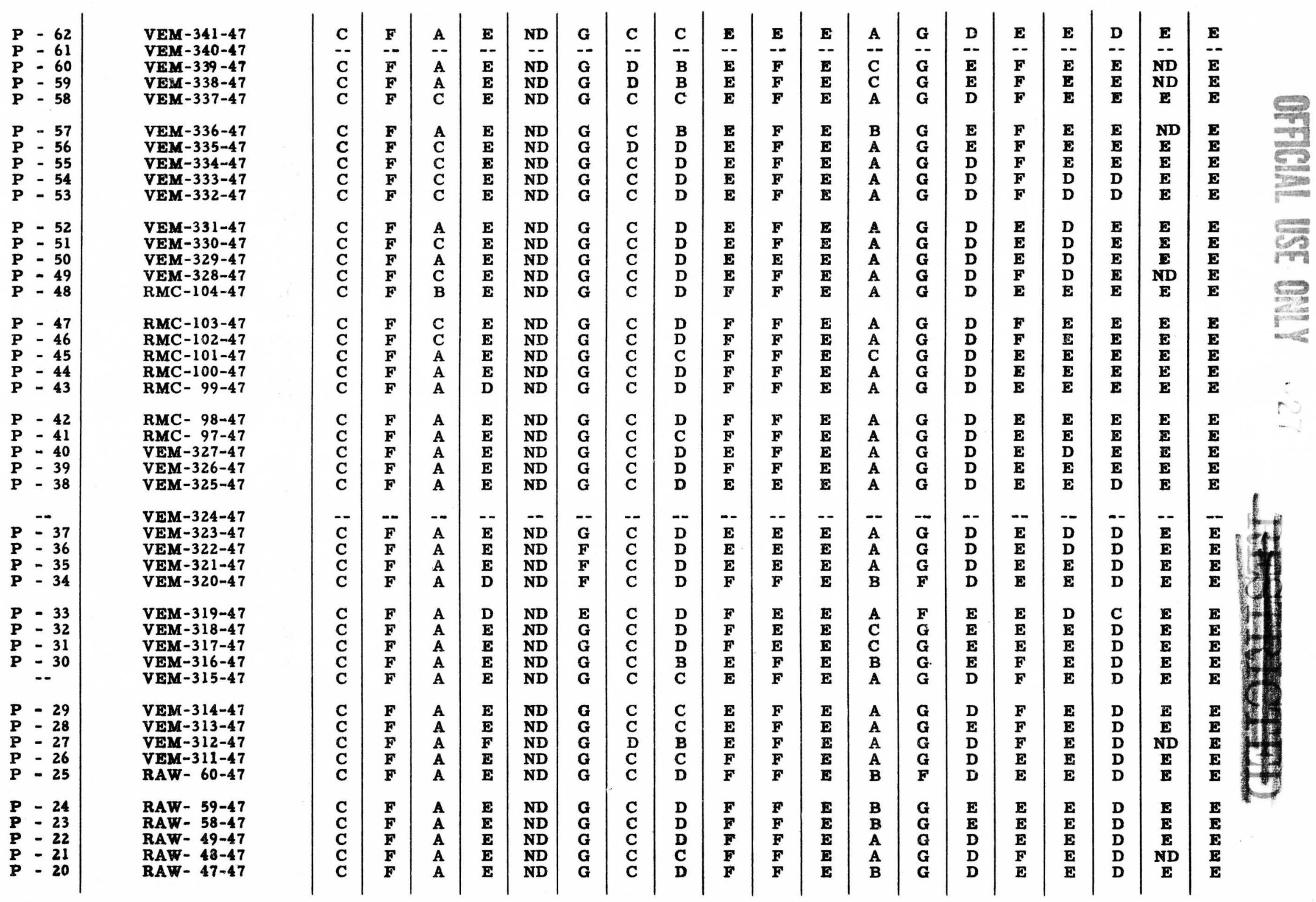




\begin{tabular}{|c|c|c|c|c|c|c|c|c|c|c|c|c|c|c|c|c|c|c|c|c|c|}
\hline & $\begin{array}{l}\text { Bed } \\
\text { no. }\end{array}$ & Sample no. & Al & B & $\mathbf{C a}$ & $\mathbf{C r}$ & Co & $\mathbf{C u}$ & $\mathbf{F e}$ & Mg & $\mathbf{M n}$ & Mo & $\mathbf{N i}$ & Si & Ag & $\mathbf{N a}$ & Sr & $\mathbf{T i}$ & $\mathbf{v}$ & $\mathbf{Z n}$ & $\mathbf{Z r}$ \\
\hline $\begin{array}{l}\mathbf{P} \\
\mathbf{P} \\
\mathbf{P} \\
\mathbf{P}\end{array}$ & $\begin{array}{l}-19 \\
-- \\
-18 \\
-17 \\
-16\end{array}$ & $\begin{array}{l}\text { RAW- } 46-47 \\
\text { RAW- } 45-47 \\
\text { RAW- } 44-47 \\
\text { RAW- } 43-47 \\
\text { RAW- } 42-47\end{array}$ & $\begin{array}{l}\mathbf{C} \\
\mathbf{C} \\
\mathbf{C} \\
\mathbf{C} \\
\mathbf{C}\end{array}$ & $\begin{array}{l}\mathbf{F} \\
\mathbf{F} \\
\mathbf{F} \\
\mathbf{F} \\
\mathbf{F}\end{array}$ & $\begin{array}{l}\mathbf{A} \\
\mathbf{A} \\
\mathbf{A} \\
\mathbf{A} \\
\mathbf{A}\end{array}$ & $\begin{array}{l}\mathbf{E} \\
\mathbf{E} \\
\mathbf{E} \\
\mathbf{E} \\
\mathbf{E}\end{array}$ & $\begin{array}{l}\text { ND } \\
\text { ND } \\
\text { ND } \\
\text { ND } \\
\text { ND }\end{array}$ & $\begin{array}{l}\mathbf{G} \\
\mathbf{G} \\
\mathbf{G} \\
\mathbf{G} \\
\mathbf{G}\end{array}$ & $\begin{array}{l}\mathbf{C} \\
\mathbf{D} \\
\mathbf{C} \\
\mathbf{C} \\
\mathbf{D}\end{array}$ & $\begin{array}{l}\mathbf{D} \\
\mathbf{D} \\
\mathbf{D} \\
\mathbf{D} \\
\mathbf{C}\end{array}$ & $\begin{array}{l}\mathbf{F} \\
\mathbf{F} \\
\mathbf{F} \\
\mathbf{F} \\
\mathbf{F}\end{array}$ & $\begin{array}{l}\mathbf{F} \\
\mathbf{F} \\
\mathbf{F} \\
\mathbf{F} \\
\mathbf{F}\end{array}$ & $\begin{array}{l}\mathbf{E} \\
\mathbf{E} \\
\mathbf{E} \\
\mathbf{E} \\
\mathbf{E}\end{array}$ & $\begin{array}{l}\mathbf{A} \\
\mathbf{C} \\
\mathbf{A} \\
\mathbf{A} \\
\mathbf{C}\end{array}$ & $\begin{array}{l}\mathbf{F} \\
\mathbf{G} \\
\mathbf{F} \\
\mathbf{F} \\
\mathbf{G}\end{array}$ & $\begin{array}{l}\mathbf{D} \\
\mathbf{E} \\
\mathbf{D} \\
\mathbf{D} \\
\mathbf{E}\end{array}$ & $\begin{array}{l}\mathbf{E} \\
\mathbf{F} \\
\mathbf{E} \\
\mathbf{E} \\
\mathbf{F}\end{array}$ & $\begin{array}{l}\mathbf{E} \\
\mathbf{E} \\
\mathbf{E} \\
\mathbf{E} \\
\mathbf{E}\end{array}$ & $\begin{array}{l}\mathbf{D} \\
\mathbf{E} \\
\mathbf{D} \\
\mathbf{D} \\
\mathbf{D}\end{array}$ & $\begin{array}{c}\mathbf{E} \\
\mathbf{N D} \\
\mathbf{E} \\
\mathbf{E} \\
\mathbf{E}\end{array}$ & $\begin{array}{l}\mathbf{E} \\
\mathbf{E} \\
\mathbf{E} \\
\mathbf{E} \\
\mathbf{E}\end{array}$ \\
\hline $\begin{array}{l}\mathbf{P} \\
\mathbf{P} \\
\mathbf{P} \\
\mathbf{P} \\
\mathbf{P}\end{array}$ & $\begin{array}{l}-15 \\
-14 \\
-13 \\
-12 \\
-11\end{array}$ & $\begin{array}{l}\text { RAW- } 41-47 \\
\text { RAW- } 40-47 \\
\text { RAW- } 39-47 \\
\text { RAW- } 38-47 \\
\text { RAW- } 37-47\end{array}$ & $\begin{array}{l}\mathbf{C} \\
\mathbf{C} \\
\mathbf{C} \\
\mathbf{C} \\
\mathbf{C}\end{array}$ & $\begin{array}{l}\mathbf{F} \\
\mathbf{F} \\
\mathbf{F} \\
\mathbf{F} \\
\mathbf{F}\end{array}$ & $\begin{array}{l}\text { A } \\
\text { A } \\
\text { A } \\
\text { A } \\
\text { A }\end{array}$ & $\begin{array}{l}\mathbf{E} \\
\mathbf{E} \\
\mathbf{E} \\
\mathbf{E} \\
\mathbf{E}\end{array}$ & $\begin{array}{l}\text { ND } \\
\text { ND } \\
\text { ND } \\
\text { ND } \\
\text { ND }\end{array}$ & $\begin{array}{l}\mathbf{G} \\
\mathbf{G} \\
\mathbf{G} \\
\mathbf{G} \\
\mathbf{G}\end{array}$ & $\begin{array}{l}\text { D } \\
\text { C } \\
\text { C } \\
\text { C } \\
\text { D }\end{array}$ & $\begin{array}{l}\text { C } \\
\text { D } \\
\text { C } \\
\text { C } \\
\text { D }\end{array}$ & $\begin{array}{l}\mathbf{F} \\
\mathbf{F} \\
\mathbf{F} \\
\mathbf{F} \\
\mathbf{F}\end{array}$ & $\begin{array}{l}\mathbf{F} \\
\mathbf{F} \\
\mathbf{F} \\
\mathbf{F} \\
\mathbf{F}\end{array}$ & $\begin{array}{l}\mathbf{E} \\
\mathbf{E} \\
\mathbf{E} \\
\mathbf{E} \\
\mathbf{E}\end{array}$ & $\begin{array}{l}\text { C } \\
\text { A } \\
\text { B } \\
\text { C } \\
\text { C }\end{array}$ & $\begin{array}{l}\mathbf{G} \\
\mathbf{F} \\
\mathbf{F} \\
\mathbf{G} \\
\mathbf{G}\end{array}$ & $\begin{array}{l}\mathbf{E} \\
\mathbf{D} \\
\mathbf{D} \\
\mathbf{D} \\
\mathrm{D}\end{array}$ & $\begin{array}{l}\mathbf{F} \\
\mathbf{E} \\
\mathbf{F} \\
\mathbf{F} \\
\mathbf{E}\end{array}$ & $\begin{array}{l}\mathbf{E} \\
\mathbf{E} \\
\mathbf{E} \\
\mathbf{E} \\
\mathbf{E}\end{array}$ & $\begin{array}{l}\mathbf{D} \\
\mathbf{D} \\
\mathbf{D} \\
\mathbf{D} \\
\mathbf{D}\end{array}$ & $\begin{array}{l}\mathbf{E} \\
\mathbf{E} \\
\mathbf{E} \\
\mathbf{E} \\
\mathbf{E}\end{array}$ & $\begin{array}{l}\mathbf{E} \\
\mathbf{E} \\
\mathbf{E} \\
\mathbf{E} \\
\mathbf{E}\end{array}$ \\
\hline $\begin{array}{l}\mathbf{P} \\
\mathbf{P} \\
\mathbf{P} \\
\mathbf{P} \\
\mathbf{P}\end{array}$ & $\begin{array}{l}-10 \\
-\quad 9 \\
-\quad 8 \\
-\quad 7 \\
-\quad 6\end{array}$ & $\begin{array}{l}\text { RAW- } 36-47 \\
\text { RAW- } 35-47 \\
\text { RAW- } 34-47 \\
\text { RAW- } 33-47 \\
\text { RAW- } 32-47\end{array}$ & $\begin{array}{l}\mathbf{C} \\
\mathbf{C} \\
\mathbf{C} \\
\mathbf{C} \\
\mathbf{C}\end{array}$ & $\begin{array}{l}\mathbf{F} \\
\mathbf{F} \\
\mathbf{F} \\
\mathbf{F} \\
\mathbf{F}\end{array}$ & $\begin{array}{l}\text { A } \\
\text { A } \\
\text { A } \\
\text { A } \\
\text { A }\end{array}$ & $\begin{array}{l}\mathbf{E} \\
\mathbf{E} \\
\mathbf{E} \\
\mathbf{E} \\
\mathbf{E}\end{array}$ & $\begin{array}{l}\text { ND } \\
\text { ND } \\
\text { ND } \\
\text { ND } \\
\text { ND }\end{array}$ & $\begin{array}{l}\mathbf{G} \\
\mathbf{G} \\
\mathbf{G} \\
\mathbf{G} \\
\mathbf{G}\end{array}$ & $\begin{array}{l}\mathbf{C} \\
\mathbf{C} \\
\mathbf{C} \\
\mathbf{C} \\
\mathbf{C}\end{array}$ & $\begin{array}{l}\text { D } \\
\text { D } \\
\text { D } \\
\text { D } \\
\text { D }\end{array}$ & $\begin{array}{l}\mathbf{F} \\
\mathbf{F} \\
\mathbf{F} \\
\mathbf{F} \\
\mathbf{F}\end{array}$ & $\begin{array}{l}\mathbf{F} \\
\mathbf{F} \\
\mathbf{F} \\
\mathbf{F} \\
\mathbf{F}\end{array}$ & $\begin{array}{l}\mathbf{E} \\
\mathbf{E} \\
\mathbf{E} \\
\mathbf{E} \\
\mathbf{E}\end{array}$ & $\begin{array}{l}\text { C } \\
\mathbf{C} \\
\mathbf{C} \\
\mathrm{C} \\
\mathrm{C}\end{array}$ & $\begin{array}{l}\mathbf{G} \\
\mathbf{G} \\
\mathbf{G} \\
\mathbf{G} \\
\mathbf{G}\end{array}$ & $\begin{array}{l}\text { D } \\
\text { D } \\
D \\
D \\
D\end{array}$ & $\begin{array}{l}\mathbf{E} \\
\mathbf{E} \\
\mathbf{E} \\
\mathbf{E} \\
\mathbf{E}\end{array}$ & $\begin{array}{l}\mathbf{E} \\
\mathbf{E} \\
\mathbf{E} \\
\mathbf{E} \\
\mathbf{E}\end{array}$ & $\begin{array}{l}\mathbf{D} \\
\mathbf{D} \\
\mathbf{D} \\
\mathbf{D} \\
\mathbf{D}\end{array}$ & $\begin{array}{l}\mathbf{E} \\
\mathbf{E} \\
\mathbf{E} \\
\mathbf{E} \\
\mathbf{E}\end{array}$ & $\begin{array}{l}\mathbf{E} \\
\mathbf{E} \\
\mathbf{E} \\
\mathbf{E} \\
\mathbf{E}\end{array}$ \\
\hline $\begin{array}{l}\mathbf{P} \\
\mathbf{P} \\
\mathbf{P} \\
\mathbf{P} \\
\mathbf{P}\end{array}$ & $\begin{array}{l}-5 \\
-\quad 4 \\
-\quad 3 \\
-\quad 2 \\
-\quad 1\end{array}$ & $\begin{array}{l}\text { RAW- } 31-47 \\
\text { RAW- } 30-47 \\
\text { RAW- } 29-47 \\
\text { RAW- } 28-47 \\
\text { RAW- } 61-47\end{array}$ & $\begin{array}{l}\mathbf{C} \\
\mathbf{C} \\
\mathbf{C} \\
\mathbf{C} \\
\mathbf{C}\end{array}$ & $\begin{array}{l}\mathbf{F} \\
\mathbf{F} \\
\mathbf{F} \\
\mathbf{F} \\
\mathbf{F}\end{array}$ & $\begin{array}{l}\text { C } \\
\text { A } \\
\text { C } \\
\text { A } \\
\text { A }\end{array}$ & $\begin{array}{l}\mathbf{E} \\
\mathbf{E} \\
\mathbf{E} \\
\mathbf{E} \\
\mathbf{E}\end{array}$ & $\begin{array}{l}\text { ND } \\
\text { ND } \\
\text { ND } \\
\text { ND } \\
\text { ND }\end{array}$ & $\begin{array}{l}\mathbf{G} \\
\mathbf{G} \\
\mathbf{G} \\
\mathbf{G} \\
\mathbf{G}\end{array}$ & $\begin{array}{l}\mathbf{C} \\
\mathbf{C} \\
\mathbf{C} \\
\mathbf{C} \\
\mathbf{C}\end{array}$ & $\begin{array}{l}\mathbf{D} \\
\mathbf{C} \\
\mathbf{C} \\
\mathbf{D} \\
\mathbf{D}\end{array}$ & $\begin{array}{l}\mathbf{F} \\
\mathbf{E} \\
\mathbf{E} \\
\mathbf{E} \\
\mathbf{F}\end{array}$ & $\begin{array}{l}\mathbf{F} \\
\mathbf{F} \\
\mathbf{F} \\
\mathbf{F} \\
\mathbf{F}\end{array}$ & $\begin{array}{l}\mathbf{E} \\
\mathbf{E} \\
\mathbf{E} \\
\mathbf{E} \\
\mathbf{E}\end{array}$ & $\begin{array}{l}\mathbf{A} \\
\mathbf{A} \\
\mathbf{A} \\
\mathbf{A} \\
\mathbf{C}\end{array}$ & $\begin{array}{l}\mathbf{G} \\
\mathbf{G} \\
\mathbf{G} \\
\mathbf{G} \\
\mathbf{G}\end{array}$ & $\begin{array}{l}\mathbf{E} \\
\mathbf{E} \\
\mathbf{E} \\
\mathbf{D} \\
\mathbf{D}\end{array}$ & $\begin{array}{l}\mathbf{F} \\
\mathbf{F} \\
\mathbf{F} \\
\mathbf{F} \\
\mathbf{E}\end{array}$ & $\begin{array}{l}\mathbf{E} \\
\mathbf{E} \\
\mathbf{E} \\
\mathbf{E} \\
\mathbf{E}\end{array}$ & $\begin{array}{l}\mathbf{D} \\
\mathbf{D} \\
\mathbf{D} \\
\mathbf{D} \\
\mathbf{E}\end{array}$ & $\begin{array}{l}\mathbf{E} \\
\mathbf{E} \\
\mathbf{E} \\
\mathbf{E} \\
\mathbf{E}\end{array}$ & $\begin{array}{l}\mathbf{E} \\
\mathbf{E} \\
\mathbf{E} \\
\mathbf{E} \\
\mathbf{E}\end{array}$ \\
\hline
\end{tabular}


CONDA, IDAHO. LOT NO. 1200 .

Phosphatic shale member of Phosphoria formation sampled in 300 level east crosscut of Conda mine, sec. 13, T. 8 S., R. 42 E., Caribou County Idaho, on west limb of Trall aynclino. Beds strike $\mathrm{N}$. $10^{\circ} \mathrm{W}$. and dip $55^{\circ}$ E. Section measured by F. C. Armstrong, R. A. Hoppin, and L. E. Smith and sampled by R. A. Gulbrandsen, O. A. Payne, R. S. Sears, and R. P. Sheldon in July 1947. Samples analyzed by U. S. Bureau of Mines Laboratory, Albany, Oregon.

\begin{tabular}{|c|c|c|c|c|c|c|c|c|c|c|c|c|c|}
\hline \multirow{2}{*}{$\begin{array}{c}\text { Bed } \\
\text { no. }\end{array}$} & \multirow{2}{*}{ Rock description } & \multirow{2}{*}{$\begin{array}{c}\text { Sample } \\
\text { no. }\end{array}$} & \multirow{2}{*}{$\begin{array}{l}\text { Thi ckness } \\
\text { (foot) }\end{array}$} & \multicolumn{6}{|c|}{ Chemical analyses (percent) } & \multirow{2}{*}{$\begin{array}{l}\text { Cumulative } \\
\text { thickness } \\
\text { (feet) }\end{array}$} & \multirow{2}{*}{$\begin{array}{l}\text { Thickness } x^{x} \\
\text { percent } \mathbf{P}_{2} \mathbf{O}_{5} \\
\text { (cumulative) }\end{array}$} & \multirow{2}{*}{$\begin{array}{l}\begin{array}{c}\text { Oranium content } \\
\text { (percent) }\end{array} \\
\text { of } \\
\text { of Chem. U }\end{array}$} & \multirow{2}{*}{$\begin{array}{c}\text { Thickness } x \\
\text { percent chem.j } U \\
\text { (cumulative) }\end{array}$} \\
\hline & & & & $\mathrm{P}_{2} \mathrm{O}_{5}$ & $\mathrm{Al}_{2} \mathrm{O}_{3}$ & $\mathrm{Fe}_{2} \mathrm{O}_{3}$ & $\mathrm{v}_{2} \mathrm{O}_{5}$ & $\begin{array}{l}\text { Loss on } \\
\text { ignition }\end{array}$ & $\begin{array}{l}\text { Acrd } \\
\text { Insoluble }\end{array}$ & & & & \\
\hline
\end{tabular}

Rex member of Phosphoria formation-basal beds only

\begin{tabular}{|c|c|c|c|c|c|c|c|c|c|c|c|c|c|c|}
\hline $\begin{array}{l}R=4 \\
R=3 \\
R=2 \\
R=1\end{array}$ & $\begin{array}{l}\text { Chert and mudstone } \\
\text { Limestone, cherty } \\
\text { Chert, calcareous } \\
\text { Mudstone, calcareous }\end{array}$ & $\begin{array}{c}\because \\
\because \\
= \\
\text { FCA }-141-47\end{array}$ & $\begin{array}{l}2.7 \\
1.75 \\
3.0 \\
0.3\end{array}$ & $\begin{array}{l}\because- \\
\because- \\
1.3\end{array}$ & $\begin{array}{l}-- \\
-- \\
5.5\end{array}$ & $\begin{array}{l}-- \\
-- \\
2.4\end{array}$ & $\begin{array}{l}\because- \\
\because- \\
.045\end{array}$ & \begin{tabular}{l}
$=-$ \\
\hdashline- \\
8.6
\end{tabular} & $\begin{array}{c}\because- \\
\ddot{-} \\
66.3\end{array}$ & $\begin{array}{l}2.70 \\
4.45 \\
7.45 \\
7.75\end{array}$ & $\begin{array}{l}\because- \\
\because- \\
0.39\end{array}$ & $\begin{array}{l}\because \\
\ddot{003}\end{array}$ & $\begin{array}{l}-- \\
-\overline{-} \\
.003\end{array}$ & $\begin{array}{l}-- \\
-- \\
--\end{array}$ \\
\hline
\end{tabular}

Phosphatic shale member of Phosphoria formation

\begin{tabular}{l|l}
\hline P -142 & Phopphate rock, calcareous; fos. \\
col, no, 47-HW-12
\end{tabular}

$P$-141 Mudstons, calcarrous

Mudstone, calcareous; fos. col.

$\begin{array}{lll}P & -139 & \text { Mudstone, calcareous } \\ \text { P } & -138 & \text { Mudstono, calcareous; fos. col. }\end{array}$ no. 47-EW-10

P -137 Limestope, arelliaceous; fos. col.

$P$-136 Phosphate rock, argillaceous

P - 135 Phosphato rock

P -134 Mudstone, calcareous

Phosphate rock, calcareou

\begin{tabular}{ll|l}
$P$ & -132 & Phoophate rock \\
$P$ & -131 & Phoephate rock
\end{tabular}

P -130 Phosphate rook

P -129 Phouphate rock

P -127 Phoophate rock

Phosphate rock, calcareous near

P -125 Phosphato rook

\begin{tabular}{ll|l}
$P$ & -124 & Limostone, phosphatic \\
P & -123 & Phosphate rock, calcareous
\end{tabular}

FCA-125-47
FCA-124-47

1 Fosall collection by H. Wedow, Paleontology and Stratigraphy Branch, U. S. Geological Survey.

\begin{tabular}{|l|l|r|r|r|r|r|r|} 
FCA-140-47 & 0.8 & 24.0 & 1.8 & 1.3 & .025 & 16.2 & 15.1 \\
FCA-139-47 & 0.35 & 4.2 & 9.4 & 3.2 & .095 & 13.2 & 54.4 \\
FCA-138-47 & 1.7 & 1.7 & 8.9 & 3.9 & .095 & 13.2 & 60.3 \\
FCA-137-47 & 0.75 & 5.6 & 9.6 & 3.1 & .03 & 15.8 & 51.5 \\
FCA-144-47 & 4.9 & 1.2 & 8.6 & 4.0 & .025 & 24.8 & 58.6 \\
& & & & & & & \\
FCA-143-47 & 2.8 & 4.1 & 4.1 & 1.9 & .015 & 13.5 & 31.6 \\
FCA-142-47 & 0.25 & 24.6 & 5.1 & 2.3 & .03 .5 & 24.8 & 20.3 \\
FCA-136-47 & 1.35 & 28.8 & 2.1 & 1.3 & .08 & 8.4 & 12.3 \\
FCA-135-47 & 0.35 & 4.6 & 8.6 & 3.2 & .17 & 15.8 & 54.0 \\
FCA-134-47 & 0.65 & 25.0 & 0.7 & 0.8 & .035 & 15.3 & 5.1 \\
FCA-133-37 & 0.35 & 34.2 & 0.4 & 0.6 & .06 & 8.5 & 2.3 \\
FCA-132-47 & 0.55 & 26.9 & 2.2 & 1.1 & .14 & 13.5 & 9.9 \\
FCA-131-47 & 0.85 & 33.4 & 0.5 & 0.6 & .065 & 8.8 & 2.6 \\
FCA-130-47 & 0.3 & 30.1 & 1.9 & 1.4 & .06 & 7.7 & 11.3 \\
FCA-129-47 & 0.8 & 22.3 & 1.8 & 1.2 & .04 & 13.8 & 14.3 \\
FCA-128-47 & 0.75 & 32.4 & 1.0 & 1.0 & .09 & 7.4 & 5.2 \\
FCA-127-47 & 0.75 & 26.9 & 2.4 & 1.5 & .09 & 8.7 & 15.0 \\
FCA-126-47 & 0.35 & 26.5 & 2.5 & 1.6 & .13 & 9.6 & 15.3 \\
FCA-125-47 & 0.65 & 7.8 & 1.5 & 0.7 & .03 & 31.0 & 11.7 \\
FCA-124-47 & 0.8 & 24.2 & 1.4 & 0.8 & .08 & 15.8 & 6.9
\end{tabular}

0.80
1.15
2.85
3.60
8.50

11.30
11.55
12.90
13.25
13.90
14.25
14.80
15.65
15.95
16.75
17.50
18.25
18.60
19.25
20.05

19.20
20.67
23.56
27.76
33.64

45.12
51.27
90.15
91.76
108.01
119.98
134.78
163.17
172.20
190.04
214.34
234.52
243.80
248.87
268.23

\begin{tabular}{l|l}
.010 & .007 \\
.004 & .002 \\
.003 & .001 \\
.006 & .005 \\
.002 & .001 \\
& \\
.002 & .001 \\
.012 & .005 \\
.011 & .007 \\
.004 & .002 \\
.007 & .003 \\
.011 & .013 \\
.009 & .009 \\
.009 & .006 \\
.008 & .005 \\
.007 & .005 \\
.011 & .007 \\
.009 & .006 \\
.0012 & .004 \\
.003 & .002 \\
.007 & .006
\end{tabular}




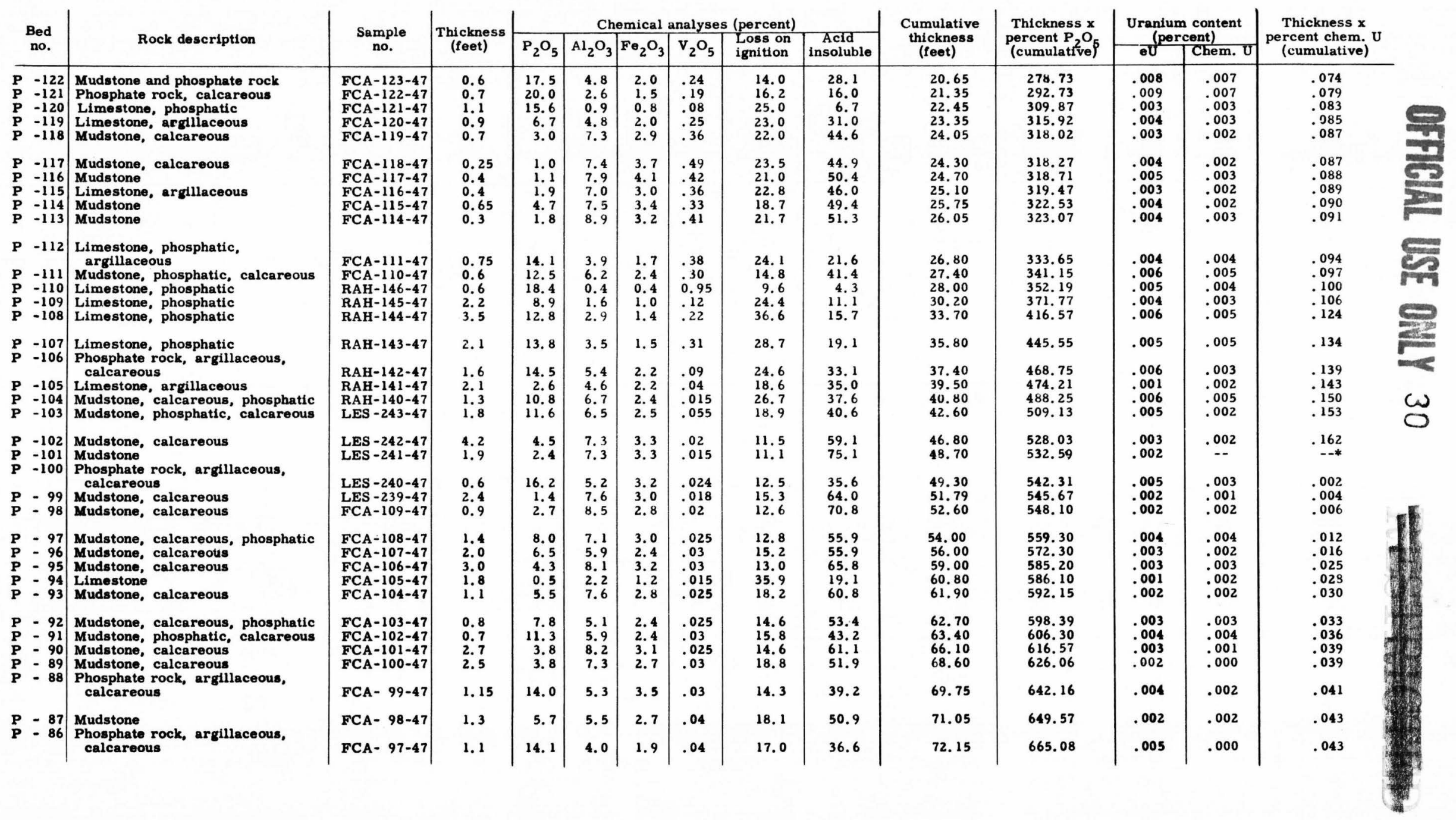




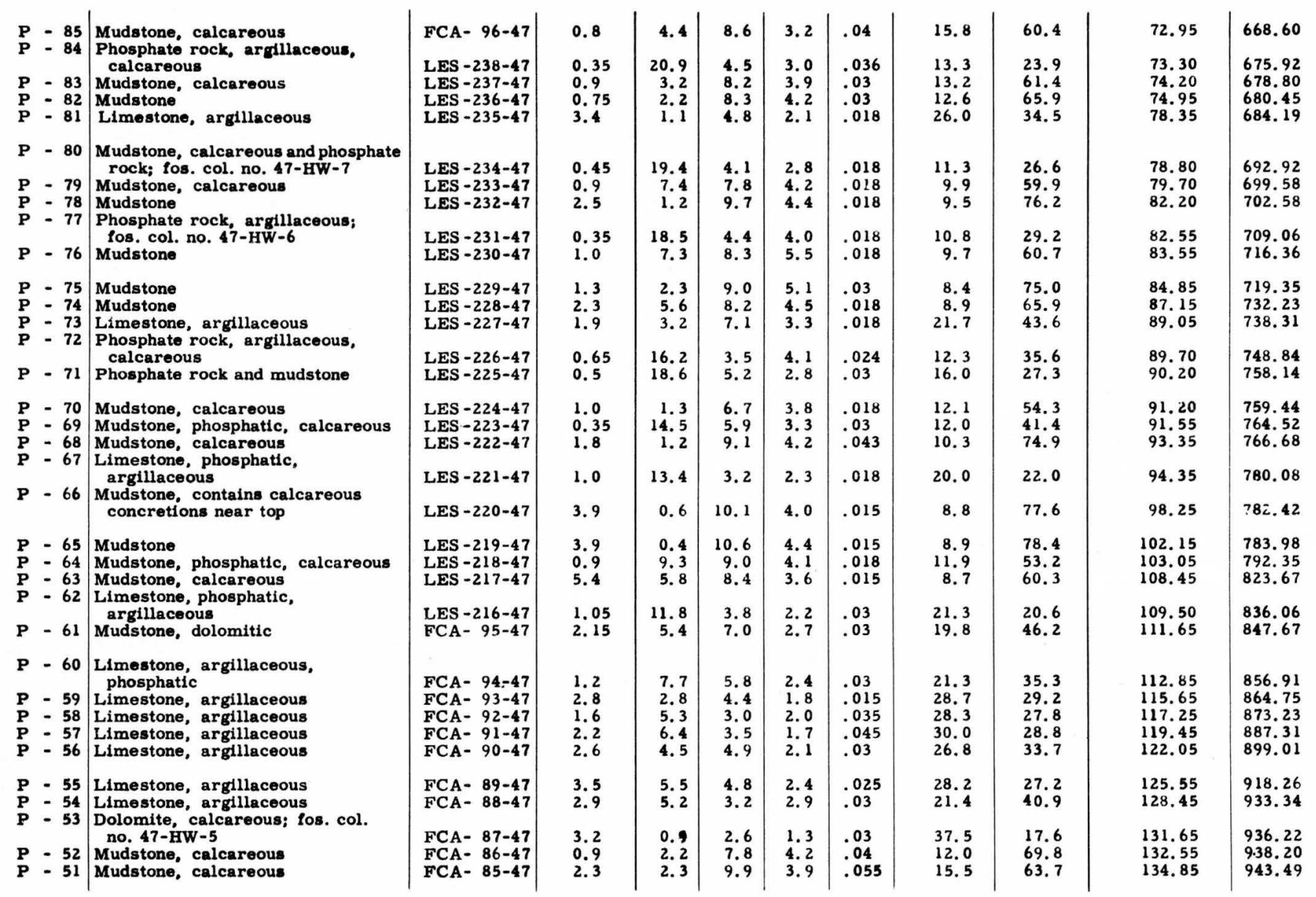

\begin{tabular}{|c|c|c|c|}
\hline .003 & .002 & .045 & \\
\hline $\begin{array}{l}.005 \\
.002 \\
.002 \\
.001\end{array}$ & $\begin{array}{l}.003 \\
.001 \\
.001 \\
.001\end{array}$ & $\begin{array}{l}.046 \\
.047 \\
.048 \\
.051\end{array}$ & \\
\hline $\begin{array}{l}.005 \\
.004 \\
.003\end{array}$ & $\begin{array}{l}.003 \\
.002 \\
.001\end{array}$ & $\begin{array}{l}.052 \\
.054 \\
.057\end{array}$ & gevers? \\
\hline $\begin{array}{l}.006 \\
.004\end{array}$ & $\begin{array}{l}.003 \\
.002\end{array}$ & $\begin{array}{r}.058 \\
.060\end{array}$ & vers \\
\hline $\begin{array}{l}.002 \\
.004 \\
.002\end{array}$ & $\begin{array}{l}.001 \\
.001 \\
.001\end{array}$ & $\begin{array}{l}.061 \\
.063 \\
.065\end{array}$ & 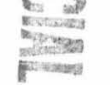 \\
\hline $\begin{array}{l}.004 \\
.006\end{array}$ & $\begin{array}{r}.003 \\
.003\end{array}$ & $\begin{array}{r}.067 \\
.069\end{array}$ & \\
\hline $\begin{array}{r}.003 \\
.004\end{array}$ & $\begin{array}{r}.001 \\
.002\end{array}$ & $\begin{array}{r}.070 \\
.070 \\
.0 *\end{array}$ & \\
\hline & $-\cdot$ & $--*$ & 43 \\
\hline .004 & -- & -- & $\operatorname{men}^{2}$ \\
\hline .002 & -- & -- & Ca \\
\hline $\begin{array}{l}.002 \\
.003 \\
.002\end{array}$ & $\begin{array}{l}-\overline{0} \\
.001 \\
.000\end{array}$ & $\begin{array}{l}\ddot{-} \\
.001 \\
.001\end{array}$ & \\
\hline $\begin{array}{l}.003 \\
.002\end{array}$ & $\begin{array}{l}.002 \\
.000\end{array}$ & $\begin{array}{r}.003 \\
.003\end{array}$ & \\
\hline $\begin{array}{l}.003 \\
.001 \\
.001 \\
.006 \\
.003\end{array}$ & $\begin{array}{l}.001 \\
.001 \\
.003 \\
.003 \\
.003\end{array}$ & $\begin{array}{l}.004 \\
.007 \\
.012 \\
.018 \\
.026\end{array}$ & \\
\hline $\begin{array}{l}.002 \\
.003\end{array}$ & $\begin{array}{r}.002 \\
.002\end{array}$ & $\begin{array}{r}.033 \\
.039\end{array}$ & \\
\hline $\begin{array}{l}.001 \\
.003 \\
.004\end{array}$ & $\begin{array}{l}.010 \\
.002 \\
.002\end{array}$ & $\begin{array}{l}.071 \\
.073 \\
.077\end{array}$ & 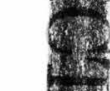 \\
\hline \multicolumn{4}{|c|}{$\begin{array}{l}\text { - Cumulative data incomplete due } \\
\text { to missing information. } \\
\text { Computations start from zero } \\
\text { after interruption. }\end{array}$} \\
\hline
\end{tabular}




\begin{tabular}{|c|c|c|c|c|c|c|c|c|c|c|c|c|c|c|c|c|}
\hline \multirow{2}{*}{\multicolumn{2}{|c|}{$\begin{array}{c}\text { Bed } \\
\text { no. }\end{array}$}} & \multirow{2}{*}{ Rock description } & \multirow{2}{*}{$\begin{array}{c}\text { Sample } \\
\text { no. }\end{array}$} & \multirow{2}{*}{$\begin{array}{c}\text { Thilckness } \\
\text { (foet) }\end{array}$} & \multicolumn{6}{|c|}{ Chomical analyses (percent) } & \multirow{2}{*}{$\begin{array}{c}\text { Cumulative } \\
\text { thickness } \\
\text { (feet) }\end{array}$} & \multirow{2}{*}{$\begin{array}{l}\text { Thickness } x \\
\text { percent } P_{2} \mathrm{O}_{5} \\
\text { (cumulative) }\end{array}$} & \multirow{2}{*}{\multicolumn{2}{|c|}{$\begin{array}{c}\text { Uranium content } \\
\text { (percent) }\end{array}$}} & \multirow{2}{*}{$\begin{array}{c}\text { Thickness x } \\
\text { percent chem. } \\
\text { (cumulative) }\end{array}$} & \\
\hline & & & & & $\mathrm{P}_{2} \mathrm{O}_{5}$ & $\mathrm{Al}_{2} \mathrm{O}_{3}$ & $\mathrm{Fe}_{2} \mathrm{O}_{3}$ & $\mathrm{v}_{2} \mathrm{O}_{5}$ & $\begin{array}{l}\text { Loss on } \\
\text { ignition }\end{array}$ & $\begin{array}{c}\text { Acid } \\
\text { insoluble }\end{array}$ & & & & & & \\
\hline P & $\begin{array}{r}-50 \\
-49 \\
-48\end{array}$ & $\begin{array}{l}\text { Mudstone, calcareous } \\
\text { Mudstone, calcareous, phosphatic }\end{array}$ & $\begin{array}{l}\text { FCA- 24-47 } \\
\text { LES }-215-47\end{array}$ & $\begin{array}{l}4.2 \\
3.2\end{array}$ & $\begin{array}{l}3.1 \\
8.9\end{array}$ & $\begin{array}{l}9.5 \\
8.0\end{array}$ & $\begin{array}{l}4.0 \\
2.9\end{array}$ & $\begin{array}{l}.01 \\
.024\end{array}$ & $\begin{array}{l}14.0 \\
13.8\end{array}$ & $\begin{array}{l}64.1 \\
45.8\end{array}$ & $\begin{array}{l}139.05 \\
142.25\end{array}$ & $\begin{array}{l}956.51 \\
984.99\end{array}$ & $\begin{array}{l}.002 \\
.003\end{array}$ & .002 & $\frac{.086}{-\infty}$ & \\
\hline & $\begin{array}{l}-48 \\
-47 \\
-46\end{array}$ & $\begin{array}{l}\text { no. 47-HW-4 } \\
\text { Mudstone, phosphatic, calcareous } \\
\text { Mudstone, phosphatic }\end{array}$ & $\begin{array}{l}\text { LES }-214-47 \\
\text { LES }-213-47 \\
\text { LES }-212-47\end{array}$ & $\begin{array}{l}1.6 \\
1.1 \\
2.0\end{array}$ & $\begin{array}{r}2.7 \\
11.8 \\
12.3\end{array}$ & $\begin{array}{l}5.2 \\
8.2 \\
7.2\end{array}$ & $\begin{array}{l}2.1 \\
3.0 \\
2.4\end{array}$ & $\begin{array}{l}.015 \\
.03 \\
.03\end{array}$ & $\begin{array}{l}27.2 \\
17.4 \\
18.8\end{array}$ & $\begin{array}{l}33.0 \\
39.3 \\
33.4\end{array}$ & $\begin{array}{l}143.85 \\
144.95 \\
146.95\end{array}$ & $\begin{array}{r}989.31 \\
1.002 .29 \\
1,026.89\end{array}$ & $\begin{array}{l}.002 \\
.003 \\
.004\end{array}$ & $\begin{array}{l}.001 \\
.002 \\
.002\end{array}$ & $\begin{array}{l}.002 \\
.004 \\
.008\end{array}$ & \\
\hline $\begin{array}{l}\mathbf{P} \\
\mathbf{P} \\
\mathbf{P} \\
\mathbf{P}\end{array}$ & $\begin{array}{l}=45 \\
=44 \\
=43 \\
=42\end{array}$ & $\begin{array}{l}\text { Limestone } \\
\text { Limestone, argillaceous } \\
\text { Mudstone, phosphatic, calcareous } \\
\text { Mudstone, phosphatic, contains }\end{array}$ & $\begin{array}{l}\text { LES }-211-47 \\
\text { LES }-210-47 \\
\text { LES }-209-47\end{array}$ & $\begin{array}{l}1.6 \\
0.8 \\
0.7\end{array}$ & $\begin{array}{r}0.3 \\
4.5 \\
11.6\end{array}$ & $\begin{array}{l}2.9 \\
5.6 \\
8.4\end{array}$ & $\begin{array}{l}1.2 \\
2.1 \\
3.0\end{array}$ & $\begin{array}{l}.015 \\
.015 \\
.03\end{array}$ & $\begin{array}{l}36.5 \\
24.5 \\
15.1\end{array}$ & $\begin{array}{l}16.7 \\
34.0 \\
44.2\end{array}$ & $\begin{array}{l}148.55 \\
149.35 \\
150.05\end{array}$ & $\begin{array}{l}1,027.37 \\
1,030.97 \\
1,039.09\end{array}$ & $\begin{array}{l}.0005 \\
.002 \\
.003\end{array}$ & $\begin{array}{l}.001 \\
.001\end{array}$ & $\begin{array}{l}.009 \\
.001\end{array}$ & 5 \\
\hline & -41 & $\begin{array}{l}\text { gypsum } \\
\text { Phosphate rock }\end{array}$ & $\begin{array}{l}\text { LES }-208-47 \\
\text { LES }-207-47\end{array}$ & $\begin{array}{l}0.45 \\
0.35\end{array}$ & $\begin{array}{l}13.7 \\
31.8\end{array}$ & $\begin{array}{l}8.5 \\
7.1\end{array}$ & $\begin{array}{l}2.6 \\
2.5\end{array}$ & $\begin{array}{l}.03 \\
.03\end{array}$ & $\begin{array}{l}17.0 \\
17.9\end{array}$ & $\begin{array}{r}37.0 \\
3.5\end{array}$ & $\begin{array}{l}150.50 \\
150.85\end{array}$ & $\begin{array}{l}1,045.26 \\
1,056.39\end{array}$ & $\begin{array}{l}.003 \\
.003\end{array}$ & $\begin{array}{l}.001 \\
.002\end{array}$ & $\begin{array}{l}.001 \\
.002 \\
\end{array}$ & \\
\hline $\begin{array}{l}\mathbf{P} \\
\mathbf{P}\end{array}$ & $\begin{array}{l}-40 \\
-39\end{array}$ & $\begin{array}{l}\text { Phosphate rock } \\
\text { Mudstone, phosphatic }\end{array}$ & $\begin{array}{l}\text { LES }-206-47 \\
\text { LES }-189-47\end{array}$ & $\begin{array}{l}1.1 \\
0.7\end{array}$ & $\begin{array}{l}31.0 \\
12.8\end{array}$ & $\begin{array}{l}7.3 \\
8.3\end{array}$ & $\begin{array}{l}2.9 \\
2.9\end{array}$ & $\begin{array}{l}.03 \\
.015\end{array}$ & $\begin{array}{l}18.4 \\
17.7\end{array}$ & $\begin{array}{r}3.8 \\
43.8\end{array}$ & $\begin{array}{l}151.95 \\
152.65\end{array}$ & $\begin{array}{l}1,090.49 \\
1,099.45\end{array}$ & $\begin{array}{l}.003 \\
.003\end{array}$ &.$\overline{001}$ & .001 & \\
\hline & & Possible shear zone, beds P-31 to & P-38: rock str & rueture lar & zely des & troyed; & stratigx & raphic & lations an & d thickness & uncertain. & & & & & \\
\hline & $\begin{array}{l}-38 \\
=37 \\
-36\end{array}$ & $\begin{array}{l}\text { Mudstone, phosphatic } \\
\text { Llmestone, dolomitic, argillaceous } \\
\text { Limestone, argillaceous, phosphatic }\end{array}$ & $\begin{array}{l}\text { LES }-188-47 \\
\text { LES }-187-47 \\
\text { LES }-186-47\end{array}$ & $\begin{array}{l}0.8 \\
0.5 \\
1.4\end{array}$ & $\begin{array}{r}13.5 \\
1.5 \\
8.1\end{array}$ & $\begin{array}{l}8.1 \\
4.9 \\
6.6\end{array}$ & $\begin{array}{l}3.1 \\
1.2 \\
2.1\end{array}$ & $\begin{array}{l}.015 \\
.01 \\
.01\end{array}$ & $\begin{array}{l}19.0 \\
35.0 \\
24.7\end{array}$ & $\begin{array}{l}39.3 \\
24.0 \\
31.8\end{array}$ & $\begin{array}{l}153.45 \\
153.95 \\
155.35\end{array}$ & $\begin{array}{l}1.110 .25 \\
1,111.00 \\
1,122.34\end{array}$ & $\begin{array}{l}.003 \\
.002 \\
.003\end{array}$ & .00 & $\begin{array}{l}--* \\
-- \\
--\end{array}$ & ments \\
\hline $\begin{array}{l}\mathbf{P} \\
\mathbf{P} \\
\mathbf{P} \\
\mathbf{P} \\
\mathbf{P}\end{array}$ & $\begin{array}{l}-33 \\
-34 \\
-33 \\
-32 \\
-31\end{array}$ & $\begin{array}{l}\text { Mudstone, phosphatic } \\
\text { Mudstone, calcareous } \\
\text { Limestone, arglllaceous, phosphatic } \\
\text { Mudstone, phosphatic, calcareous } \\
\text { Phosphate rock, calcareous }\end{array}$ & $\begin{array}{l}\text { LES }-185-47 \\
\text { LES }-184-47 \\
\text { LES }-183-47 \\
\text { LES }-182-47 \\
\text { LES }-181-47\end{array}$ & $\begin{array}{l}0.8 \\
0.6 \\
1.6 \\
1.3 \\
1.2\end{array}$ & $\begin{array}{r}12.1 \\
6.9 \\
10.1 \\
11.9 \\
18.2\end{array}$ & $\begin{array}{l}7.8 \\
7.0 \\
7.5 \\
7.2 \\
4.2\end{array}$ & $\begin{array}{l}2.9 \\
2.5 \\
2.4 \\
2.5 \\
1.6\end{array}$ & $\begin{array}{l}.015 \\
.015 \\
.010 \\
.015 \\
.040\end{array}$ & $\begin{array}{l}17.8 \\
16.4 \\
20.5 \\
18.3 \\
20.2\end{array}$ & $\begin{array}{l}40.6 \\
47.6 \\
36.0 \\
36.7 \\
18.0\end{array}$ & $\begin{array}{l}156.15 \\
156.75 \\
158.35 \\
159.65 \\
160.85\end{array}$ & $\begin{array}{l}1,132.02 \\
1,136.16 \\
1,152.32 \\
1,167.79 \\
1,189.63\end{array}$ & $\begin{array}{l}.003 \\
.003 \\
.003 \\
.004 \\
.004\end{array}$ & $\begin{array}{l}.0 \\
.001 \\
.003 \\
.002 \\
.002\end{array}$ & $\begin{array}{l}-- \\
.001 \\
.005 \\
.008 \\
.010\end{array}$ & N \\
\hline $\begin{array}{l}P \\
P \\
P \\
P \\
P\end{array}$ & $\begin{array}{l}-30 \\
=29 \\
=28 \\
=27 \\
=26\end{array}$ & $\begin{array}{l}\text { Phosphate rock, calcareous } \\
\text { Phosphate rock, argillaceous } \\
\text { Phosphate rock, calcareous } \\
\text { Limestone } \\
\text { Mudstone, phosphatic }\end{array}$ & $\begin{array}{l}\text { LES }-180-47 \\
\text { FCA- 83-47 } \\
\text { FCA- 82-47 } \\
\text { FCA- } 81-47 \\
\text { FCA } 80-47\end{array}$ & $\begin{array}{l}0.4 \\
0.4 \\
1.9 \\
2.1 \\
0.9\end{array}$ & $\begin{array}{r}15.7 \\
17.4 \\
26.0 \\
3.5 \\
12.2\end{array}$ & $\begin{array}{l}4.4 \\
5.8 \\
1.5 \\
1.5 \\
8.2\end{array}$ & $\begin{array}{l}1.7 \\
2.8 \\
1.5 \\
0.7 \\
2.9\end{array}$ & $\begin{array}{l}.050 \\
.065 \\
.03 \\
.025 \\
.03\end{array}$ & $\begin{array}{l}17.0 \\
12.7 \\
14.1 \\
28.0 \\
12.6\end{array}$ & $\begin{array}{r}27.0 \\
33.9 \\
8.8 \\
10.0 \\
43.0\end{array}$ & $\begin{array}{l}161.25 \\
161.65 \\
163.55 \\
265.65 \\
166.55\end{array}$ & $\begin{array}{l}1,195.91 \\
1,202.87 \\
1,252.27 \\
1,259.62 \\
1,270.60\end{array}$ & $\begin{array}{l}.005 \\
.005 \\
.003 \\
.001 \\
.006\end{array}$ & $\begin{array}{l}.005 \\
.002 \\
.004 \\
.001 \\
.004\end{array}$ & $\begin{array}{l}.012 \\
.013 \\
.021 \\
.023 \\
.027\end{array}$ & y \\
\hline $\begin{array}{l}\mathbf{P} \\
\mathbf{P} \\
\mathbf{P}\end{array}$ & $\begin{array}{l}-25 \\
-24 \\
-23\end{array}$ & $\begin{array}{l}\text { Limestone, dolomitic } \\
\text { Phosphate rock } \\
\text { Phosphate rock }\end{array}$ & $\begin{array}{l}\text { FCA- 79-47 } \\
\text { FCA- 78-47 } \\
\text { FCA- 77-47 }\end{array}$ & $\begin{array}{l}2.4 \\
2.5 \\
1.8\end{array}$ & $\begin{array}{r}6.4 \\
26.3 \\
29.3\end{array}$ & $\begin{array}{l}3.6 \\
2.7 \\
2.0\end{array}$ & $\begin{array}{l}1.5 \\
1.4 \\
1.2\end{array}$ & $\begin{array}{l}.03 \\
.12 \\
.26\end{array}$ & $\begin{array}{l}29.4 \\
10.0 \\
10.4\end{array}$ & $\begin{array}{l}21.0 \\
17.3 \\
12.2\end{array}$ & $\begin{array}{l}168.95 \\
171.45 \\
173.25\end{array}$ & $\begin{array}{l}1,285.96 \\
1,351.71 \\
1.404 .45\end{array}$ & $\begin{array}{l}.001 \\
.006 \\
.010\end{array}$ & $\begin{array}{l}.001 \\
.005 \\
.007\end{array}$ & $\begin{array}{l}.029 \\
.041 \\
.054\end{array}$ & 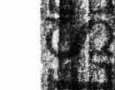 \\
\hline & $\begin{array}{l}-22 \\
-21\end{array}$ & $\begin{array}{l}\text { Phosphate rock, argillaceous, } \\
\text { calcareous } \\
\text { Phosphate rock }\end{array}$ & $\begin{array}{l}\text { FCA- } 76-47 \\
\text { FCA- } 75-47\end{array}$ & $\begin{array}{l}2.5 \\
1.4\end{array}$ & $\begin{array}{l}17.1 \\
29.6\end{array}$ & $\begin{array}{l}3.8 \\
1.8\end{array}$ & $\begin{array}{l}1.7 \\
1.0\end{array}$ & $\begin{array}{l}.13 \\
.19\end{array}$ & $\begin{array}{l}15.5 \\
10.7\end{array}$ & $\begin{array}{l}25.4 \\
11.1\end{array}$ & $\begin{array}{l}175.75 \\
177.15\end{array}$ & $\begin{array}{l}1,447.20 \\
1,488.64\end{array}$ & $\begin{array}{l}.006 \\
.009\end{array}$ & $\begin{array}{l}.004 \\
.007\end{array}$ & $\begin{array}{l}.064 \\
.074\end{array}$ & 1. \\
\hline$\vec{P}$ & $\begin{array}{r}-20 \\
-19\end{array}$ & Phosphate rock & FCA- 74-47 & 1.45 & 26.5 & 2.4 & 1.3 & .25 & 11.8 & 14.7 & 178.60 & $1,527.06$ & .009 & .008 & .085 & \\
\hline $\begin{array}{l}\mathbf{P} \\
\mathbf{P} \\
\mathbf{P}\end{array}$ & $\begin{array}{l}=18 \\
=17 \\
-16\end{array}$ & $\begin{array}{l}\text { 47-HW-3 } \\
\text { Limestone, phosphatic } \\
\text { Phosphate rock, calcareous } \\
\text { Dolomite, phosphatic }\end{array}$ & $\begin{array}{l}\text { FCA- } 73-47 \\
\text { FCA- 72-47 } \\
\text { FCA- } 71-47 \\
\text { FCA- } 70-47\end{array}$ & $\begin{array}{l}1.6 \\
2.3 \\
2.65 \\
1.6\end{array}$ & $\begin{array}{r}27.7 \\
9.3 \\
18.5 \\
8.7\end{array}$ & $\begin{array}{l}2.4 \\
3.2 \\
2.2 \\
1.2\end{array}$ & $\begin{array}{l}1.2 \\
1.4 \\
1.4 \\
0.7\end{array}$ & $\begin{array}{l}.25 \\
.1 \\
.04 \\
.03\end{array}$ & $\begin{array}{l}12.6 \\
27.2 \\
17.4 \\
33.3\end{array}$ & $\begin{array}{r}14.2 \\
20.6 \\
19.2 \\
8.0\end{array}$ & $\begin{array}{l}180.20 \\
182.50 \\
185.15 \\
: 86.75\end{array}$ & $\begin{array}{l}1,571.38 \\
1,592.77 \\
1,641.80 \\
1,655.72\end{array}$ & $\begin{array}{l}.011 \\
.003 \\
.005 \\
.001\end{array}$ & $\begin{array}{l}.009 \\
.002 \\
.004 \\
.001\end{array}$ & $\begin{array}{l}.100 \\
.104 \\
.115 \\
.117\end{array}$ & 195 \\
\hline
\end{tabular}




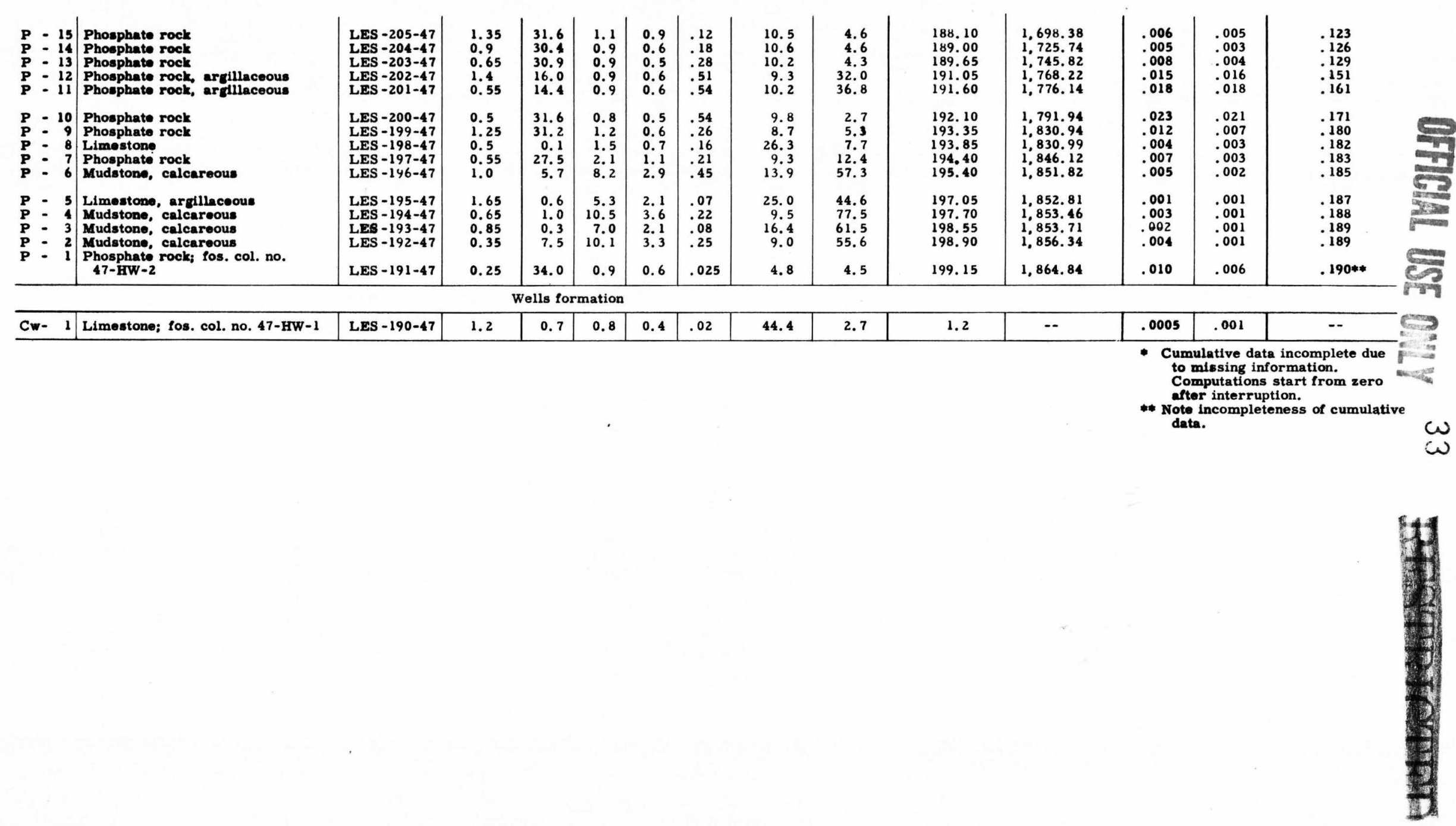


SPECTROGRAPHIC ANALYSES-CONDA, IDAHO. LOT NO. 1200.

Semi-quantitative analyses of samples of the Phosphoria formation, Conda, Idaho (see immediately preceeding pages for location of section, thickness and description of strata, and chemical analyses of samples), made by U. S. Bureau of Mines Laboratory, Albany, Oregon. In addition to the elements listed in the table below, Sb, As, Be, Bi, Cd, Co, Ga, Au, Li, Pt, Ta, Sn, and W were looked for in all samples but were not detected.

Explanation of symbols

\begin{tabular}{|c|c|c|c|c|c|c|c|c|c|c|c|c|c|c|c|c|c|c|c|c|c|c|c|}
\hline \multirow{2}{*}{\multicolumn{2}{|c|}{$\begin{array}{c}\text { Bed } \\
\text { no. }\end{array}$}} & \multirow{3}{*}{$\begin{array}{l}\text { Sample no. } \\
\text { FCA-141-47 }\end{array}$} & \multirow{3}{*}{ Al } & \multirow{3}{*}{$\mathrm{Ba}$} & \multirow{3}{*}{$\begin{array}{l}\mathbf{B} \\
\mathbf{E}\end{array}$} & \multicolumn{5}{|c|}{$\begin{array}{l}A=\text { more than } 10 \text { percent } \\
B=5-10 \text { percent } \\
C=1-5 \text { percent } \\
D=0.1-1 \text { percent }\end{array}$} & \multicolumn{6}{|c|}{$\begin{aligned} E & =0.01-0.1 \text { percent } \\
F & =0.001-0.01 \text { percent } \\
G & =\text { less than } 0.001 \text { percent } \\
N D & =\text { not detected }\end{aligned}$} & \multirow[b]{2}{*}{ Ag } & \multirow[b]{2}{*}{$\mathbf{N a}$} & \multirow[b]{2}{*}{$\mathbf{S r}$} & \multirow[b]{2}{*}{$\mathbf{T} \mathbf{i}$} & \multirow[b]{2}{*}{$\mathbf{v}$} & \multirow[b]{2}{*}{$Z_{n}$} & \multirow[b]{2}{*}{$\mathbf{Z r}$} \\
\hline & & & & & & $\mathrm{Ca}$ & $\mathrm{Cr}$ & $\mathbf{C b}$ & $\mathrm{Cu}$ & $\mathrm{Fe}$ & $\mathbf{P b}$ & $\mathbf{M g}$ & $\mathbf{M n}$ & Mo & $\mathbf{N i}$ & $\mathbf{S i}$ & & & & & & & \\
\hline $\mathbf{R}$ & -1 & & & & & C & $\mathbf{E}$ & ND & $\mathbf{G}$ & C & ND & D & $\mathbf{E}$ & $\mathbf{F}$ & $\mathbf{E}$ & $\mathbf{A}$ & ND & $\mathbf{E}$ & ND & $\mathbf{E}$ & $\mathbf{E}$ & ND & $\mathbf{F}$ \\
\hline $\begin{array}{l}\mathbf{P} \\
\mathbf{P} \\
\mathbf{P} \\
\mathbf{P}\end{array}$ & $\begin{array}{l}-142 \\
-141 \\
-140 \\
-139\end{array}$ & $\begin{array}{l}\text { FCA }-140-47 \\
\text { FCA-139-47 } \\
\text { FCA-139-47 } \\
\text { FCA-137-47 }\end{array}$ & $\begin{array}{l}\mathbf{C} \\
\mathbf{C} \\
\mathbf{C} \\
\mathbf{C}\end{array}$ & $\begin{array}{l}\text { ND } \\
\text { ND } \\
\text { ND } \\
\text { ND }\end{array}$ & $\begin{array}{l}\mathbf{F} \\
\mathbf{E} \\
\mathbf{E} \\
\mathbf{E}\end{array}$ & $\begin{array}{l}\mathbf{A} \\
\mathbf{C} \\
\mathbf{C} \\
\mathbf{C}\end{array}$ & $\begin{array}{l}\mathbf{E} \\
\mathbf{E} \\
\mathbf{E} \\
\mathbf{E}\end{array}$ & $\begin{array}{l}\text { ND } \\
\text { ND } \\
\text { ND } \\
\text { ND }\end{array}$ & $\begin{array}{l}\mathbf{G} \\
\mathbf{G} \\
\mathbf{G} \\
\mathbf{G}\end{array}$ & $\begin{array}{l}\mathbf{D} \\
\mathbf{C} \\
\mathbf{C} \\
\mathbf{C}\end{array}$ & $\begin{array}{l}\text { ND } \\
\text { ND } \\
\text { ND } \\
\text { ND }\end{array}$ & $\begin{array}{l}\mathbf{D} \\
\mathbf{D} \\
\mathbf{D} \\
\mathbf{D}\end{array}$ & $\begin{array}{l}\mathbf{E} \\
\mathbf{E} \\
\mathbf{E} \\
\mathbf{E}\end{array}$ & $\begin{array}{l}\mathbf{F} \\
\mathbf{F} \\
\mathbf{F} \\
\mathbf{F}\end{array}$ & $\begin{array}{l}\mathbf{E} \\
\mathbf{E} \\
\mathbf{E} \\
\mathbf{E}\end{array}$ & $\begin{array}{l}\mathbf{C} \\
\mathbf{A} \\
\mathbf{A} \\
\mathbf{A}\end{array}$ & $\begin{array}{c}\text { ND } \\
\text { G } \\
\text { ND } \\
\text { ND }\end{array}$ & $\begin{array}{l}\mathbf{E} \\
\mathbf{D} \\
\mathbf{D} \\
\mathbf{E}\end{array}$ & $\begin{array}{c}\mathbf{E} \\
\mathbf{E} \\
\mathbf{N D} \\
\mathbf{N D}\end{array}$ & $\begin{array}{l}\mathbf{E} \\
\mathbf{E} \\
\mathbf{E} \\
\mathbf{E}\end{array}$ & $\begin{array}{l}\mathbf{D} \\
\mathbf{E} \\
\mathbf{D} \\
\mathbf{E}\end{array}$ & $\begin{array}{c}\mathbf{E} \\
\mathbf{D} \\
\mathbf{N D} \\
\mathbf{E}\end{array}$ & $\begin{array}{l}\mathbf{F} \\
\mathbf{E} \\
\mathbf{F} \\
\mathbf{F}\end{array}$ \\
\hline & & Beds P-138 & ugh & -136 & t & $1=$ & & & & & & & & & & & & & & & & & \\
\hline $\mathbf{P}$ & -135 & FCA-136-47 & C & ND & $\mathbf{F}$ & $\mathbf{A}$ & $\mathbf{E}$ & ND & $\mathbf{G}$ & D & ND & $\mathbf{D}$ & $\mathbf{E}$ & $\mathbf{F}$ & $\mathbf{E}$ & C & G & D & $\mathbf{E}$ & $\mathbf{E}$ & D & $\mathbf{E}$ & $\mathbf{F}$ \\
\hline $\begin{array}{l}\mathbf{P} \\
\mathbf{P} \\
\mathbf{P} \\
\mathbf{P} \\
\mathbf{P}\end{array}$ & $\begin{array}{l}-134 \\
-133 \\
-132 \\
-131 \\
-130\end{array}$ & $\begin{array}{l}\text { FCA-135-47 } \\
\text { FCA-134-47 } \\
\text { FCA-133-47 } \\
\text { FCA-132-47 } \\
\text { FCA-131-47 }\end{array}$ & $\begin{array}{l}\text { C } \\
\text { D } \\
\text { D } \\
\text { C } \\
\text { D }\end{array}$ & $\begin{array}{c}\text { ND } \\
\text { ND } \\
\text { ND } \\
\mathbf{E} \\
\mathbf{E}\end{array}$ & $\begin{array}{l}\mathbf{E} \\
\mathbf{F} \\
\mathbf{F} \\
\mathbf{F} \\
\mathbf{F}\end{array}$ & $\begin{array}{l}\mathbf{C} \\
\mathbf{A} \\
\mathbf{A} \\
\mathbf{A} \\
\mathbf{A}\end{array}$ & $\begin{array}{l}\mathbf{E} \\
\mathbf{E} \\
\mathbf{E} \\
\mathbf{D} \\
\mathbf{E}\end{array}$ & $\begin{array}{l}\text { ND } \\
\text { ND } \\
\text { ND } \\
\text { ND } \\
\text { ND }\end{array}$ & $\begin{array}{l}\mathbf{G} \\
\mathbf{G} \\
\mathbf{G} \\
\mathbf{G} \\
\mathbf{G}\end{array}$ & $\begin{array}{l}\text { C } \\
\text { D } \\
\text { D } \\
\text { C } \\
\text { C }\end{array}$ & $\begin{array}{l}\text { ND } \\
\text { ND } \\
\text { ND } \\
\text { ND } \\
\text { ND }\end{array}$ & $\begin{array}{l}\mathbf{D} \\
\mathbf{D} \\
\mathbf{D} \\
\mathbf{D} \\
\mathbf{D}\end{array}$ & $\begin{array}{l}\mathbf{E} \\
\mathbf{F} \\
\mathbf{F} \\
\mathbf{F} \\
\mathbf{F}\end{array}$ & $\begin{array}{l}\mathbf{E} \\
\mathbf{F} \\
\mathbf{F} \\
\mathbf{E} \\
\mathbf{F}\end{array}$ & $\begin{array}{l}\mathbf{E} \\
\mathbf{F} \\
\mathbf{F} \\
\mathbf{E} \\
\mathbf{F}\end{array}$ & $\begin{array}{l}\text { A } \\
\mathbf{D} \\
\mathbf{D} \\
\mathbf{B} \\
\mathbf{C}\end{array}$ & $\begin{array}{l}\mathbf{G} \\
\mathbf{G} \\
\mathbf{G} \\
\mathbf{G} \\
\mathbf{G}\end{array}$ & $\begin{array}{l}\mathbf{D} \\
\mathbf{E} \\
\mathbf{E} \\
\mathbf{E} \\
\mathbf{E}\end{array}$ & $\begin{array}{l}\mathbf{E} \\
\mathbf{E} \\
\mathbf{E} \\
\mathbf{E} \\
\mathbf{E}\end{array}$ & $\begin{array}{l}\mathbf{D} \\
\mathbf{F} \\
\mathbf{F} \\
\mathbf{E} \\
\mathbf{E}\end{array}$ & $\begin{array}{l}\mathbf{D} \\
\mathbf{E} \\
\mathbf{E} \\
\mathbf{D} \\
\mathbf{E}\end{array}$ & $\begin{array}{l}\mathbf{D} \\
\mathbf{E} \\
\mathbf{E} \\
\mathbf{D} \\
\mathbf{E}\end{array}$ & $\begin{array}{l}\mathbf{F} \\
\mathbf{F} \\
\mathbf{F} \\
\mathbf{F} \\
\mathbf{F}\end{array}$ \\
\hline $\begin{array}{l}\mathbf{P} \\
\mathbf{P} \\
\mathbf{P} \\
\mathbf{P} \\
\mathbf{P}\end{array}$ & $\begin{array}{l}-129 \\
-128 \\
-127 \\
-126 \\
-125\end{array}$ & $\begin{array}{l}\text { FCA-130-47 } \\
\text { FCA-129-47 } \\
\text { FCA-128-47 } \\
\text { FCA-127-47 } \\
\text { FCA-126-47 }\end{array}$ & $\begin{array}{l}\mathbf{C} \\
\mathbf{C} \\
\mathbf{C} \\
\mathbf{C} \\
\mathbf{C}\end{array}$ & $\begin{array}{l}\mathbf{E} \\
\mathbf{E} \\
\mathbf{E} \\
\mathbf{E} \\
\mathbf{E}\end{array}$ & $\begin{array}{l}\mathbf{F} \\
\mathbf{F} \\
\mathbf{F} \\
\mathbf{F} \\
\mathbf{F}\end{array}$ & $\begin{array}{l}\text { A } \\
\text { A } \\
\text { A } \\
\text { A } \\
\text { A }\end{array}$ & $\begin{array}{l}\mathbf{E} \\
\mathbf{E} \\
\mathbf{E} \\
\mathbf{E} \\
\mathbf{E}\end{array}$ & $\begin{array}{l}\text { ND } \\
\text { ND } \\
\text { ND } \\
\text { ND } \\
\text { ND }\end{array}$ & $\begin{array}{l}\mathbf{G} \\
\mathbf{G} \\
\mathbf{G} \\
\mathbf{G} \\
\mathbf{G}\end{array}$ & $\begin{array}{l}\mathbf{C} \\
\mathbf{C} \\
\mathbf{C} \\
\mathbf{C} \\
\mathbf{C}\end{array}$ & $\begin{array}{l}\text { ND } \\
\text { ND } \\
\text { ND } \\
\text { ND } \\
\text { ND }\end{array}$ & $\begin{array}{l}\text { D } \\
\text { D } \\
\text { D } \\
\text { D } \\
\text { D }\end{array}$ & $\begin{array}{l}\mathbf{F} \\
\mathbf{F} \\
\mathbf{F} \\
\mathbf{F} \\
\mathbf{F}\end{array}$ & $\begin{array}{l}\mathbf{F} \\
\mathbf{F} \\
\mathbf{F} \\
\mathbf{F} \\
\mathbf{F}\end{array}$ & $\begin{array}{l}\mathbf{E} \\
\mathbf{F} \\
\mathbf{E} \\
\mathbf{E} \\
\mathbf{E}\end{array}$ & $\begin{array}{l}\text { B } \\
\text { C } \\
\text { C } \\
\text { C } \\
\text { C }\end{array}$ & $\begin{array}{l}\mathbf{G} \\
\mathbf{G} \\
\mathbf{G} \\
\mathbf{G} \\
\mathbf{G}\end{array}$ & $\begin{array}{l}\mathbf{E} \\
\mathbf{E} \\
\mathbf{E} \\
\mathbf{E} \\
\mathbf{E}\end{array}$ & $\begin{array}{l}\mathbf{E} \\
\mathbf{F} \\
\mathbf{E} \\
\mathbf{E} \\
\mathbf{E}\end{array}$ & $\begin{array}{l}\mathbf{E} \\
\mathbf{E} \\
\mathbf{E} \\
\mathbf{E} \\
\mathbf{E}\end{array}$ & $\begin{array}{l}\mathbf{E} \\
\mathbf{E} \\
\mathbf{D} \\
\mathbf{D} \\
\mathbf{D}\end{array}$ & $\begin{array}{c}\mathbf{E} \\
\mathbf{N D} \\
\mathbf{E} \\
\mathbf{E} \\
\mathbf{E}\end{array}$ & $\begin{array}{l}\mathbf{F} \\
\mathbf{F} \\
\mathbf{F} \\
\mathbf{F} \\
\mathbf{F}\end{array}$ \\
\hline $\begin{array}{l}\mathbf{P} \\
\mathbf{P} \\
\mathbf{P} \\
\mathbf{P} \\
\mathbf{P}\end{array}$ & $\begin{array}{l}-124 \\
-123 \\
-122 \\
-121 \\
-120\end{array}$ & $\begin{array}{l}\text { FCA-125-47 } \\
\text { FCA-124-47 } \\
\text { FCA-123-47 } \\
\text { FCA-122-47 } \\
\text { FCA-121-47 }\end{array}$ & $\begin{array}{l}\mathbf{C} \\
\mathbf{C} \\
\mathbf{C} \\
\mathbf{C} \\
\mathbf{D}\end{array}$ & $\begin{array}{l}\mathbf{E} \\
\mathbf{E} \\
\mathbf{E} \\
\mathbf{E} \\
\mathbf{E}\end{array}$ & $\begin{array}{l}\mathbf{F} \\
\mathbf{F} \\
\mathbf{E} \\
\mathbf{F} \\
\mathbf{F}\end{array}$ & $\begin{array}{l}\mathbf{A} \\
\mathbf{A} \\
\mathbf{A} \\
\mathbf{A} \\
\mathbf{A}\end{array}$ & $\begin{array}{l}\mathbf{E} \\
\mathbf{E} \\
\mathbf{E} \\
\mathbf{E} \\
\mathbf{E}\end{array}$ & $\begin{array}{c}\text { ND } \\
\text { ND } \\
\mathbf{E} \\
\mathbf{E} \\
\mathbf{N D}\end{array}$ & $\begin{array}{l}\mathbf{G} \\
\mathbf{G} \\
\mathbf{G} \\
\mathbf{G} \\
\mathbf{G}\end{array}$ & $\begin{array}{l}\text { D } \\
\text { D } \\
\text { C } \\
\text { D } \\
\text { D }\end{array}$ & $\begin{array}{l}\text { ND } \\
\text { ND } \\
\text { ND } \\
\text { ND } \\
\text { ND }\end{array}$ & $\begin{array}{l}\text { C } \\
\mathbf{D} \\
\mathbf{D} \\
\mathbf{D} \\
\mathbf{C}\end{array}$ & $\begin{array}{l}\mathbf{F} \\
\mathbf{F} \\
\mathbf{F} \\
\mathbf{F} \\
\mathbf{F}\end{array}$ & $\begin{array}{l}\mathbf{F} \\
\mathbf{F} \\
\mathbf{E} \\
\mathbf{E} \\
\mathbf{F}\end{array}$ & $\begin{array}{l}\mathbf{F} \\
\mathbf{E} \\
\mathbf{E} \\
\mathbf{E} \\
\mathbf{F}\end{array}$ & $\begin{array}{l}\text { C } \\
\text { C } \\
\text { A } \\
\text { B } \\
\text { C }\end{array}$ & $\begin{array}{l}\mathbf{G} \\
\mathbf{G} \\
\mathbf{G} \\
\mathbf{G} \\
\mathbf{G}\end{array}$ & $\begin{array}{l}\mathbf{E} \\
\mathbf{E} \\
\mathbf{E} \\
\mathbf{E} \\
\mathbf{E}\end{array}$ & $\begin{array}{l}\mathbf{F} \\
\mathbf{E} \\
\mathbf{E} \\
\mathbf{E} \\
\mathbf{E}\end{array}$ & $\begin{array}{l}\mathbf{E} \\
\mathbf{E} \\
\mathbf{E} \\
\mathbf{E} \\
\mathbf{E}\end{array}$ & $\begin{array}{l}\mathbf{E} \\
\mathbf{E} \\
\mathbf{D} \\
\mathbf{D} \\
\mathbf{E}\end{array}$ & $\begin{array}{c}\mathbf{N D} \\
\mathbf{E} \\
\mathbf{D} \\
\mathbf{E} \\
\mathbf{N D}\end{array}$ & $\begin{array}{l}\mathbf{F} \\
\mathbf{F} \\
\mathbf{E} \\
\mathbf{F} \\
\mathbf{F}\end{array}$ \\
\hline $\begin{array}{l}\mathbf{P} \\
\mathbf{P} \\
\mathbf{P} \\
\mathbf{P} \\
\mathbf{P}\end{array}$ & $\begin{array}{l}-119 \\
-118 \\
-117 \\
-116 \\
-115\end{array}$ & $\begin{array}{l}\text { FCA-120-47 } \\
\text { FCA-119-47 } \\
\text { FCA-118-47 } \\
\text { FCA-117-47 } \\
\text { FCA-116-47 }\end{array}$ & $\begin{array}{l}\mathbf{C} \\
\mathbf{C} \\
\mathbf{C} \\
\mathbf{C} \\
\mathbf{C}\end{array}$ & $\begin{array}{l}\mathbf{E} \\
\mathbf{E} \\
\mathbf{E} \\
\mathbf{E} \\
\mathbf{E}\end{array}$ & $\begin{array}{l}\mathbf{F} \\
\mathbf{E} \\
\mathbf{E} \\
\mathbf{E} \\
\mathbf{E}\end{array}$ & $\begin{array}{l}\mathbf{A} \\
\mathbf{B} \\
\mathbf{C} \\
\mathbf{C} \\
\mathbf{C}\end{array}$ & $\begin{array}{l}\mathbf{E} \\
\mathbf{E} \\
\mathbf{E} \\
\mathbf{E} \\
\mathbf{E}\end{array}$ & $\begin{array}{l}\mathbf{E} \\
\mathbf{E} \\
\mathbf{E} \\
\mathbf{E} \\
\mathbf{E}\end{array}$ & $\begin{array}{l}\mathbf{G} \\
\mathbf{G} \\
\mathbf{G} \\
\mathbf{G} \\
\mathbf{G}\end{array}$ & $\begin{array}{l}\mathbf{C} \\
\mathbf{C} \\
\mathbf{C} \\
\mathbf{C} \\
\mathbf{C}\end{array}$ & $\begin{array}{l}\text { ND } \\
\text { ND } \\
\text { ND } \\
\text { ND } \\
\text { ND }\end{array}$ & $\begin{array}{l}\text { C } \\
\text { D } \\
\text { D } \\
\text { D } \\
\text { D }\end{array}$ & $\begin{array}{l}\mathbf{E} \\
\mathbf{F} \\
\mathbf{F} \\
\mathbf{F} \\
\mathbf{E}\end{array}$ & $\begin{array}{l}\mathbf{E} \\
\mathbf{E} \\
\mathbf{E} \\
\mathbf{E} \\
\mathbf{E}\end{array}$ & $\begin{array}{l}\mathbf{E} \\
\mathbf{E} \\
\mathbf{E} \\
\mathbf{E} \\
\mathbf{E}\end{array}$ & $\begin{array}{l}\mathbf{A} \\
\mathbf{A} \\
\mathbf{A} \\
\mathbf{A} \\
\mathbf{A}\end{array}$ & $\begin{array}{l}\mathbf{G} \\
\mathbf{G} \\
\mathbf{G} \\
\mathbf{G} \\
\mathbf{G}\end{array}$ & $\begin{array}{l}\mathbf{D} \\
\mathbf{D} \\
\mathbf{D} \\
\mathbf{D} \\
\mathbf{D}\end{array}$ & $\begin{array}{l}\mathbf{E} \\
\mathbf{F} \\
\mathbf{F} \\
\mathbf{F} \\
\mathbf{F}\end{array}$ & $\begin{array}{l}\mathbf{E} \\
\mathbf{E} \\
\mathbf{E} \\
\mathbf{E} \\
\mathbf{E}\end{array}$ & $\begin{array}{l}\text { D } \\
\text { D } \\
\text { D } \\
\text { D } \\
\text { D }\end{array}$ & $\begin{array}{l}\mathbf{E} \\
\mathbf{E} \\
\mathbf{D} \\
\mathbf{E} \\
\mathbf{E}\end{array}$ & $\begin{array}{l}\mathbf{F} \\
\mathbf{F} \\
\mathbf{F} \\
\mathbf{F} \\
\mathbf{E}\end{array}$ \\
\hline
\end{tabular}




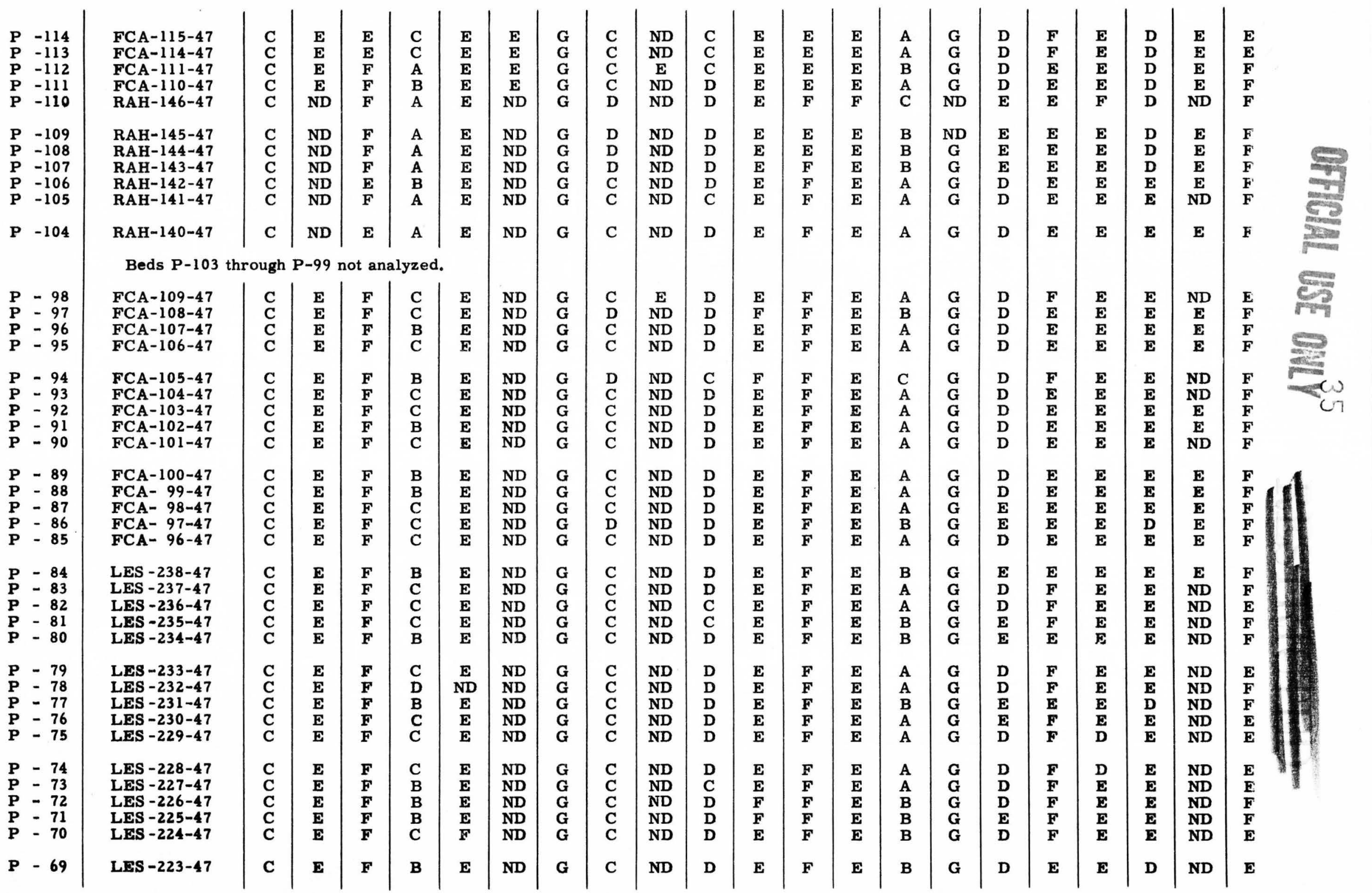




\begin{tabular}{|c|c|c|c|c|c|c|c|c|c|c|c|c|c|c|c|c|c|c|c|c|c|c|}
\hline $\begin{array}{c}\text { Bed } \\
\text { no. }\end{array}$ & Sample no. & Al & $\mathbf{B a}$ & B & $\mathrm{Ca}$ & $\mathrm{Cr}$ & $\mathrm{Cb}$ & $\mathrm{Cu}$ & $\mathrm{Fe}$ & $\mathbf{P b}$ & Mg & Mn & Mo & $\mathrm{Ni}$ & $\mathbf{S i}$ & Ag & $\mathrm{Na}$ & $\mathbf{S r}$ & $\mathbf{T i}$ & $\mathbf{v}$ & Zn & $\mathrm{Zr}$ \\
\hline $\begin{array}{l}P=68 \\
P=67 \\
P=66 \\
P=65 \\
P=64\end{array}$ & $\begin{array}{l}\text { LES }-222-47 \\
\text { LES }-221-47 \\
\text { LES }-220-47 \\
\text { LES }-219-47 \\
\text { LES }-218-47\end{array}$ & $\begin{array}{l}\mathbf{C} \\
\mathbf{C} \\
\mathbf{C} \\
\mathbf{C} \\
\mathbf{C}\end{array}$ & $\begin{array}{l}\mathbf{E} \\
\mathbf{E} \\
\mathbf{E} \\
\mathbf{E} \\
\mathbf{E}\end{array}$ & $\begin{array}{l}\mathbf{F} \\
\mathbf{F} \\
\mathbf{F} \\
\mathbf{F} \\
\mathbf{F}\end{array}$ & $\begin{array}{l}\text { C } \\
\text { C } \\
\text { D } \\
\text { D } \\
\text { C }\end{array}$ & $\begin{array}{l}\mathbf{E} \\
\mathbf{E} \\
\mathbf{F} \\
\mathbf{F} \\
\mathbf{E}\end{array}$ & $\begin{array}{l}\text { ND } \\
\text { ND } \\
\text { ND } \\
\text { ND } \\
\text { ND }\end{array}$ & $\begin{array}{l}\mathbf{G} \\
\mathbf{G} \\
\mathbf{G} \\
\mathbf{G} \\
\mathbf{G}\end{array}$ & $\begin{array}{l}\mathbf{C} \\
\mathbf{D} \\
\mathbf{C} \\
\mathbf{C} \\
\mathbf{D}\end{array}$ & $\begin{array}{l}\text { ND } \\
\text { ND } \\
\text { ND } \\
\text { ND } \\
\text { ND }\end{array}$ & $\begin{array}{l}\text { D } \\
\text { D } \\
\text { D } \\
\text { D } \\
\text { D }\end{array}$ & $\begin{array}{l}\mathbf{E} \\
\mathbf{E} \\
\mathbf{E} \\
\mathbf{E} \\
\mathbf{F}\end{array}$ & $\begin{array}{l}\mathbf{F} \\
\mathbf{F} \\
\mathbf{F} \\
\mathbf{F} \\
\mathbf{F}\end{array}$ & $\begin{array}{l}\mathbf{E} \\
\mathbf{E} \\
\mathbf{E} \\
\mathbf{E} \\
\mathbf{E}\end{array}$ & $\begin{array}{l}\text { A } \\
\text { C } \\
\text { A } \\
\text { A } \\
\text { B }\end{array}$ & $\begin{array}{l}\mathrm{G} \\
\mathrm{ND} \\
\mathrm{ND} \\
\mathrm{ND} \\
\mathrm{ND}\end{array}$ & $\begin{array}{l}\text { D } \\
\text { D } \\
\text { D } \\
\text { D } \\
\text { D }\end{array}$ & $\begin{array}{l}\mathbf{F} \\
\mathbf{F} \\
\text { ND } \\
\text { ND } \\
\text { ND }\end{array}$ & $\begin{array}{l}\mathbf{D} \\
\mathbf{E} \\
\mathbf{E} \\
\mathbf{E} \\
\mathbf{E}\end{array}$ & $\begin{array}{l}\mathbf{E} \\
\mathbf{E} \\
\mathbf{E} \\
\mathbf{E} \\
\mathbf{E}\end{array}$ & $\begin{array}{l}\text { ND } \\
\text { ND } \\
\text { ND } \\
\text { ND } \\
\text { ND }\end{array}$ & $\begin{array}{l}\mathbf{E} \\
\mathbf{F} \\
\mathbf{E} \\
\mathbf{E} \\
\mathbf{F}\end{array}$ \\
\hline $\begin{array}{l}P=63 \\
P=62 \\
P=61 \\
P=60 \\
P=59\end{array}$ & $\begin{array}{l}\text { LES }-217-47 \\
\text { LES }-216-47 \\
\text { FCA- } 95-47 \\
\text { FCA- } 94-47 \\
\text { FCA- } 93-47\end{array}$ & $\begin{array}{l}\mathbf{C} \\
\mathbf{C} \\
\mathbf{C} \\
\mathbf{C} \\
\mathbf{C}\end{array}$ & $\begin{array}{l}\mathbf{E} \\
\mathbf{E} \\
\mathbf{E} \\
\mathbf{E} \\
\mathbf{E}\end{array}$ & $\begin{array}{l}\mathbf{F} \\
\mathbf{F} \\
\mathbf{F} \\
\mathbf{F} \\
\mathbf{F}\end{array}$ & $\begin{array}{l}\mathbf{C} \\
\mathbf{B} \\
\mathbf{C} \\
\mathbf{B} \\
\mathbf{B}\end{array}$ & $\begin{array}{l}E \\
E \\
E \\
E \\
E\end{array}$ & $\begin{array}{l}\text { ND } \\
\text { ND } \\
\text { ND } \\
\text { ND } \\
\text { ND }\end{array}$ & $\begin{array}{l}\mathbf{G} \\
\mathbf{G} \\
\mathbf{G} \\
\mathbf{G} \\
\mathbf{G}\end{array}$ & $\begin{array}{l}\text { C } \\
\text { D } \\
C \\
C \\
\text { D }\end{array}$ & $\begin{array}{l}\text { ND } \\
\text { ND } \\
\text { ND } \\
\text { ND } \\
\text { ND }\end{array}$ & $\begin{array}{l}\mathrm{D} \\
\mathrm{D} \\
\mathrm{D} \\
\mathrm{D} \\
\mathrm{D}\end{array}$ & $\begin{array}{l}\mathbf{E} \\
\mathbf{E} \\
\mathbf{E} \\
\mathbf{E} \\
\mathbf{E}\end{array}$ & $\begin{array}{l}\mathbf{F} \\
\mathbf{F} \\
\mathbf{F} \\
\mathbf{F} \\
\mathbf{F}\end{array}$ & $\begin{array}{l}\mathbf{E} \\
\mathbf{E} \\
\mathbf{E} \\
\mathbf{E} \\
\mathbf{E}\end{array}$ & $\begin{array}{l}\text { A } \\
\text { C } \\
\text { A } \\
\text { A } \\
\text { B }\end{array}$ & $\begin{array}{l}\mathbf{G} \\
\mathbf{G} \\
\mathbf{G} \\
\mathbf{G} \\
\mathbf{G}\end{array}$ & $\begin{array}{l}\mathbf{D} \\
\mathbf{D} \\
\mathbf{D} \\
\mathbf{D} \\
\mathbf{D}\end{array}$ & $\begin{array}{l}\mathbf{F} \\
\mathbf{F} \\
\mathbf{E} \\
\mathbf{E} \\
\mathbf{E}\end{array}$ & $\begin{array}{l}\mathbf{E} \\
\mathbf{E} \\
\mathbf{E} \\
\mathbf{E} \\
\mathbf{E}\end{array}$ & $\begin{array}{l}\mathbf{E} \\
\mathbf{E} \\
\mathbf{E} \\
\mathbf{E} \\
\mathbf{E}\end{array}$ & $\begin{array}{l}\mathbf{E} \\
\mathbf{E} \\
\mathbf{E} \\
\mathbf{E} \\
\mathbf{N D}\end{array}$ & $\begin{array}{l}E \\
F \\
F \\
F \\
F\end{array}$ \\
\hline $\begin{array}{l}P=58 \\
P=57 \\
P=56 \\
P=55 \\
P=54\end{array}$ & $\begin{array}{l}\text { FCA- } 92-47 \\
\text { FCA- } 91-47 \\
\text { FCA- } 90-47 \\
\text { FCA- } 89-47 \\
\text { FCA- } 88-47\end{array}$ & $\begin{array}{l}\mathbf{C} \\
\mathbf{C} \\
\mathbf{C} \\
\mathbf{C} \\
\mathbf{C}\end{array}$ & $\begin{array}{l}\mathbf{E} \\
\mathbf{E} \\
\mathbf{E} \\
\mathbf{E} \\
\mathbf{E}\end{array}$ & $\begin{array}{l}\mathbf{F} \\
\mathbf{F} \\
\mathbf{F} \\
\mathbf{F} \\
\mathbf{F}\end{array}$ & $\begin{array}{l}\mathbf{C} \\
\mathbf{C} \\
\mathbf{C} \\
\mathbf{C} \\
\mathbf{C}\end{array}$ & $\begin{array}{l}\mathbf{D} \\
\mathbf{D} \\
\mathbf{E} \\
\mathbf{E} \\
\mathbf{E}\end{array}$ & $\begin{array}{l}\text { ND } \\
\text { ND } \\
\text { ND } \\
\text { ND } \\
\text { ND }\end{array}$ & $\begin{array}{l}\mathbf{G} \\
\mathbf{G} \\
\mathbf{G} \\
\mathbf{G} \\
\mathbf{G}\end{array}$ & $\begin{array}{l}\mathrm{C} \\
\mathrm{C} \\
\mathrm{C} \\
\mathrm{C} \\
\mathrm{C}\end{array}$ & $\begin{array}{l}\text { ND } \\
\text { ND } \\
\text { ND } \\
\text { ND } \\
\text { ND }\end{array}$ & $\begin{array}{l}\mathrm{D} \\
\mathrm{D} \\
\mathrm{D} \\
\mathrm{D} \\
\mathrm{D}\end{array}$ & $\begin{array}{l}\mathbf{E} \\
\mathbf{E} \\
\mathbf{E} \\
\mathbf{E} \\
\mathbf{E}\end{array}$ & $\begin{array}{l}\mathbf{F} \\
\mathbf{E} \\
\mathbf{F} \\
\mathbf{F} \\
\mathbf{F}\end{array}$ & $\begin{array}{l}\mathbf{E} \\
\mathbf{E} \\
\mathbf{E} \\
\mathbf{E} \\
\mathbf{E}\end{array}$ & $\begin{array}{l}\text { A } \\
\text { A } \\
\text { B } \\
\text { C } \\
\text { A }\end{array}$ & $\begin{array}{l}\mathbf{G} \\
\mathbf{G} \\
\mathbf{G} \\
\mathbf{G} \\
\mathbf{G}\end{array}$ & $\begin{array}{l}\mathbf{E} \\
\mathbf{E} \\
\mathbf{D} \\
\mathbf{E} \\
\mathbf{D}\end{array}$ & $\begin{array}{l}\mathbf{E} \\
\mathbf{E} \\
\mathbf{E} \\
\mathbf{E} \\
\mathbf{E}\end{array}$ & $\begin{array}{l}\mathbf{E} \\
\mathbf{E} \\
\mathbf{E} \\
\mathbf{E} \\
\mathbf{E}\end{array}$ & $\begin{array}{l}\mathbf{E} \\
\mathbf{D} \\
\mathbf{E} \\
\mathbf{E} \\
\mathbf{E}\end{array}$ & $\begin{array}{c}\mathbf{E} \\
\mathbf{E} \\
\mathbf{N D} \\
\mathbf{E} \\
\mathbf{N D}\end{array}$ & $\begin{array}{l}\mathbf{F} \\
\mathbf{F} \\
\mathbf{F} \\
\mathbf{F} \\
\mathbf{F}\end{array}$ \\
\hline $\begin{array}{l}P=53 \\
P=52 \\
P=51 \\
P=50 \\
P=49\end{array}$ & $\begin{array}{l}\text { FCA- } 87-47 \\
\text { FCA- } 86-47 \\
\text { FCA- 85-47 } \\
\text { FCA- 84-47 } \\
\text { LES }-215-47\end{array}$ & $\begin{array}{l}\mathbf{C} \\
\mathbf{C} \\
\mathbf{C} \\
\mathbf{C} \\
\mathbf{C}\end{array}$ & $\begin{array}{l}\mathbf{E} \\
\mathbf{E} \\
\mathbf{E} \\
\mathbf{E} \\
\mathbf{E}\end{array}$ & $\begin{array}{l}\mathbf{F} \\
\mathbf{F} \\
\mathbf{F} \\
\mathbf{F} \\
\mathbf{F}\end{array}$ & $\begin{array}{l}\text { B } \\
\mathbf{C} \\
\mathbf{C} \\
\mathbf{C} \\
\mathbf{C}\end{array}$ & $\begin{array}{l}\mathbf{E} \\
\mathbf{E} \\
\mathbf{E} \\
\mathbf{E} \\
\mathbf{E}\end{array}$ & $\begin{array}{l}\text { ND } \\
\text { ND } \\
\text { ND } \\
\text { ND } \\
\text { ND }\end{array}$ & $\begin{array}{l}\mathbf{G} \\
\mathbf{G} \\
\mathbf{G} \\
\mathbf{G} \\
\mathbf{G}\end{array}$ & $\begin{array}{l}\mathrm{D} \\
\mathrm{C} \\
\mathrm{C} \\
\mathrm{D} \\
\mathrm{C}\end{array}$ & $\begin{array}{l}\text { ND } \\
\text { ND } \\
\text { ND } \\
\text { ND } \\
\text { ND }\end{array}$ & $\begin{array}{l}\text { C } \\
\text { D } \\
\text { D } \\
\text { D } \\
\text { D }\end{array}$ & $\begin{array}{l}\mathbf{E} \\
\mathbf{E} \\
\mathbf{E} \\
\mathbf{E} \\
\mathbf{E}\end{array}$ & $\begin{array}{l}\mathbf{F} \\
\mathbf{F} \\
\mathbf{F} \\
\mathbf{F} \\
\mathbf{F}\end{array}$ & $\begin{array}{l}\mathbf{E} \\
\mathbf{E} \\
\mathbf{E} \\
\mathbf{E} \\
\mathbf{E}\end{array}$ & $\begin{array}{l}\text { C } \\
\text { A } \\
\text { A } \\
\text { A } \\
\text { A }\end{array}$ & $\begin{array}{l}\mathbf{G} \\
\mathbf{G} \\
\mathbf{G} \\
\mathbf{G} \\
\mathbf{G}\end{array}$ & $\begin{array}{l}\mathbf{E} \\
\mathbf{D} \\
\mathbf{D} \\
\mathbf{E} \\
\mathbf{D}\end{array}$ & $\begin{array}{l}F \\
F \\
F \\
F \\
F\end{array}$ & $\begin{array}{l}\mathbf{E} \\
\mathbf{E} \\
\mathbf{E} \\
\mathbf{E} \\
\mathbf{E}\end{array}$ & $\begin{array}{l}\mathbf{E} \\
\mathbf{E} \\
\mathbf{E} \\
\mathbf{E} \\
\mathbf{E}\end{array}$ & $\begin{array}{c}\text { ND } \\
\text { ND } \\
\text { ND } \\
\text { ND } \\
\text { E }\end{array}$ & $\begin{array}{l}\mathbf{F} \\
\mathbf{F} \\
\mathbf{F} \\
\mathbf{E} \\
\mathbf{F}\end{array}$ \\
\hline $\begin{array}{l}P=48 \\
P=47 \\
P=46 \\
P=45 \\
P=44\end{array}$ & $\begin{array}{l}\text { LES }-214-47 \\
\text { LES }-213-47 \\
\text { LES }-212-47 \\
\text { LES }-211-47 \\
\text { LES }-210-47\end{array}$ & $\begin{array}{l}\mathbf{C} \\
\mathbf{C} \\
\mathbf{C} \\
\mathbf{C} \\
\mathbf{C}\end{array}$ & $\begin{array}{l}\mathbf{E} \\
\mathbf{E} \\
\mathbf{E} \\
\text { ND } \\
\text { ND }\end{array}$ & $\begin{array}{l}F \\
F \\
F \\
F \\
F\end{array}$ & $\begin{array}{l}\text { C } \\
\text { C } \\
\text { C } \\
\text { B } \\
\text { C }\end{array}$ & $\begin{array}{l}\mathbf{E} \\
\mathbf{E} \\
\mathbf{E} \\
\mathbf{F} \\
\mathbf{F}\end{array}$ & $\begin{array}{l}\text { ND } \\
\text { ND } \\
\text { ND } \\
\text { ND } \\
\text { ND }\end{array}$ & $\begin{array}{l}\mathbf{G} \\
\mathbf{G} \\
\mathbf{G} \\
\mathbf{G} \\
\mathbf{G}\end{array}$ & $\begin{array}{l}\text { D } \\
\text { C } \\
\text { C } \\
\text { D } \\
\text { D }\end{array}$ & $\begin{array}{l}\text { ND } \\
\text { ND } \\
\text { ND } \\
\text { ND } \\
\text { ND }\end{array}$ & $\begin{array}{l}\text { C } \\
\text { D } \\
\text { D } \\
\text { C } \\
\text { C }\end{array}$ & $\begin{array}{l}\mathbf{E} \\
\mathbf{F} \\
\mathbf{F} \\
\mathbf{E} \\
\mathbf{F}\end{array}$ & $\begin{array}{l}\mathbf{F} \\
\mathbf{F} \\
\mathbf{F} \\
\mathbf{F} \\
\mathbf{F}\end{array}$ & $\begin{array}{l}\mathbf{E} \\
\mathbf{E} \\
\mathbf{E} \\
\mathbf{F} \\
\mathbf{F}\end{array}$ & $\begin{array}{l}\text { B } \\
\text { A } \\
\text { A } \\
\text { C } \\
\text { C }\end{array}$ & $\begin{array}{l}\text { ND } \\
\text { G } \\
\text { G } \\
\text { ND } \\
\text { ND }\end{array}$ & $\begin{array}{l}\mathbf{E} \\
\mathbf{E} \\
\mathbf{D} \\
\mathbf{D} \\
\mathbf{D}\end{array}$ & $\begin{array}{l}\text { ND } \\
F \\
F \\
F \\
\text { ND }\end{array}$ & $\begin{array}{l}\mathbf{E} \\
\mathbf{E} \\
\mathbf{E} \\
\mathbf{E} \\
\mathbf{E}\end{array}$ & $\begin{array}{l}\mathbf{E} \\
\mathbf{E} \\
\mathbf{E} \\
\mathbf{E} \\
\mathbf{E}\end{array}$ & $\begin{array}{c}\text { ND } \\
\text { E } \\
\text { E } \\
\text { ND } \\
\text { ND }\end{array}$ & $\begin{array}{l}\mathbf{F} \\
\mathbf{F} \\
\mathbf{F} \\
\mathbf{F} \\
\mathbf{F}\end{array}$ \\
\hline $\begin{array}{l}P=43 \\
P=42 \\
P=41 \\
P=40 \\
P=39\end{array}$ & $\begin{array}{l}\text { LES }-209-47 \\
\text { LES }-208-47 \\
\text { LES }-207-47 \\
\text { LES }-206-47 \\
\text { LES }-189-47\end{array}$ & $\begin{array}{l}\mathbf{C} \\
\mathbf{C} \\
\mathbf{C} \\
\mathbf{C} \\
\mathbf{C}\end{array}$ & $\begin{array}{l}\mathbf{E} \\
\mathbf{E} \\
\mathbf{E} \\
\mathbf{E} \\
\mathbf{N D}\end{array}$ & $\begin{array}{l}\mathbf{F} \\
\mathbf{F} \\
\mathbf{F} \\
\mathbf{F} \\
\mathbf{F}\end{array}$ & $\begin{array}{l}\text { C } \\
\text { B } \\
\text { A } \\
\text { C } \\
\text { A }\end{array}$ & $\begin{array}{l}\mathbf{E} \\
\mathbf{E} \\
\mathbf{E} \\
\mathbf{E} \\
\mathbf{E}\end{array}$ & $\begin{array}{l}\text { ND } \\
\text { ND } \\
\text { ND } \\
\text { ND } \\
\text { ND }\end{array}$ & $\begin{array}{l}\mathbf{G} \\
\mathbf{G} \\
\mathbf{G} \\
\mathbf{G} \\
\mathbf{G}\end{array}$ & $\begin{array}{l}\text { C } \\
\text { C } \\
\text { C } \\
\text { C } \\
\text { D }\end{array}$ & $\begin{array}{l}\text { ND } \\
\text { ND } \\
\text { ND } \\
\text { ND } \\
\text { ND }\end{array}$ & $\begin{array}{l}\text { D } \\
\text { D } \\
\text { D } \\
\text { D } \\
\text { D }\end{array}$ & $\begin{array}{l}\mathbf{F} \\
\mathbf{F} \\
\mathbf{F} \\
\mathbf{F} \\
\mathbf{F}\end{array}$ & $\begin{array}{l}\mathbf{F} \\
\mathbf{F} \\
\mathbf{F} \\
\mathbf{F} \\
\mathbf{F}\end{array}$ & $\begin{array}{l}\mathbf{E} \\
\mathbf{E} \\
\mathbf{E} \\
\mathbf{E} \\
\mathbf{E}\end{array}$ & $\begin{array}{l}\mathbf{A} \\
\mathbf{C} \\
\mathbf{B} \\
\mathbf{B} \\
\mathbf{C}\end{array}$ & $\begin{array}{l}\mathbf{G} \\
\mathbf{G} \\
\mathbf{G} \\
\mathbf{G} \\
\mathbf{G}\end{array}$ & $\begin{array}{l}\mathbf{E} \\
\mathbf{E} \\
\mathbf{E} \\
\mathbf{E} \\
\mathbf{D}\end{array}$ & $\begin{array}{l}\mathbf{F} \\
\mathbf{F} \\
\mathbf{E} \\
\mathbf{F} \\
\mathbf{E}\end{array}$ & $\begin{array}{l}\mathbf{E} \\
\mathbf{E} \\
\mathbf{E} \\
\mathbf{E} \\
\mathbf{E}\end{array}$ & $\begin{array}{l}\mathbf{E} \\
\mathbf{E} \\
\mathbf{D} \\
\mathbf{E} \\
\mathbf{F}\end{array}$ & $\begin{array}{c}\mathbf{E} \\
\mathbf{E} \\
\mathbf{E} \\
\mathbf{N D} \\
\mathbf{E}\end{array}$ & $\begin{array}{l}\mathbf{F} \\
\mathbf{F} \\
\mathbf{F} \\
\mathbf{F} \\
\mathbf{F}\end{array}$ \\
\hline $\begin{array}{l}P=38 \\
P=37 \\
P=36 \\
P=35 \\
P=34\end{array}$ & $\begin{array}{l}\text { LES -188-47 } \\
\text { LES -1 } 187-47 \\
\text { LES }-186-47 \\
\text { LES - } 185-47 \\
\text { LES -184-47 }\end{array}$ & $\begin{array}{l}\mathbf{C} \\
\mathbf{C} \\
\mathbf{C} \\
\mathbf{C} \\
\mathbf{C}\end{array}$ & $\begin{array}{l}\text { ND } \\
\text { ND } \\
\text { ND } \\
\text { ND } \\
\text { ND }\end{array}$ & $\begin{array}{l}\mathbf{F} \\
\mathbf{F} \\
\mathbf{F} \\
\mathbf{F} \\
\mathbf{F}\end{array}$ & $\begin{array}{l}\text { C } \\
\text { B } \\
\mathbf{C} \\
\mathbf{C} \\
\mathbf{B}\end{array}$ & $\begin{array}{l}\mathbf{D} \\
\mathbf{E} \\
\mathbf{E} \\
\mathbf{E} \\
\mathbf{E}\end{array}$ & $\begin{array}{l}\text { ND } \\
\text { ND } \\
\text { ND } \\
\text { ND } \\
\text { ND }\end{array}$ & $\begin{array}{l}\mathbf{G} \\
\mathbf{G} \\
\mathbf{G} \\
\mathbf{G} \\
\mathbf{G}\end{array}$ & $\begin{array}{l}\mathbf{D} \\
\mathbf{D} \\
\mathbf{D} \\
\mathbf{D} \\
\mathbf{D}\end{array}$ & $\begin{array}{l}\text { ND } \\
\text { ND } \\
\text { ND } \\
\text { ND } \\
\text { ND }\end{array}$ & $\begin{array}{l}\text { D } \\
\text { C } \\
\text { C } \\
\text { D } \\
\text { D }\end{array}$ & $\begin{array}{l}\mathbf{F} \\
\mathbf{E} \\
\mathbf{F} \\
\mathbf{F} \\
\mathbf{F}\end{array}$ & $\begin{array}{l}\mathbf{F} \\
\mathbf{F} \\
\mathbf{F} \\
\mathbf{F} \\
\mathbf{F}\end{array}$ & $\begin{array}{l}\mathbf{E} \\
\mathbf{E} \\
\mathbf{E} \\
\mathbf{E} \\
\mathbf{E}\end{array}$ & $\begin{array}{l}\text { A } \\
\text { C } \\
\text { B } \\
\text { C } \\
\text { B }\end{array}$ & $\begin{array}{l}\mathbf{G} \\
\mathbf{G} \\
\mathbf{G} \\
\mathbf{G} \\
\mathbf{G}\end{array}$ & $\begin{array}{l}\mathbf{D} \\
\mathbf{E} \\
\mathbf{E} \\
\mathbf{E} \\
\mathbf{D}\end{array}$ & $\begin{array}{l}\text { ND } \\
\text { ND } \\
\text { ND } \\
\text { ND } \\
\text { ND }\end{array}$ & $\begin{array}{l}\mathbf{E} \\
\mathbf{E} \\
\mathbf{E} \\
\mathbf{E} \\
\mathbf{E}\end{array}$ & $\begin{array}{l}\mathbf{F} \\
\mathbf{F} \\
\mathbf{F} \\
\mathbf{F}\end{array}$ & $\begin{array}{l}\mathbf{F} \\
\mathbf{E} \\
\mathbf{E} \\
\mathbf{F} \\
\mathbf{E}\end{array}$ & $\begin{array}{l}\mathbf{F} \\
\mathbf{F} \\
\mathbf{F} \\
\mathbf{F} \\
\mathbf{F}\end{array}$ \\
\hline $\begin{array}{l}P=33 \\
P=32 \\
P=31 \\
P=30 \\
P=29\end{array}$ & $\begin{array}{l}\text { LES - } 183-47 \\
\text { LES - } 182-47 \\
\text { LES }-181-47 \\
\text { LES -180-47 } \\
\text { FCA- } 83-47\end{array}$ & $\begin{array}{l}\mathbf{C} \\
\mathbf{C} \\
\mathbf{C} \\
\mathbf{C} \\
\mathbf{C}\end{array}$ & $\begin{array}{l}\text { ND } \\
\text { ND } \\
\text { ND } \\
\mathbf{E} \\
\mathbf{E}\end{array}$ & $\begin{array}{l}\mathbf{F} \\
\mathbf{E} \\
\mathbf{F} \\
\mathbf{F} \\
\mathbf{F}\end{array}$ & $\begin{array}{l}\mathbf{B} \\
\mathbf{A} \\
\mathbf{A} \\
\mathbf{A} \\
\mathbf{B}\end{array}$ & $\begin{array}{l}\mathbf{E} \\
\mathbf{E} \\
\mathbf{E} \\
\mathbf{D} \\
\mathbf{E}\end{array}$ & $\begin{array}{l}\text { ND } \\
\text { ND } \\
\text { ND } \\
\text { ND } \\
\text { ND }\end{array}$ & $\begin{array}{l}\mathbf{G} \\
\mathbf{G} \\
\mathbf{G} \\
\mathbf{G} \\
\mathbf{G}\end{array}$ & $\begin{array}{l}\mathbf{D} \\
\mathbf{C} \\
\mathbf{D} \\
\mathbf{C} \\
\mathbf{C}\end{array}$ & $\begin{array}{l}\text { ND } \\
\text { ND } \\
\text { ND } \\
\text { ND } \\
\text { ND }\end{array}$ & $\begin{array}{l}\mathbf{D} \\
\mathbf{D} \\
\mathbf{D} \\
\mathbf{D} \\
\mathbf{D}\end{array}$ & $\begin{array}{l}\mathbf{F} \\
\mathbf{E} \\
\mathbf{F} \\
\mathbf{F} \\
\mathbf{F}\end{array}$ & $\begin{array}{l}\mathbf{F} \\
\mathbf{F} \\
\mathbf{F} \\
\mathbf{F} \\
\mathbf{F}\end{array}$ & $\begin{array}{l}\mathbf{E} \\
\mathbf{E} \\
\mathbf{E} \\
\mathbf{E} \\
\mathbf{E}\end{array}$ & $\begin{array}{l}\mathbf{B} \\
\mathbf{A} \\
\mathbf{C} \\
\mathbf{B} \\
\mathbf{B}\end{array}$ & $\begin{array}{l}\mathbf{G} \\
\mathbf{G} \\
\mathbf{G} \\
\mathbf{G} \\
\mathbf{G}\end{array}$ & $\begin{array}{l}\mathbf{E} \\
\mathbf{D} \\
\mathbf{E} \\
\mathbf{D} \\
\mathbf{E}\end{array}$ & $\begin{array}{c}\text { ND } \\
\text { ND } \\
\text { ND } \\
\mathbf{E} \\
\mathbf{E}\end{array}$ & $\begin{array}{l}\mathbf{E} \\
\mathbf{D} \\
\mathbf{E} \\
\mathbf{D} \\
\mathbf{E}\end{array}$ & $\begin{array}{l}\mathbf{F} \\
\mathbf{F} \\
\mathbf{F} \\
\mathbf{E} \\
\mathbf{D}\end{array}$ & $\begin{array}{l}\mathbf{E} \\
\mathbf{E} \\
\mathbf{E} \\
\mathbf{E} \\
\mathbf{E}\end{array}$ & $\begin{array}{l}\mathbf{F} \\
\mathbf{F} \\
\mathbf{F} \\
\mathbf{F} \\
\mathbf{F}\end{array}$ \\
\hline$P-28$ & FCA- $82-47$ & D & $\mathbf{E}$ & $\boldsymbol{F}$ & A & $\mathbf{E}$ & ND & G & C & ND & D & E & $\mathbf{F}$ & $\mathbf{E}$ & C & G & $\mathbf{E}$ & $\mathbf{E}$ & $\mathbf{E}$ & D & $\mathbf{E}$ & $\mathbf{F}$ \\
\hline
\end{tabular}




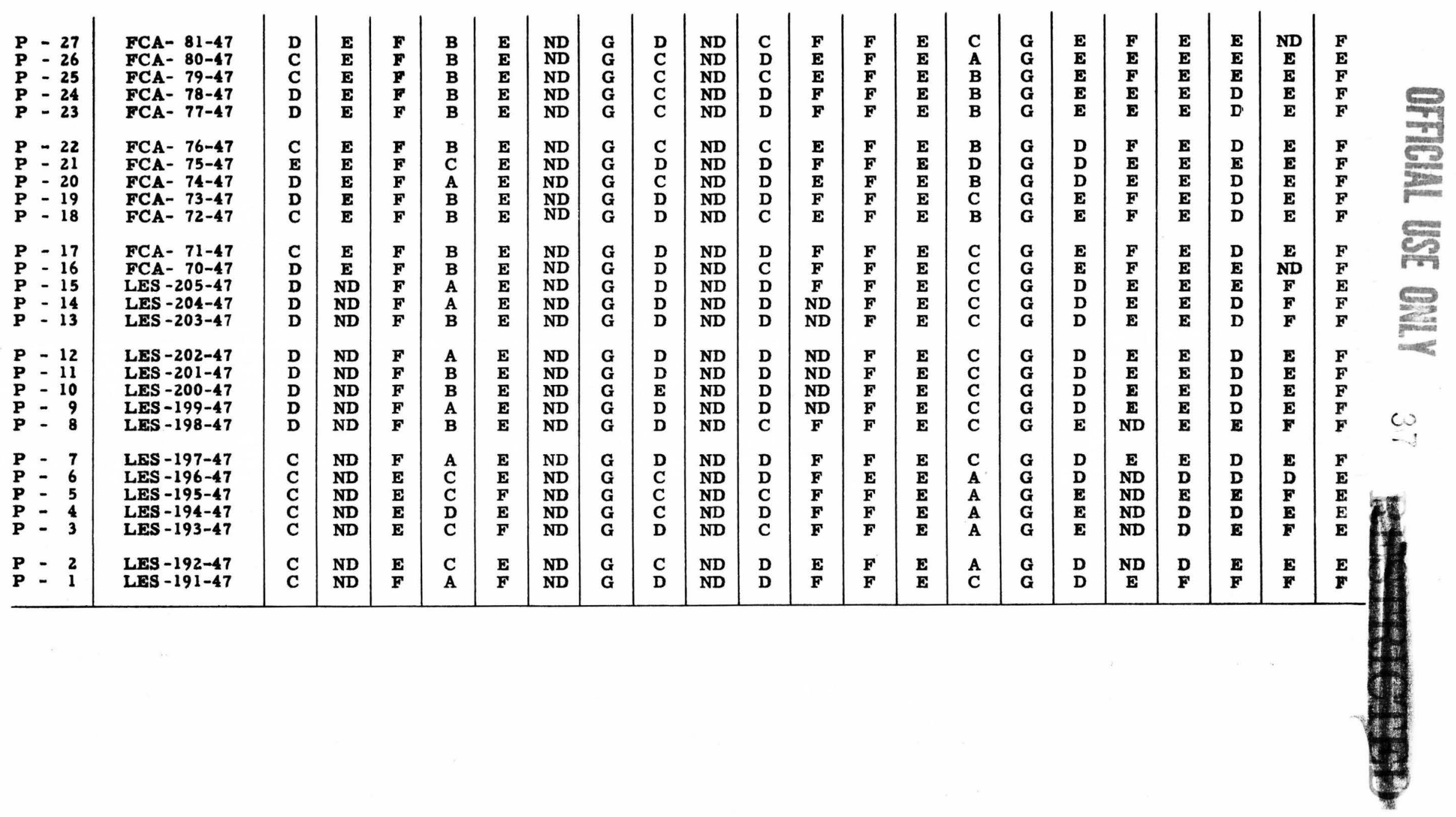


DIAMOND DRILL HOLE 6, SLUG CREEK VALLEY, IDAHO. LOT NO. 1277.

Part of phosphatic shale member of Phosphoria formation cored in diamond drill hole 6 on west slope of hill above Slug Creek Valley, sec. $30, T$. 8 S. R. R4 E., Caribou County, Idaho, on west limb of Schmid syncline. Beds strike north and dip $8^{\circ}$ E. Hole
drilled in Septomber 1948 by U. S. Bureau of Mines. A. E. Long in charge, and core measured and sampled by D. F. Davidson. Samples
analyzed by U. S. Bureau of Mines Laboratory, Albany, Oregon.

\begin{tabular}{|c|c|c|c|c|c|c|c|c|c|}
\hline \multirow{2}{*}{$\begin{array}{l}\text { Bed } \\
\text { no. }\end{array}$} & \multirow{2}{*}{ Rock description } & \multirow{2}{*}{$\begin{array}{l}\text { Sample } \\
\text { no. }\end{array}$} & \multirow{2}{*}{$\begin{array}{c}\text { Thickness } \\
\text { (feet) }\end{array}$} & \multicolumn{2}{|c|}{$\begin{array}{c}\text { Chemical analyses } \\
\text { (percent) }\end{array}$} & \multirow{2}{*}{$\begin{array}{c}\text { Cumulative } \\
\text { thickness } \\
\text { (feet) }\end{array}$} & \multirow{2}{*}{$\begin{array}{l}\text { Thickness } \mathbf{x} \\
\text { percent } \mathbf{P}_{2} \mathrm{O}_{5} \\
\text { (cumulative) }\end{array}$} & \multirow{2}{*}{$\begin{array}{l}\text { Uranium content } \\
\text { (percent) } \\
\text { of (Cbom. U }\end{array}$} & \multirow{2}{*}{$\begin{array}{l}\text { Thickness } x \\
\text { percent eU } \\
\text { (cumulative) }\end{array}$} \\
\hline & & & & $\mathrm{P}_{2} \mathrm{O}_{5}$ & $\begin{array}{c}\text { Acid } \\
\text { insoluble }\end{array}$ & & & & \\
\hline
\end{tabular}

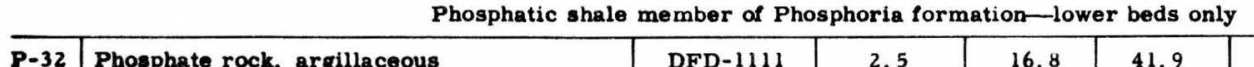

\begin{tabular}{|c|c|}
\hline $\begin{array}{l}P-32 \\
P-31 \\
P-30 \\
P-29 \\
P-28\end{array}$ & $\begin{array}{l}\text { Phosphate rock, argillaceous } \\
\text { Phosphate rock, argillaceous } \\
\text { Phosphate rock, argillaceous } \\
\text { Phouphate rock } \\
\text { Mudstone, phosphatic, calcar }\end{array}$ \\
\hline $\begin{array}{l}P-27 \\
P-26 \\
P-25 \\
P-24 \\
P-23\end{array}$ & $\begin{array}{l}\text { Limestone } \\
\text { Limestone } \\
\text { Phosphate rock, arcillaceous } \\
\text { Limestone } \\
\text { Limostone, argillaceous, phos }\end{array}$ \\
\hline $\begin{array}{l}P-22 \\
P-21 \\
P-20 \\
P-19 \\
P-18\end{array}$ & $\begin{array}{l}\text { Mudstone, phosphatic } \\
\text { Limestone, argillaceous } \\
\text { Limestone, argllaceous } \\
\text { Limestone } \\
\text { Phosphate rock }\end{array}$ \\
\hline $\begin{array}{l}P-17 \\
P-16 \\
P-15 \\
P-14 \\
P-13\end{array}$ & $\begin{array}{l}\text { Phosphate rock } \\
\text { Phosphate rock } \\
\text { Phosphate rock, arclllaceous } \\
\text { Limostone, argilliaceous } \\
\text { Phoephate rock, calcareous }\end{array}$ \\
\hline $\begin{array}{l}\text { P-12 } \\
\text { P-11 } \\
\text { P-10 } \\
\text { P. } 9 \\
\text { P. } 8\end{array}$ & $\begin{array}{l}\text { Phoophate roek } \\
\text { Phomphate rock, argllleceous } \\
\text { Limestone, phoephatic } \\
\text { Limestone, phosphatic } \\
\text { Phoesphate rock }\end{array}$ \\
\hline $\begin{array}{l}\text { P- ? } \\
\text { P. } 6 \\
\text { P. } 5 \\
\text { P. }-3\end{array}$ & $\begin{array}{l}\text { Phoophate rock } \\
\text { Phomphate rock } \\
\text { Phoophate rock } \\
\text { Mudotone } \\
\text { Limostome, aredill }\end{array}$ \\
\hline $\begin{array}{l}\text { P- } 2 \\
\text { P- } 1\end{array}$ & $\begin{array}{l}\text { Mudatone, calcareovin } \\
\text { Phoophati rock }\end{array}$ \\
\hline
\end{tabular}

\begin{tabular}{|c|c|c|c|}
\hline $\begin{array}{l}\text { DFD-1111 } \\
\text { DFD-1 } 1112 \\
\text { DFD-11113 } \\
\text { DFD-11114 } \\
\text { DFD-1115 }\end{array}$ & $\begin{array}{l}2.5 \\
2.0 \\
0.5 \\
2.2 \\
0.7\end{array}$ & $\begin{array}{l}16.8 \\
16.3 \\
21.2 \\
28.0 \\
13.0\end{array}$ & $\begin{array}{l}41.9 \\
39.0 \\
25.4 \\
14.5 \\
29.8\end{array}$ \\
\hline $\begin{array}{l}\text { DFD-1116 } \\
\text { DFD-1117 } \\
\text { DFD-1118 } \\
\text { DFD-1119 } \\
\text { DFD-1120 }\end{array}$ & $\begin{array}{l}2.1 \\
1.4 \\
0.5 \\
0.8 \\
1.3\end{array}$ & $\begin{array}{r}2.4 \\
7.6 \\
22.3 \\
3.7 \\
9.1\end{array}$ & $\begin{array}{r}9.9 \\
17.9 \\
26.0 \\
15.8 \\
27.5\end{array}$ \\
\hline $\begin{array}{l}\text { DFD-1 } 1121 \\
\text { DFD-11122 } \\
\text { DFD-1 } 1123 \\
\text { DFD-1124 } \\
\text { DFD- } 1125\end{array}$ & $\begin{array}{l}1.5 \\
1.6 \\
0.7 \\
0.7 \\
1.3\end{array}$ & $\begin{array}{r}11.3 \\
4.1 \\
0.4 \\
6.7 \\
28.9\end{array}$ & $\begin{array}{l}53.9 \\
28.4 \\
30.8 \\
16.2 \\
14.7\end{array}$ \\
\hline $\begin{array}{l}\text { DFD-1126 } \\
\text { DFD-1127 } \\
\text { DFD-1128 } \\
\text { DFD-1129 } \\
\text { DFD-1130 }\end{array}$ & $\begin{array}{l}1.1 \\
0.8 \\
1.5 \\
1.4 \\
4.2\end{array}$ & $\begin{array}{l}30.9 \\
28.8 \\
22.4 \\
3.6 \\
24.2\end{array}$ & $\begin{array}{l}10.9 \\
14.5 \\
30.2 \\
27.7 \\
11.0\end{array}$ \\
\hline $\begin{array}{l}\text { DFD-1131 } \\
\text { DFD-1132 } \\
\text { DFD-1133 } \\
\text { DFD-1134 } \\
\text { DFD-1135 }\end{array}$ & $\begin{array}{l}1.5 \\
1.1 \\
1.1 \\
0.9 \\
1.0\end{array}$ & $\begin{array}{r}22.8 \\
22.7 \\
13.9 \\
9.8 \\
31.4\end{array}$ & $\begin{array}{r}16.5 \\
23.7 \\
13.4 \\
8.6 \\
7.3\end{array}$ \\
\hline $\begin{array}{l}\text { DFD-1136 } \\
\text { DFD-1137 } \\
\text { DFD-1138 } \\
\text { DFD-11139 } \\
\text { DFD-1 } 140\end{array}$ & $\begin{array}{l}1.3 \\
2.5 \\
2.3 \\
1.0 \\
1.1\end{array}$ & $\begin{array}{r}33.7 \\
33.6 \\
31.8 \\
6.1 \\
2.0\end{array}$ & $\begin{array}{r}2.8 \\
2.3 \\
6.1 \\
64.6 \\
42.6\end{array}$ \\
\hline $\begin{array}{l}\text { DFD-1141 } \\
\text { DFD-1142 }\end{array}$ & $\begin{array}{l}2.1 \\
0.4\end{array}$ & $\begin{array}{r}1.1 \\
30.7\end{array}$ & $\begin{array}{r}36.2 \\
7.4\end{array}$ \\
\hline
\end{tabular}

\begin{tabular}{|l|}
\hline 2.5 \\
4.5 \\
5.0 \\
7.2 \\
7.9 \\
10.0 \\
11.4 \\
11.9 \\
12.7 \\
14.0 \\
15.5 \\
17.1 \\
17.8 \\
18.5 \\
19.8 \\
20.9 \\
21.7 \\
23.2 \\
24.6 \\
28.8 \\
30.3 \\
31.4 \\
32.5 \\
33.4 \\
34.4 \\
35.7 \\
38.2 \\
40.5 \\
41.5 \\
42.6 \\
44.7 \\
45.1
\end{tabular}

\begin{tabular}{|c|c|c|c|}
\hline $\begin{array}{r}42.00 \\
74.60 \\
85.20 \\
116.80 \\
155.90\end{array}$ & $\begin{array}{l}.004 \\
.005 \\
.004 \\
.008 \\
.006\end{array}$ & $\begin{array}{l}-\overline{0} \\
.003 \\
0- \\
.007 \\
.005\end{array}$ & $\begin{array}{l}.010 \\
.020 \\
.022 \\
.040 \\
.044\end{array}$ \\
\hline $\begin{array}{l}160.94 \\
171.58 \\
182.73 \\
185.69 \\
197.52\end{array}$ & $\begin{array}{l}.001 \\
.002 \\
.008 \\
.0005 \\
.003\end{array}$ & $\begin{array}{l}\because- \\
006 \\
-- \\
--\end{array}$ & $\begin{array}{l}.046 \\
.049 \\
.053 \\
.053 \\
.057\end{array}$ \\
\hline $\begin{array}{l}214.47 \\
221.03 \\
221.31 \\
226.00 \\
263.57\end{array}$ & $\begin{array}{l}.006 \\
.002 \\
.001 \\
.002 \\
.006\end{array}$ & \begin{tabular}{c}
.004 \\
\hdashline- \\
\hdashline-0 \\
.006
\end{tabular} & $\begin{array}{l}.066 \\
.069 \\
.070 \\
.071 \\
.079\end{array}$ \\
\hline $\begin{array}{l}297.56 \\
320.60 \\
354.20 \\
359.24 \\
460.88\end{array}$ & $\begin{array}{l}.011 \\
.009 \\
.010 \\
.003 \\
.008\end{array}$ & $\begin{array}{l}.010 \\
.009 \\
.009 \\
.008\end{array}$ & $\begin{array}{l}.091 \\
.098 \\
.113 \\
.118 \\
.151\end{array}$ \\
\hline $\begin{array}{l}495.08 \\
520.05 \\
535.34 \\
544.16 \\
575.56\end{array}$ & $\begin{array}{l}.007 \\
.005 \\
.002 \\
.002 \\
.009\end{array}$ & \begin{tabular}{c}
.006 \\
.004 \\
\hdashline-0 \\
.007
\end{tabular} & $\begin{array}{l}.162 \\
.167 \\
.169 \\
.171 \\
.180\end{array}$ \\
\hline $\begin{array}{l}619.37 \\
703.37 \\
776.51 \\
782.61 \\
784.81\end{array}$ & $\begin{array}{l}.007 \\
.014 \\
.017 \\
.006 \\
.002\end{array}$ & $\begin{array}{l}.007 \\
.013 \\
.017 \\
.004 \\
.-\end{array}$ & $\begin{array}{l}.189 \\
.224 \\
.263 \\
.269 \\
.272\end{array}$ \\
\hline $\begin{array}{l}787.12 \\
799.40\end{array}$ & $\begin{array}{l}.002 \\
.009\end{array}$ & .008 & $\begin{array}{r}.276 \\
.279\end{array}$ \\
\hline
\end{tabular}

Samples analyzed for $\mathrm{eU}$ and chom. U by the S. Geological Survey Laboratory, Geochemistry and Petrology Branch.

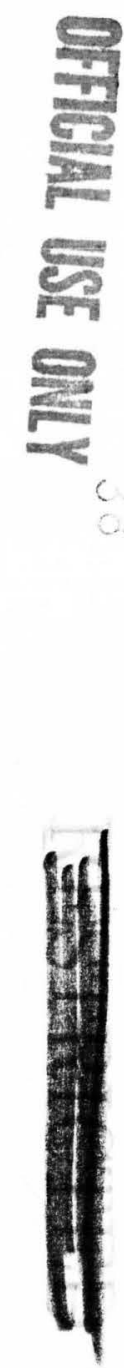




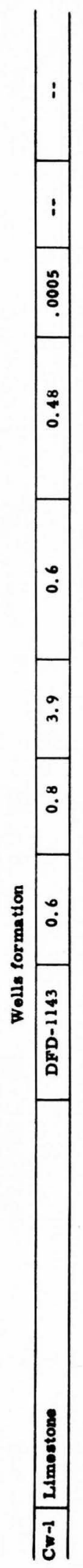


DIAMOND DRILL HOLE 3, MIDDLE SULPHUR CANYON, IDAHO. LOT NO. 1274

Part of phosphatic shale member of Phosphoria formation cored in diamond drill hole 3 on south slope of Middle Sulphur Canyon, sec. 8, T. 9 S., R. 43 E.. Caribou County, Idaho, on west limb of Trail Creek syncline. Beds strike north-northwest and dip $15^{\circ} \mathrm{E}$. Samples analyzed by U. S. Bureau of Mines Laboratory, Albany, Oregon.

Samples analyzed for $\mathrm{eU}$ and chem. U by the U. S. Geological

\begin{tabular}{|c|c|c|c|c|c|c|c|c|c|}
\hline \multirow{2}{*}{$\begin{array}{c}\text { Bed } \\
\text { no. }\end{array}$} & \multirow{2}{*}{ Rock description } & \multirow{2}{*}{$\begin{array}{c}\text { Sample } \\
\text { no. }\end{array}$} & \multirow{2}{*}{$\begin{array}{l}\text { Thickness } \\
\text { (feet) }\end{array}$} & \multicolumn{2}{|c|}{$\begin{array}{c}\text { Chemical analyses } \\
\text { (percent) }\end{array}$} & \multirow{2}{*}{$\begin{array}{l}\text { Cumulative } \\
\text { thickness } \\
\text { (feet) }\end{array}$} & \multirow{2}{*}{$\begin{array}{l}\text { Thickness } x \\
\text { percent } P_{2} O_{5} \\
\text { (cumulative) }\end{array}$} & \multirow{2}{*}{ 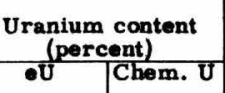 } & \multirow{2}{*}{$\begin{array}{l}\text { Thickness } x \\
\text { percent eU } \\
\text { (cumulative) }\end{array}$} \\
\hline & & & & $\mathrm{P}_{2} \mathrm{O}_{5}$ & $\begin{array}{c}\text { Acld } \\
\text { insoluble }\end{array}$ & & & & \\
\hline
\end{tabular}

Phosphatic shale member of Phosphoria formation-partial section

\begin{tabular}{|c|c|c|c|c|c|c|c|c|c|c|}
\hline $\begin{array}{l}P-25 \\
P-24 \\
P-23 \\
P-22 \\
P-21\end{array}$ & $\begin{array}{l}\text { Mudstone } \\
\text { Mudstone } \\
\text { Mudstone } \\
\text { Mudstone } \\
\text { Mudatone }\end{array}$ & $\begin{array}{l}\text { DFD-961 } \\
\text { DFD }-962 \\
\text { DFD }-963 \\
\text { DFD } 964 \\
\text { DFD }-965\end{array}$ & $\begin{array}{l}1.2 \\
0.8 \\
3.8 \\
2.0 \\
2.3\end{array}$ & $\begin{array}{l}4.4 \\
2.2 \\
3.4 \\
6.4 \\
7.8\end{array}$ & $\begin{array}{l}73.4 \\
84.9 \\
78.9 \\
66.6 \\
65.9\end{array}$ & $\begin{array}{r}1.2 \\
2.0 \\
5.8 \\
7.8 \\
10.1\end{array}$ & $\begin{array}{r}5.28 \\
7.04 \\
19.96 \\
32.76 \\
50.70\end{array}$ & $\begin{array}{l}.003 \\
.002 \\
.003 \\
.003 \\
.002\end{array}$ & $\begin{array}{l}= \\
\because- \\
\because- \\
-\end{array}$ & $\begin{array}{l}.004 \\
.005 \\
.017 \\
.023 \\
.027\end{array}$ \\
\hline $\begin{array}{l}P-20 \\
P-19 \\
P-18 \\
P-17 \\
P-16\end{array}$ & $\begin{array}{l}\text { Mudstone, phosphatic } \\
\text { Mudstone, phosphatic } \\
\text { Phosphate rock, argillaceous } \\
\text { Phosphate rock, argillaceous } \\
\text { Phosphate rock, calcareous }\end{array}$ & $\begin{array}{l}\text { DFD }-966 \\
\text { DFD-967 } \\
\text { DFD-968 } \\
\text { DFD-969 } \\
\text { DFD-970 }\end{array}$ & $\begin{array}{l}4.7 \\
2.2 \\
3.5 \\
1.1 \\
2.1\end{array}$ & $\begin{array}{l}12.8 \\
13.5 \\
19.1 \\
24.4 \\
25.0\end{array}$ & $\begin{array}{l}50.6 \\
50.3 \\
37.7 \\
20.6 \\
13.1\end{array}$ & $\begin{array}{l}14.8 \\
17.0 \\
20.5 \\
21.6 \\
23.7\end{array}$ & $\begin{array}{l}110.86 \\
140.56 \\
207.41 \\
234.25 \\
286.75\end{array}$ & $\begin{array}{l}.004 \\
.004 \\
.004 \\
.004 \\
.004\end{array}$ & $\begin{array}{l}- \\
\because \\
\because\end{array}$ & $\begin{array}{l}.046 \\
.055 \\
.069 \\
.073 \\
.082\end{array}$ \\
\hline $\begin{array}{l}P-15 \\
P-14 \\
P-13 \\
P-12 \\
P-11\end{array}$ & $\begin{array}{l}\text { Phosphate rock } \\
\text { Phosphate rock and mudstone } \\
\text { Phosphate rock and mudstone } \\
\text { Phosphate rock, argillaceous } \\
\text { Mudstone, phosphatic }\end{array}$ & $\begin{array}{l}\text { DFD-971 } \\
\text { DFD-972 } \\
\text { DFD }-973 \\
\text { DFD }-974 \\
\text { DFD }-975\end{array}$ & $\begin{array}{l}0.7 \\
0.4 \\
3.0 \\
0.7 \\
1.9\end{array}$ & $\begin{array}{l}25.3 \\
21.4 \\
24.5 \\
18.8 \\
12.8\end{array}$ & $\begin{array}{l}17.3 \\
28.0 \\
16.6 \\
35.1 \\
52.4\end{array}$ & $\begin{array}{l}24.4 \\
24.8 \\
27.8 \\
28.5 \\
30.4\end{array}$ & $\begin{array}{l}304.46 \\
313.02 \\
386.52 \\
399.67 \\
424.00\end{array}$ & $\begin{array}{l}.004 \\
.006 \\
.011 \\
.009 \\
.006\end{array}$ & $\begin{array}{l}.0 \\
.006 \\
.009 \\
.009 \\
.006\end{array}$ & $\begin{array}{l}.084 \\
.087 \\
.120 \\
.126 \\
.138\end{array}$ \\
\hline $\begin{array}{l}\text { P-10 } \\
P=9 \\
\text { P- } 8 \\
P=7 \\
--\end{array}$ & $\begin{array}{l}\text { Phosphate rock, argillaceous } \\
\text { Phosphate rock } \\
\text { Phosphate rock, argillaceous } \\
\text { Mudstone, phasphatic } \\
\text { Core missing }\end{array}$ & $\begin{array}{c}\text { DFD }-976 \\
\text { DFD }-977 \\
\text { DFD-978 } \\
\text { DFD-979 } \\
--\end{array}$ & $\begin{array}{l}2.6 \\
1.3 \\
1.9 \\
0.9 \\
0.3\end{array}$ & $\begin{array}{l}19.8 \\
27.9 \\
20.8 \\
14.5 \\
--\end{array}$ & $\begin{array}{l}39.3 \\
17.2 \\
36.0 \\
51.0 \\
--.\end{array}$ & $\begin{array}{l}33.0 \\
34.3 \\
36.2 \\
37.1 \\
37.4\end{array}$ & $\begin{array}{l}475.48 \\
511.75 \\
551.27 \\
564.32 \\
--\end{array}$ & $\begin{array}{l}.004 \\
.009 \\
.009 \\
.006 \\
-\end{array}$ & $\begin{array}{l}.0 \\
.009 \\
.009 \\
.004 \\
--\end{array}$ & $\begin{array}{l}.148 \\
.160 \\
.177 \\
.182 \\
.--\end{array}$ \\
\hline $\begin{array}{l}\text { P. } 6 \\
\text { P. } 5 \\
\text { P. } 4 \\
\text { P. } 3 \\
P=2\end{array}$ & $\begin{array}{l}\text { Phosphate rock, argillaceous } \\
\text { Phosphate rock, argillaceous } \\
\text { Phosphate rock, argillaceous } \\
\text { Phosphate rock, argillaceos } \\
\text { Phosphate rock, arglllaceous }\end{array}$ & $\begin{array}{l}\text { DFD-980 } \\
\text { DFD-981 } \\
\text { DFD-982 } \\
\text { DFD-983 } \\
\text { DFD-984 }\end{array}$ & $\begin{array}{l}2.4 \\
2.7 \\
1.3 \\
1.3 \\
0.7\end{array}$ & $\begin{array}{l}27.5 \\
28.8 \\
19.9 \\
23.8 \\
28.8\end{array}$ & $\begin{array}{l}21.2 \\
19.7 \\
36.7 \\
27.5 \\
24.1\end{array}$ & $\begin{array}{l}39.8 \\
42.5 \\
43.8 \\
45.1 \\
45.8\end{array}$ & $\begin{array}{l}66.00 * \\
143.76 \\
169.63 \\
200.57 \\
220.73^{* *} \\
\end{array}$ & $\begin{array}{l}.008 \\
.010 \\
.008 \\
.006 \\
.005\end{array}$ & $\begin{array}{l}.006 \\
.009 \\
.006 \\
.004 \\
.003\end{array}$ & $\begin{array}{l}.019 * \\
.046 \\
.057 \\
.064 \\
.068 * * \\
\end{array}$ \\
\hline$p=1$ & $\begin{array}{l}\text { Core missing } \\
\text { Phosphate rock }\end{array}$ & DFD-985 & $\begin{array}{r}22.4 \\
1.2\end{array}$ & 32. & 9.1 & $\begin{array}{l}68.2 \\
6 \% .4\end{array}$ & $38.88 *$ &.$\overline{007}$ & .006 & $.008 *$ \\
\hline
\end{tabular}

* Cumulative data incomplete due to missing information. Computations start from zero after interruption. 
DIAMOND DRILL HOLE 4, MIDDLE SULPHUR CANYON, IDAHO. LOT NO. 1275.

Phos phatilo ahale member \& Phosphoria formation cored in diamond drill hole 4 on north slope of Middle Sulphur Canyon, sec. 8, T. 9 8.p R. 43 F.. Caribou County, Idaho, on woat limb of Trail Creok syncline. Beds strike north-northwest and dip 22. E. Hole Samples analyad by U. S. Buroau of Minos Laboratory. Albany, Oregon.

\begin{tabular}{|c|c|c|c|c|c|c|c|c|c|c|}
\hline \multirow{2}{*}{$\begin{array}{l}\text { Bod } \\
\text { no. }\end{array}$} & \multirow{2}{*}{ Rock description } & \multirow{2}{*}{$\begin{array}{c}\text { Sample: } \\
\text { no. }\end{array}$} & \multirow{2}{*}{$\begin{array}{c}\text { Thickness } \\
\text { (feot) }\end{array}$} & \multicolumn{2}{|c|}{$\begin{array}{c}\text { Chemical analyses } \\
\text { (percent) }\end{array}$} & \multirow{2}{*}{$\begin{array}{l}\text { Cumulative } \\
\text { thickness } \\
\text { (feet) }\end{array}$} & \multirow{2}{*}{$\begin{array}{l}\text { Thickness } x \\
\text { percent } \mathbf{P}_{2} \mathbf{O}_{8} \\
\text { (cumulatifo) }\end{array}$} & \multicolumn{2}{|c|}{$\begin{array}{l}\text { Uranium ceptent } \\
\text { (poreent) }\end{array}$} & \multirow{2}{*}{$\begin{array}{l}\text { Thickness } x \\
\text { pereent eU } \\
\text { (cumulative) }\end{array}$} \\
\hline & & & & $\mathbf{P}_{2} \mathrm{O}_{3}$ & $\begin{array}{l}\text { Acld } \\
\text { insoluble }\end{array}$ & & & 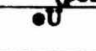 & $c=0$ & \\
\hline \multicolumn{11}{|c|}{ Phosphatic shale momber of Phosphorla formation-basal beds only } \\
\hline $\begin{array}{l}\text { P. } \\
\text { P. } \\
\text { P. } \\
\text { P. } \\
\text { P. } \\
\text { - } \\
\end{array}$ & $\begin{array}{l}\text { Phosphato rock } \\
\text { Phos phate rock } \\
\text { Phoophate rock, argillaceous } \\
\text { Phosphato rock, argillaceous } \\
\text { Phosphate rock }\end{array}$ & $\begin{array}{l}\text { DFD-986 } \\
\text { DFD-987 } \\
\text { DFD-988 } \\
\text { DFD-989 } \\
\text { DFD-990 }\end{array}$ & $\begin{array}{l}1.2 \\
0.9 \\
2.5 \\
4.4 \\
4.1\end{array}$ & $\begin{array}{l}29.1 \\
27.6 \\
27.2 \\
26.6 \\
32.8\end{array}$ & $\begin{array}{r}15.6 \\
19.4 \\
21.8 \\
21.5 \\
5.9\end{array}$ & $\begin{array}{r}1.2 \\
2.1 \\
4.6 \\
9.0 \\
13.1\end{array}$ & $\begin{array}{r}34.92 \\
59.76 \\
127.76 \\
244.80 \\
379.28\end{array}$ & $\begin{array}{l}.008 \\
.010 \\
.008 \\
.006 \\
.009\end{array}$ & $\begin{array}{l}.008 \\
.008 \\
.007 \\
.009 \\
.010\end{array}$ & $\begin{array}{l}.010 \\
.019 \\
.039 \\
.065 \\
.102\end{array}$ \\
\hline $\begin{array}{ll}\text { P. } & 3 \\
\text { P- } & 2 \\
\text { P- } & 1\end{array}$ & $\begin{array}{l}\text { Mudatone } \\
\text { Mudatone } \\
\text { Mudatone, phosphatic }\end{array}$ & $\begin{array}{l}\text { DFD-991 } \\
\text { DFD }-992 \\
\text { DFD-993 }\end{array}$ & $\begin{array}{l}3.0 \\
0.4 \\
0.6\end{array}$ & $\begin{array}{l}2.4 \\
1.2 \\
9.9\end{array}$ & $\begin{array}{l}78.5 \\
80.9 \\
59.7\end{array}$ & $\begin{array}{l}16.1 \\
16.5 \\
17.1\end{array}$ & $\begin{array}{l}386.48 \\
386.96 \\
392.90\end{array}$ & $\begin{array}{l}.004 \\
.003 \\
.005\end{array}$ & $\ddot{000}$ & $\begin{array}{l}.114 \\
.115 \\
.118\end{array}$ \\
\hline \multicolumn{11}{|c|}{ Wells formation } \\
\hline $\mathbf{C w}_{\mathbf{w}-1}$ & Limeatoese & DFD-994 & 1.0 & 0.2 & 2.8 & 1.0 & 0.20 & .0005 & -- & .001 \\
\hline
\end{tabular}

Samplos analyzed for eU and chom. U by the U. 8. Geological Selologt 
DIAMOND DRILL HOLE 5, SOUTH FORK OF JOHNSON CREEK, IDAHO. LOT NO. 1276.

\begin{tabular}{l|l|}
\hline P-7 & Mudstone \\
P-6 & Mudstone, phosphatic \\
P-5 & Mudstone \\
P-4 & Mudstone \\
P-3 & Mudstone \\
P-2 & Mudstone \\
\hline--1 & Core missing \\
P-1 & Mudstone and phosphatic mudstone \\
\hline
\end{tabular}

Part of phosphatic shale member of Phosphoria formation cored in diamond drill hole 5 in valley bottom of south fork of Johnson Creok, sec. 9. T. 9 S., R. 43 E., Caribou County, Idaho, on east limb of Trail Creek syncline. Beds strike northwest and dip $30^{\circ}$ SW. Hole drilled in September 1948 by U. S. Bureau of Mines, A. E. Long in charge, and core measured and sampled by D. F. Davidson. Samples analyzed by U. S. Bureau of Mines Laboratory, Albany, Oregon.

\begin{tabular}{|c|c|c|c|c|c|c|c|c|c|}
\hline \multirow{2}{*}{$\begin{array}{l}\text { Bed } \\
\text { no. }\end{array}$} & \multirow{2}{*}{ Rock description } & \multirow{2}{*}{$\begin{array}{c}\text { Sample } \\
\text { no. }\end{array}$} & \multirow{2}{*}{$\begin{array}{c}\text { Thickness } \\
\text { (feet) }\end{array}$} & \multicolumn{2}{|c|}{$\begin{array}{c}\text { Chemical analyses } \\
\text { (percent) }\end{array}$} & \multirow{2}{*}{$\begin{array}{c}\text { Cumulative } \\
\text { thickness } \\
\text { (feet) }\end{array}$} & \multirow{2}{*}{$\begin{array}{l}\text { Thickness } x \\
\text { percent } \mathrm{P}_{2} \mathrm{O}_{5} \\
\text { (cumulative) }\end{array}$} & \multirow{2}{*}{$\begin{array}{c}\text { Uranium content } \\
\text { (percent) }\end{array}$} & \multirow{2}{*}{$\begin{array}{l}\text { Thickness } x \\
\text { percent eU } \\
\text { (cumulative) }\end{array}$} \\
\hline & & & & $\mathrm{P}_{2} \mathrm{O}_{5}$ & insoluble & & & & \\
\hline
\end{tabular}

Phosphatic shale member of Phosphoria formation-partial section

\begin{tabular}{|l|l|r|r|} 
DFD-995 & 0.5 & 1.8 & 83.0 \\
DFD- 996 & 1.0 & 12.8 & 51.4 \\
DFD- 997 & 1.2 & 2.7 & 76.1 \\
DFD- 998 & 2.3 & 6.6 & 64.0 \\
DFD-1098 & 0.9 & 3.9 & 71.0 \\
DFD-1099 & 2.8 & 6.2 & 67.4 \\
--.9 & 1.1 & --.5 &..- \\
DFD-1:10 & 2.1 & 11.5 & 44.6 \\
\hline
\end{tabular}

\begin{tabular}{r|r}
83.0 & 0.5 \\
51.4 & 1.5 \\
76.1 & 2.7 \\
64.0 & 5.0 \\
71.0 & 5.9 \\
67.4 & 8.7 \\
.-- & 9.8 \\
44.6 & 11.9
\end{tabular}

\begin{tabular}{l|l|l|l|l}
0.5 & 0.90 & .002 & -- & .001 \\
1.5 & 13.70 & .004 & -- & .005 \\
2.7 & 16.94 & .002 & -- & .007 \\
5.0 & 32.12 & .003 & -- & .014 \\
5.9 & 35.63 & .003 & -- & .017 \\
8.7 & $\frac{52.99}{--}$ & .004 & -- & .028 \\
9.8 & --.04 & .006 & .005 & $.013 * *$ \\
1.9 & $24.15 * *$ & .006 &
\end{tabular}

\begin{tabular}{|c|c|c|c|c|}
\hline $\begin{array}{l}0.5 \\
.5 \\
.7 \\
5.9 \\
.9\end{array}$ & $\begin{array}{r}0.90 \\
13.70 \\
16.94 \\
32.12 \\
35.63\end{array}$ & $\begin{array}{l}.002 \\
.004 \\
.002 \\
.003 \\
.003\end{array}$ & $\begin{array}{l}\because- \\
\because- \\
\because- \\
--\end{array}$ & $\begin{array}{l}.001 \\
.005 \\
.007 \\
.014 \\
.017\end{array}$ \\
\hline & $\frac{52.99}{--}$ & .004 & $=$ & .028 \\
\hline 1. & $24.15^{* *}$ & .006 & .005 & $.013 * *$ \\
\hline
\end{tabular}

** Note Incompleteness of cumulative data.

Samples analyzed for $\mathrm{eO}$ and chem. U by the U. S. Geological and Petrolology Branch.
and 
NORTH DAIRY, IDAHO. LOT NO. 1212.

Opper part of phosphatic shale member of Phosphoria formation sampled in bulldozer trench near north end $\alpha$ Dairy syncline, NEtNE \& sec. 20, T. 9 S., R. 44 E., Caribou County, Idaho. The stratigraphic sequence of the units is questionable for, because of a large number of laults, some beds may be omitted or ropealted. No cur R. M. Campbell and L. E.Smith and sampled by R. S. Sears, R. P. Sheldon, and R. A. Smart in August and Seplember 1947. analyzed for $P_{2} O_{F}$ and acid insoluble by U. S. Bureau of Mines Laboratory,

\begin{tabular}{|c|c|c|c|c|c|c|c|c|c|c|}
\hline \multirow{2}{*}{$\begin{array}{l}\text { Bod } \\
\text { no. }\end{array}$} & \multirow[b]{2}{*}{ Rock doscription } & \multirow{2}{*}{$\begin{array}{c}\text { Sample } \\
\text { no. }\end{array}$} & \multirow{2}{*}{$\begin{array}{l}\text { Thickness } \\
\text { (foet) }\end{array}$} & \multicolumn{5}{|c|}{ Chemical analyses (percent) } & \multicolumn{2}{|c|}{ Uranium content (percent) } \\
\hline & & & & $\mathrm{P}_{2} \mathrm{O}_{5}$ & $\mathrm{Al}_{2} \mathrm{O}_{3}$ & $\mathrm{~F}_{2} \mathrm{O}_{3}$ & $\begin{array}{l}\text { Loss on } \\
\text { Ignition }\end{array}$ & $\begin{array}{c}\text { Acld } \\
\text { insoluble }\end{array}$ & $\bullet \mathbf{U}$ & Chem. U \\
\hline \multicolumn{11}{|c|}{ Rex member of Phosphoria formation-basal bed only } \\
\hline R- 1 & Chert & LES- $400-47$ & 0.8 & 6.2 & -- & -- & $-\cdot$ & 79.6 & .003 & .001 \\
\hline
\end{tabular}

\begin{tabular}{|c|c|c|}
\hline & & mber of Phos \\
\hline $\begin{array}{l}\text { P-61 } \\
\text { P-60 } \\
\text { P-59 } \\
\text { P-58 } \\
\text { P-57 }\end{array}$ & $\begin{array}{l}\text { Phosphate rock } \\
\text { Mudstone } \\
\text { Mudstone } \\
\text { Mudstone } \\
\text { Mudstone }\end{array}$ & $\begin{array}{l}\text { LES-399-47 } \\
\text { LES-398-47 } \\
\text { LES-397-47 } \\
\text { LES-396-47 } \\
\text { LES-395-47 }\end{array}$ \\
\hline $\begin{array}{l}P-56 \\
P-55 \\
P-54 \\
P-53 \\
P-52\end{array}$ & $\begin{array}{l}\text { Mudstone } \\
\text { Mudstone } \\
\text { Mudstone } \\
\text { Mudatone } \\
\text { Phosphate rock, arglllaceous }\end{array}$ & $\begin{array}{l}\text { LES-394-47 } \\
\text { LES- } 393-47 \\
\text { LES-392-47 } \\
\text { LES }-391-47 \\
\text { LES }-390-47\end{array}$ \\
\hline $\begin{array}{l}\text { P-51 } \\
\text { P-50 }\end{array}$ & $\begin{array}{l}\text { Mudatione } \\
\text { Mudstone }\end{array}$ & $\begin{array}{l}\text { LES-389-47 } \\
\text { LES- } 388-47\end{array}$ \\
\hline
\end{tabular}

formation-upper part only

\section{Phosphate rock}

P-49 Phosphate rock

P-47
Phosphate rock and mudstone

P-46 Mudatones

P-45 Phosphate rook

P-44 Phosphate rook, eredleceous

P-42 Mudstone, phosphatio

P-41 Phosphate rook \begin{tabular}{l|l} 
P-40 & Phosphate rook, aredlinceous \\
P-39 & Phosphate rook
\end{tabular}

\begin{tabular}{l|l}
0.6 & \\
0.8 & \\
0.5 & \\
2.0 \\
1.2 \\
0.3 \\
1.1 \\
1.5 \\
3.4 \\
1.0 \\
1.3
\end{tabular}

\begin{tabular}{|c|c|}
\hline 30.8 & $\cdots$ \\
\hline 6.9 & $\because$ \\
\hline 1.5 & -- \\
\hline & \\
\hline $\begin{array}{l}3.9 \\
4.7\end{array}$ & 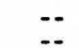 \\
\hline 6.2 & \\
\hline $\begin{array}{r}2.2 \\
26.6\end{array}$ & \\
\hline 3.9 & e \\
\hline 1.0 & \\
\hline-47. & \\
\hline 34.2 & $\begin{array}{l}1.60 \\
7.60\end{array}$ \\
\hline $\begin{array}{l}1.7 \\
27.2\end{array}$ & $\begin{array}{l}7.60 \\
3.4\end{array}$ \\
\hline 5.8 & 11.0 \\
\hline 31.3 & 1.6 \\
\hline 18.8 & 5.0 \\
\hline $\begin{array}{l}28.7 \\
12.5\end{array}$ & $\begin{array}{l}2.1 \\
8.6\end{array}$ \\
\hline 32.7 & 1,1 \\
\hline 24.4 & 3.3 \\
\hline & \\
\hline
\end{tabular}

\begin{tabular}{|c|c|c|}
\hline-- & - & 12.9 \\
\hline 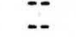 & $\because-$ & 60.1 \\
\hline -. & $\because$ & $\begin{array}{r}79.3 \\
78.6\end{array}$ \\
\hline-- & -- & 67.7 \\
\hline $\bar{~}$ & $\because-$ & $\begin{array}{l}73.9 \\
67.3\end{array}$ \\
\hline-- & - & 78.0 \\
\hline-- & - & 23.3 \\
\hline$\because$ & $\because$ & 69.0 \\
\hline- & - & 83.3 \\
\hline $\begin{array}{l}0.94 \\
3.0 \\
1.61\end{array}$ & $\begin{array}{l}4.26 \\
5.10 \\
5.98\end{array}$ & $\begin{array}{r}7.1 \\
59.2 \\
22.8\end{array}$ \\
\hline $\begin{array}{l}4.3 \\
0.89 \\
1.86 \\
0.92 \\
2.36\end{array}$ & $\begin{array}{l}5.40 \\
4.24 \\
5.92 \\
6.40 \\
6.20\end{array}$ & $\begin{array}{l}69.2 \\
12.8 \\
40.1 \\
16.1 \\
54.5\end{array}$ \\
\hline $\begin{array}{l}1.45 \\
1.29 \\
1.32\end{array}$ & $\begin{array}{l}6.36 \\
7.30 \\
9.92\end{array}$ & $\begin{array}{r}7.3 \\
25.6 \\
18.2\end{array}$ \\
\hline
\end{tabular}

\begin{tabular}{l|c}
.015 & .014 \\
.006 & .001 \\
.007 & .002 \\
.003 & $0 .-$ \\
.002 & -0 \\
.005 & .001 \\
.004 & .001 \\
.004 & .001 \\
.010 & .008 \\
.004 & .001 \\
.002 & .000 \\
& \\
& \\
.013 & .011 \\
.005 & .001 \\
.009 & .010 \\
.004 & .003 \\
.014 & .011 \\
.008 & .009 \\
.016 & .015 \\
.006 & .008 \\
.012 & .016 \\
.011 & .014 \\
.013 & .016
\end{tabular}




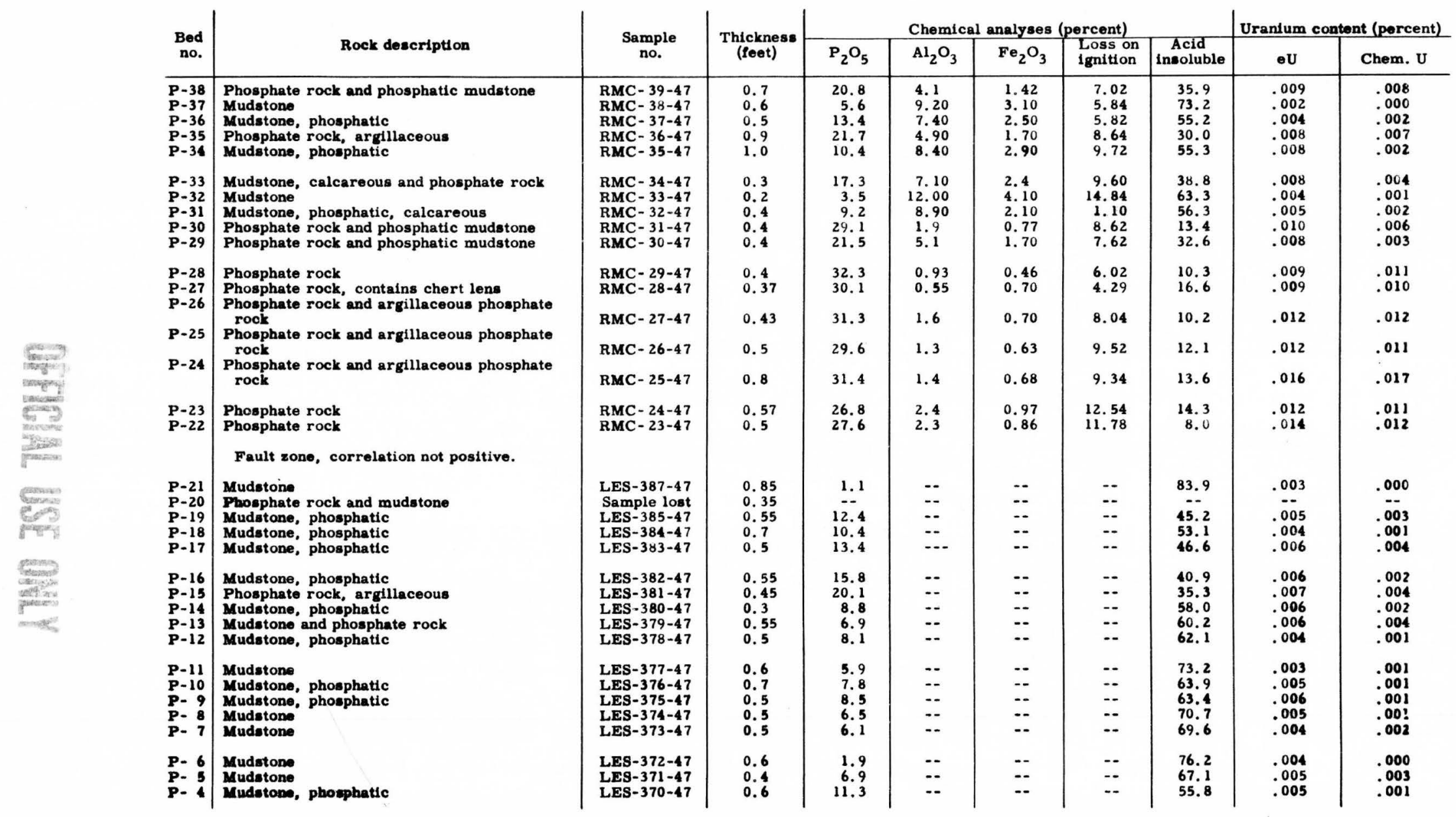




\begin{tabular}{|c|c|c|c|c|c|c|c|c|c|c|}
\hline $\begin{array}{l}\text { P- } 3 \\
\text { P- } 2 \\
\text { P- } 1\end{array}$ & $\begin{array}{l}\text { Mudstone, chert, and phosphate rock } \\
\text { Mudstone, contains cnert nodules } \\
\text { Mudstone, contains chert nodules }\end{array}$ & $\begin{array}{l}\text { Sample lost } \\
\text { LES-368-47 } \\
\text { LES-367-47 }\end{array}$ & $\begin{array}{l}0.3 \\
0.35 \\
2.4\end{array}$ & $\begin{array}{l}-- \\
3.0 \\
2.4\end{array}$ & $\begin{array}{l}-- \\
\because-\end{array}$ & $\begin{array}{l}-- \\
\because- \\
-\end{array}$ & $\begin{array}{l}-- \\
--\end{array}$ & $\begin{array}{l}--. \\
85.3\end{array}$ & $\begin{array}{l}.0 \\
.002 \\
.002\end{array}$ & $\begin{array}{l}\ddot{-} \\
.000\end{array}$ \\
\hline
\end{tabular}


SWAN LAKE GULCH, IDAHO. LOT NO. 1265.

Phosphatic shale member and part of Rex member of Phosphoria formation sampled in bulldozer trench in Swan Lake gulch, $\mathrm{NE} \frac{1}{2} \mathrm{SW} \frac{1}{4}$ sec. 29 , and R. G. Waring and sampled by syncline. Beds of Rex member strike N. 46. gumples analyzed for measured by F. W. O Malley, R. P. Sheld on, Mines Laboratory, Albany, Oregon, and for other constituents by Trace Elements Section Laboratory, U. S. Geological Survey, Washington, D. C.

\begin{tabular}{|c|c|c|c|c|c|c|c|c|c|c|c|c|c|}
\hline \multirow{2}{*}{$\begin{array}{l}\text { Bed } \\
\text { no. }\end{array}$} & \multirow{2}{*}{ Rock description } & \multirow{2}{*}{$\begin{array}{c}\text { Sample } \\
\text { no. }\end{array}$} & \multirow{2}{*}{$\begin{array}{c}\text { Thickness } \\
\text { (feet) }\end{array}$} & \multicolumn{5}{|c|}{ Chemical analyses (percent) } & \multirow{2}{*}{$\begin{array}{l}\text { Cumulative } \\
\text { thickness } \\
\text { (feet) }\end{array}$} & \multirow{2}{*}{$\begin{array}{l}\text { Thickness } x \\
\text { percent } \mathrm{P}_{2} \mathrm{O}_{5} \\
\text { (cumulative) }\end{array}$} & \multicolumn{2}{|c|}{$\begin{array}{c}\text { Uranium content } \\
\text { (percent) }\end{array}$} & \multirow{2}{*}{$\begin{array}{l}\text { Thickness X } \\
\text { percent eU } \\
\text { (cumulative) }\end{array}$} \\
\hline & & & & $\mathrm{P}_{2} \mathrm{O}_{5}$ & $\mathrm{Al}_{2} \mathrm{O}_{3}$ & $\mathrm{Fe}_{2} \mathrm{O}_{3}$ & $\begin{array}{l}\text { Loss on } \\
\text { ignition }\end{array}$ & $\begin{array}{l}\text { Acid } \\
\text { insoluble }\end{array}$ & & & & Chent & \\
\hline \multicolumn{14}{|c|}{ Rex member of Phosphoria formation-basal beds only } \\
\hline $\begin{array}{l}R-8 \\
R-7 \\
R-6 \\
R-5 \\
R-4\end{array}$ & $\begin{array}{l}\text { Chert } \\
\text { Limestone, argillaceous } \\
\text { Chert, calcareous } \\
\text { Chert, calcareous and limestone } \\
\text { Limestone, argillaceous }\end{array}$ & $\begin{array}{l}\text { WOM-3011 } \\
\text { WOM-3020 } \\
\text { WOM-3019 } \\
\text { WOM-3018 } \\
\text { WOM-3017 }\end{array}$ & $\begin{array}{l}2.7 \\
3.5 \\
3.5 \\
2.5 \\
1.5\end{array}$ & $\begin{array}{l}0.2 \\
0.3 \\
0.4 \\
0.6 \\
0.4\end{array}$ & $\begin{array}{l}-- \\
-- \\
-- \\
--\end{array}$ & $\begin{array}{l}-- \\
-- \\
-- \\
--\end{array}$ & $\begin{array}{l}-- \\
-- \\
-- \\
--\end{array}$ & $\begin{array}{l}79.4 \\
35.4 \\
77.4 \\
65.9 \\
24.6\end{array}$ & $\begin{array}{r}2.7 \\
6.2 \\
9.7 \\
12.2 \\
13.7\end{array}$ & $\begin{array}{l}0.54 \\
1.59 \\
2.99 \\
4.49 \\
5.09\end{array}$ & $\begin{array}{l}.0005 \\
.0005 \\
.0005 \\
.0005 \\
.0005\end{array}$ & $\begin{array}{l}-- \\
-- \\
-- \\
--\end{array}$ & $\begin{array}{l}.001 \\
.003 \\
.005 \\
.006 \\
.007\end{array}$ \\
\hline $\begin{array}{l}R-3 \\
R-2 \\
R-1\end{array}$ & $\begin{array}{l}\text { Chert } \\
\text { Chert, calcareous } \\
\text { Chert, calcareous }\end{array}$ & $\begin{array}{l}\text { WOM-3016 } \\
\text { WOM-3015 } \\
\text { WOM-3014 }\end{array}$ & $\begin{array}{l}0.9 \\
5.9 \\
2.9\end{array}$ & $\begin{array}{l}0.5 \\
0.5 \\
0.7\end{array}$ & $\begin{array}{l}=- \\
--\end{array}$ & $\begin{array}{l}-- \\
-- \\
--\end{array}$ & $\begin{array}{l}=- \\
--\end{array}$ & $\begin{array}{l}77.8 \\
70.3 \\
60.7\end{array}$ & $\begin{array}{l}14.6 \\
20.5 \\
23.4\end{array}$ & $\begin{array}{r}5.54 \\
8.49 \\
10.52\end{array}$ & $\begin{array}{l}.0005 \\
.0005 \\
.0005\end{array}$ & $\begin{array}{l}-- \\
--\end{array}$ & $\begin{array}{l}.007 \\
.010 \\
.012\end{array}$ \\
\hline
\end{tabular}

Phosphatic shale member of Phosphoria formation

P-97 Phosphate rock, argillaceous, calcareous: fos. col. no. 48-JES-243

P-96 Mudstone, calcareous

P-95 Mudstone, calcareous 48-JES-241

P-92 Mudstone, calcareous; fos. col. no. 48-JES-240

P-91 Limestone

Mudstone, calcareous; fos. col. no.

P-89 Mudstone, calcareous; fos. col. no.

48-JES-238

P-87 Mudstone, phosphatic

L-86 Limestone, phosphatic

Phestor argilaceous

Phosphate rock, argillaceous, calcareous

P-82 Mudstone, phosphatic; fos. col. no.

WOM-3013
WOM-3012
RGW-3010
RGW-3009
RGW-3008
RGW-3007
RGW-3006
RGW-3005
RGW-3004
RGW-3003
RGW-3002
RGW-3001
RPS- 2850
RPS- 2849
RPS- 2848
WOM-3000

\begin{tabular}{|c|c|c|c|}
\hline 0.9 & 19.5 & -- & -- \\
\hline 1.2 & 1.5 & -- & $\because$ \\
\hline $\begin{array}{l}1.1 \\
2.0\end{array}$ & $\begin{array}{l}6.4 \\
1.8\end{array}$ & $=$ & $=$ \\
\hline 3.5 & 0.6 & -- & -- \\
\hline $\begin{array}{l}1.5 \\
0.6\end{array}$ & $\begin{array}{l}0.9 \\
0.7\end{array}$ & $-\because$ & $=$ \\
\hline 1.0 & 3.5 & -- & -- \\
\hline 2.2 & 3. 2 & -- & -- \\
\hline 0,3 & 123 & 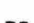 & 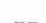 \\
\hline 0.5 & 15.8 & -. & -- \\
\hline 1.0 & 0.8 & -- & -- \\
\hline 1.4 & 32.8 & -- & -- \\
\hline 0.4 & 20.9 & -- & -- \\
\hline 2.7 & 15.8 & -- & -- \\
\hline
\end{tabular}

\begin{tabular}{l|l|} 
& \\
-- & 28.9 \\
-- & 74.8 \\
-- & 54.5 \\
-- & 76.4 \\
& 68.4 \\
-- & 73.9 \\
-- & 19.7 \\
-- & 59.3 \\
-- & 66.1 \\
-- & 72.1 \\
-- & 43.3 \\
-- & 18.9 \\
-- & 47.1 \\
-- & 6.9 \\
& 23.0 \\
-- & 39.4
\end{tabular}

0.9
2.1
3.2
5.2
8.7
10.2
10.8
11.8
14.0
15.7
16.0
16.5
17.5
18.9
19.3
22.0

\begin{tabular}{c|c|c|}
17.55 & .008 & .007 \\
19.35 & .002 & -- \\
26.39 & .005 & .003 \\
29.99 & .002 & -- \\
32.09 & .001 & -- \\
& & \\
33.44 & .002 & -- \\
33.86 & .001 & -- \\
37.36 & .004 & -- \\
44.40 & .003 & -- \\
50.35 & .003 & -- \\
54.04 & .007 & .005 \\
61.94 & .006 & .005 \\
62.74 & .001 & -- \\
108.66 & .010 & .008 \\
117.02 & .012 & .011 \\
& & \\
159.68 & .006 & .005
\end{tabular}




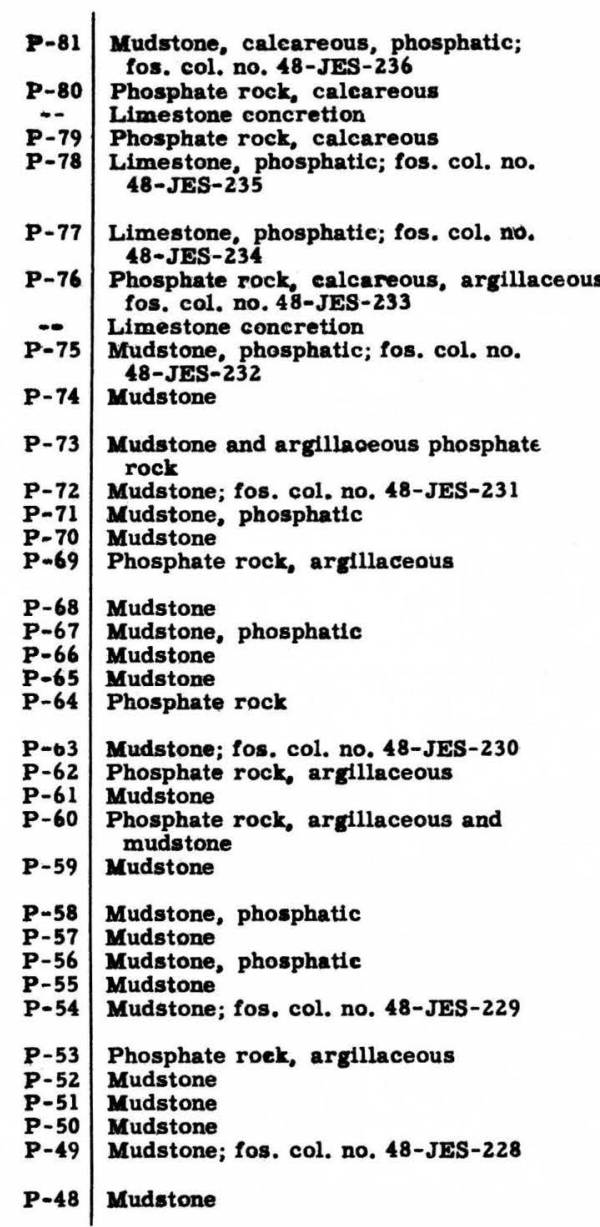

\begin{tabular}{|c|c|c|c|c|c|c|}
\hline $\begin{array}{l}\text { WOM-2999 } \\
\text { WOM-2998 } \\
\text { WOM-2973 } \\
\text { WOM-2997 }\end{array}$ & $\begin{array}{l}2.4 \\
0.8 \\
(0.4) \\
4.0\end{array}$ & $\begin{array}{r}10.3 \\
25.2 \\
0.9 \\
18.2\end{array}$ & $\begin{array}{l}-- \\
\because- \\
--\end{array}$ & $\begin{array}{l}=- \\
=- \\
--\end{array}$ & $\begin{array}{l}-- \\
-- \\
--\end{array}$ & $\begin{array}{r}38.9 \\
13.2 \\
0.7 \\
19.2\end{array}$ \\
\hline WOM-2996 & 1.5 & 12.6 & $=-$ & -- & - & 18.6 \\
\hline WOM-2995 & 1.7 & 15.2 & -- & -- & -- & 18.9 \\
\hline $\begin{array}{l}\text { RPS- } 2847 \\
\text { RPS- } 2846\end{array}$ & $\begin{array}{l}4.2 \\
(0.5)\end{array}$ & $\begin{array}{r}15.8 \\
1.3\end{array}$ & $=$ & $=$ & -- & $\begin{array}{r}25.7 \\
1.5\end{array}$ \\
\hline $\begin{array}{l}\text { RPS- } 2845 \\
\text { RPS- } 2844\end{array}$ & $\begin{array}{l}2.5 \\
0.9\end{array}$ & $\begin{array}{r}11.1 \\
0.9\end{array}$ & -- & $=$ & $=$ & $\begin{array}{l}48.3 \\
85.5\end{array}$ \\
\hline $\begin{array}{l}\text { RPS- } 2843 \\
\text { RPS- } 2842 \\
\text { RPS- } 2841 \\
\text { RPS- } 2840 \\
\text { RPS- } 2839\end{array}$ & $\begin{array}{l}3.3 \\
3.4 \\
0.5 \\
1.3 \\
1.0\end{array}$ & $\begin{array}{r}10.7 \\
5.2 \\
14.4 \\
2.2 \\
18.4\end{array}$ & $\begin{array}{l}=- \\
-- \\
--\end{array}$ & $\begin{array}{l}= \\
-- \\
-- \\
-\end{array}$ & $\begin{array}{l}=- \\
=- \\
=- \\
--\end{array}$ & $\begin{array}{l}52.2 \\
71.6 \\
51.7 \\
85.2 \\
45.2\end{array}$ \\
\hline $\begin{array}{l}\text { RPS- } 2838 \\
\text { WOM-2994 } \\
\text { WOM-2993 } \\
\text { WOM-2992 } \\
\text { WOM-2991 }\end{array}$ & $\begin{array}{l}1.7 \\
4.0 \\
4.8 \\
2.5 \\
0.3\end{array}$ & $\begin{array}{r}1.4 \\
10.2 \\
4.9 \\
5.5 \\
28.3\end{array}$ & $\begin{array}{l}-- \\
-\therefore \\
--\end{array}$ & $\begin{array}{l}=- \\
=- \\
--\end{array}$ & $\begin{array}{l}-- \\
-- \\
--\end{array}$ & $\begin{array}{l}87.2 \\
61.7 \\
75.9 \\
66.6 \\
18.1\end{array}$ \\
\hline $\begin{array}{l}\text { WOM-2990 } \\
\text { WOM-2989 } \\
\text { WOM-2988 }\end{array}$ & $\begin{array}{l}0.8 \\
0.4 \\
1.0\end{array}$ & $\begin{array}{r}5.2 \\
17.4 \\
6.2\end{array}$ & $=$ & $\begin{array}{l}-- \\
--\end{array}$ & 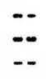 & $\begin{array}{l}71.7 \\
40.9 \\
62.6\end{array}$ \\
\hline $\begin{array}{l}\text { WOM-2987 } \\
\text { WOM-2986 }\end{array}$ & $\begin{array}{l}1.4 \\
1.4\end{array}$ & $\begin{array}{r}15.6 \\
5.9\end{array}$ & $=$ & -- & $=$ & $\begin{array}{l}39.4 \\
63.2\end{array}$ \\
\hline $\begin{array}{l}\text { WOM-2985 } \\
\text { WCM-2984 } \\
\text { WOM-2983 } \\
\text { WOM-2982 } \\
\text { WOM-2981 }\end{array}$ & $\begin{array}{l}1.1 \\
1.8 \\
0.8 \\
2.5 \\
4.0\end{array}$ & $\begin{array}{r}13.6 \\
1.6 \\
14.3 \\
1.1 \\
1.3\end{array}$ & $\begin{array}{l}-- \\
-- \\
--\end{array}$ & $\begin{array}{l}= \\
=- \\
=- \\
--\end{array}$ & $\begin{array}{l}-- \\
=- \\
-\therefore \\
--\end{array}$ & $\begin{array}{l}47.2 \\
85.7 \\
48.4 \\
85.6 \\
85.3\end{array}$ \\
\hline $\begin{array}{l}\text { WOM-2980 } \\
\text { WOM-2979 } \\
\text { WOM-2978 } \\
\text { WOM-2977 } \\
\text { WOM-2976 }\end{array}$ & $\begin{array}{l}0.6 \\
0.6 \\
1.4 \\
0.8 \\
1.6\end{array}$ & $\begin{array}{r}21.6 \\
5.5 \\
3.7 \\
0.3 \\
1.4\end{array}$ & $\begin{array}{l}-- \\
-- \\
-- \\
--\end{array}$ & $\begin{array}{l}-- \\
-- \\
-- \\
--\end{array}$ & $\begin{array}{l}-- \\
-- \\
-- \\
--\end{array}$ & $\begin{array}{l}33.0 \\
71.7 \\
80.8 \\
88.3 \\
83.1\end{array}$ \\
\hline WOM-2975 & 1.0 & 0.4 & -- & -- & - & 84.6 \\
\hline
\end{tabular}

\begin{tabular}{|c|c|c|c|}
\hline $\begin{array}{l}24.4 \\
25.2 \\
--.\end{array}$ & $\begin{array}{r}184.40 \\
204.56\end{array}$ & $\begin{array}{l}.005 \\
.008 \\
.0005\end{array}$ & $\begin{array}{r}.003 \\
.006 \\
-\end{array}$ \\
\hline 29.2 & 277.36 & $.010^{\circ}$ & \\
\hline 30.7 & 296.26 & .007 & .005 \\
\hline 32.4 & 322.10 & .007 & .005 \\
\hline $\begin{array}{c}36.6 \\
-.\end{array}$ & $\begin{array}{r}388.46 \\
--\end{array}$ & $\begin{array}{l}.006 \\
.0005\end{array}$ & .005 \\
\hline $\begin{array}{l}39.1 \\
40.0\end{array}$ & $\begin{array}{l}416.21 \\
417.02\end{array}$ & $\begin{array}{l}.004 \\
.002\end{array}$ & $\because$ \\
\hline $\begin{array}{l}43.3 \\
46.7 \\
47.2 \\
48.5 \\
49.5\end{array}$ & $\begin{array}{l}452.33 \\
470.01 \\
477.21 \\
480.07 \\
498.47\end{array}$ & $\begin{array}{l}.005 \\
.004 \\
.004 \\
.002 \\
.005\end{array}$ & \begin{tabular}{c}
.003 \\
- \\
\hdashline- \\
.004
\end{tabular} \\
\hline $\begin{array}{l}51.2 \\
55.2 \\
60.0 \\
62.5 \\
62.8\end{array}$ & $\begin{array}{l}500.85 \\
541.65 \\
565.17 \\
578.92 \\
587.41\end{array}$ & $\begin{array}{l}.002 \\
.004 \\
.003 \\
.003 \\
.005\end{array}$ & $\begin{array}{l}\because \\
\because \\
-0 \\
.004\end{array}$ \\
\hline $\begin{array}{l}63.6 \\
64.0 \\
65.0\end{array}$ & $\begin{array}{l}591.57 \\
598.53 \\
604.73\end{array}$ & $\begin{array}{l}.002 \\
.004 \\
.003\end{array}$ & $\begin{array}{l}z \\
z\end{array}$ \\
\hline $\begin{array}{l}66.4 \\
67.8\end{array}$ & $\begin{array}{l}626.57 \\
634.83\end{array}$ & $\begin{array}{l}.005 \\
.003\end{array}$ & .003 \\
\hline $\begin{array}{l}68.9 \\
770.7 \\
71.5 \\
74.0 \\
78.0\end{array}$ & $\begin{array}{l}649.79 \\
6552.67 \\
664.11 \\
666.86 \\
672.06\end{array}$ & $\begin{array}{l}.004 \\
.002 \\
.003 \\
.002 \\
.002\end{array}$ & $\begin{array}{l}\because \\
\because\end{array}$ \\
\hline $\begin{array}{l}78.6 \\
79.2 \\
80.6 \\
81.4 \\
83.0\end{array}$ & $\begin{array}{l}685.02 \\
688.32 \\
693.50 \\
693.74 \\
695.98\end{array}$ & $\begin{array}{l}.006 \\
.004 \\
.002 \\
.002 \\
.002\end{array}$ & $\begin{array}{l}.004 \\
\because- \\
\because- \\
--\end{array}$ \\
\hline 84.0 & 696. 38 & .002 & $\cdots$ \\
\hline
\end{tabular}

${ }^{1}$ Fossil collection made by J. E. Smedley, Paleontology and Stratigraphy Branch, U. S. Geological Survey. 


\begin{tabular}{|c|c|c|c|c|c|c|c|c|c|c|c|c|c|c|}
\hline \multirow{2}{*}{$\begin{array}{c}\text { Bed } \\
\text { no. }\end{array}$} & \multirow[b]{2}{*}{ Rock description } & \multirow{2}{*}{$\begin{array}{l}\text { Sample } \\
\text { no. }\end{array}$} & \multirow{2}{*}{$\begin{array}{c}\text { Thickness } \\
\text { (feet) }\end{array}$} & \multicolumn{5}{|c|}{ Chemical analyses (percent) } & \multirow{2}{*}{$\begin{array}{c}\text { Cumulative } \\
\text { thickness } \\
\text { (feet) }\end{array}$} & \multirow{2}{*}{$\begin{array}{c}\text { Thickness } x \\
\text { percent } \mathbf{P}_{2} \mathrm{O}_{5} \\
\text { (cumulative) }\end{array}$} & \multirow{2}{*}{\multicolumn{2}{|c|}{$\begin{array}{c}\text { Uranium content } \\
\text { (percent) }\end{array}$}} & \multirow{2}{*}{$\begin{array}{l}\text { Thickness x } \\
\text { percent eU } \\
\text { (cumulative) }\end{array}$} & \\
\hline & & & & $\mathrm{P}_{2} \mathrm{O}_{5}$ & $\mathrm{Al}_{2} \mathrm{O}_{3}$ & $\mathrm{Fe}_{2} \mathrm{O}_{3}$ & $\begin{array}{l}\text { Loss on } \\
\text { ignition }\end{array}$ & $\begin{array}{c}\text { Acid } \\
\text { insoluble }\end{array}$ & & & & & & \\
\hline $\begin{array}{l}P-47 \\
P-46 \\
P-45 \\
P-44 \\
P-43\end{array}$ & \begin{tabular}{|l} 
Mudstone \\
Phosphate rock, argillaceous \\
Mudstone; fos. col. no. 48-JES-227 \\
Mudstone, phosphatic \\
Mudstone; fos. col. no. 43-JES-226
\end{tabular} & $\begin{array}{l}\text { WOM-2974 } \\
\text { WOM-2972 } \\
\text { WOM-2971 } \\
\text { WOM-2970 } \\
\text { WOM-2969 }\end{array}$ & $\begin{array}{l}1.4 \\
1.7 \\
5.0 \\
1.1 \\
2.0\end{array}$ & $\begin{array}{r}0.4 \\
20.3 \\
1.4 \\
8.1 \\
3.0\end{array}$ & $\begin{array}{l}-- \\
-- \\
-- \\
--\end{array}$ & $\begin{array}{l}= \\
-- \\
\because- \\
--\end{array}$ & $\begin{array}{l}-- \\
-- \\
-- \\
--\end{array}$ & $\begin{array}{l}85.7 \\
34.9 \\
85.5 \\
71.0 \\
76.2\end{array}$ & $\begin{array}{l}85.4 \\
87.1 \\
92.1 \\
93.2 \\
95.2\end{array}$ & $\begin{array}{l}696.94 \\
731.45 \\
738.45 \\
747.36 \\
753.36\end{array}$ & $\begin{array}{l}.002 \\
.005 \\
.002 \\
.003 \\
.003\end{array}$ & $\begin{array}{l}.0 \\
.004 \\
\cdots\end{array}$ & $\begin{array}{l}.342 \\
.351 \\
.361 \\
.364 \\
.370\end{array}$ & \\
\hline $\begin{array}{l}\text { P-42 } \\
\text { P-41 } \\
\text { P-40 } \\
\text { P-39 } \\
\text { P-38 }\end{array}$ & $\begin{array}{l}\text { Phosphate rock, argillaceous } \\
\text { Phosphate rock } \\
\text { Mudstone } \\
\text { Mudstone } \\
\text { Mudstone; fos. col. no. 48-JES-225 }\end{array}$ & $\begin{array}{l}\text { WOM-2968 } \\
\text { WOM-2967 } \\
\text { WOM-2966 } \\
\text { WOM-2965 } \\
\text { WOM-2964 }\end{array}$ & $\begin{array}{l}0.7 \\
0.9 \\
3.5 \\
1.3 \\
3.0\end{array}$ & $\begin{aligned} 18.8 \\
27.7 \\
1.2 \\
1.0 \\
4.2\end{aligned}$ & $\begin{array}{l}=- \\
=- \\
= \\
=\end{array}$ & $\begin{array}{l}-- \\
- \\
-- \\
-\end{array}$ & $\begin{array}{l}-- \\
-- \\
-- \\
--\end{array}$ & $\begin{array}{l}37.3 \\
19.1 \\
88.1 \\
87.1 \\
77.0\end{array}$ & $\begin{array}{r}95.9 \\
96.8 \\
100.3 \\
101.6 \\
104.6\end{array}$ & $\begin{array}{l}766.52 \\
791.45 \\
795.65 \\
796.95 \\
809.55\end{array}$ & $\begin{array}{l}.005 \\
.006 \\
.002 \\
.006 \\
.002\end{array}$ & $\begin{array}{l}.003 \\
.004 \\
.0 \\
.004 \\
-.\end{array}$ & $\begin{array}{l}.374 \\
.379 \\
.386 \\
.394 \\
.400\end{array}$ & mand \\
\hline $\begin{array}{l}P-37 \\
P-36 \\
P-35 \\
P-34 \\
P-33\end{array}$ & $\begin{array}{l}\text { Mudstone } \\
\text { Phosphate rock, argillaceous } \\
\text { Mudstone } \\
\text { Phosphate rock, argillaceous } \\
\text { Mudstone; fos. col. no. 48-JES-224 }\end{array}$ & $\begin{array}{l}\text { WOM-2963 } \\
\text { WOM-2962 } \\
\text { WOM-2961 } \\
\text { WOM-2960 } \\
\text { WOM-2959 }\end{array}$ & $\begin{array}{l}4.4 \\
0.5 \\
4.7 \\
0.5 \\
1.7\end{array}$ & $\begin{array}{r}2.1 \\
22.1 \\
4.2 \\
25.3 \\
1.2\end{array}$ & $\begin{array}{l}-- \\
-- \\
-- \\
-\end{array}$ & $\begin{array}{l}\because- \\
\because- \\
\because-\end{array}$ & $\begin{array}{l}\because \\
\because- \\
\because \\
=\end{array}$ & $\begin{array}{l}87.8 \\
37.8 \\
74.1 \\
24.9 \\
86.5\end{array}$ & $\begin{array}{l}109.0 \\
109.5 \\
114.2 \\
114.7 \\
116.4\end{array}$ & $\begin{array}{l}818.79 \\
829.84 \\
849.58 \\
862.23 \\
864.27\end{array}$ & $\begin{array}{l}.002 \\
.005 \\
.002 \\
.005 \\
.002\end{array}$ & $\begin{array}{l}.0 \\
.003 \\
-0 \\
.003 \\
--\end{array}$ & $\begin{array}{l}.409 \\
.411 \\
.421 \\
.423 \\
.427\end{array}$ & 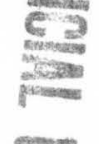 \\
\hline $\begin{array}{l}P-32 \\
P-31 \\
P-30 \\
P-29 \\
P-28\end{array}$ & $\begin{array}{l}\text { Phosphate rock and mudstone } \\
\text { Mudstone; fos. col. no. } 48 \text {-JES-223 } \\
\text { Mudstone, phosphatic, calcareous } \\
\text { Mudstone } \\
\text { Mudstone }\end{array}$ & $\begin{array}{l}\text { WOM-2958 } \\
\text { WOM-2957 } \\
\text { WOM-2956 } \\
\text { WOM-2955 } \\
\text { WOM-2954 }\end{array}$ & $\begin{array}{l}0.7 \\
0.8 \\
2.4 \\
1.8 \\
0.7\end{array}$ & $\begin{array}{r}21.3 \\
2.6 \\
14.5 \\
3.2 \\
3.3\end{array}$ & $\begin{array}{l}-- \\
-- \\
-- \\
-\end{array}$ & $\begin{array}{l}= \\
-- \\
= \\
--\end{array}$ & $\begin{array}{l}-- \\
\ddot{-} \\
= \\
-\end{array}$ & $\begin{array}{l}35.5 \\
82.7 \\
38.7 \\
75.9 \\
75.7\end{array}$ & $\begin{array}{l}117.1 \\
117.9 \\
120.3 \\
122.1 \\
122.8\end{array}$ & $\begin{array}{l}879.18 \\
881.26 \\
916.06 \\
921.82 \\
924.13\end{array}$ & $\begin{array}{l}.005 \\
.002 \\
.004 \\
.002 \\
.003\end{array}$ & $\begin{array}{l}.003 \\
\because- \\
\because- \\
-\end{array}$ & $\begin{array}{l}.430 \\
.432 \\
.441 \\
.445 \\
.447\end{array}$ & trong \\
\hline $\begin{array}{l}P-27 \\
P-26 \\
P-25 \\
P-24 \\
P-23\end{array}$ & $\begin{array}{l}\text { Mudstone } \\
\text { Mudstone } \\
\text { Phosphate rock, argillaceous, calcareous } \\
\text { Mudstone, calcareous } \\
\text { Mudstone, phosphatic, calcareous }\end{array}$ & $\begin{array}{l}\text { WOM-2953 } \\
\text { WOM-2952 } \\
\text { WOM-2951 } \\
\text { WOM-2950 } \\
\text { WOM-2949 }\end{array}$ & $\begin{array}{l}0.8 \\
1.0 \\
0.4 \\
1.1 \\
3.0\end{array}$ & $\begin{array}{r}1.5 \\
4.9 \\
16.4 \\
6.7 \\
13.4\end{array}$ & $\begin{array}{l}-- \\
-- \\
-- \\
--\end{array}$ & $\begin{array}{l}-- \\
-- \\
--\end{array}$ & $\begin{array}{l}-- \\
-- \\
-- \\
--\end{array}$ & $\begin{array}{l}83.3 \\
68.9 \\
31.7 \\
53.7 \\
42.0\end{array}$ & $\begin{array}{l}123.6 \\
124.6 \\
125.0 \\
126.1 \\
129.1\end{array}$ & $\begin{array}{l}925.33 \\
930.23 \\
936.79 \\
944.16 \\
984.36\end{array}$ & $\begin{array}{l}.002 \\
.003 \\
.009 \\
.003 \\
.005\end{array}$ & $\begin{array}{l}-- \\
.008 \\
\overline{0003}\end{array}$ & $\begin{array}{l}.449 \\
.452 \\
.455 \\
.458 \\
.473\end{array}$ & rises \\
\hline $\begin{array}{l}\text { P-22 } \\
P-21 \\
P-20 \\
P-19 \\
P=18\end{array}$ & $\begin{array}{l}\text { Phosphate rock, calcareous, argillaceous } \\
\text { Mudstone, phosphatic } \\
\text { Phosphate rock } \\
\text { Phosphate rock, argillaceous } \\
\text { Limestone }\end{array}$ & $\begin{array}{l}\text { WOM-2948 } \\
\text { WOM-2947 } \\
\text { WOM-2946 } \\
\text { WOM-2945 } \\
\text { WOM-2944 }\end{array}$ & $\begin{array}{l}2.5 \\
0.6 \\
2.6 \\
1.0 \\
2.0\end{array}$ & $\begin{array}{r}21.4 \\
13.3 \\
26.7 \\
17.4 \\
2.8\end{array}$ & $\begin{array}{l}-- \\
-- \\
-- \\
--\end{array}$ & $\begin{array}{l}-- \\
-- \\
-- \\
--\end{array}$ & $\begin{array}{l}-- \\
-- \\
-- \\
--\end{array}$ & $\begin{array}{l}21.3 \\
42.3 \\
14.8 \\
35.2 \\
13.3\end{array}$ & $\begin{array}{l}131.6 \\
132.2 \\
134.8 \\
135.8 \\
137.8\end{array}$ & $\begin{array}{l}1,037.86 \\
1,045.84 \\
1,115.26 \\
1,132.66 \\
1,138.26\end{array}$ & $\begin{array}{l}.004 \\
.005 \\
.012 \\
.010 \\
.001\end{array}$ & $\begin{array}{l}-0 \\
.003 \\
.011 \\
.009 \\
--\end{array}$ & $\begin{array}{l}.483 \\
.486 \\
.518 \\
.528 \\
.530\end{array}$ & \\
\hline $\begin{array}{l}P-17 \\
P-16 \\
P-15 \\
P-14 \\
P-13\end{array}$ & $\begin{array}{l}\text { Mudstone, phosphatic, calcareous } \\
\text { Limestone, argillaceous } \\
\text { Limestone, phosphatic, argillaceous } \\
\text { Phosphate rock, argillaceous } \\
\text { Phosphate rock, argillaceous }\end{array}$ & $\begin{array}{l}\text { WOM-2943 } \\
\text { WOM-2942 } \\
\text { WOM-2941 } \\
\text { WOM-2940 } \\
\text { WOM-2939 }\end{array}$ & $\begin{array}{l}2.3 \\
2.1 \\
0.4 \\
2.9 \\
1.0\end{array}$ & $\begin{array}{r}12.0 \\
1.5 \\
11.7 \\
25.6 \\
19.0\end{array}$ & $\begin{array}{r}\because- \\
\because- \\
2.1 \\
5.1\end{array}$ & $\begin{array}{l}-- \\
-- \\
0.80 \\
1.7\end{array}$ & $\begin{array}{l}= \\
= \\
-- \\
8.74 \\
7.20\end{array}$ & $\begin{array}{l}48.7 \\
21.6 \\
20.0 \\
21.7 \\
37.8\end{array}$ & $\begin{array}{l}140.1 \\
142.2 \\
142.6 \\
145.5 \\
146.5\end{array}$ & $\begin{array}{l}1,165.86 \\
1,169.01 \\
1,173.69 \\
1,247.93 \\
1,266.93\end{array}$ & $\begin{array}{l}.005 \\
.001 \\
.002 \\
.008 \\
.008\end{array}$ & $\begin{array}{l}.003 \\
-- \\
-006 \\
.006\end{array}$ & $\begin{array}{l}.541 \\
.543 \\
.544 \\
.567 \\
.575\end{array}$ & \\
\hline $\begin{array}{l}\text { P-12 } \\
P-11 \\
P-10 \\
P-9 \\
P-8\end{array}$ & $\begin{array}{l}\text { Phosphate rock, argillaceous } \\
\text { Phosphate rock } \\
\text { Phosphate rock, argillaceous } \\
\text { Mudstone; fos, col. no. 48-JES-222 } \\
\text { Phosphate rock, argillaceous; fos. col. } \\
\text { no. 48-JES-221 }\end{array}$ & $\begin{array}{l}\text { WOM-2938 } \\
\text { WOM-2937 } \\
\text { WOM-2936 } \\
\text { WOM-2935 } \\
\text { WOM-2934 }\end{array}$ & $\begin{array}{l}0.7 \\
1.3 \\
1.0 \\
0.3 \\
1.3\end{array}$ & $\begin{array}{r}21.35 \\
27.6 \\
25.6 \\
3.0 \\
22.3\end{array}$ & $\begin{array}{l}2.6 \\
2.7 \\
2.5 \\
8.9 \\
4.0\end{array}$ & $\begin{array}{l}1.3 \\
1.1 \\
0.79 \\
2.8 \\
3.9\end{array}$ & $\begin{array}{l}5.92 \\
6.76 \\
9.02 \\
7.60 \\
6.54\end{array}$ & $\begin{array}{l}34.6 \\
19.7 \\
20.8 \\
77.8\end{array}$ & $\begin{array}{l}147.2 \\
148.5 \\
149.5 \\
149.8 \\
151.1\end{array}$ & $\begin{array}{l}1,281.87 \\
1,317.75 \\
1,343.35 \\
1,344.25 \\
1,373.24\end{array}$ & $\begin{array}{l}.007 \\
.009 \\
.011 \\
.003 \\
.005\end{array}$ & $\begin{array}{c}.006 \\
.007 \\
.009 \\
-0 \\
.004\end{array}$ & $\begin{array}{l}.580 \\
.592 \\
.603 \\
.604 \\
.610\end{array}$ & \\
\hline
\end{tabular}




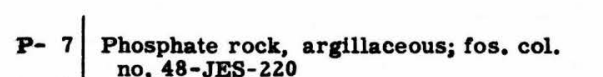

P- 6 Phosphate rock

P- 5 Phosphate rock

P- 4 Phosphate rock; fos. col. no. 48-JES-219

P- 2 Mudston

Careous, phosphatic

P- 1 Phosphate rock

\begin{tabular}{l|} 
WOM-2933 \\
WOM-2932 \\
WOM-2931 \\
WOM-2890 \\
WOM-2889 \\
WOM-2874 \\
WOM-2873
\end{tabular}

\begin{tabular}{l|r|c|c|c|} 
& & & & \\
1.0 & 25.1 & 2.8 & 1.7 & 7.90 \\
1.9 & 33.0 & 1.2 & 0.63 & 7.38 \\
3.6 & 33.7 & 0.81 & 0.48 & 7.92 \\
1.4 & 26.5 & 3.1 & 1.2 & 8.10 \\
1.5 & 8.5 & -- & -- & -- \\
0.9 & 3.9 & -- & -- & -- \\
0.2 & 32.8 & -- & -- & -- \\
\hline
\end{tabular}

\begin{tabular}{r|r|} 
& \\
23.2 & 152.1 \\
5.8 & 154.0 \\
4.7 & 157.6 \\
18.1 & 159.0 \\
53.5 & 160.5 \\
66.7 & 161.4 \\
5.3 & 161.6
\end{tabular}

$1,398.34$
$1,461.04$
$1,582.36$
$1,619.46$
$1,632.21$
$1,635.72$
$1,642.28$

.005
.006
.015
.007
.006
.004
.009

\begin{tabular}{l|}
.004 \\
.005 \\
.0014 \\
.006 \\
.005 \\
.- \\
.008
\end{tabular}

.615

.627

.690

Wells formation

\begin{tabular}{l|l}
3.7 & 1.5 \\
\hline
\end{tabular}

2.40

.000

$--$

.000 
DEER CREEK, IDAHO. LOT NO. 1268.

Phosphatic shale member of Phosphoria formation sampled In Trench I (hand trench) on north side of Deer Creek, Stswt sec. 34. T. 9 8., R. 45 E. . Caribou County, Idaho. Section measured and partially sampled by C. F. Deiss in 1944. Unsampled part of section RPS sertes samples annlyzed by U. S. Bureau of Mines Laboratory, Albany, Oregon.

Samples analyzed for eU and Survey Laboratory, Geochemistry
and Potrology Branch.?

\begin{tabular}{|c|c|}
\hline $\begin{array}{l}\text { Bed } \\
\text { no. }\end{array}$ & Rock description \\
\hline \multicolumn{2}{|r|}{ Phosphat } \\
\hline $\begin{array}{l}\text { P-82 } \\
\text { P-81 } \\
\text { P-80 } \\
\text { P-79 } \\
\text { P-78 }\end{array}$ & $\begin{array}{l}\text { Phouphate rock, calcareous } \\
\text { Mudstone, calcareous } \\
\text { Mudstone, phosphatic } \\
\text { Mudstone, calcareous } \\
\text { Mudatone, calcareous }\end{array}$ \\
\hline $\begin{array}{l}P-77 \\
P-76 \\
P-75 \\
P-74 \\
P-73\end{array}$ & $\begin{array}{l}\text { Mudstone, calcareous } \\
\text { Mudstone, calcareous } \\
\text { Limestone } \\
\text { Mudatone and phosphate rock } \\
\text { Mudatone }\end{array}$ \\
\hline $\begin{array}{l}P-72 \\
P-71\end{array}$ & $\begin{array}{l}\text { Phoesphate rock } \\
\text { Mudetone, calcureous }\end{array}$ \\
\hline & RPS-2827 through RPS-2837 equivalent to \\
\hline $\begin{array}{l}\text { P-70 } \\
\text { P-69 } \\
\text { P-68 }\end{array}$ & $\begin{array}{l}\text { Phoophate rock } \\
\text { Phouphate rock } \\
\text { Mudatono, phosphatic }\end{array}$ \\
\hline $\begin{array}{l}P-67 \\
P-66 \\
P-65 \\
P-64 \\
P-63\end{array}$ & $\begin{array}{l}\text { Limestone } \\
\text { Phosphate rock and mudatono } \\
\text { Phosphate rock } \\
\text { Phosphate rock and mudatone } \\
\text { Phoophate rock }\end{array}$ \\
\hline $\begin{array}{l}P-62 \\
P-61 \\
P-60 \\
P-59 \\
P-58\end{array}$ & $\begin{array}{l}\text { Phosphate rock } \\
\text { Phosphate rook, contains llmestone nodules } \\
\text { Phosphate rock } \\
\text { Phosphate rock, contains llmestone nodules } \\
\text { Limestone, argillaceous }\end{array}$ \\
\hline $\begin{array}{l}P-57 \\
P-56 \\
P-55 \\
P-54 \\
P-53\end{array}$ & $\begin{array}{l}\text { Phosphate rock and mudstane } \\
\text { Mudstone, phosphatic } \\
\text { Phosphate rook } \\
\text { Mudotcne } \\
\text { Phosphate rock, armillacoous }\end{array}$ \\
\hline
\end{tabular}

Sample
no.

\begin{tabular}{|c|c|c|}
\hline \multirow{2}{*}{$\begin{array}{c}\text { Thickness } \\
\text { (feet) }\end{array}$} & \multicolumn{2}{|c|}{$\begin{array}{c}\text { Chemical analyses } \\
\text { (percent) }\end{array}$} \\
\cline { 2 - 3 } & $\mathrm{P}_{2} \mathrm{O}_{5}$ & $\begin{array}{c}\text { Actd } \\
\text { insoluble }\end{array}$ \\
\hline
\end{tabular}

Cumulative
thickness
(feet)

Thickness $x$
percent $P_{2} O_{5}$
(cumulative)

Uranium content

\begin{tabular}{l|l} 
(percent) \\
of
\end{tabular}

\begin{tabular}{c|}
$57-I$ \\
RPS-2837 \\
RPS-2836 \\
RPS-2835 \\
RPS-2834 \\
RPS-2833 \\
RPS-2832 \\
RPS-2831 \\
RPS-2830 \\
RPS-2829 \\
RPS-2828 \\
RPS-2827
\end{tabular}

\begin{tabular}{l|l}
0.3 & \\
0.8 & \\
1.0 & \\
2.0 & \\
6.0 & \\
0.4 & \\
4.5 \\
2.3 \\
0.7 \\
1.8 \\
0.5 \\
3.4
\end{tabular}

\begin{tabular}{r|}
24.1 \\
2.1 \\
5.2 \\
1.6 \\
0.3 \\
0.4 \\
2.3 \\
0.9 \\
11.4 \\
1.9 \\
27.4 \\
1.4
\end{tabular}

\begin{tabular}{l|}
14.9 \\
64.8 \\
69.7 \\
57.1 \\
74.4 \\
67.9 \\
13.4 \\
62.2 \\
78.9 \\
11.2
\end{tabular}

0.3

0.3

(2)

7.23

(2)

unit nos. 69 through 73 .

\begin{tabular}{l|l|r|r|}
$56-1$ & 0.8 & 35.5 & 4.4 \\
$55-1$ & 1.3 & 33.8 & 6.8 \\
$54-1$ & 0.8 & 9.0 & 61.8 \\
--1 & 0.9 &.- &.- \\
$53-1$ & 2.3 & 21.8 & 30.5 \\
$52-1$ & 1.7 & 32.0 & 6.4 \\
$51-1$ & 1.8 & 17.0 & 40.7 \\
$50-1$ & 1.0 & 34.4 & 4.7 \\
$49-1$ & 2.0 & 34.3 & 4.6 \\
$48-1$ & 2.5 & 20.1 & 11.1 \\
$47-1$ & 3.5 & 25.4 & 12.5 \\
$46-1$ & 3.8 & 18.1 & 29.6 \\
$45-1$ & 2.5 & 3.3 & 35.1 \\
$44-1$ & 1.7 & 16.7 & 32.2 \\
$43-1$ & 4.2 & 13.0 & 47.1 \\
$42-1$ & 0.9 & 28.0 & 18.7 \\
$41-1$ & 1.4 & 6.0 & 69.8 \\
$40-1$ & 0.5 & 24.2 & 22.8
\end{tabular}

1.1
2.1
4.1
10.1
10.5
15.0
17.3
18.0
19.8
20.3
23.7

24.5
25.8
26.6
27.5
29.8
31.5
33.3
34.3
36.3
38.8
42.3
46.1
48.6
50.3
54.5
55.4
56.8
57.3

\begin{tabular}{r}
7.23 \\
8.91 \\
14.11 \\
17.31 \\
19.11 \\
19.27 \\
29.62 \\
31.69 \\
39.67 \\
43.09 \\
56.79 \\
61.55 \\
\\
\\
89.95 \\
133.89 \\
134.61 \\
\hline $1 .$. \\
$50.14 *$ \\
104.54 \\
135.14 \\
169.54 \\
238.14 \\
288.39 \\
377.29 \\
446.07 \\
451.82 \\
480.21 \\
534.81 \\
560.01 \\
568.41 \\
580.51
\end{tabular}

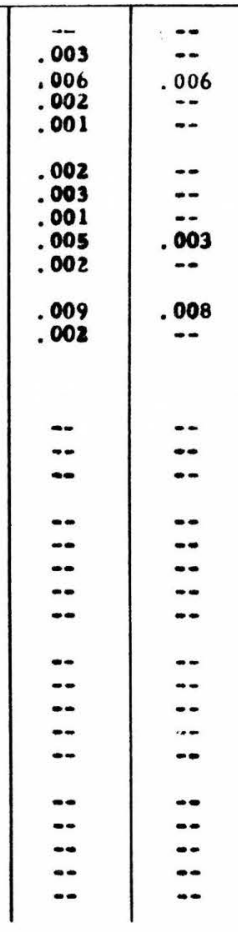




\begin{tabular}{|c|c|c|c|c|c|c|c|c|c|c|}
\hline $\begin{array}{l}\text { P-52 } \\
\text { P-51 }\end{array}$ & \begin{tabular}{|l|} 
Mudetome, phosphatic \\
Limostope
\end{tabular} & RPS-2826 & $\begin{array}{l}2.4 \\
1.0\end{array}$ & $\begin{array}{r}10.7 \\
1.3\end{array}$ & $\begin{array}{l}59.5 \\
12.0\end{array}$ & $\begin{array}{l}59.7 \\
60.7\end{array}$ & $\begin{array}{l}606.19 \\
607.49\end{array}$ & $\ddot{.0005}$ & $\because$ & \\
\hline $\begin{array}{l}P-50 \\
P-49 \\
P-48\end{array}$ & 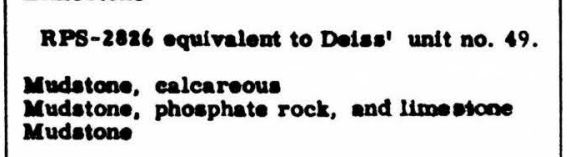 & $\begin{array}{l}38-1 \\
37-1 \\
36-1\end{array}$ & $\begin{array}{l}1.3 \\
1.8 \\
2.2\end{array}$ & $\begin{array}{r}3.2 \\
14.6 \\
2.2\end{array}$ & $\begin{array}{l}59.3 \\
39.9 \\
78.7\end{array}$ & $\begin{array}{l}62.0 \\
63.8 \\
66.0\end{array}$ & $\begin{array}{l}611.65 \\
637.93 \\
642.77\end{array}$ & $\begin{array}{l}\because \\
\because\end{array}$ & $\ddot{z}$ & 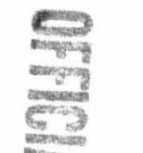 \\
\hline $\begin{array}{l}P-47 \\
P-46 \\
P-45 \\
P-44 \\
P-43\end{array}$ & 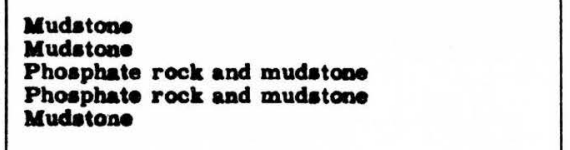 & $\begin{array}{l}35-1 \\
35-1 \\
33-1 \\
332-1 \\
31-1\end{array}$ & $\begin{array}{l}1.5 \\
2.7 \\
1.5 \\
1.2 \\
7.3\end{array}$ & $\begin{array}{r}4.7 \\
2.6 \\
13.4 \\
22.6 \\
2.4\end{array}$ & $\begin{array}{l}64.5 \\
76.3 \\
43.8 \\
25.8 \\
66.5\end{array}$ & $\begin{array}{l}67.5 \\
70.2 \\
71.7 \\
72.9 \\
80.2\end{array}$ & $\begin{array}{l}649.82 \\
656.84 \\
676.94 \\
704.06 \\
721.58\end{array}$ & $\begin{array}{l}\because \\
\because \\
\because \\
\because\end{array}$ & $\begin{array}{l}\because \\
\because \\
\because \\
\because\end{array}$ & $\mathrm{Cos}$ \\
\hline \begin{tabular}{|l|l|}
$P-42$ \\
P-41 \\
$P-40$ \\
$P-39$ \\
$P-38$
\end{tabular} & $\begin{array}{l}\text { Phosphate rock and mudatone } \\
\text { Mudutone and phosphate rock } \\
\text { Limostone and mudetone, phosphatic } \\
\text { Limestone } \\
\text { Mudotone, limestone, and phosphate rock }\end{array}$ & $\begin{array}{l}30-1 \\
29-1 \\
28-1 \\
27-1\end{array}$ & $\begin{array}{l}1.2 \\
0.7 \\
1.4 \\
0.3 \\
3.3\end{array}$ & $\begin{array}{r}17.5 \\
9.6 \\
12.6 \\
9.6\end{array}$ & $\begin{array}{l}42.9 \\
58.2 \\
27.4 \\
39.7\end{array}$ & $\begin{array}{l}81.4 \\
82.1 \\
83.5 \\
83.8 \\
87.1\end{array}$ & $\begin{array}{r}742.58 \\
749.30 \\
766.94 \\
31.68\end{array}$ & $\begin{array}{l}\because \\
\because \\
=\end{array}$ & $\begin{array}{l}\because \\
\because \\
\because\end{array}$ & 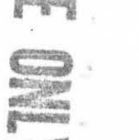 \\
\hline $\begin{array}{l}\text { P-37 } \\
\text { P-36 } \\
\text { P-35 }\end{array}$ & $\begin{array}{l}\text { Mudatone, phosphate rook, and limestone } \\
\text { Muduttone, phosphatic and Ilmestone } \\
\text { Limestone } \\
\text { RPS-2825 equlvalent to Delss' unit no. } 33 .\end{array}$ & $\begin{array}{c}26-1 \\
25-1 \\
\text { RPS-2825 }\end{array}$ & $\begin{array}{l}2.0 \\
3.6 \\
2.7\end{array}$ & $\begin{array}{l}7.9 \\
9.4 \\
0.1\end{array}$ & $\begin{array}{l}30.4 \\
33.6 \\
12.4\end{array}$ & $\begin{array}{l}89.1 \\
92.7 \\
95.4\end{array}$ & $\begin{array}{l}47.48 \\
81.32 \\
81.59\end{array}$ & $\begin{array}{l}\ddot{0} \\
.0005\end{array}$ & $\begin{array}{l}\because \\
\because\end{array}$ & ir \\
\hline $\begin{array}{l}P-34 \\
P-33\end{array}$ & 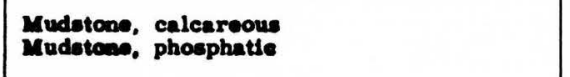 & ${ }_{23-1}^{24-1}$ & $\begin{array}{l}1.8 \\
2.0\end{array}$ & 9.2 & $\begin{array}{l}65.1 \\
53.3\end{array}$ & $\begin{array}{l}97.2 \\
99.2\end{array}$ & $\begin{array}{r}85.19 \\
103.59\end{array}$ & $\because$ & $\because$ & \\
\hline P-32 & 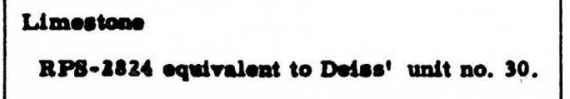 & RPS-2824 & 2.5 & 0.2 & 11.8 & 101.7 & 104.09 & .004 & $\cdots$ & \\
\hline \multirow[t]{2}{*}{$\begin{array}{c}P-31 \\
P-30 \\
P-29 \\
-.-\end{array}$} & 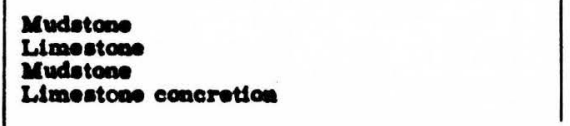 & $\begin{array}{c}22-1 \\
21-1 \\
20-1 \\
20-1 \\
R P S-2823\end{array}$ & $\begin{array}{l}1.2 \\
1.3 \\
1.2 \\
(0.6)\end{array}$ & $\begin{array}{l}2.0 \\
0.3 \\
2.5 \\
0.6\end{array}$ & $\begin{aligned} 76.5 \\
17.2 \\
74.0 \\
3.2\end{aligned}$ & $\begin{array}{l}102.9 \\
107.2 \\
111.4 \\
-.\end{array}$ & $\begin{array}{l}106.49 \\
107.78 \\
118.28 \\
--\end{array}$ & $\begin{array}{l}\ddot{z} \\
\ddot{\operatorname{coos}}\end{array}$ & $\begin{array}{l}\because \\
\because \\
\because\end{array}$ & ety \\
\hline & RPS-2e23 equivalont to Dodes' unit no. 26. & Oncretion oce & a betwree & des- -25 & Aeles- & & & & & 97 \\
\hline$\because$ & $\begin{array}{l}\text { LAmostens coneretion } \\
\text { RPS-2822 coeurs within Deise' unit Do. 25. }\end{array}$ & RPS-2822 & $(1.1)$ & 0.7 & 4.4 & $\cdots$ & -. & .0005 & 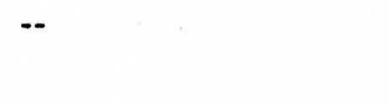 & 4. \\
\hline & 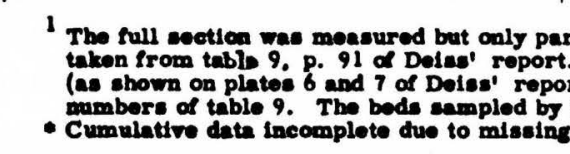 & $\begin{array}{l}\text { of the sects } \\
\text { The beds } n \\
\text { t) and are th } \\
\text { Sheldon (RPS } \\
\text { information. }\end{array}$ & $18 \mathrm{san}$ & Doin & sero: & $\begin{array}{l}\text { lumnar } \\
\text { teorre } \\
\text { ers of } \\
\text { eruptioe }\end{array}$ & $\begin{array}{l}\text { Isted are } \\
\text { Istrench I } \\
\text { be ample }\end{array}$ & \multicolumn{3}{|r|}{ (1) } \\
\hline
\end{tabular}




\begin{tabular}{|c|c|c|c|c|c|c|c|c|c|}
\hline \multirow{2}{*}{$\begin{array}{c}\text { Bed } \\
\text { no. }\end{array}$} & \multirow{2}{*}{ Rock description } & \multirow{2}{*}{$\begin{array}{c}\text { Sample } \\
\text { no. }\end{array}$} & \multirow{2}{*}{$\begin{array}{l}\text { Thickness } \\
\text { (feet) }\end{array}$} & \multicolumn{2}{|c|}{$\begin{array}{c}\text { Chemical analyses } \\
\text { (percent) }\end{array}$} & \multirow{2}{*}{$\begin{array}{l}\text { Cumulative } \\
\text { thickness } \\
\text { (feet) }\end{array}$} & \multirow{2}{*}{$\begin{array}{l}\text { Thickness } x \\
\text { percent } P_{2} \mathbf{O}_{5} \\
\text { (cumulative) }\end{array}$} & \multicolumn{2}{|c|}{$\begin{array}{l}\text { Uranium content } \\
\text { (percent) }\end{array}$} \\
\hline & & & & $\mathbf{P}_{2} \mathrm{O}_{5}$ & $\begin{array}{l}\text { Acid } \\
\text { insoluble }\end{array}$ & & & eù & Chem. U \\
\hline $\begin{array}{l}P-28 \\
P-27 \\
P-26\end{array}$ & $\begin{array}{l}\text { Mudstone } \\
\text { Mudstone, phosphatic and limestone } \\
\text { Limestone } \\
\text { RPS-2821 equivalent to Deiss' unit no. } 23 \text {. }\end{array}$ & $\begin{array}{c}19-1 \\
18-1 \\
R P S-2821\end{array}$ & $\begin{array}{l}5.2 \\
4.5 \\
1.1\end{array}$ & $\begin{array}{l}2.4 \\
7.3 \\
1.7\end{array}$ & $\begin{array}{l}74.0 \\
53.7 \\
18.8\end{array}$ & $\begin{array}{l}116.6 \\
121.1 \\
122.2\end{array}$ & $\begin{array}{l}130.76 \\
163.61 \\
165.48\end{array}$ & $\overline{001}$ & $\begin{array}{l}- \\
\because- \\
--\end{array}$ \\
\hline $\begin{array}{l}P-25 \\
--\end{array}$ & $\begin{array}{l}\text { Phosphate rock, argillaceous and limestone } \\
\text { Limestone concretion? }\end{array}$ & $\begin{array}{c}17-1 \\
\text { RPS-2819 }\end{array}$ & $\begin{array}{l}3.4 \\
(1.2)\end{array}$ & $\begin{array}{r}13.6 \\
1.4\end{array}$ & $\begin{array}{r}29.2 \\
2.3\end{array}$ & 125.6 & $\begin{array}{l}211.72 \\
-. .\end{array}$ & .0005 & $\begin{array}{l}-- \\
--\end{array}$ \\
\hline & RPS-2819 eqtvalent to Deiss' unit no. 21 an & occurs betwe & RPS-2820 & nd Deiss. & & & & & \\
\hline $\begin{array}{l}P-24 \\
P-23 \\
P-22\end{array}$ & $\begin{array}{l}\text { Phosphate rock and calcareous mudstone } \\
\text { Mudstone, phosphatic and limestone } \\
\text { Limestone } \\
\text { RPS-2818 equivalent to Deiss' unit no. } 19 .\end{array}$ & $\begin{array}{l}\text { RPS-2820 } \\
16-1 \\
\text { RPS-2818 }\end{array}$ & $\begin{array}{l}0.5 \\
4.3 \\
1.2\end{array}$ & $\begin{array}{r}15.9 \\
12.0 \\
0.9\end{array}$ & $\begin{array}{r}31.0 \\
37.9 \\
3.0\end{array}$ & $\begin{array}{l}126.1 \\
130.4 \\
131.6\end{array}$ & $\begin{array}{l}219.67 \\
271.27 \\
272.35\end{array}$ & $\overline{--}$ & $\begin{array}{l}.004 \\
-- \\
--\end{array}$ \\
\hline $\begin{array}{l}P-21 \\
P-20\end{array}$ & $\begin{array}{l}\text { Mudstone, phosphatic } \\
\text { Phospnate rock, calcareous, argillaceous }\end{array}$ & $\begin{array}{l}15-1 \\
14-1\end{array}$ & $\begin{array}{l}2.4 \\
1.2\end{array}$ & $\begin{array}{l}10.2 \\
20.4\end{array}$ & $\begin{array}{l}47.0 \\
22.2\end{array}$ & $\begin{array}{l}134.0 \\
135.2\end{array}$ & $\begin{array}{l}296.83 \\
321.31\end{array}$ & $\ddot{-}$ & $\because-$ \\
\hline $\begin{array}{l}\text { P-19 } \\
\text { P-18 } \\
\text { P-17 } \\
\text { P-16 } \\
\text { P-15 }\end{array}$ & $\begin{array}{l}\text { Phosphate rock, calcareous } \\
\text { Phosphate rock, and mudstone } \\
\text { Phosphate rock and mudstone } \\
\text { Limestone, argillaceous } \\
\text { Mudstone, calcareous, phosphatic }\end{array}$ & $\begin{array}{r}13-1 \\
12-1 \\
11-1 \\
10-1 \\
9-1\end{array}$ & $\begin{array}{l}1.5 \\
2.8 \\
1.9 \\
3.0 \\
2.7\end{array}$ & $\begin{array}{r}23.3 \\
23.9 \\
23.9 \\
5.1 \\
9.2\end{array}$ & $\begin{array}{l}14.5 \\
17.0 \\
17.6 \\
20.9 \\
45.8\end{array}$ & $\begin{array}{l}136.7 \\
139.5 \\
141.4 \\
144.4 \\
147.1\end{array}$ & $\begin{array}{l}356.26 \\
423.18 \\
468.59 \\
483.89 \\
508.73\end{array}$ & $\begin{array}{l}\because- \\
\because \\
\because \\
-\end{array}$ & $\begin{array}{l}\because- \\
\because \\
\because- \\
\because\end{array}$ \\
\hline P-14 & $\begin{array}{l}\text { Limestone, argillaceous } \\
\text { RPS-2812 equivalent to Deiss' unit no. } 11 \text {. }\end{array}$ & RPS-281z & 2.5 & 0.6 & 20.9 & 149.6 & 510.23 & -- & -- \\
\hline $\begin{array}{l}P-13 \\
P-12 \\
P-11 \\
--\end{array}$ & $\begin{array}{l}\text { Phosphate rock and mudstone } \\
\text { Phosphate rock, mudstone, and limestone } \\
\text { Phiosphate rock and mudstone } \\
\text { Limestone concretion } \\
\text { RPS-2811 concretion occurs within RPS-28 }\end{array}$ & $\begin{array}{c}8-1 \\
7-1 \\
6-1 \\
\text { RPS-2811 }\end{array}$ & $\begin{array}{l}2.7 \\
3.5 \\
7.6 \\
(0.6)\end{array}$ & $\begin{array}{r}25.2 \\
18.7 \\
25.4 \\
9.8\end{array}$ & $\begin{array}{l}18.4 \\
34.9 \\
17.6 \\
12.8\end{array}$ & $\begin{array}{l}152.3 \\
155.8 \\
163.4 \\
---\end{array}$ & $\begin{array}{l}578.27 \\
643.72 \\
836.76 \\
--\end{array}$ & $\begin{array}{c}\because \\
\because- \\
.003\end{array}$ & $\begin{array}{l}\because- \\
\because \\
\because\end{array}$ \\
\hline $\begin{array}{l}P-10 \\
P-9\end{array}$ & $\begin{array}{l}\text { Phosphate rock and calcareous mudstone } \\
\text { Limestone } \\
\text { RPS-2809 and RPS-2810 equivalent to Deiss }\end{array}$ & $\begin{array}{l}\text { RPS-2810 } \\
\text { RPS-2809 } \\
\text { unit no. } 7 .\end{array}$ & $\begin{array}{l}1.7 \\
1.1\end{array}$ & $\begin{array}{r}24.9 \\
2.3\end{array}$ & $\begin{array}{r}15.7 \\
6.5\end{array}$ & $\begin{array}{l}165.1 \\
166.2\end{array}$ & $\begin{array}{l}879.09 \\
904.39\end{array}$ & .007 & .006 \\
\hline $\begin{array}{l}\text { P- } 8 \\
\text { P- } 7\end{array}$ & $\begin{array}{l}\text { Phosphate rock, argillaceous } \\
\text { Limestone } \\
\text { RPS-2808 equivalent to Deiss' unit no. } 5 \text {. }\end{array}$ & $\begin{array}{c}\text { 5-1 } \\
\text { RPS-2808 }\end{array}$ & $\begin{array}{l}3.0 \\
1.6\end{array}$ & $\begin{array}{r}21.9 \\
6.6\end{array}$ & $\begin{array}{r}34.6 \\
6.9\end{array}$ & $\begin{array}{l}169.2 \\
170.8\end{array}$ & $\begin{array}{l}970.09 \\
980.65\end{array}$ & .001 & $=$ \\
\hline P- 6 & Phosphate rock & 4-I & 0.8 & 28.0 & 8.1 & 171.6 & $1,003.05$ & -- & -- \\
\hline
\end{tabular}




\begin{tabular}{|c|c|c|c|c|c|c|c|c|c|}
\hline $\begin{array}{ll}\text { P- } & 5 \\
\text { P. } & 4 \\
\text { P. } & 3 \\
\text { P- } & 2 \\
\text { P. } & 1\end{array}$ & $\begin{array}{l}\text { Phoophate rock } \\
\text { Phouphate rock } \\
\text { Mudstone } \\
\text { Limestone, argillaceous } \\
\text { Phosphate rock }\end{array}$ & $\begin{array}{c}3-I \\
2-I \\
1-I \\
\text { RPS-2807 } \\
\text { RPS-2806 }\end{array}$ & $\begin{array}{l}3.2 \\
1.5 \\
0.9 \\
3.5 \\
0.5\end{array}$ & $\begin{array}{r}32.1 \\
33.0 \\
2.9 \\
0.3 \\
31.2\end{array}$ & $\begin{array}{r}4.1 \\
3.9 \\
71.8 \\
38.8 \\
6.8\end{array}$ & $\begin{array}{l}174.8 \\
176.3 \\
177.2 \\
180.7 \\
181.2\end{array}$ & $\begin{array}{l}1,105.77 \\
1,155.27 \\
1,157.88 \\
1,158.93 \\
1,174.53 * *\end{array}$ & $\begin{array}{l}-- \\
\because- \\
001 \\
.011\end{array}$ & $\begin{array}{l}\ddot{-} \\
\ddot{0} \\
.010\end{array}$ \\
\hline \multicolumn{10}{|c|}{ Wells formation } \\
\hline $\begin{array}{l}C w-5 \\
C w-4 \\
C w-3 \\
C w-2 \\
C w-1\end{array}$ & $\begin{array}{l}\text { Limestone } \\
\text { Phosphate rock } \\
\text { Limestone } \\
\text { Phosphate rock } \\
\text { Limestone }\end{array}$ & $\begin{array}{l}\text { RPS-2817 } \\
\text { RPS }-2816 \\
\text { RPS }-2815 \\
\text { RPS }-2814 \\
\text { RPS }-2813\end{array}$ & $\begin{array}{l}7.0 \\
0.1 \\
3.0 \\
0.1 \\
1.8\end{array}$ & $\begin{array}{r}1.2 \\
33.4 \\
1.9 \\
34.6 \\
0.7\end{array}$ & $\begin{array}{l}5.6 \\
4.2 \\
3.8 \\
5.9 \\
6.1\end{array}$ & $\begin{array}{r}7.0 \\
7.1 \\
10.1 \\
10.2 \\
12.0\end{array}$ & $\begin{array}{r}8.40 \\
11.74 \\
17.14 \\
20.90 \\
22.16\end{array}$ & $\begin{array}{l}.0005 \\
.013 \\
.0005 \\
.011 \\
.0005\end{array}$ & $\begin{array}{l}\ddot{011} \\
\ddot{009} \\
.0\end{array}$ \\
\hline
\end{tabular}

